\title{
AN IN-DEPTH EXAMINATION OF INTOLERANCE OF UNCERTAINTY AND ITS MODIFICATION IN GENERALIZED ANXIETY DISORDER \\ by
}

Katie Fracalanza

Master of Arts, Ryerson University, 2010

Honours Bachelor of Commerce, McMaster University, 2007

\author{
A dissertation \\ presented to Ryerson University \\ in partial fulfillment of the \\ requirements for the degree of \\ Doctor of Philosophy \\ in the Program of \\ Psychology \\ Toronto, Ontario, Canada, 2015 \\ (C) Katie Fracalanza 2015
}


Author's Declaration for Electronic Submission of a Dissertation

I hereby declare that I am the sole author of this dissertation. This is a true copy of the dissertation, including any required final revisions, as accepted by my examiners.

I authorize Ryerson University to lend this dissertation to other institutions or individuals for the purpose of scholarly research.

I further authorize Ryerson University to reproduce this dissertation by photocopying or by other means, in total or in part, at the request of other institutions or individuals for the purpose of scholarly research.

I understand that my dissertation may be made electronically available to the public. 


\begin{abstract}
An In-Depth Examination of Intolerance of Uncertainty and Its Modification in

Generalized Anxiety Disorder

Doctor of Philosophy, 2015

Katie Fracalanza

Psychology, Ryerson University
\end{abstract}

Two decades of research support Dugas and colleagues’ (1998) Intolerance of Uncertainty (IU) Model of Generalized Anxiety Disorder (GAD), suggesting that IU is a key factor involved in the maintenance of excessive worry. As such, a cognitive behavioural treatment targeting IU (CBT-IU) has been developed, and it is a highly efficacious treatment for GAD. Given the importance of IU in GAD, the current research investigated attitudes and behaviours associated with uncertainty that are not yet well understood, and tested the effects of a technique employed in CBT-IU called uncertainty exposure on IU and GAD symptoms. Study $1 \mathrm{a}$ and $1 \mathrm{~b}$ present the results of a mixed methods study that involved asking individuals with GAD $(n=20)$ and non-psychiatric control participants (NPCs; $n=20$ ) about their experience with uncertainty, coding their responses into themes, and examining between-group differences in responses. Study 1a compared the responses of people with GAD to NPCs on: beliefs about uncertainty, attitudes toward different "types” of uncertainty, and reflections on clinical observations about IU. Study 1b compared the responses of the GAD and NPC groups on: what behaviours they engage in when uncertain, as well as the frequency, functions, discontinuation factors, and problems associated with such behaviours. Study 1a and 1b produced rich data that offer novel insights about the nature of IU in GAD. Study 2 tested the impact of completing a single session of training in uncertainty exposure and 1 week of practice with uncertainty exposure (exposure group; $n=20$ ), compared to completing assessment only (control 
group; $n=20$ ) in a GAD sample. Participants in the exposure group showed large significant improvements in IU and GAD symptoms from baseline to 1 or 2 weeks post baseline, whereas the control group showed no change. Completing more uncertainty exposure practice was associated with larger improvement in outcomes. These findings provide novel support for the proposed role of IU in maintaining GAD, and for the use of uncertainty exposure in GAD treatment. Overall, findings from this dissertation inform theoretical conceptualizations of IU in GAD and the clinical application of this model. 


\section{Acknowledgements}

To my mentor - Dr. Naomi Koerner - Thank you for teaching me, inspiring me, and supporting me, especially during times when the learning curve was steep. Your wealth of knowledge has nourished my love of science. I deeply appreciate all that you have done for me and my career.

To my committee members - Dr. Martin Antony and Dr. Kelly McShane - your respective expertise and contributions have made this project possible. To Dr. Antony, I am grateful for your invaluable feedback and guidance, not only over the course of this project, but throughout my graduate school journey. To Dr. McShane, thank you for making time for many consultations and questions about this work, even throughout your maternity leave. I would also like to thank the additional members of my examining committee, Dr. Nancy Walton and Dr. David Dozois, for making time to review my dissertation and participate in my defense.

To my labmates - I've thoroughly enjoyed the companionship and laughs we've shared inside and outside the lab. You brightened my days and made our workspace fun. I appreciate the help and encouragement you each provided whenever I needed it.

To my Ryerson cohort - as we've joked about many times, the bond we share cannot be captured in an acknowledgements section. I am grateful for the incredible memories and lifelong friendships we've built.

To my family and my partner - I can't imagine completing this dissertation without your constant love and support. Thank you so much for being there, believing in me, and accepting me unconditionally; I am extremely blessed to have you.

Finally, I would like to acknowledge the external funding sources for this project - the Canadian Institutes of Health Research and the Ontario Graduate Scholarship Program. 


\section{Contents}

General Introduction..................................................................... 1

Study 1a: Exploring Attitudes toward Uncertainty in People with GAD and Non-

Psychiatric Control Participants............................................. 9

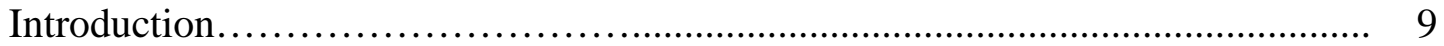

Present Study .......................................................................... 13

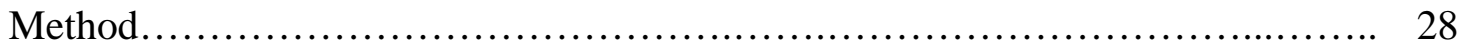

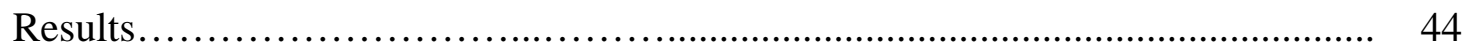

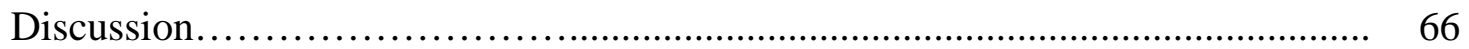

Study 1b: Behavioural Responses to Uncertainty in People with GAD and Non- 101

Psychiatric Control Participants............................................... 101

Introduction.......................................................................... 101

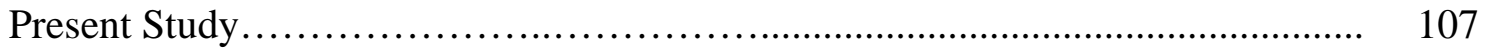

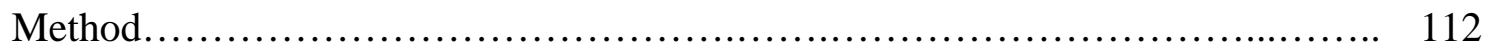

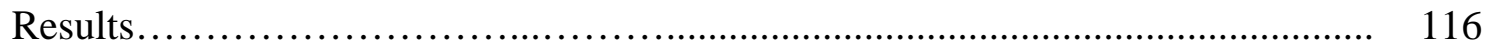

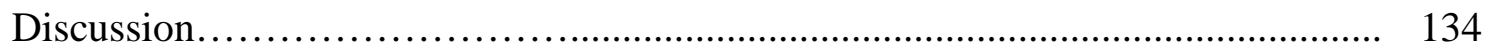

Study 2: The Effects of Uncertainty Exposure on GAD-Relevant Symptoms and

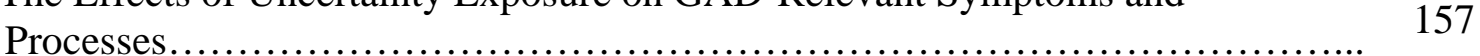

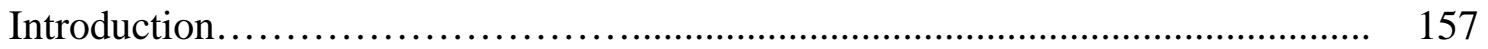

Present Study ...................................................................... 162

Method................................................................... 165

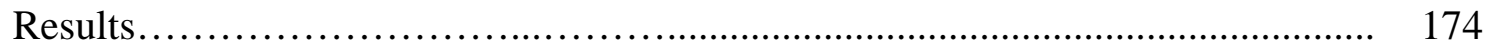

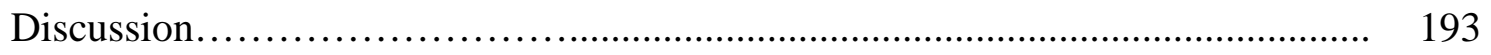

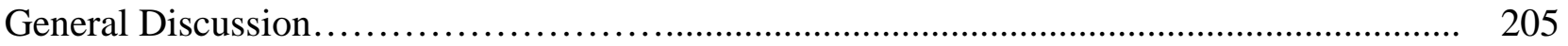

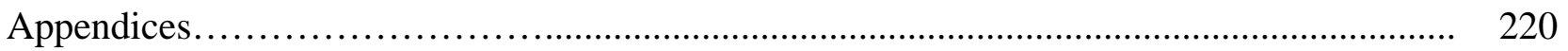

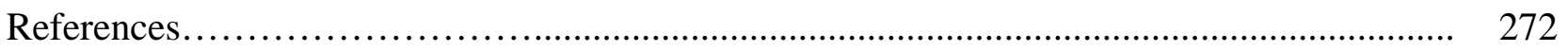


Table 1: Demographic Characteristics by Group................................. 30

Table 2: Clinical Characteristics by Group.................................... 32

Table 3: Interrater Reliability by Interview Question............................... 42

Table 4: Underlying Thoughts, Beliefs, and Assumptions about Uncertainty................... 45

Table 5: Factors That May Moderate Attitudes toward Potential Negative Outcomes..... 54

Table 6: Exploring Uncertainty As Positive or Neutral................................ 58

Table 7: Systematically Investigating Clinical Observations about Intolerance of

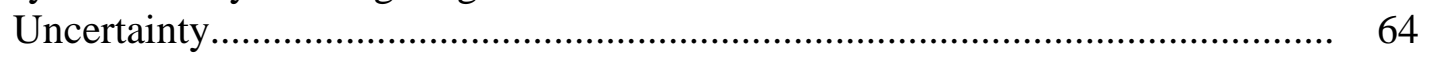

Table 8: Typical Behavioural Responses to an Uncertain Situation.................... 117

Table 9: Frequency of Behaviours Participants Used When Uncertain over the Past Month......................................................... 120

Table 10: Functions of Behavioural Responses to Uncertainty........................ 125

Table 11: Cessation Factors of Behavioural Responses to Uncertainty................. 128

Table 12: Impairment Associated with Behavioural Responses to Uncertainty........... 133

Table 13: Study 2 - Demographic Characteristics by Condition..................... 167

Table 14: Types of Uncertainty Exposures that Participants with GAD Completed.......... 175

Table 15: Scores on GAD-Related Symptoms and Cognitions by Condition.............. 178 
Table 16: Summary of Zero-Order Correlations between Change in IU-Related Cognitions and GAD Symptoms in the Exposure and Control Conditions........

Table 17: Multiple Regressions Predicting Weekly GAD Symptoms at 1- and 2-Week Post Baselines from Improvement on IU-Related Cognitions..................

Table 18: Multilevel Models for Daily Outcomes as Associated with Time and Condition.

Table 19: Zero-Order Correlations of Homework Compliance and Anxiety Change Expectancy to Change Scores on GAD-Related Outcomes from Baseline to 1-Week Post Baseline in the Exposure Condition............................

Table 20: Zero-Order Correlations of Homework Compliance and Anxiety Change Expectancy to Change Scores on GAD-Related Outcomes from Baseline to 2 Weeks Post Baseline in the Exposure Condition............................

Table 21: Multilevel Models for Daily Outcomes as Associated with Time and Homework-Related Variables in the Exposure Condition............................. 


\section{List of Appendices}

Appendix A: Participant Recruitment Materials............................... 220

Appendix B: Qualitative Interview Guide....................................................... 222

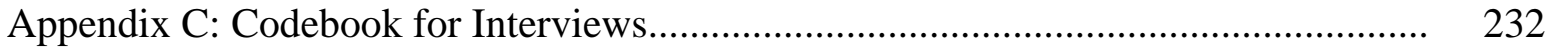

Appendix D: Informed Consent Agreements...................................................... 254

Appendix E: Demographics Questionnaire................................... 264

Appendix F: Debriefing Forms.................................................................. 266

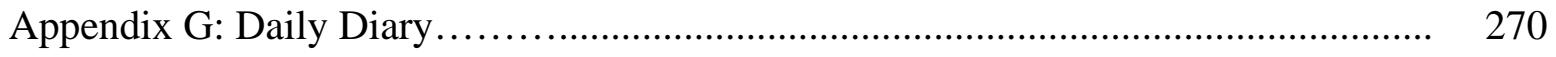




\section{General Introduction}

Generalized anxiety disorder (GAD) is characterized by excessive and uncontrollable worry, as well as several physical manifestations of anxiety, such as muscle tension, difficulty sleeping, and irritability (American Psychiatric Association [APA], 2013). The lifetime prevalence of GAD has been estimated to be 2.6\% in Canada (Statistics Canada, 2013) and 5.7\% in the United States (Kessler et al., 2005), making it one of the most common psychological disorders. GAD is associated with reduced work productivity and unemployment (Hunt, Issakidis, \& Andrews, 2002; Romera et al., 2011) and increased risk of physical illnesses, such as irritable bowel syndrome, pain disorders, and cardiac problems (Beesdo et al., 2009; Gros, Antony, McCabe, \& Swinson, 2009; Martens et al., 2010). GAD is also related to reduced social functioning (Henning, Turk, Mennin, Fresco, \& Heimberg, 2007), and diminished overall quality of life (Beard, Weisberg, \& Keller, 2010; Olatunji, Cisler, \& Tolin, 2007). Moreover, pathological worry is linked to substantial healthcare costs (Kujanpää, Ylisaukko-oja, Jokelainen, Linna, \& Timonen, 2014; Shirneshan et al., 2013); thus GAD produces considerable strain at the societal level.

GAD is a chronic disorder that does not typically remit unless treated (Bruce et al., 2005; Yonkers, Dyck, Warshaw, \& Keller, 2000). Although disorder-specific treatments for GAD lead to fairly high remission rates (typically 60\% to 70\%; Bolognesi, Baldwin, \& Ruini, 2014; Covin, Ouimet, Seeds, \& Dozois, 2008; Cuijpers et al., 2014; Hanrahan, Field, Jones, \& Davey, 2013), these rates still lag behind the remission rates that are achieved with evidence-based treatments for other anxiety disorders (see Stewart \& Chambless, 2009; Waters \& Craske, 2005 for reviews). In sum, there is substantial individual and societal burden associated with GAD, and a sizable proportion of individuals do not remit with current first-line treatments. Therefore, it is 
important that research efforts seek to expand the current theoretical and practical understanding of GAD, so that it might be more effectively assessed and treated.

Conceptualizing GAD has posed a significant challenge to researchers and clinicians alike, since the concerns of individuals with GAD are diffuse (i.e., worry about issues ranging from minor matters to death) and tend to shift over time (Dugas, Freeston, et al., 1998; Roemer, Molina, \& Borkovec, 1997). Thus, there is not a clearly discernible situation that people with GAD are afraid of that could lend itself to easily building a theory around. In the 1990s, a group of researchers at Laval University proposed that intolerance of uncertainty (IU) may be a common thread that underlies the array of diverse concerns described by people with GAD (Dugas, Gagnon, Ladouceur, \& Freeston, 1998; Freeston, Rhéaume, Letart, Dugas, \& Ladouceur, 1994; Ladouceur, et al., 1995). IU was first broadly defined as a tendency to have more negative cognitive, emotional and behavioural responses to uncertainty in everyday life situations (Freeston et al., 1994). The notion that IU may underpin pathological worry was based on clinical observations that difficulty accepting uncertainty was related to worrying about many different things (Freeston et al., 1994). The idea also stemmed from research demonstrating that people with high levels of worry interpreted ambiguous situations as threatening (Butler \& Mathews, 1987), and showed slower decision making in scenarios with unclear answers (Metzger, Miller, Cohen, Sofka, \& Borkovec, 1990; Tallis, Eysenck, \& Mathews, 1991). Of note, intolerance of uncertainty is thought to refer to a negative response to "not knowing" in the future, whereas intolerance of ambiguity is described as an aversion to "not knowing" in the here-and-now (see Rosen, Ivanova, \& Knäuper, 2014). Intolerance of ambiguity has been discussed and measured primarily in the nonclinical literature (Budner, 1962; Furnham \& Ribchester, 1995), whereas IU has been studied in the clinical psychology literature. IU is 
thought to be more specifically relevant to people with GAD than is intolerance of ambiguity (Freeston et al., 1994), as IU pertains to not knowing in the future, and the concerns of people with GAD are future-oriented (Borkovec, Robinson, Pruzinsky, \& DuPree, 1983; Sibrava \& Borkovec, 2006).

In 1998, Dugas, Gagnon, Ladouceur, and Freeston proposed the IU Model of GAD, and it remains one of the prominent theories of GAD (Behar, DiMarco, Hekler, Mohlman, \& Staples, 2009). The IU theory of GAD specifies that IU is the main cognitive process that underlies and maintains pathological worry directly and indirectly (Dugas et al. 1998; Dugas \& Robichaud, 2007). Directly, higher IU is theorized to lead to excessive worry because being intolerant of uncertainty may make even remote chances that negative events could occur difficult to accept; therefore, individuals higher in IU will be vulnerable to worry about any possibility that things could go wrong. Higher IU is also conceptualized to indirectly contribute to worry by fostering negative beliefs about problems and problem solving, positive beliefs about the usefulness of worry, and avoidance of anxiety-provoking mental images (Dugas et al., 1998).

A considerable body of research supports Dugas and colleagues’ (1998) IU Model of GAD. Scores on measures of IU, positive beliefs about worry, negative problem orientation, and cognitive avoidance are correlated with measures of GAD symptoms, and differentiate people with GAD from nonclinical controls (e.g., Dugas et al., 1998; Dugas et al., 2007; Freeston et al., 1994). In line with the theorized primary role of IU relative to other cognitive factors in Dugas and colleagues' theory, IU is more strongly related to GAD severity than other model components (Dugas et al., 1998); however, the same has since been found of negative problem orientation (Dugas et al., 2007). 
In addition, IU is more strongly related to worry than are other anxiety-related cognitive processes, such as perfectionism and need for control (Buhr \& Dugas, 2006). Research has also shown that people higher in IU interpret ambiguous situations as more worrisome than do individuals lower in IU (Koerner \& Dugas, 2008). Moreover, when IU is experimentally manipulated, worry levels change in the corresponding direction (Ladouceur, Gosselin, \& Dugas, 2000; Rosen \& Knäuper, 2009). Further, a GAD-specific cognitive behavioural treatment (CBT) that focuses primarily on addressing IU, from here on, CBT-IU (Dugas \& Robichaud, 2007; Robichaud, 2013) produces large, significant improvements in worry and other GAD symptoms (Dugas et al., 2003, 2010; Ladouceur et al., 2000). Lastly, over the course of CBT-IU, positive changes in IU come before decreases in worry (Dugas \& Ladouceur, 2000). In sum, the evidence to date suggests that IU is a key construct underlying excessive worry and that the IU Model of GAD (1998) is a helpful way to understand this disorder.

It is important to note that, although initially there was some evidence that IU was higher in people with GAD than in those with other anxiety disorders (e.g., Ladouceur et al., 1999), several studies have now demonstrated that IU may be high across the anxiety disorders (e.g., Boswell, Thompson-Hollands, Farchione, \& Barlow, 2013; Carleton et al., 2012; Holaway, Heimberg, \& Coles, 2006). In fact, recent research has shown that IU is elevated in a range of emotional problems, including depression (Gentes \& Ruscio, 2011; McEvoy \& Mahoney, 2012; Yook, Kim, Suh, \& Lee, 2010), eating disorders (Frank et al., 2012; Sternheim, Startup, \& Schmidt, 2011), and psychosis (White \& Gumley, 2010). Indeed, recent reviews and metaanalyses have recognized IU as a potential transdiagnostic cognitive process (e.g., Einstein, 2014; Gentes \& Ruscio, 2011). Supporting this, during transdiagnostic treatment of anxiety and depression, improvements in IU correspond with reductions in anxiety and depressive symptoms 
(Boswell et al., 2013), suggesting a link between IU and treatment outcomes across different disorders. IU has proved to be an important construct, not only in GAD, but in various psychological difficulties. The present dissertation focuses on IU in GAD as IU appears to be a central maintenance factor of pathological worry, the cardinal symptom of GAD (see Koerner \& Dugas, 2008), and a treatment that specifically targets IU (CBT-IU) significantly improves GAD symptoms with large effects (e.g., Dugas et al., 2003, 2010; van der Heiden, Muris, \& van der Molen, 2012).

To date, IU (as defined by Dugas and colleagues) has been assessed in the GAD literature primarily using a self-report measure called the Intolerance of Uncertainty Scale (IUS; French version: Freeston et al., 1994; English translation: Buhr \& Dugas, 2002). The items of the IUS were designed to assess the emotional and behavioural consequences of being uncertain, how being uncertain reflects on a person's character, expectations that the future be predictable and frustration when it is not, attempts to control the future, and "all-or-nothing” responses in uncertain situations (Freeston et al., 1994). The results of a large factor analysis $(N=2451)$ by Sexton and Dugas (2009) show that the IUS comprises two factors: Factor 1 reflects the belief that "uncertainty has negative behavioural and self-referent implications," and Factor 2 reflects the belief that "uncertainty is unfair and spoils everything." Examples of items that make up Factor 1 include: "When it’s time to act, uncertainty paralyses me," "Being uncertain means that I am not first rate," and "Uncertainty keeps me from living a full life.” Examples of items that make up Factor 2 include: “It’s unfair not having any guarantees in life,” “I can’t stand being taken by surprise," and "Unforeseen events upset me greatly.” The IUS has been shown to have excellent psychometric properties (Freeston et al., 1994). It also distinguishes likely GAD from non-GAD (Freeston et al., 1994), and detects changes in IU with treatment (Dugas et al., 2010). 
Overall, the IUS might be considered the backbone of research to date on IU in GAD, leading to studies that support theoretical conceptualizations of GAD and to an efficacious treatment for GAD that targets IU (Dugas \& Robichaud, 2007). Indeed, since the IUS has been used consistently to measure IU within the context of GAD, the construct of IU might be said to be defined by the IUS (Birrell, Meares, Wilkinson, \& Freeston, 2011).

Given the important role of the IUS in delineating the IU construct, it is important to critically examine what might be missing in how research has considered IU to date in order to advance the current understanding of GAD. One potential area for growth stems from the fact that the IUS was developed by clinician-researchers, based on knowledge from clinical work with individuals with GAD. Supporting this, the IUS has been described by Freeston, one of the main researchers involved in its development, as "largely atheoretical and derived from clinical opinion” (M. Freeston, personal communication, September 21, 2007, as cited in Birrell et al., 2011). Given this, gaining a more nuanced understanding of the IU construct from new perspectives, such as the viewpoint of individuals with GAD, is an important future research direction.

Moreover, it is crucial to compare the experience of being uncertain in people with GAD to people without a psychological disorder because of research suggesting that all individuals show some level of uncertainty aversion (Epstein, 1999; Halevy, 2007), and the fact that it is not yet clear what aspects of attitudes toward uncertainty are "normal" (versus "abnormal”) in people with GAD. A more thorough understanding of typical reactions to uncertainty is important in understanding the degree to which IU is pathological, and what might be expected of people with GAD following psychological treatment that aims to improve IU. 
Furthering knowledge about IU might also be achieved by using research methods other than closed-ended questionnaires, such as the IUS. Understanding more about what it is like to be uncertain by asking open-ended questions of people with GAD has the potential to advance what is known about IU, and to provide insights that could lead to improvements in the way IU is targeted in the treatment of GAD. Another way in which the treatment of IU might be advanced is by testing the degree to which specific strategies that are taught to people with GAD as part of CBT-IU lead to improvements in GAD symptoms and IU. It is important to test whether specific treatment strategies are having their intended effects so that they can be refined if they are not producing benefit, and it can also clarify the mechanisms through which treatment techniques have their effects. Given that $30 \%$ to $40 \%$ of people with GAD still do not fully benefit from first-line psychological treatments (Hanrahan et al., 2013), it is imperative that research continue to develop theories that have shown promise in improving the understanding of GAD, and to test strategies that may help people overcome this debilitating disorder.

The present dissertation sought to provide a deeper understanding of the IU construct and tested a treatment technique theorized to improve IU and GAD symptoms called "uncertainty recognition and exposure" (uncertainty exposure). In order to glean detailed information about IU, an in-depth mixed methods study was conducted to compare the experience of being uncertain from the perspectives of people with GAD and those of people without a psychological disorder (i.e., non-psychiatric control participants). This investigation was divided into two sets of research questions - one on attitudes toward uncertainty (Study 1a) and the other on behaviours in response to uncertainty (Study 1b). Study 1a examined novel questions about how individuals think about uncertainty and what uncertainty means to people, as well as attitudes toward different "types" of uncertainty, including how people think about uncertainty that can 
result in one of several positive outcomes. Study 1a also explored the viewpoints of people with GAD and non-psychiatric control participants on clinical observations that have been put forth in the GAD literature. Study 1b investigated unanswered questions about behaviours that people may employ in response to being uncertain, including examining what those behaviours are, their frequency, potential functions, discontinuation factors, and whether such behaviours are problematic.

Study 2 of the current dissertation used a quasi-experimental design to explore the effect of a single session of a treatment strategy known as uncertainty exposure on GAD symptoms and IU-related cognitions. This study also explored the importance of practicing uncertainty exposure outside of a lab or treatment context by investigating the association between homework compliance and improvement in GAD outcomes. Study 2 provides information about whether changing a person's behavioural responses to uncertainty (e.g., reassurance-seeking, excessive checking, avoiding uncertain situations) leads to changes in GAD symptoms and IUrelated cognitions, and therefore tests the proposed role of IU in maintaining GAD. Study 2 also offers information on the degree to which uncertainty exposure improves IU-related cognitions and GAD symptoms, informing the clinical utility of this treatment technique. Overall, the studies presented herein provide data that inform theoretical conceptualizations of the role of IU in GAD and the clinical application of this model. 


\section{Study 1a: Exploring Attitudes toward Uncertainty in People with GAD and Non- Psychiatric Control Participants}

\section{Introduction}

Intolerance of uncertainty (IU) is a central construct theorized to be involved in the development and maintenance of pathological worry - the main feature of generalized anxiety disorder (GAD; APA, 2013). The definition of IU has evolved over the past 2 decades (see Birrell et al., 2011), and has most recently been defined as "a set of negative beliefs about uncertainty and its implications” (Dugas et al., 2010). In research on GAD, IU has been assessed primarily by the self-report Intolerance of Uncertainty Scale (IUS; Buhr \& Dugas, 2002;

Freeston et al., 1994). Recently, an abbreviated version of the IUS, the IUS-Short Form (IU-12; Carleton, Norton \& Asmundson, 2007), was developed to study IU across psychopathologies (see Carleton et al., 2012 for more discussion). The psychometric properties of the IUS are excellent, and the IUS has yielded abundant data supporting the fundamental role of IU in GAD (see Carleton, 2012 for a review; Dugas et al., 2010). The IUS has also demonstrated strong clinical utility; it distinguishes likely GAD from non-GAD (Freeston et al., 1994), and detects changes in IU with treatment (Dugas et al., 2010).

The merits of the IUS notwithstanding, its items were developed by clinician-researchers, based on their experiences working with people with GAD (Freeston et al., 1994). One of the main developers of the IUS, Freeston, described the development of the IUS items as “atheoretical” and based on “clinical intuition” (M. Freeston, personal communication, September 21, 2007, as cited in Birrell et al., 2011). Furthermore, the IUS currently measures two behavioural responses to uncertainty (avoiding uncertainty and becoming paralyzed when uncertain), however, there are a wide range of other potential behaviours that individuals may 
engage in when they are uncertain (e.g., excessive information gathering when uncertain, reassurance seeking when uncertain).

A new measure of IU that was also developed by clinician-researchers called the Intolerance of Uncertainty Inventory (IUI; Gosselin et al., 2008; replication in an English speaking sample: Carleton, Gosselin, \& Asmundson, 2010) builds on the IUS by assessing a greater range of cognitive and behavioural manifestations of IU. However, this measure still addresses only three cognitive and three behavioural manifestations of IU. ${ }^{1}$ Given that the items of the current measures of IU were not generated by individuals with GAD directly (as in the mixed method approaches to scale construction) and may not encompass the full range of responses to uncertainty, it is unclear whether the IUS and IUI truly capture the experience of being uncertain from the perspective of people with GAD. As such, asking people with GAD about their experience of uncertainty directly has the potential to unearth new information about IU, and contribute to current IU theories and assessment.

Further, although current measures of IU such as the IUS give an indication of the degree to which various responses to uncertainty (e.g., feeling paralyzed by uncertainty, feeling that uncertainty keeps one from living a full life) are typical of a respondent; the current self-report measures of IU do not provide insight about why respondents endorse items. That is, an individual may strongly endorse the item “uncertainty makes life intolerable” on the IUS, however, it remains unknown what makes uncertainty so intolerable to that person. Thus, although studies using self-report measures of IU have made clear that individuals with GAD are bothered by uncertainty (Carleton et al., 2012; Dugas et al., 1998, 2007; Starcevic \& Berle,

\footnotetext{
${ }^{1}$ The manifestations of uncertainty assessed in the Intolerance of Uncertainty Inventory are: avoidance of uncertainty, reassurance seeking, attempts to control uncertainty, overestimation of the probability of negative events, doubt in uncertain situations, and worry in uncertain situations (Gosselin et al., 2008).
} 
2006), it remains unknown precisely why this is the case. Knowing more about beliefs underlying IU may contribute to a deeper understanding of people with GAD, and has the potential to enhance current CBT treatments that target IU by highlighting beliefs that may be particularly important to challenge.

Moreover, it appears that in GAD research it has been assumed that uncertainty is a unitary construct, and individuals respond the same to uncertainty regardless of contextual factors that surround it. For instance, the IUS does not assess attitudes toward uncertainty with a high versus low likelihood of resolving negatively. Relatedly, clinicians are not guided to ask people with GAD about the factors that make uncertainty more or less tolerable. As such, there is not yet an understanding of whether all types of uncertainty "feel the same" to individuals with GAD. This is interesting, because in the assessment and treatment of other anxiety disorders, contextual factors are explicitly considered (see Abramowitz, Deacon, \& Whiteside, 2011 for a review). For example, a person with social anxiety disorder might endorse a general fear of first time meeting situations, but further assessment would occur to determine whether the fear is worse when the person is being introduced to one new individual, versus a group of individuals. Within the context of GAD, I propose that it is similarly important to understand whether there are factors that make the experience of being uncertain more or less anxiety-provoking, as this may inform treatments that involve approaching uncertainty for the purpose of improving IU.

Another important way to extend the current knowledge of IU is to more fully understand the ways in which the experience of uncertainty in people with GAD is the same as and different from the experience of non-psychiatric control participants. Findings from the nonclinical literature have shown that although there are vast individual differences in preferences for certainty (Etner, Jeleva, \& Tallon, 2010), on the whole, most people prefer certainty to 
uncertainty (Epstein, 1999; Halevy, 2007). Indeed, strategic decision-making theorists have suggested that greater certainty allows for more informed planning, decision making, and actions (Corbin, 1980; Orasanu \& Connolly, 1993; Thompson, 2003). These findings suggest that IU may not be an entirely pathological cognitive process, and that the attitudes of people with and without GAD may be similar in some respects. Although research suggests that IU is elevated in individuals with GAD compared to individuals without a psychological disorder (non-psychiatric control participants [NPCs]; Carleton et al., 2012; Dugas, et al., 1998), how individuals with GAD differ from NPCs on IU has not yet been examined. In other words, it is unclear whether the difference between people with and without GAD is merely a matter of degree, or if there are also qualitative differences in the way that individuals with and without GAD experience uncertainty. Knowing more about how the experience of being uncertain differs in individuals with GAD compared to non-psychiatric control participants would point to what aspects of the uncertainty response is "normal" in people with GAD. This information could guide clinicians by highlighting which aspects of IU to focus on in GAD treatment, and would also provide a sense of what to expect in terms of attitudes toward uncertainty following treatment. For example, people with GAD may continue to have negative beliefs about some forms of uncertainty by the end of treatment, if even non-psychiatric control participants are somewhat intolerant of uncertainty. Relatedly, knowing how non-psychiatric control participants respond to uncertainty may help clinicians normalize attitudes toward uncertainty in people with GAD. Understanding what constitutes a healthy attitude toward uncertainty may also provide ideas about adaptive ways to cope with uncertainty that could be taught to people with GAD. 


\section{Present Study}

The present study used a mixed methods approach that involved asking people with GAD and NPCs open-ended questions about uncertainty in an interview format. This method allowed for novel questions to be posed that have not yet been measured by instruments in the literature. Furthermore, the open-ended nature of questions allowed people with GAD and NPCs to provide any possible response; allowing for questions about uncertainty to be more fully understood from the perspective of those who experience high and low levels of IU. Mixed qualitative and quantitative approaches such as the current method are becoming increasingly popular because they reduce researcher inference and promote discovery (see Teddlie \& Tashakkori, 2009). Indeed, several studies in the field of anxiety disorders have recently employed mixed methods to gain original insights about phenomena of interest, such as therapy experiences of people with GAD (Marcus, Westra, Angus, \& Kertes, 2011), the function of reassurance-seeking in OCD (Parrish \& Radomsky, 2010), cessation factors of panic attacks (Radomsky, Rachman, Teachman, \& Freeman, 1998), feared consequences of panic attacks (Raffa, White, \& Barlow, 2004), and the experience of pain in people with health anxiety (Tang et al., 2009).

The present study focused on understanding attitudes toward uncertainty in people with GAD compared to NPCs. There are many definitions of attitudes (see Kraus, 1995), and it has been suggested that attitudes may have cognitive, emotional, and behavioural components (Breckler, 1984; LaPiere, 1934). In the present study, the use of the term “attitude” refers primarily to the cognitive aspect of this construct, which encompasses: "beliefs, knowledge structures, perceptual responses, and thoughts” (Breckler, 1984, p. 1191). Questions about attitudes toward uncertainty that have not been addressed in research to date include: 1) what thoughts people with GAD spontaneously report when uncertain, and reasons why people with GAD endorse negative beliefs about uncertainty (e.g., reasons why uncertainty is intolerable); 2) 
whether specific factors (e.g., the probability or cost associated with potential negative outcomes) change how people with GAD experience uncertainty; 3) the attitudes of people with GAD toward uncertain situations in which outcomes are most likely positive or neutral; and 4) the degree to which people with GAD relate to clinical observations that researchers have made about IU and worry. In order to understand the attitudes of individuals with GAD in the context of what is normative, questions about these four topics were investigated in people with GAD and non-psychiatric control participants. Reasons why these particular objectives were selected for study, along with hypotheses that could be made based on the extant literature are described below. However, it is of note that the current study was intended to be primarily hypothesis generating, as opposed to hypothesis testing, in the spirit of uncovering novel insights about IU in GAD and ideas for future research. Thus, original themes in response to questions that were not anticipated a priori were expected to emerge.

\section{Objective 1. Thoughts and Underlying Beliefs Related To Being Uncertain}

Thoughts when uncertain. The IUS has been the primary instrument through which cognitions related to uncertainty have been studied in people with GAD. The information gathered using the IUS has been fundamental in arriving at the current theoretical understanding of the association of IU to GAD, and in developing a corresponding treatment for GAD that targets IU (see Birrell et al., 2011; Gentes \& Ruscio, 2011). Despite the useful information that the IUS has provided, the items of the IUS were generated by researchers based on their clinical experience with people with GAD (Freeston et al., 1994); thus, it is unclear whether the IUS fully and accurately captures what it is like to be intolerant of uncertainty from the perspective of people with GAD. Thus, it was of interest to directly ask individuals with GAD and NPCs about thoughts that they have when they are uncertain. This approach has the potential to provide new 
information about the thought patterns of people with GAD that have not yet been identified by existing methods, such as self-report questionnaires, potentially highlighting ways of thinking that link IU and worry that have not yet been considered. Furthermore, learning how NPCs think when they are uncertain may highlight healthy ways of considering uncertainty that could potentially be taught to people with GAD in treatment. Finally, learning more about how people with GAD and NPCs think when uncertain can inform future research seeking to refine and expand on current measures of IU.

Given that people with GAD hold beliefs about uncertainty that are more negative than do individuals low in worry and anxiety (e.g., Carleton et al., 2012; Dugas et al., 1998, 2007; Lee, Orsillo, Roemer, \& Allen, 2010; van der Heiden et al., 2012), it was expected that more people with GAD would report negative thoughts about uncertainty compared to NPCs. As no studies have yet asked participants with GAD or NPCs to describe their thoughts in an uncertain situation in an open-ended manner, no other hypotheses were proposed.

The belief that uncertainty is negative. People with GAD endorse items on the IUS that reflect the notion that uncertainty is negative (e.g., "uncertainty makes life intolerable”; "uncertainty keeps me from living a full life”; "I must get away from all uncertain situations”; Dugas et al., 2007; Freeston et al., 1994; Lee et al., 2010; van der Heiden et al., 2012). However, it is currently not well understood why people with GAD find uncertainty so unacceptable. Obtaining a deeper understanding of why uncertainty is intolerable to people with GAD could provide a better understanding of how to help people improve IU in GAD treatment. Moreover, knowing why uncertainty is negative to individuals with GAD may lead to a more thorough theoretical understanding of how IU maintains worry, and may point to ways in which the IU model could be linked to other theories of GAD. 
There were a few predictions made about why people with GAD find uncertainty negative. Research has suggested that uncertainty can interfere with planning and decision making (Corbin, 1980; Orasanu \& Connolly, 1993; Thompson, 2003). Also, studies have shown that compared to nonanxious individuals, people with GAD overprepare when uncertain (BeesdoBaum et al., 2012) and are slower to make decisions when uncertain (Ladouceur, Talbot, \& Dugas, 1997). As such, it was hypothesized that more people with GAD than NPCs would describe being unable to plan or prepare as a reason why uncertainty is negative.

Relatedly, previous studies have shown that anxious individuals display a more exaggerated emotional and cortical response to uncertainty than do nonanxious individuals (see Simmons et al., 2013; Simmons, Strigo, Matthews, Paulus, \& Stein, 2006). Indeed, Grupe and Nitschke (2013) concluded that "negative anticipatory emotions make uncertainty particularly intolerable for anxious people” (p. 496). Therefore, it was hypothesized that compared to NPCs, a greater proportion of people with GAD would report their own strong responses to uncertainty as a reason for viewing uncertainty negatively.

Personal meanings of uncertainty. The IUS includes several items that reflect negative personal meanings of uncertainty such as "being uncertain means that I lack confidence," "being uncertain means that I am not first rate," and "being uncertain means that a person is disorganized" (Buhr \& Dugas, 2002). However, the IU theory of GAD is silent on these personal meanings, which is interesting given that self-meanings are considered important in cognitive theory, as they are thought to bias information processing and sustain negative emotion (e.g., Beck, Emery, \& Greenberg, 1985; Clark \& Beck, 1989, 2010). Further knowledge of the personal meanings of uncertainty may help clinicians to better understand and challenge 
unhelpful beliefs about uncertainty in GAD clients, and could provide a deeper theoretical understanding of how beliefs related to uncertainty maintain excessive worry.

Given that individuals with GAD may have negative beliefs about themselves (HazlettStevens, 2008; Leahy, 2006), and are generally more bothered by uncertainty than are healthy individuals (e.g., Carleton et al., 2012; Dugas et al., 2007), it was hypothesized that more people with GAD than NPCs would report that uncertainty has negative personal meanings. In addition, since people with GAD score higher on items of the IUS assessing the belief that being uncertain means that one is unconfident, inadequate (i.e., not "first rate"), and disorganized, it was hypothesized that these themes may arise as personal meanings of being uncertain. It was also suspected that self-beliefs related to the theme of being incompetent (“I’m inept”; “I can’t cope”), which have been identified in clinical writings on GAD (Hazelett-Stevens, 2008; Leahy, 2006), may emerge as personal meanings of uncertainty.

The belief that uncertainty is unfair. The IUS contains a number of items reflecting the notion that uncertainty is unfair (see Buhr \& Dugas, 2002). However, the reasons for viewing uncertainty as unfair have not yet been studied in people with GAD. Knowing more about why uncertainty is perceived as unfair to people with GAD and if this differs from the views of nonpsychiatric control participants would be useful in clarifying ways of thinking that may underpin pathological worry. Furthermore, anger has been found to be elevated in people with GAD relative to individuals low in worry (Erdem, Çelik, Yetkin, \& Özgen, 2008; Deschênes, Dugas, Fracalanza, \& Koerner, 2012), and attributions of unfairness can elicit anger (Batson et al., 2007; Horan, Chory, \& Goodboy, 2010; Smith \& Ellsworth, 1985). Thus, a more thorough understanding of why uncertainty is unfair to people with GAD may assist with understanding anger in those with higher worry. 
Findings from the business and social psychology literatures have investigated the association between uncertainty and unfairness. One highly replicated finding is that under conditions of uncertainty, fairness becomes more important to people, presumably because fairness helps people to manage uncertainty by reducing confusion about what to expect (see Van den Bos \& Lind, 2013 for a review). Along these lines, "self-uncertain individuals," defined as people who are bothered by "a subjective sense of doubt or instability in self-views and world-views” (Van den Bos \& Lind, 2013, p. 124), tend to have larger affective, cognitive, and behavioural responses to unfair procedural information than do people who are more certain of themselves (Van den Bos \& Lind, 2002, 2013). Research has also shown that unexpected negative information, such as an employer not following through on a contract, or a sudden price increase, may be perceived as more unfair than expected negative information (e.g., Brockner, Typler, \& Cooper-Schneider, 1992; Morrison \& Robinson, 1997; Robinson \& Rousseau, 1994; Xia, Kukar-Kinney, \& Monroe, 2010). Thus, unfairness may be more salient to people when they are uncertain, particularly when people are not expecting to be uncertain.

Since people with GAD go to great lengths to ensure certainty, frequently reducing uncertainty through actions such as excessive information gathering or overpreparing (BeesdoBaum et al., 2012), people with GAD may have higher expectations of certainty than others (Fracalanza, Koerner, Deschênes, \& Dugas, 2014). The excessive efforts that people with GAD put forth to prevent uncertainty may make unexpected events that occur despite the best of planning feel particularly undeserved (Fracalanza et al., 2014). Thus, it was predicted that more people with GAD than NPCs would describe uncertainty as unfair because they don't deserve to be uncertain. 
Beliefs about coping abilities and outcomes when uncertain. The present study sought to explore if people with GAD, when they are uncertain, assume that they will not be able to cope (Borkovec, 1994; Davey \& Levy, 1998; Hazlett-Stevens \& Craske, 2003; Koerner \& Dugas, 2008; Robichaud \& Dugas, 2005) and that situations will inevitably turn out poorly (MacLeod, Williams, \& Bekerian, 1991; Miranda \& Mennin, 2007; Provencher, Freeston, Dugas, \& Ladouceur, 2000). Although these assumptions have already been observed in people with GAD, it is not known whether these assumptions are activated within the context of uncertainty, specifically. It was hypothesized that compared to NPCs, a greater proportion of individuals with GAD would describe a tendency to believe they are unable to cope when uncertain, and a tendency to be pessimistic about outcomes when uncertain.

\section{Objective 2. Factors That May Moderate Attitudes toward Possible Negative Outcomes}

The present study examined factors that may change the way that people with GAD and NPCs respond to potential negative outcomes. To date, there has been an implicit assumption that individuals with GAD find any form of uncertainty unacceptable (see Carleton, 2012;

Ladouceur, et al., 2000). However, research has not yet systematically investigated whether this is the case. Related to this, research in GAD has treated uncertainty as a unitary construct, when there is ample evidence in nonclinical disciplines that uncertainty is a multidimensional construct (i.e., there are different kinds of uncertainty; Chow \& Sarin, 2002; Kahneman \& Tversky, 1982; Knobloch \& Solomon, 1999; Miller, 2012). Knowing more about whether all types of uncertainty are equally intolerable to people with GAD would help provide a more precise definition of what it means to be intolerant of uncertainty, and would also point to just how “intolerant” of uncertainty people with GAD are by offering initial information on whether people with GAD are intolerant of uncertainty regardless of its context. This information may be 
important in distinguishing "normal” IU from more pathological IU, in the sense that intolerance of uncertainty regardless of contextual factors surrounding it may be more unhelpful to a person than aversion to specific forms of uncertainty. Several potential moderators of attitudes toward uncertainty were examined in this study, including probability of a potential negative outcome, cost of a potential negative outcome, whether others are also uncertain versus being the only one uncertain, and controllability over a potential negative outcome. A description of each of these possible moderators of attitudes toward uncertainty and any hypotheses that could be made based on the extant literature are described below.

Probability and cost. Cognitive theories suggest that worry and anxiety are triggered by perceptions of threat, which comprise estimations of how likely and severe possible negative outcomes are (Beck et al., 1985; Borkovec, Alcaine, \& Behar, 2004; Mathews, 1990). Individuals with GAD overestimate the probability of feared outcomes occurring, and how bad these outcomes would be if they occurred (Borkovec, Hazlett-Stevens, \& Diaz, 1999; Butlers \& Mathews, 1983, 1987; Mitte, 2007). Moreover, clinical observation suggests that even when the probability or cost of potential negative events are low, people with GAD say that they would still worry (Dugas, Buhr, \& Ladouceur, 2004; Dugas, Freeston, \& Ladouceur, 1997; Dugas, Gosselin, \& Ladouceur, 2001; Dugas \& Koerner, 2005; Dugas \& Robichaud, 2007). In other words, people with GAD appear to have difficulty tolerating even miniscule levels of potential future threat.

Contrary to this, however, current cognitive restructuring strategies that are used in CBT for GAD challenge how likely and severe potential negative outcomes are (e.g., Craske \& Barlow, 2006). The rationale for challenging perceptions of likelihood and cost is that lower probability and cost estimates of negative events will reduce worry (e.g., Craske \& Barlow, 
2006). In sum, even though theoretically individuals with GAD worry regardless of the probability and cost of a potential negative outcome, in practice, individuals with GAD are being asked to challenge and reappraise the probability and cost of possible unfavourable events. Thus, it is important to begin to explore whether lower perceptions of the probability and cost of uncertain events actually leads individuals with GAD to worry less about hypothetical outcomes.

Based on theory and clinical observations (e.g., Dugas et al., 1997, 2004; Dugas \& Robichaud, 2007), it was expected that individuals with GAD would describe negative attitudes toward uncertainty regardless of the probability or cost of the uncertain event, whereas nonpsychiatric control participants were expected to describe less negative attitudes toward uncertainty when the probability or cost of the uncertain event was lower.

Uncertainty of others. Research in the nonclinical literature on uncertainty has suggested that individuals' attitudes differ depending on the parameters of the potential negative event in question. For example, Chow and Sarin (2002) found that being uncertain when all other individuals are also in the dark (unknowable uncertainty) is more tolerable to nonclinical individuals than is being the only one uncertain when others have information that would allow for certainty (knowable uncertainty). Given that there are no available data on how individuals with GAD respond to unknowable versus knowable uncertainty, hypotheses were left exploratory.

Controllability. Research has suggested that in healthy and anxious populations alike, situations that are perceived as more controllable are experienced as less stressful (Barlow, 2002; Başoğlu \& Mineka, 1992; Foa, Zinbarg, \& Rothbaum, 1992; Kemeny, 2003; Miceli \& Castelfranchi, 2005; Rapee, Craske, Brown \& Barlow, 1996). Zvolensky, Heffner, Eifert, Spira, and Fledner (2001) have extended this idea to ambiguous situations, showing that in a 
nonclinical sample, higher scores on a measure assessing perceived lack of control over anxietyrelated events (e.g., believing one cannot escape from a frightening situation) was associated with more threatening interpretations of ambiguous situations. It is of note that Zvolensky et al. studied ambiguous situations, which are events happening in the here-and-now that can be interpreted in numerous ways, whereas the current study focused on understanding uncertain situations, which are scenarios with numerous possible future outcomes (Rosen et al., 2014). Nevertheless, Zvolensky's findings in a nonclinical sample point to the possibility that not knowing (whether in the moment or in the future) may be less threatening when one perceives a higher degree of control. However, the degree to which control over an uncertain situation impacts how being uncertain is experienced by people with GAD has not yet been examined; thus hypotheses about this question were left open.

\section{Objective 3. Exploring Uncertainty as Positive or Neutral}

When is uncertainty positive? To date, no studies have examined the circumstances, if any, under which not knowing what the future has in store can be positive in the eyes of people with GAD. Knowing more about conditions under which uncertainty is positive to individuals with GAD would add to the understanding of whether people with GAD respond differently to uncertainty depending on the "type" of uncertainty. This may also inform the IU theory of GAD by testing whether there are exceptions to the rule that uncertainty is intolerable to people with GAD. These data also have the potential to generate ideas about forms of uncertainty that practitioners may ask people with GAD about in order to facilitate more flexible thinking about uncertainty (i.e., the idea that not all uncertainties are equally "bad”).

Nonclinical research has described the benefits of uncertainty, including the notion that uncertainty is exciting, motivating, and promotes learning. A number of experiments in 
nonclinical samples have shown that uncertainty about positive outcomes produces positive feelings and arousal (i.e., excitement; Bar-Anan, Wilson, \& Gilbert, 2009; Lee \& Qiu 2009; Schultz, Dayan, \& Montague 1997). Further, in response to an open-ended question, nonclinical participants reported that guessing outcomes before they are known is exciting (e.g., guessing the sex of an unborn baby; Brun \& Teigen, 1990). Experiments in nonclinical samples have also demonstrated that when people are pursuing a reward, uncertainty about how big the reward will be (e.g., $50 \%$ chance of $\$ 1$ or $\$ 2$ ) is not only more exciting but is also more motivating than knowing exactly what the reward is (e.g., definitely \$1; Shen, Fishbach, \& Hsee, 2014). Further, because human beings are motivated to resolve feelings of uncertainty (e.g., Berger \& Calabrese, 1975; Inglis, 2000), which may be accomplished by thinking at length about the ways in which the situation could resolve, Tormala, Jia, and Norton (2012) theorized that people may think more deeply about uncertain information than they do about absolute facts, potentially facilitating greater processing and learning. Similar to this, people have been found to recall more information about incongruent than congruent messages (Maheswaran \& Chaiken, 1991), suggesting deeper processing of information that is unexpected. Thus, it was hypothesized that non-psychiatric control participants would describe themes that uncertainty could be exciting and motivating, and could lead to learning. However, because reasons for uncertainty being positive have not yet been investigated in people with GAD, predictions about the responses of people with GAD were left exploratory.

Attitudes toward uncertainty about positive or neutral events. There is currently a limited understanding of the attitudes of people with GAD toward uncertainty that likely will result in a favourable or neutral outcome. That is, it is not clear how people with GAD respond to not knowing how something will resolve when all potential options are relatively positive (or, 
not "bad") (e.g., uncertainty about receiving a gift). Along these lines, it is unknown whether people with GAD are intolerant only of uncertainty that has the potential to resolve negatively, or are also intolerant of uncertainty itself, irrespective of the outcome. Overall, a stronger empirical understanding of attitudes toward uncertainty about positive or neutral events in people with GAD would inform the extent to which this population is actually intolerant of uncertainty (i.e., intolerant of all forms of uncertainty), helping to clarify the IU construct. Furthermore, information on responses to uncertainty about positive or neutral events could inform GAD treatment in that this information could point to forms of uncertainty that individuals with GAD may benefit from systematically approaching in the course of CBT.

A few studies to date may inform predictions about how people with GAD react to uncertainty about positive events. Kupor, Tormala, and Norton (2014) conducted two experiments and found that on average, people in a nonclinical sample showed more interest in and a greater desire to try an activity that is likely to have a positive outcome when they read a review framed in uncertain terms (e.g., "it could become a top dining fixture in the area") versus certain terms (e.g., "it has become the top dining fixture in the area"). However, this effect was moderated by IU, in that only people with lower IU showed more interest in and a greater desire to try a restaurant or comedy club with an uncertain versus certain review, whereas people with higher IU showed the same level of desire to try a dining or comedy establishment regardless of how the event was framed, and showed lower interest in a dining or comedy establishment framed in uncertain versus absolute terms. These findings suggest that people lower in IU may perceive uncertainty that is likely to resolve favourably in a more positive way relative to people higher in IU. 
Relatedly, Byrne, Hunt, and Chang (2015) conducted a study wherein nonclinical participants completed the IUS and then rated their worry and perceptions of the probability and cost of a negative outcome in four types of situations: (1) ambiguous situations with the potential for negative outcomes (e.g., "My lecturer calls me to their office to discuss the quality of my work over the last few weeks.”), (2) unpredictable positive situations without the potential for negative outcomes (e.g. "My friend gives me a letter and tells me it contains the best news I am going to receive all year. I am sure it will be good news, but I cannot imagine what it is.”), (3) positive scenarios with definite outcomes, and (4) negative scenarios with definite outcomes. Byrne et al. used multilevel modeling to investigate whether people higher in IU showed more worry in scenarios with ambiguous or unpredictable outcomes versus certain outcomes. They found that there was a stronger relationship between IU and worry in ambiguous situations and unpredictable positive situations than there was in absolute positive or negative situations, with no differences in the strength of the association between IU and worry in ambiguous situations versus unpredictable positive situations. Using path analyses, Byrne et al. also tested whether perceptions of the probability and cost of a negative outcome (multiplied to make a single "threat perception” variable) explain the association between IU and worry in ambiguous or unpredictable positive scenarios. The authors found that threat perception explained a significant, moderately sized variance (61\%) in the relationship between level of IU and worry in ambiguous scenarios. On the other hand, threat perception explained a significant but small-sized variance (28\%) in the relationship between level of IU and worry in unpredictable positive scenarios. The authors interpreted these findings to suggest that threat perceptions explain some of the connection between IU and worry in situations with possible negative outcomes, and that IU is also associated with worry in situations with various positive outcomes, and most of this 
relationship is not accounted for by threat perceptions. Thus, people with GAD may worry in response to uncertain situations without the potential for negative outcomes.

Based on the limited data to date on how uncertainty about positive events is perceived by people with high IU, more people with GAD compared to NPCs were expected to report negative emotions in response to uncertainty about positive events (e.g., uncertainty about receiving a gift). In addition, more people with GAD compared to NPCs were expected to report being bothered by uncertainty itself, and not just uncertainty about possible negative outcomes.

\section{Objective 4. Systematically Investigating Clinical Observations}

One observation that Dugas et al. (2004) have documented is that some clients with GAD say that they would prefer to "just know," even if the outcome is negative, rather than remain uncertain. Demonstrating this, Dugas et al. included the following quote from a client in their book chapter: "I would prefer that my husband just leave me now than not know how things will work out.” Another observation in Dugas and Robichaud's (2007) CBT-IU manual is that people with GAD appear to be "allergic to uncertainty," reacting more intensely than others to even small amounts of uncertainty. Although these reflections are based on interactions with GAD clients, and are theoretically sound, the degree to which these descriptions resonate with individuals with GAD compared to non-psychiatric control participants has not yet been systematically investigated.

People higher in anxiety show a tendency to select smaller rewards that are more probable over bigger rewards that are less probable (e.g., Raghunathan \& Pham, 1999). Moreover, individuals higher in IU show a preference for smaller immediate rewards over having to wait for larger rewards (Luhmann, Ishida, \& Hajcak, 2011). Clinical observations similarly suggest that some individuals with GAD prefer negative information that is certain than waiting 
to hear an outcome (Dugas et al., 2004). Likewise, there is some evidence that under some circumstances, even individuals in nonclinical samples would prefer a certain negative outcome to not knowing (Lieberman, Cathro, Nicol, \& Watson, 1997; Loewenstein, Weber, Hsee, \& Welch, 2001).

Given that individuals with GAD are known to have more difficulty tolerating uncertainty than nonclinical controls (Carleton et al., 2012; Lee et al., 2010), it was expected that a higher proportion of people with GAD than NPCs would prefer a sure negative outcome to an uncertain one, although this theme was also expected to be described by some non-psychiatric control participants. In addition, given clinical evidence (Dugas \& Robichaud, 2007) and research on IU in GAD (e.g., Carleton et al., 2012), it was hypothesized that a greater proportion of people with GAD would report being "allergic" to uncertainty compared to non-psychiatric control participants. 


\section{Method}

An in-depth mixed methods study was conducted and was divided into two subinvestigations - one on attitudes toward uncertainty (the present study) and the other on behaviours in response to uncertainty (Study 1b of this dissertation).

\section{Participants}

Participants were recruited via flyer advertisements posted in the Toronto community and on online forums (e.g., Kijiji; see Appendix A for advertisements). All interested individuals were invited to complete a telephone screen to determine eligibility. The telephone screen comprised the Mini International Neuropsychiatric Interview (MINI) Screen, and corresponding modules of the MINI Interview when gateway questions from the MINI Screen were endorsed. Individuals were eligible for the present study if they were between the ages of 18 and 65 years and they: 1) reported symptoms that met the diagnostic criteria for DSM-IV-defined GAD on the MINI Interview, and did not report symptoms of another psychological disorder more severe than GAD on the MINI (GAD group); or 2) did not report symptoms that met the diagnostic criteria for any current psychological disorder on the MINI (NPC group). The present study began before the release of DSM-5 (APA, 2013); however, the core diagnostic criteria for GAD have not changed from DSM-IV to DSM-5 (Starcevic \& Portman, 2013). Therefore, although the GAD group was selected in the present study by assessing DSM-IV-defined GAD symptoms, the symptoms of GAD are the same in DSM-5, except for the hierarchical rules (removed in DSM-5) requiring that GAD not be diagnosed when the symptoms occur exclusively during the course of a mood disorder, psychotic disorder, pervasive developmental disorder, or posttraumatic stress disorder. No participants were excluded based on the hierarchical rules present in DSM-IV but not DSM-5. In total, 117 individuals were screened, and 40 met the inclusion criteria, including 20 per group. Individuals were excluded at the time of the phone 
screen for reporting elevated worry that did not meet the full diagnostic criteria for GAD (52\%), or symptoms that met the diagnostic criteria for a principal diagnosis other than GAD (47\%). One additional individual (1\%) was excluded after data collection, as information provided on questionnaires completed during the laboratory visit differed from information provided at the time of the telephone screen, and the participant's eligibility could not be determined.

Individuals with comorbid diagnoses were included in the present study to increase the external validity of results (Newman, Przeworski, Fisher, \& Borkovec, 2010). In the present sample, $65 \%$ of the GAD group had no comorbid disorder, $15 \%$ had one comorbid disorder, $15 \%$ had two comorbid disorders, and 5\% had three or more comorbid disorders. Additional diagnoses in the GAD group included social anxiety disorder (SAD; 50\%), major depressive disorder (MDD; 35\%), obsessive-compulsive disorder (OCD; 20\%), panic disorder (5\%), specific phobia (5\%), and bulimia nervosa (5\%). The GAD group included three individuals who were taking psychotropic medication, and four who were receiving psychotherapy (participants were not asked what type of psychotherapy they were receiving). Individuals receiving anxiety treatment were included in the present study as the goal was to understand the perspectives of a wide range of individuals with GAD (i.e., individuals with diverse characteristics), as per qualitative research guidelines (Flick, 2009).

The sample was $75 \%$ female and $25 \%$ male, between the ages of 19 to 64 years old ( $M=$ 30.70, $S D=12.92)$. The sample was ethnically diverse, with individuals self-identifying as: White (46\%), South Asian (23\%), East Asian (10\%), South East Asian (8\%), Arab/West Asian (5\%), Multiethnic (5\%), and Black (3\%). More information about demographics can be found in Table 1. 
Table 1

Demographic Characteristics by Group $(N=40)$

\begin{tabular}{|c|c|c|c|}
\hline Characteristic & $\begin{array}{c}\text { GAD } \\
(n=20)\end{array}$ & $\begin{array}{c}\text { NPC } \\
(n=20)\end{array}$ & $t$ or $\chi^{2}$ \\
\hline Age in years - M $(S D)^{a}$ & $25.78(9.80)$ & $33.60(15.75)$ & -1.81 \\
\hline Sex - Frequency (\%) & & & - \\
\hline Female & $15(75 \%)$ & $15(75 \%)$ & \\
\hline Male & $5(25 \%)$ & $5(25 \%)$ & \\
\hline Race/Ethnicity - Frequency (\%) & & & 5.42 \\
\hline White & $9(45 \%)$ & $9(47.4 \%)$ & \\
\hline South Asian & $5(25 \%)$ & $4(21.1 \%)$ & \\
\hline East Asian & $2(10 \%)$ & $2(10.5 \%)$ & \\
\hline South East Asian & $1(5 \%)$ & $2(10.5 \%)$ & \\
\hline West Asian/Arab & $2(10 \%)$ & $0(0 \%)$ & \\
\hline Black & $1(5 \%)$ & $0(0 \%)$ & \\
\hline Mixed Race & $0(0 \%)$ & $2(10.5 \%)$ & \\
\hline Marital Status - Frequency (\%) & & & 2.86 \\
\hline Single & $14(70 \%)$ & $13(65 \%)$ & \\
\hline Married/Common-law & $4(20 \%)$ & $7(35 \%)$ & \\
\hline Divorced/Widowed & $2(10 \%)$ & $0(0 \%)$ & \\
\hline Enrolled in Education Program - Frequency (\%) & & & 1.04 \\
\hline University & $11(55 \%)$ & $12(60 \%)$ & \\
\hline College & $1(5 \%)$ & $1(5 \%)$ & \\
\hline Adult Education & $1(5 \%)$ & $0(0 \%)$ & \\
\hline Not Enrolled in Education Program & $7(35 \%)$ & $7(35 \%)$ & \\
\hline Employment Status - Frequency (\%) & & & 0.48 \\
\hline Not Working (including student) & $8(40 \%)$ & $9(45 \%)$ & \\
\hline Working part-time & $7(35 \%)$ & $5(25 \%)$ & \\
\hline Working full-time & $5(25 \%)$ & $6(30 \%)$ & \\
\hline
\end{tabular}

Note. GAD = Generalized anxiety disorder; NPC = Non-psychiatric control (i.e., no psychological disorder). The data provided on age were not normally distributed (Shapiro-Wilk test $=0.83, p<.05$ ); therefore, a non-parametric test was also conducted that does not assume normality and results showed no significant difference between groups on age (Mann-Whitney U $=139.50, p=.24) .{ }^{\mathrm{a}}$ Two participants in the GAD group chose not to report their age; ${ }^{\mathrm{b}}$ One participant in the NPC group chose not to report their race/ethnicity. 
In terms of clinical characteristics, the GAD group scored significantly higher than the NPC group on the IUS and all symptoms assessed ( $p s<.01$; see Table 2 ), confirming expected differences between groups. Almost all individuals (90\%) in the GAD group scored above the original cut-score of 5.7 used to determine GAD on the Generalized Anxiety Disorder Questionnaire (GAD-Q-IV; Newman et al., 2002), and 80\% scored above a new, more stringent cut-score of 7.67 on the GAD-Q-IV (Moore, Anderson, Barnes, Haigh, \& Fresco, 2014).

In addition, $95 \%$ of the sample scored above the cut-score of 45 used to identify GAD on the Penn State Worry Questionnaire (PSWQ; Behar, Alcain, Zuellig, \& Borkovec, 2003; Meyer, Miller, Metzger, \& Borkovec, 1990), and 60\% scored above a cut-score of 65 used to distinguish GAD from other anxiety disorders (Fresco, Mennin, Heimberg, \& Turk, 2003). The PSWQ scores of the present GAD sample were comparable to PSWQ scores of people with GAD who present for treatment in clinical settings (Dugas et al., 2003, 2010; Ladouceur et al., 1999; van der Heiden et al., 2012; Wells et al., 2010), and the PSWQ scores of the non-psychiatric control group were equivalent to those reported by nonanxious groups in research (Startup \& Erikson, 2006).

The IUS scores of participants with GAD in the present study $(M=90.85, S D=17.93)$ appeared higher than those reported by individuals with GAD in randomized controlled trials of GAD treatment $(M s=68.49-83.36, S D s=16.54-24.50)$; (Dugas et al., 2003, 2010; Ladouceur et al., 1999; van der Heiden et al., 2012); however, the present IUS scores were comparable to those reported by individuals with GAD when a broader range of studies was considered (e.g., $M s=86.59-88.40, S D s=15.04-20.23$; Goldman, Dugas, Sexton, \& Gervais, 2007; Fracalanza et al., 2014; Khawaja \& McMahon, 2011). 
Table 2

Clinical Characteristics by Group $(N=40)$

\begin{tabular}{|c|c|c|c|c|c|}
\hline Questionnaire & $\begin{array}{c}\text { GAD } \\
(n=20)\end{array}$ & $\begin{array}{c}\text { NPC } \\
(n=20)\end{array}$ & $t$ & $\begin{array}{c}\text { Mann- } \\
\text { Whitney } U\end{array}$ & $\begin{array}{c}\text { Internal } \\
\text { consistency } \\
(\alpha)\end{array}$ \\
\hline IUS & 90.85 (17.93) & 42.25 (9.52) & $10.70 * *$ & $4.5 * *$ & .98 \\
\hline GAD-Q-IV- & $9.37(2.65)$ & $0.45(0.49)$ & $14.79 * *$ & $0.00 * *$ & .88 \\
\hline \multicolumn{6}{|l|}{ Continuous } \\
\hline PSWQ & $65.87(10.88)$ & $30.30(7.45)$ & $12.06 * *$ & $2.00 * *$ & .81 \\
\hline STICSA-T & 49.35 (12.27) & $25.70(5.40)$ & $7.99 * *$ & $17.50 * *$ & .95 \\
\hline STICSA-S & 38.75 (9.75) & $23.10(2.20)$ & $7.00 * *$ & $26.50 * *$ & .92 \\
\hline CES-D & 22.16 (9.25) & $4.92(4.11)$ & $7.62 * *$ & $24.00 * *$ & .76 \\
\hline
\end{tabular}

Note. GAD = Generalized anxiety disorder; NPC = Non-psychiatric control (i.e., no psychological disorder); IUS = Intolerance of Uncertainty Scale; GAD-Q-IV-Continuous = Generalized Anxiety Disorder Questionnaire - Continuously Scored; PSWQ = Penn State Worry Questionnaire; STICSA-T = State-Trait Inventory of Cognitive and Somatic Anxiety - Trait subscale; STICSA-S = State-Trait Inventory of Cognitive and Somatic Anxiety - State subscale; CES-D = Centre for Epidemiological Studies Depression Scale. Data were left-skewed on the GAD-Q-IV in the GAD group, and right-skewed on the GAD-Q-IV and CES-D in the NPC group; therefore the results of a nonparametric $t$-test equivalent (Mann-Whitney U test) which does not require the assumption of normality were presented alongside the traditional $t$-test.

$* p<.05, * * p<.01$ 
The IUS scores of the current study's non-psychiatric control group were comparable to the IUS scores of other nonanxious control groups (e.g., Holaway et al., 2006).

\section{Measures}

Eligibility assessment. The MINI (Sheehan et al., 1998) is a widely-used semistructured diagnostic interview that assesses for many DSM-IV Axis I disorders; note core DSM-IV GAD symptoms are unchanged in DSM-5 (Starcevic \& Portman, 2013). The MINI has very high interrater reliability ( $\kappa s=.88$ to 1.0 ), good test-retest reliability ( $r \mathrm{~s}=.76$ to .93 ), and high convergent validity with other clinical interviews (Lecrubier et al., 1997; Sheehan et al., 1998). The MINI Screen (Sheehan et al., 1998) can be used in conjunction with the MINI. It consists of 21 closed-ended gateway questions about current symptoms of Axis I disorders, and positive responses on the screen prompt administration of the corresponding module in the MINI. The MINI Screen has sensitivity ranging from 0.63 to 0.82 , and specificity ranging from 0.61 to 0.83 (Alexander, Haugland, Lin, Bertollo, \& McCorry, 2008).

\section{Qualitative interview.}

A semistructured interview guide was developed for the present study, which included four sets of questions designed to address the current study's research objectives. See page 38 for more information about how the interview guide was developed, and Appendix B for more details of the interview guide questions. A summary of key questions is presented below.

\section{Thoughts and underlying beliefs related to being uncertain.}

a) Thoughts when uncertain:

- "Please think of a specific uncertain situation that you were in recently, and responded in a way that was typical of you. What did you think to yourself in that situation?” 
b) Uncertainty as bothersome:

- "How do you feel about uncertainty?"

- (if participants said uncertainty can be negative) "What do you think it is about uncertainty, or being uncertain, that makes you feel that way?”

c) Personal meanings of being uncertain:

- “Does being uncertain ever mean anything about you personally?”

- "What does being uncertain mean about you personally?”

d) Uncertainty as unfair:

- "Can you give me an example of a time when it felt unfair to be uncertain, or unfair not to know things?”

- "What was it about that situation that seemed unfair?”

e) Assumptions about coping abilities and outcomes when uncertain:

- "When you're uncertain, do you tend to feel unsure about whether or not you will be able to cope with the outcome?”

- “When you don’t know how something will turn out, do you tend to assume it will turn out negatively?”

\section{Factors that may moderate attitudes toward possible negative outcomes. For each}

moderator of potential negative outcomes, participants were provided with at least one concrete example (see Appendix B for details), and responses were clarified through discussion.

b) Probability:

- "How do you feel in uncertain situations when you know there's only a small chance of something bad happening?” 
- "Do you react the same way when the chance of something bad happening is big or small?”

b) Cost:

- "How do you feel in uncertain situations where the consequences of something bad happening are minor?”

- "Do you feel the same way regardless of whether the consequences of something bad happening are minor or major?”

c) Others uncertain:

- "Do you feel differently about uncertainty when others are also uncertain, compared to when others have more information than you have?"

d) Controllability:

- "How do you feel in uncertain situations that are more in your control compared to uncertain situations that are less in your control?”

3. Attitudes toward positive and neutral uncertainty. Positive or neutral types of uncertainty may similarly be considered potential moderators of responses to uncertainty; however, there were additional questions asked about positive or neutral uncertainty, so these questions were presented as a separate section.

a) Uncertainty as positive:

- "How do you feel about uncertainty?"

- (if participants said uncertainty can be positive) "What do you think it is about uncertainty, or being uncertain, that makes you feel that way?”

- “Is uncertainty ever a good thing?” 
b) Responses to uncertainty about positive events:

- "How do you respond to uncertain situations that might be considered positive, or that are likely to turn out positively? For example, how do you react to not knowing what you will get as a gift?”

c) Uncertainty itself versus potential negative outcomes:

- "For you, is not knowing bothersome in itself, or is not knowing only bothersome when there's a potential negative outcome?” Responses were clarified through discussion.

\section{Systematically investigating clinical observations.}

a) Preference for certain negative outcome over uncertainty:

- "If someone is fighting a lot with their romantic partner and feeling uncertain about how their romantic relationship will turn out, some people say that they would prefer their romantic partner just leave them now, so that they will know with certainty what will happen, as opposed to remaining uncertain about the outcome. What do you think about this?”

Participants' preference for a certain negative outcome versus uncertainty was clarified through discussion. If participants mentioned that their preference was specific to relationships, then the interviewer asked them to consider their preference across different areas of life (e.g., work, finances) to arrive at their general preference. 
b) Allergy analogy:

- "If a person is allergic to something, they tend to have stronger responses than most people to even small amounts of the thing that they are allergic to. Some people have used the analogy 'allergic to uncertainty' to describe how they react to and feel about uncertainty. How does this idea relate to you?”

- “Do you feel like you are allergic to uncertainty?”

Questionnaires. Questionnaires assessing IU, GAD, anxiety, and depressive symptoms were administered for the purpose of describing the present sample and validating expected differences between the GAD and NPC groups.

The IUS (Buhr \& Dugas, 2002) is a 27-item measure of negative beliefs about uncertainty and its implications. The IUS has demonstrated excellent internal consistency $(\alpha=.95)$, good test-retest reliability, $(r=.74)$, and construct validity (Sexton \& Dugas, 2009).

The GAD-Q-IV (Newman et al., 2002) is a widely used self-report screening tool that assesses GAD symptoms and the degree of distress and impairment associated with these symptoms. The GAD-Q-IV can be scored using a continuous approach to create a score reflecting GAD severity, or a categorical approach in which individuals whose score above a threshold are said to meet GAD criteria (Moore et al., 2014; Newman et al., 2002). The GAD-QIV has shown convergent and discriminant validity, and good test-retest reliability (Newman et al., 2002).

The PSWQ (Meyer et al., 1990) is a 16-item self-report "gold standard" measure of the tendency to worry excessively. The PSWQ has high internal consistency ( $\alpha$ s $=.86$ to .91 ; Dear et al., 2011), and a range of test-retest reliabilities ( $r$ s= .74 to .92; see Startup \& Erickson, 2006 for a review). 
The State-Trait Inventory for Cognitive and Somatic Anxiety (STICSA; Ree, French, MacLeod, \& Locke, 2008) has two scales: 1) a 21-item Trait scale (STICSA-T) that assesses anxiety in general; and 2) a 21-item State scale (STICSA-S) that assesses anxiety in the present moment. The STICSA has demonstrated excellent internal consistency $(\alpha=.91$; Grös, Antony, Simms, \& McCabe, 2007) and good convergent and discriminant validity.

The Center for Epidemiological Studies-Depression Scale (CES-D; Radloff, 1977) is a 20item measure of depressive symptoms experienced over the past week. The CES-D has high internal consistency ( $\alpha=.85)$, adequate test-retest reliability $(r=.67)$, and has demonstrated concurrent and construct validity (Radloff, 1977).

\section{Qualitative Approach}

Sample determination. The present study used a purposive sampling method to recruit people with GAD $(n=20)$ and NPCs $(n=20)$. NPCs served as a disconfirming sample (i.e., control group), ensuring that the viewpoints of the GAD group were examined in the context of what is normative. A sample size of 40 was selected as this was comparable to qualitative or mixed methods studies in the anxiety disorders literature (e.g., Moore, Brødsgaard, \& Rosenberg, 2004; Marcus et al., 2011; Parrish \& Radomsky, 2010; Radomsky et al., 1998), and this sample size exceeds recommendations of how many interviews are typically required to achieve "saturation" (i.e., the point at which no additional themes arise in qualitative data; Guest, Bunce, \& Johnson, 2006; Kuzel, 1992).

Qualitative interview guide development. The first step in developing the semistructured interview guide involved conducting a thorough review of the IU literature to identify gaps in the current understanding of IU (see Introduction for a detailed rationale of each topic of interest). Once objectives for the semistructured interview guide were identified, this 
writer (KF) engaged in collaborative discussions with members of the research team (NK, KM, MA) in order to develop a list of potential questions that could address the study aims. Through discussion within the research team, the focus of the interview was narrowed. Next, this writer pilot tested the interview with a sample of individuals who met the present study's inclusion criteria $(n=4)$, and the interview was refined based on pilot testing (e.g., wording updated to be made more understandable).

An iterative approach was taken to the development of the semistructured interview guide, wherein updates were made to the interview after data collection commenced based on patterns of responses observed by the interviewer (KF). Using an iterative approach to interview guide development is consistent with best practices in qualitative research (Flick, 2009). After data collection commenced, this writer in collaboration with the research team decided to add several questions based on initial participant response patterns. These additions included specific prompts about assumptions when uncertain, additional questions about moderators of responses to uncertainty, and additional specific questions about uncertainty as positive or neutral.

Coding approach and codebook development. A thematic analysis was used to interpret the interview data, and was informed by the approach taken by Parrish and Radomsky (2010) to analyze qualitative data arising from semistructured interviews. Conducting a thematic analysis involves systematically identifying, analyzing and reporting patterns of responses (themes) within the data (Braun \& Clarke, 2006). A thematic analysis can be used when there is prior literature on a topic, as is the case with IU in people with GAD, and the goal of a study is to extend theory and research on a phenomenon that would benefit from further description (Braun 
\& Clarke, 2006; Hsieh \& Shannon, 2005). In the present study, participant phrases were the unit of analysis, and the meaning of phrases was examined in order to sort the phrases into themes.

The "sorting" of phrases into themes was accomplished using a document called a codebook, which defines themes to be coded in a dataset. Codebooks allow for participant responses to be organized into themes in a systematic, replicable manner (Flick, 2009). The present study used a two-phase approach to developing the themes that comprised the final codebook. First, given that there is an established literature on IU that allowed for some hypotheses to be made about how participants would respond to questions, an initial draft codebook was created prior to data collection that included these expected themes. Second, the draft codebook was updated throughout and following data collection based on participant responses, such that themes that were described by participants that were not present in the draft codebook were added to the final codebook (Crabtree \& Miller, 1999). This was accomplished through a processes whereby this writer (KF) and a research assistant (TM) independently immersed themselves in the data, reading and re-reading participant interview transcripts in order to identify any themes that were not included in the initial draft codebook that were present in the data. The researchers then discussed additional themes they had observed in the data to arrive at the final codebook (see Appendix C). The final thematic analysis presented in the results section was performed on all interview transcripts following the development of the final codebook. 
Reliability. All interviews were coded by the same primary rater (KF), and $50 \%$ of the sample was randomly selected and coded independently by a second rater to determine interrater reliability. The second rater was blind to participant group. The average interrater reliability of all research questions in the present study was 99.85\% agreement, with a Cohen’s Kappa ( $\kappa$ ) of .80. Cohen’s Kappa’s above .75 are considered excellent (Fleiss, Levin, \& Paik, 1981), as $\kappa$ is a conservative test that takes into account \% agreement and \% disagreement. See Table 3 for the $\%$ agreement and ks by interview question. Given the high interrater reliability, and the large number of themes in the present study, a decision was made to report the coding of the primary rater, as opposed to resolving each discrepancy through discussion.

\section{Procedure}

Prior to recruitment for the current study, pilot testing was conducted with a small sample $(n=4)$ meeting eligibility criteria, and the interview questions were refined based on pilot data. Interested individuals completed a telephone screen to determine eligibility, and those who met the eligibility criteria were invited to the Cognition and Psychopathology Lab at Ryerson University to participate. Participants signed a consent agreement (see Appendix D) prior to participation. During the lab visit, participants were interviewed individually, and the same interviewer (KF) conducted all interviews. Interviews were conducted individually so that discussions of potentially sensitive material would not be hampered by the presence of a group, and so that in-depth information from each participant could be gathered. All interviews were audio-recorded and later transcribed verbatim in a word processing program. All transcribed interviews were then uploaded to a software program called NVivo, which assists with the organization and analysis of qualitative data. Phrases were coded into themes based on the rules of the final codebook. 
Table 3

Interrater Reliability by Interview Question $(n=20)$

\begin{tabular}{lll}
\hline \multirow{2}{*}{ Interview Question } & Cohen’s & $\%$ \\
& Kappa $(\kappa)$ & Agreement \\
\hline
\end{tabular}

Objective 1. Thoughts and underlying beliefs related to being uncertain.
a) What thoughts do people have when uncertain?
.76
$99.87 \%$
b) Why is uncertainty bothersome?
.76
$99.85 \%$
c) What are the personal meanings of being uncertain?
.89
99.89\%
d) Why is uncertainty unfair?
.69
$99.68 \%$

Objective 2. Factors that may moderate attitudes toward possible negative outcomes
a) Probability
.84
99.85\%
a) Cost
.79
$99.72 \%$
c) Others also uncertain
.87
$99.81 \%$
d) Controllability
.85
$99.84 \%$

Objective 3.Exploring uncertainty as positive or neutral
a) Why is uncertainty positive?
.71
$99.84 \%$
b) How do you react to uncertainty about positive events?
.73
$99.88 \%$
c) Are you more bothered by uncertainty itself or possible negative outcomes?
$.89 \quad 99.92 \%$

Objective 4. Systematically investigating clinical observations
a) Would you prefer a certain negative outcome to remaining uncertain?
.82
$99.85 \%$
b) Do you feel allergic to uncertainty?
$.87 \quad 99.93 \%$

Note. Cohen’s Kappa’s above .75 are considered excellent, and between .40 and .75 are

considered fair to good (Fleiss, Levin, \& Paik, 1981). Cohen’s Kappa is a conservative test that takes into account \% agreement and \% disagreement. 
Coding was completed by each rater independently, with both raters blind to whether the interview was from a person in the GAD or NPC group. After coding was complete, the respective percentage of people in the GAD group and NPC group who endorsed each theme was calculated. These results were then entered into SPSS, and chi-square analyses were conducted on the proportion of people from each group who endorsed each theme. Examples of participant responses that were characteristic of each theme were extracted from interviews and are presented in the Results section. Following the interview, participants completed a demographics questionnaire (see Appendix E), the IUS, GAD-Q-IV, PSWQ, STICSA, and CESD. After participants completed all study components they were debriefed and compensated for their time (see Appendix F for debriefing form). 


\section{Results}

\section{Objective 1. Thoughts and Underlying Beliefs Related To Being Uncertain}

Table 4 shows the themes from responses to questions about thoughts and underlying beliefs about uncertainty that the GAD and NPC groups offered.

Thoughts when uncertain. Individuals reported on thoughts that they had in a recent uncertain situation where they responded in a way that was typical of them. The results showed that significantly more individuals with GAD (90\%) spontaneously reported thinking of negative outcomes when uncertain compared to $40 \%$ of people in the NPC group. For example, “I’ll obsess over the possibility of things not working out, like the worst case scenario” (GAD); “I'll feel like it’s just going to end badly” (GAD); “well I expect the worst” (NPC); and "so there were thoughts of fear, for the consequences” (NPC). In addition, a significantly higher proportion of individuals with GAD (80\%) spontaneously described having negative thoughts about themselves when uncertain compared to the NPC group (35\%). Examples of this theme were: “I still felt like I wasn’t good enough” (GAD); “I felt deficient” (GAD); “I could have performed better” (NPC); and “maybe I am not as good at this” (NPC).

There were also themes described only by people in the NPC group. Almost half (40\%) of the NPC group reported having positive thoughts about themselves when uncertain, such as "I felt confident in myself” and "I could handle uncertainty.” On the other hand, none of the GAD group described having positive thoughts about themselves when uncertain. In addition, $60 \%$ of the NPC group described thoughts of acceptance when uncertain, for example: "whatever happens, happens" and "it [uncertainty] is part of life so you just kind of have to deal with it." None of the GAD group reported thoughts consistent with the acceptance theme. 
Table 4

Thoughts, Beliefs, and Assumptions about Uncertainty $(N=40)$

\begin{tabular}{|c|c|c|c|}
\hline \multirow[b]{2}{*}{ Themes } & GAD & & \multirow[b]{2}{*}{$\chi^{2}$} \\
\hline & $(n=20)$ & $(n=20)$ & \\
\hline \multicolumn{4}{|l|}{ Thoughts in an uncertain situation $^{\mathrm{a}}$} \\
\hline Negative outcomes & $90 \%$ & $40 \%$ & $10.99 * *$ \\
\hline Positive outcomes & $20 \%$ & $35 \%$ & 1.13 \\
\hline Negative self-thoughts & $80 \%$ & $35 \%$ & $8.29 * *$ \\
\hline Positive self-thoughts & $0 \%$ & $40 \%$ & $10.00 * *$ \\
\hline Desire for certainty & $45 \%$ & $20 \%$ & 2.85 \\
\hline Unfairness & $30 \%$ & $10 \%$ & 2.50 \\
\hline Acceptance & $0 \%$ & $60 \%$ & $17.14^{* *}$ \\
\hline \multicolumn{4}{|l|}{ Reasons uncertainty is negative } \\
\hline $\begin{array}{l}\text { Because it interferes with being prepared or in } \\
\text { control }\end{array}$ & $85 \%$ & $35 \%$ & $10.42 * *$ \\
\hline $\begin{array}{l}\text { Because of the feelings and responses it causes me } \\
\text { (e.g., anxiety, muscle tension) }\end{array}$ & $80 \%$ & $15 \%$ & $16.94^{* *}$ \\
\hline Because I assume the worst case scenario & $60 \%$ & $0 \%$ & $17.14^{* *}$ \\
\hline Because it can resolve negatively & $20 \%$ & $40 \%$ & 1.91 \\
\hline When negative outcomes are likely or severe & $0 \%$ & $40 \%$ & $10.00 * *$ \\
\hline \multicolumn{4}{|l|}{ Personal meanings of uncertainty } \\
\hline Negative personal meaning ${ }^{\mathrm{b}}$ & $100 \%$ & $25 \%$ & $24.00 * *$ \\
\hline I’m incapable/incompetent & $65 \%$ & $10 \%$ & $12.91^{* *}$ \\
\hline I’m abnormal & $75 \%$ & $0 \%$ & $24.00 * *$ \\
\hline I’m unprepared & $35 \%$ & $20 \%$ & 1.13 \\
\hline Positive personal meaning & $0 \%$ & $20 \%$ & $4.44^{*}$ \\
\hline No personal meaning & $0 \%$ & $55 \%$ & $15.17 * *$ \\
\hline
\end{tabular}


Reasons uncertainty is unfair

I don't deserve to be uncertain

$\begin{array}{ccc}50 \% & 20 \% & 3.96 * \\ 60 \% & 0 \% & 17.14^{* *} \\ 70 \% & 65 \% & 0.11 \\ 10 \% & 30 \% & 2.50 \\ 10 \% & 25 \% & 1.56\end{array}$

Assumptions about coping abilities when uncertain ${ }^{\mathrm{c}}$

Can't cope

Can cope

Depends on situation

$\begin{array}{ccc}69 \% & 0 \% & 19.80 * * \\ 12 \% & 100 \% & 28.64 * * \\ 19 \% & 0 \% & 4.09 *\end{array}$

Assumptions about outcomes when uncertain ${ }^{\mathrm{c}}$

$\begin{array}{lccc}\text { Pessimistic } & 81 \% & 0 \% & 25.44^{* *} \\ \text { Optimistic } & 0 \% & 55 \% & 12.67 * * \\ \text { Realist } & 19 \% & 45 \% & 2.76\end{array}$

Note. GAD = Generalized anxiety disorder; NPC = Non-psychiatric control (i.e., no psychological disorder). Participants often offered more than one response, thus percentage totals do not add up to 100 . a Participants described uncertain situations where they responded in a way that they thought was typical of themselves; ${ }^{b}$ Percentage of individuals in each group describing at least one negative personal meaning of being uncertain; ${ }^{c} n=16$ in the GAD group as question was added after data collection had commenced.

$* p<.05, * * p<.01$. 
There were no differences in the number of people in each group who reported thinking about positive outcomes when uncertain. Examples of this theme included: "I was hoping for the best” (GAD) and "Whatever situation I'm in seems to turn out okay" (NPC). Similarly there were no between-group differences in spontaneous thoughts that the uncertain situation was unfair; for instance, “They [other people] have it all figured out and I'm so behind” (GAD) and "I thought it was unfair" (NPC). In addition, there were no significant quantitative differences in the number of people in each group who expressed thinking that they would like to be certain; however, there appeared to be a qualitative difference between groups. Individuals in the GAD group describing a more urgent desire for certainty (e.g., “I can’t be calm, I need to be certain”; "I need this resolved”) than did individuals in the NPC group (e.g., "I would always want to, more or less, know what’s in store”; “I like to know when things happen, I plan out my day”).

Reasons uncertainty is negative. Participants were asked about their attitude toward uncertainty, and if they indicated that uncertainty could be negative they were asked to provide reasons why. People said uncertainty could be negative because it interferes with being prepared or being in control, which was described by more people with GAD (85\%) than NPCs (35\%). Examples from the GAD group included: “If I can’t be prepared, so many things can go wrong”; “if I don’t have control over that kind of stuff it will be the death of me.” Examples from the NPC group included: "I want to be prepared”; "I like to have control over my life and when it is uncertain I don’t have any control.”

In addition, people said uncertainty was negative because of the feelings and responses it causes me; a theme present in the responses of significantly more people with GAD (80\%) than NPCs (15\%). Examples of this theme were: "Because uncertainty is at the root of a lot of my anxiety. So if there wasn't such thing as uncertainty, I wouldn't have so much anxiety.” (GAD); 
"it's that discomfort, the muscle tightening, the body tension but also the unfairness. So that's what makes the uncertainty so awful” (GAD); and "I don’t like my physical responses, it’s very uncomfortable, muscles tensing, and I find I overreact, I feel unhappy about that" (GAD); and "I think just the whole discomfort and anxious feeling that you get when under pressure” (NPC).

Another theme that emerged related to why uncertainty was negative was because I assume the worst case scenario; described by $60 \%$ of people with GAD and $0 \%$ of NPCs. Examples that were consistent with this theme, described only by those with GAD, included: "I am operating under this assumption that things are not going to go well for me. So that is the way that I react to it” and "because I think worst case scenario when I don’t know the outcome.”

People also said uncertainty was negative because it can resolve negatively. Note that this was different from the because I assume the worst case scenario theme, as responses consistent with the because it can resolve negatively theme did not reflect a general tendency to assume that all uncertain situations will resolve poorly, but rather concern that some uncertain situations can result in unfavourable outcomes. The because it can resolve negatively theme was described by people with GAD and NPCs. Examples of responses consistent with this theme included: "what's bad about that [uncertainty] is... say what happens in the end is a negative thing that you don’t want” (GAD); “the negative possibilities” (GAD); “I guess it would be the fear of something bad happening, I guess that would exist" (NPC); and "possible negative outcomes" (NPC).

Finally, individuals in the NPC group said that uncertainty was negative when negative outcomes are likely or severe; whereas none of the responses of the GAD group were consistent with this theme. Answers that illustrated this theme reported only by the NPC group included: "when there's really drastic consequences that rely on an uncertain decision"; "when the 
consequence is severe”; “if those [negative outcomes] are very strong and very likely”; and “it [uncertainty] is bad when the stakes are high.”

Personal meanings of uncertainty. Participants were asked about whether being uncertain ever means anything about them personally, and if so, what it means. All participants in the GAD group (100\%) said that being uncertain had one or more negative personal meaning, whereas significantly fewer (25\%) people in the NPC group reported that uncertainty had any personal meaning. Furthermore 20\% of NPCs said that being uncertain had positive personal meanings, and $55 \%$ of NPCs said that being uncertain had no personal meaning, themes that were not apparent in the responses of the GAD group.

The results showed that the negative personal meanings of being uncertain described by participants fell into three themes: I'm incapable/incompetent; I’m abnormal; and I'm unprepared. The I'm incapable/incompetent theme was described by significantly more people with GAD (65\%) than NPCs (10\%). Sample responses consistent with this theme included: "It [being uncertain] makes me feel useless...like I don't have any qualities to be able to fix the problem.” (GAD); “I am not capable enough.” (GAD); and "I must be incompetent” (NPC). Similarly, the theme I'm abnormal was reflected in the responses of significantly more people in the GAD (75\%) group compared to the NPC group (0\%), who did not describe responses consistent with this theme (0\%). Examples included: "I tend to think that I overreact in uncertain situations more so than normal people”; "there is something wrong with me"; and "I feel like I shouldn't be feeling the way I feel because I feel like other people don't.” On the other hand, the theme I'm unprepared was present in the responses of a comparable number of people in the GAD (35\%) and NPC (20\%) groups. Examples included: "I should have known that this could 
happen” (GAD); “I’m not prepared” (GAD); “it kind of means that you weren’t prepared enough" (NPC); and "I should have known how to do that" (NPC).

Examples of the positive personal meaning theme, described only by the NPC group included: "My personality ... the easy-going, adventure-seeking part really speaks to my positive outlook on uncertainty"; “It makes me feel like I'm spontaneous”; and "I’m confident in that I can make it for myself, I can make it ok.” Examples of the no personal meaning theme, described only by the NPC group included: “I don't think I think about myself when I'm uncertain”; "when I'm uncertain in the moment, I just feel like everyone else, I feel like it [being uncertain] happens to everyone”; and “no, I don’t think so [that uncertainty is personal].”

Reasons uncertainty is unfair. Participants were asked if they could provide an example of a time when uncertainty felt unfair; $90 \%$ of the GAD group and $75 \%$ of the NPC group indicated that uncertainty can be unfair and provided such examples. Significantly more individuals in the GAD (50\%) compared to NPC group (20\%) described that it was unfair to be uncertain in situations because they did not deserve to be uncertain. This theme reflected the idea that people did not do anything to warrant feeling uncertain, and yet the circumstances were such that they felt uncertain anyway. Responses consistent with this theme included: "In terms of my mother's situation [being uncertain due to mother's illness], I used to think it's unfair, like why do I have this problem? It's unfair that I have to deal with this.” (GAD); "For example with my girlfriend like I don’t know whether she is going to start a fight, and when it happens I feel that God is being unfair if that makes sense. It is like I didn’t do anything to deserve this.” (GAD); “I guess I just don’t feel like we [my partner and I] didn’t deserve it [to feel uncertain about living situation due to landlord]" (NPC); "I want to continue school, and the possibility of 
me not being able to because of something... I literally didn’t do anything wrong, but I needed a job.” (NPC).

Another reason for uncertainty being unfair commonly described by people with GAD (60\%) was that others cope better than me; and this theme was not present in the responses of NPCs (0\%). Sample responses from the GAD group were: “He [my partner] just doesn’t seem stressed...I get stressed out about things that he doesn’t.”; “It feels unfair that they [others] can be calm about it [uncertainty] and I can't be”; and “I'm going out with friends and they're like let's just see how it goes. And it just seems like...why do I have to feel so uncertain? Why can't I just go and enjoy? I just can't relax when I go out, until XYZ is known... Why is life so unfair that way?"

A high and comparable proportion of people in the GAD (70\%) and NPC (65\%) groups described uncertainty as unfair when another person was to blame. For example: "I respected their time by being ready, and they didn’t respect my time by letting me know that things have changed and they’d be late” (GAD); “when people won’t tell me why something is a certain way...it's just like why won’t you just tell me, so I can just know, so I can feel better?” (GAD); “if it was just like something being kept from me, but I feel like I should know...that would feel unfair” (NPC); and “I’m usually punctual and write back right away, so I expect the same treatment from others... when that doesn't happen it’s unfair.” (NPC).

Finally, a low and comparable proportion of people in the GAD (10\%) and NPC (30\%) groups described uncertainty as unfair when there is a very important or serious potential negative outcome. For example: “when he’s [husband] going in for surgery, it’s not fair that I don't know what's going to happen, because it’s such a big thing and I don't know the outcome, so to me that's not fair, because, it has such big implications.” (GAD); and "I felt like it was unfair 
because of the great impact it could have on my life. Losing my job would mean no income, and then I'd have to figure out how I was going to pay my tuition. So I felt like I needed to know" (NPC).

Assumptions about coping abilities and outcomes when uncertain. Individuals were asked whether they tend to assume that they can cope when they are uncertain. The results showed that almost $70 \%$ of people with GAD tend to assume that they cannot cope when they are uncertain, whereas $0 \%$ of non-psychiatric control participants described this. Sample responses from the GAD group included: “If it does end up negatively I don’t know how I am going to deal with that situation" and "Well every time, every single time there is that fear that I will not come out, that it is the end of the world, that I will not be able to solve it.” In contrast, sample responses from the NPC group included: “usually I think I'll be able to deal with whatever comes. In one way or another.” and "I feel like I have good supports in place and I'll be able to cope.”

Participants were also asked whether they tend to assume that uncertain situations will result in negative outcomes. The results showed that just over $80 \%$ of the GAD group said they tend to be pessimistic about the outcome when uncertain, for instance, "I always assume the negative" and "in uncertain situations I would rather think of the pessimistic side." On the other hand, $0 \%$ of the NPC group described assuming the worst when uncertain. In addition, significantly more people in the NPC group described no bias toward assuming negative or positive outcomes (45\%), compared to the GAD group (19\%). Examples of realist viewpoint were: "I try to think of all the possible outcomes, but I don't gravitate towards one unless it’s most likely" (NPC); "I don't have assumptions that are negative or positive” (NPC); and "I'd say I'm in the middle. More neutral.” (GAD). The results also showed that significantly more people 
in the NPC group (55\%) than GAD group (0\%) said that they tend to assume that uncertain situations would resolve positively. Sample responses that characterized this theme which was present in the NPC group only were: "I think I tend to be more on the positive side" and "I would say I'm often an optimist.”

\section{Objective 2. Factors That May Moderate Attitudes toward Possible Negative Outcomes}

Table 5 shows the themes that arose in response to questions about potential moderators of responses to possible negative outcomes in people with GAD and NPCs.

Probability. The results showed that significantly more people in the GAD group (50\%) compared to NPC group (10\%) said that the probability of a possible negative outcome would not change their attitude toward uncertainty. For instance: "even when the chance is low I still feel anxious" and "it [my response] tends to be the same because I think that $5 \%$ will beat me. Like if something is going to go bad, it's going to beat me. Even if it's a minute chance, I still feel it.” On the other hand, almost all non-psychiatric control participants (90\%) said that their attitude toward uncertainty is sensitive to how likely a potential negative outcome is, for example: "If there's a small chance of something bad happening, I would hardly worry about it" and "If it's a high probability I'll think about it more, it really does depend on that."

In addition to a quantitative difference between groups in terms of whether probability tends to be a moderator of their responses to uncertainty, there was also a qualitative difference in the group's answers. Of the 10 people with GAD who said that probability changes their attitude toward uncertainty, $80 \%$ reported that they would still worry even if probability was lower, whereas the NPC group indicated that they were not bothered by lower probabilities. For instance, people with GAD who indicated that probability moderated their attitude toward uncertainty said: "I would say I worry more if I think the chance is big, but it’s still a worry if it's small too" and "I do worry about it, but not as much as a bigger chance." 
Table 5

Factors That May Moderate Attitudes toward Potential Negative Outcomes

\begin{tabular}{|c|c|c|c|}
\hline Theme & $\begin{array}{c}\text { GAD } \\
(n=20)\end{array}$ & $\begin{array}{c}\text { NPC } \\
(n=20)\end{array}$ & $\chi^{2}$ \\
\hline Probability of a potential negative outcome & & & $7.62 * *$ \\
\hline Changes attitude & $50 \%$ & $90 \%$ & \\
\hline Does not change attitude & $50 \%$ & $10 \%$ & \\
\hline Severity of a potential negative outcome & & & $5.71 *$ \\
\hline Changes attitude & $75 \%$ & $100 \%$ & \\
\hline Does not change attitude & $25 \%$ & $0 \%$ & \\
\hline $\begin{array}{l}\text { Others also being uncertain about a potential negative outcome } \\
\text { (versus being the only one uncertain) }{ }^{a}\end{array}$ & & & 2.14 \\
\hline Changes attitude & $79 \%$ & $95 \%$ & \\
\hline Does not change attitude & $21 \%$ & $5 \%$ & \\
\hline Controllability of a potential negative outcome ${ }^{\text {b }}$ & & & 0.04 \\
\hline Changes attitude & $93 \%$ & $95 \%$ & \\
\hline Does not change attitude & $7 \%$ & $5 \%$ & \\
\hline
\end{tabular}

Note. GAD = Generalized anxiety disorder; NPC = Non-psychiatric control (i.e., no psychological disorder). ${ }^{\mathrm{a}} n=14$ in the GAD group; ${ }^{\mathrm{b}} n=15$ in the GAD group. Unequal $n s$ were due to questions being added after data collection had commenced.

$* p<.05, * * p<.01$ 
On the other hand, the NPC group did not indicate worry in response to a small chance of a negative outcome, for example, "usually I'm calm, fine with it [a small probability]."

Severity. The results showed that significantly more people in the GAD (25\%) compared to NPC (0\%) group said that the severity of a potential negative outcome does not change their response to uncertainty. Examples of responses suggesting that severity does not moderate attitudes toward uncertainty in the GAD group included: "that's something I need to work on because I still see it as, 'bad is bad.' If it is a huge negative consequence versus a little. To me a little is just as important. Especially if I can do something to possibly prevent it, it will.”; “I don’t like negativity anywhere. So you might describe something as minor, but I may think of it as major. So, any bad result could be a major thing. So, I would still take on the same steps I'd done before"; and "I still feel kind of the same... I still feel the same... I still tend to blow things out of proportion.”

There was also a qualitative difference between groups such that of the 15 people with GAD who said that severity changes their attitude toward uncertainty, approximately half (53\%) said that it does so only sometimes, or to a limited extent. For example: "depending on what it is" and "I would have to say it would change my discomfort, but only a little bit." To the contrary, of the 20 individuals in the NPC group who said that severity moderates their attitude toward uncertainty, most (80\%) said that it does so completely (i.e., not sometimes or partially). For example: "I think if the consequences were going to be big it would be much worse" and "well if the consequences are higher I worry about them more."

Others are also uncertain. A comparable proportion of people in the GAD and NPC group said that it is more tolerable to feel uncertain when others are also in the dark. Sample responses from the GAD group were: "when other people are uncertain I feel a lot better because 
I am not the only one. I will tend to think less negatively and more positively because they are also uncertain. But if they know more information than I do, then I feel excluded and left out” and "If everyone is in the same situation, I'd be like, 'everyone is feeling the same, I shouldn't care so much.' But if I'm in the category of people not knowing the outcome, I would feel more anxiety.” Sample responses from the NPC group were: "I think if we are all uncertain, I feel okay, we are all in the same boat. But if I am the only one uncertain and everyone else is certain then I am sure I would feel more anxious.” and “When I'm the only one who doesn't know the outcome I feel like something's being kept from me... and that bothers me more just on principle. But when everyone doesn't know what's going to happen, then I figure there's not really much that can be done about it.”

Controllability. The data showed that both the GAD and NPC groups indicated that the controllability of a potential negative outcome would moderate their attitude toward uncertainty, with more control making uncertainty more tolerable. Sample responses from the GAD group were: "the more control I have, the more I think I can minimize the possible negative consequences in the future” and "if I can control something, it’s probable easier to deal with...again, I can plan, I can do all that so that it will be, the possibility of it being a positive outcome is greater." Sample responses from the NPC group were: "most areas of uncertainty in my life don't bother me all that much, especially when I know that I have some control in the outcome" and "if I have some level of control, I have much more confidence in my own ability to achieve what I need to achieve, if it's completely out of my control then I start to get worried.” 


\section{Objective 3. Exploring Uncertainty as Positive or Neutral}

Table 6 shows the themes that emerged from questions about uncertainty with positive and neutral outcomes in people with GAD and NPCs.

Reasons uncertainty is positive. Most individuals in the GAD and NPC groups said that uncertainty can be positive (see Table 6); with no significant difference between groups. Individuals were asked why uncertainty can be a good thing, and both groups reported the same themes; there were no significant differences between groups in the proportion of people who discussed each theme, or in the manner in which themes were discussed, with the exception of the personal growth theme, which people with GAD described differently than NPCs.

People with GAD and non-psychiatric control participants said that uncertainty is positive when it leads to excitement, for example, "the exciting thing...the lottery ticket, the gifts, excitement during Christmas" (GAD) and "it is exciting! What are we going to do now? What ideas are we going to come up with next? It is just crazy business, so I guess uncertainty is our lifestyle.” (NPC). Along these lines, people also said uncertainty can be good when it supports a feeling of freedom and reduced rigidity. Sample responses were: "I might let someone else take the reins for a bit" (GAD) and "it is fun to just go with the flow basically" (NPC). People also described uncertainty as positive when it leads to positive outcomes. For example, “sometimes uncertainty is good, maybe it works out for the best, we don't always know” (GAD) and "when you don't know the outcome in a situation, you might end up having something positive...especially when all the possible outcomes are all positive things.” (NPC). 
Table 6

Exploring Uncertainty as Positive or Neutral

\begin{tabular}{|c|c|c|c|}
\hline Theme & $\begin{array}{c}\text { GAD } \\
(n=20)\end{array}$ & $\begin{array}{c}\text { NPC } \\
(n=20)\end{array}$ & $\chi^{2}$ \\
\hline Uncertainty can be positive & $90 \%$ & $100 \%$ & 2.11 \\
\hline \multicolumn{4}{|l|}{ Circumstances when uncertainty can be positive ${ }^{\text {a }}$} \\
\hline Excitement & $70 \%$ & $85 \%$ & 1.29 \\
\hline Feeling free (e.g., free of schedules, firm plans) & $25 \%$ & $35 \%$ & 0.48 \\
\hline Positive outcomes & $65 \%$ & $65 \%$ & - \\
\hline No/minimal negative outcomes & $30 \%$ & $20 \%$ & 0.53 \\
\hline Learning & $25 \%$ & $50 \%$ & 2.67 \\
\hline Personal growth & $40 \%$ & $45 \%$ & 0.10 \\
\hline Never positive & $10 \%$ & $0 \%$ & 2.11 \\
\hline Confusion at being asked about positive uncertainty & $45 \%$ & $0 \%$ & $11.61 * *$ \\
\hline \multicolumn{4}{|l|}{$\begin{array}{l}\text { How do you react to uncertainty that is considered positive } \\
\text { (e.g., not knowing what you will receive as a gift) a , b }\end{array}$} \\
\hline Positive Emotions & $84 \%$ & $100 \%$ & 3.26 \\
\hline Negative Emotions & $68 \%$ & $5 \%$ & $16.29 * *$ \\
\hline Negative Expectancies/Beliefs & $53 \%$ & $0 \%$ & $13.57 * *$ \\
\hline
\end{tabular}

Are you bothered by uncertainty itself or the possibility of negative outcomes? ${ }^{\mathrm{c}}$

$\begin{array}{lccc}\text { Uncertainty itself } & 29 \% & 0 \% & 6.49 * * \\ \text { Possible negative outcomes } & 18 \% & 95 \% & 21.94 * * \\ \text { Both } & 53 \% & 5 \% & 10.17 * *\end{array}$

Note. GAD = Generalized anxiety disorder; NPC = Non-psychiatric control (i.e., no psychological disorder).

${ }^{\mathrm{a}}$ participants often offered more than one response, thus percentage totals do not add up to 100 ; ${ }^{\mathrm{b}} n=$ 17 in the GAD group; $n=19$ in the NPC group; ${ }^{\mathrm{c}} n \mathrm{~s}=19$ in the GAD and NPC groups. Unequal $n s$ were due to questions being added after data collection had commenced.

${ }^{*} p<.05,{ }^{* *} p<.01$. 
Another circumstance in which individuals with GAD and NPCs categorized uncertainty as positive was when the potential negative outcomes were limited. Examples of responses included: “maybe I buy a lottery ticket...it’s uncertain...the only negative, you might lose a couple of dollars” (GAD) and “I guess just like little things, like when I’m at a local tournament and I don’t know who I'm going to be facing. It doesn’t really matter who I end up facing because normally it's somebody I've never played before [a positive outcome for this participant]” (NPC). Individuals in both groups also described uncertainty as positive when it leads to learning. For instance, "probably the chance to learn something new in a positive sense" (GAD) and “it [uncertainty] can be a good chance to learn something new and I think that's a good thing” (NPC).

Although both groups described uncertainty as positive under conditions where it leads to personal growth, there was a qualitative difference between each group’s responses. Individuals with GAD referenced their personal discomfort with uncertainty, and said that facing uncertainty despite discomfort can lead to personal growth, for example: "because of wanting to grow, although I am very uncomfortable with uncertainty I think in a roundabout way I can see it as a good thing" and "I feel like it can be [a good thing] because it is a challenge for me to deal with what it [uncertainty] feels like. I have to learn to deal with these situations where I might not be able to control the outcome or I don't know what is going to happen.” On the other hand, the NPC group described uncertainty as good when it leads to personal growth, but their responses did not reflect a personal struggle with uncertainty, but rather, a positive challenge for people in general. Sample responses in the NPC group were: "for people to stay challenged, stay reflective of themselves, because when you deal with uncertainty that's when you also draw on your inner, your inner feelings. I think that's when people learn more within themselves” and "I think just 
because it's so prevalent... uncertainty is real life. I think it just makes you more resilient and a stronger person having to go through that.”

Lastly, a small percentage of individuals with GAD noted that uncertainty is never a positive thing. No participants in the non-psychiatric control group said that uncertainty can never be positive. In addition, participants' initial responses to being asked if uncertainty can be positive were coded, as it was noticed by the interviewer (KF) that people with GAD seemed to react to the idea that uncertainty could be positive with surprise or confusion, and it was of interest to see if the difference in the initial responses of people with GAD and NPCs was statistically significant. There was a statistically significant difference in the proportion of people with GAD who responded with confusion to being asked about when uncertainty can be positive compared to the NPC group. Almost half (45\%) of the GAD group responded to this question by saying they were confused, or by requesting clarification and examples. On the other hand, $0 \%$ in the NPC group were confused when asked if uncertainty can be positive.

Responses to uncertainty with positive outcomes. Participants were asked how they react to uncertainty with positive outcomes (e.g., uncertainty about not knowing what one will receive as a gift). The results showed that both individuals with GAD and NPCs reacted to positive uncertainty with positive emotions. Examples of responses from people with GAD included: “That’s more enjoyable”; “Curious”; "I do feel happy for sure”; "It will put me in a good mood"; and "like an excited type of feeling." Responses from people in the NPC group were along the same lines: "always exciting”; "probably a bit of happy, a type of excitement”; "the uncertainty made it more interesting”; and "it’s kind of fun."

In addition, a difference emerged between groups showing that significantly more people with GAD (68\%) described negative emotions in response to positive uncertainty compared to 
NPCs (5\%). Examples of responses in the GAD group included: "I get myself prepared for disappointment”; “I do get nervous about receiving gifts”; "I get a little anxious”; "I probably get anxiousness just to know what's coming"; and "I would be worried about which one did I get and if that is the one I wanted."

A significantly greater proportion of individuals in the GAD group (53\%) also described negative expectations and thoughts about uncertain situations that typically are considered positive compared to the NPC group, who did not describe responses consistent with this theme (0\%). Examples of things that people with GAD said were: “I won’t like it”; “I definitely take the negative route and think 'that's great, but like, it's probably going to be this, or probably not going to be that much’ "; "I generally tend to believe that most uncertain situations will turn out negative”; and "I feel like I am thinking that I don’t want them to regret that they are giving me this gift... and that I wouldn't give them something back in return...so that's already something I am thinking about.”

Uncertainty itself versus possible negative outcomes. Individuals were asked if they were bothered by uncertainty itself, and not just the possibility of negative outcomes. The results showed that about one third of people with GAD (29\%) described being bothered by uncertainty itself, which was significantly more than the NPC group, who did not describe responses consistent with the uncertainty itself theme (0\%) (See Table 6). Examples of this theme were: “it’s just the feeling uncertainty” and “I feel like just not knowing is a negative thing.” About half of the GAD group (53\%) said that they were bothered by both uncertainty itself and possible negative outcomes, which was significantly more than the 5\% of NPCs who described responses consistent with this theme. Examples of responses that highlighted being bothered by both potential negative outcomes as well as not knowing in itself were: "It's kind of a mix of both, 
like, the outcome is definitely a part of it, but not knowing the outcome is just as much a part of it” (GAD); "I guess I'm bothered by not knowing more often than not, and bothered if there is a negative consequence that might happen” (GAD); and "They both resonate. I do not want to have any negative outcome and I do want it to be positive. On the other hand I do not like not knowing because that means that I cannot plan what is going to happen with me or what is going to happen with my life. That scares me. Not knowing to me...I almost equate that with negative.” (GAD). On the other hand, significantly more people in the NPC (95\%) compared to GAD (18\%) group reported being bothered by possible negative outcomes only. Sample responses consistent with this theme included: "Ya, not knowing if something will turn out bad" (NPC); “I think that what's bothersome is the possibility of negative outcomes. I don't think not knowing on its own is a bad thing" (NPC); and "That there could be a negative outcome" (GAD). 


\section{Objective 4. Systematically Investigating Clinical Observations}

Table 7 shows the themes reported by people with GAD and non-psychiatric control participants in response to questions testing clinical observations about IU that have been documented in treatment manuals and case descriptions of GAD.

Prefer a certain negative outcome versus uncertainty. The results showed that significantly more individuals with GAD (60\%) compared to NPCs (20\%) said they preferred a definite negative outcome to remaining uncertain. Examples of responses from people with GAD included: “I’d almost always rather just know, because then I could prepare for it”; “I would probably feel worse about not knowing”; and "I would prefer a concrete negative...so I can move on to different things.” In cases where NPCs described a preference for a certain negative outcome as opposed to tolerating uncertainty, responses were qualitatively similar to those provided by the GAD group. For example, people in the NPC group said: "I think I would go for the 'know already'...it allows you to know what to do" and "if you know that the outcome is negative, then you can plan.”

Compared to the GAD group (20\%), significantly more people in the NPC group (70\%) described preferring uncertainty to a sure negative outcome. Both groups who gave this response said that they preferred uncertainty because it allowed for the possibility of a positive outcome. For instance: "I would rather sit with uncertainty in the hope of figuring it out...of there being a positive outcome" (GAD) and "I'd rather have the uncertainty...if there was a chance of a positive outcome” (NPC). 
Table 7

Systematically Investigating Clinical Observations about Intolerance of Uncertainty

\begin{tabular}{lccc}
\hline Themes & GAD & NPC & $\chi^{2}$ \\
& $(n=20)$ & $(n=20)$ & \\
\hline
\end{tabular}

Would you prefer a certain negative outcome to being uncertain?

$\begin{array}{lccc}\text { Certain negative outcome } & 60 \% & 20 \% & 6.67 * * \\ \text { Being uncertain } & 20 \% & 70 \% & 10.10^{* *} \\ \text { It depends/unclear } & 20 \% & 10 \% & 0.78\end{array}$

Do you feel allergic to uncertainty?

$\begin{array}{lccc}\text { Yes } & 70 \% & 0 \% & 21.54 * * \\ \text { No } & 15 \% & 100 \% & 29.57 * * \\ \text { It depends/sometimes } & 15 \% & 0 \% & 3.24\end{array}$

Note. GAD = Generalized anxiety disorder; NPC = Non-psychiatric control (i.e., no psychological disorder).

${ }^{*} p<.05, * * p<.01$. 
Some people in the NPC group also referenced the fact that they were not bothered by uncertainty as part of their explanation for preferring being uncertain over a concrete negative outcome: “I’m ok with uncertainty, I wouldn't take the negative outcome”; “uncertainty doesn't scare me, I would rather the uncertainty"; whereas individuals in the GAD group did not describe being unaffected by uncertainty. A small and approximately equal proportion of people in each group said that their preference for a negative outcome over an uncertain one would depend on the circumstances.

Uncertainty allergy. Participants were asked whether they felt like they were "allergic" to uncertainty, as has been described in clinical writings (Dugas \& Robichaud, 2007). Most people with GAD (70\%) felt that this analogy fit their experience of uncertainty. People with GAD said: “I'm allergic to uncertainty...even if there is a little bit of uncertainty, I'd still react”; "Ya, I would say I have an allergy to uncertainty"; and "I do react... I get worried, I get nervous, sometimes I get sweaty and fidgety...I really am allergic to it.” On the other hand, no one in the NPC group said that the allergy analogy was consistent with their experience of uncertainty, for example: “No, I don’t mind uncertainty, I’m not allergic to it”; “Definitely not, no, it [uncertainty] is a part of life"; and "No, in fact, I want to have uncertainty to a certain extent, I think it’s healthy.” In some cases individuals with GAD (15\%) described being allergic to uncertainty in specific areas of life (e.g., “on a personal level I’m allergic to uncertainty”) or under specific circumstances (e.g., "if I'm in a happy mood the uncertainty might not affect me as much"). 


\section{Discussion}

The current study sought to uncover new insights about attitudes toward uncertainty in people with GAD compared to non-psychiatric control participants (NPCs) using a mixed qualitative and quantitative approach. The present study asked participants open-ended questions about: 1) thoughts and beliefs related to being uncertain; 2) factors that may change people’s responses to potential negative outcomes; 3) attitudes toward positive and neutral uncertainty; and 4) clinical observations about IU. The key ways in which the findings relate to previous research, extend IU theory, and inform GAD treatment are discussed below.

\section{Thoughts and Underlying Beliefs Related To Being Uncertain}

Typical thoughts when uncertain. There were several differences between people with GAD and non-psychiatric control participants in how they think when they are uncertain. To summarize: 1) significantly more people with GAD than non-psychiatric control participants reported thinking that an uncertain situation would resolve negatively; 2) a higher proportion of people with GAD versus non-psychiatric control participants described self-critical thoughts, such as doubts about their ability to manage an uncertain situation; 3) significantly more nonpsychiatric control participants than people with GAD reported positive self-thoughts when uncertain, such as reminding themselves of their positive characteristics or reasons they could get through the situation; and 4) significantly more non-psychiatric control participants than people with GAD reported thoughts reflecting acceptance of uncertainty. There were also several ways of thinking that were common to people with GAD and non-psychiatric control participants when uncertain. These included considering positive outcomes and describing thoughts related to unfairness. People with and without GAD also expressed thoughts reflecting a desire for certainty, although people with GAD expressed this desire using more strong qualitative terms than did non-psychiatric control participants. Since these findings overlap with those related to 
other research questions in the present study, they are explored in more detail later in the Discussion.

\section{Why is uncertainty negative?}

Uncertainty is negative because it interferes with being prepared or in control. The results showed that more people with GAD than non-psychiatric control participants said that they disliked uncertainty because it interferes with being prepared and in control. This is consistent with research showing that perceived lack of control is significantly and positively associated with anxiety and worry (Barlow, 2002; Başoğlu \& Mineka, 1992; Buhr \& Dugas, 2006; Foa et al., 1992; Rapee et al., 1996). This finding is also in line with theory suggesting that people with GAD engage in worry in an attempt to feel more prepared (Freeston et al., 1994; Holowka, Dugas, Francis, \& Laugesen, 2000) and in control of their concerns (Borkovec \& Roemer, 1995; Craske, 1999; Davey, 1994). Paradoxically, however, worry may actually prolong the experience of not feeling in control by increasing the amount of time a person spends considering uncontrollable stressors (Brosschot, Gerin, \& Thayer, 2006). In addition, the present finding that people with GAD believe uncertainty is aversive because it prevents preparation and control relates to findings on the behaviours of people with GAD. Specifically, individuals with GAD have been found to engage in more overpreparation and attempts to control their surroundings than non-psychiatric control participants (Study 1b of this dissertation; BeesdoBaum et al., 2012). It might be speculated that that employing such behaviours is an attempt to make uncertainty more tolerable.

Uncertainty is negative because of the feelings and responses it causes me. Most people with GAD reported that uncertainty is negative because of their own response to it, whereas a small proportion of non-psychiatric control participants described this theme. The 
result appears to relate to research suggesting that anxious individuals do indeed have more intense physiological and emotional responses to uncertainty than do nonanxious people (e.g., see Simmons et al., 2006, 2013). That most people with GAD and only a few non-psychiatric control participants find uncertainty aversive because of their own emotional responses to it may relate to Mennin et al.’s Emotion Dysregulation Model of GAD (Mennin, Turk, Heimberg, \& Carmin, 2004). This theory proposes that people with GAD have emotion regulation difficulties that contribute to and maintain pathological worry (Mennin et al., 2004). Among these difficulties is a tendency to experience negative emotions more strongly than nonanxious individuals as well as people with social anxiety disorder or depression (e.g., Decker, Turk, Hess, \& Murray, 2008; Mennin, Holaway, Fresco, Moore, \& Heimberg, 2007; Turk, Heimberg, Luterek, Mennin, \& Fresco, 2005). Another emotional difficulty of people with GAD according to Mennin et al. (2004) is that people with GAD have stronger negative responses to their own emotional experience compared to people with low anxiety and people with social anxiety disorder (e.g., Roemer, Salters, Raffa, \& Orsillo, 2005; Turk et al., 2005). These prior findings that people with GAD experience emotions more strongly than others, and react more negatively to their own emotions provide potential explanations for the present finding that the majority of individuals with GAD said that their own reactions to uncertainty were a reason why uncertainty is negative. It may be that people with GAD are bothered by their own reactions to uncertainty because they have difficulty accepting their emotional responses, or because their emotional responses are more intense than those of non-psychiatric control participants. This could be examined in future research to better understand the ways in which the IU Model of GAD (Dugas et al., 1998) and the Emotion Dysregulation Model of GAD (Mennin et al., 2004) may intersect. 
Uncertainty is negative because I assume the worst case scenario. Several findings in the present study pointed to the idea that people with GAD expect negative outcomes when uncertain. This is consistent with several previous studies showing that people with GAD tend to predict that future negative events will occur (MacLeod et al., 1991; Miranda \& Mennin, 2007; Provencher et al., 2000). These findings are also in agreement with research showing that people higher in IU interpret ambiguous scenarios as more threatening than do people with low IU (Dugas et al., 2005; Koerner \& Dugas, 2008). From a conceptual standpoint, it makes sense that the tendency to believe that uncertainty will result in a negative outcome would make a person more likely to be bothered by uncertain situations, as from this standpoint, not knowing is essentially equivalent to an unfavourable outcome.

Given that the tendency to assume the worst is a factor that makes uncertainty so aversive to people with GAD, clinical interventions aimed at challenging this stance may be useful. For example, the "uncertainty recognition and behavioural exposure” technique (uncertainty exposure) described in Dugas and Robichaud's (2007) manual for the cognitive behavioural treatment of GAD (CBT-IU) involves approaching an uncertain situation and recording thoughts that one had during and after the situation. A potential extension of this technique may be to ask individuals with GAD to articulate their predictions (e.g., the worst case scenario) prior to entering an uncertain situation, and then asking them to record what actually happened after the situation has resolved, with the goal of testing out the accuracy of the initial predictions (as per behavioural experiments; Bennett-Levy et al., 2004). Indeed, Hebert and colleagues (2014) have begun testing a variant of uncertainty exposure that involves asking people to first articulate their prediction about what will happen in a situation involving uncertainty, and then testing the prediction by engaging in the situation; that is, conducting a behavioural experiment testing 
one’s predictions about uncertainty. So far, results are promising, with authors finding that 12 weeks of a treatment focused on behavioural experiments that involve testing out negative

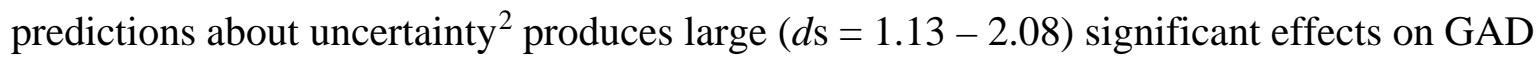
symptoms, anxiety, depression, and IU from pre- to posttreatment.

Uncertainty is negative because of the possibility of negative outcomes. A comparable proportion of people with GAD and non-psychiatric control participants said that uncertainty can be negative because it can result in negative outcomes. Responses consistent with this theme did not reflect a general tendency to assume that negative outcomes would occur when the person was uncertain. This result relates to the other present finding that almost all non-psychiatric control participants said that they were more bothered by uncertainty due to the possibility of negative outcomes than they were by uncertainty itself. On the other hand, the majority of people with GAD said that uncertainty was bothersome because of the possibility of negative outcomes as well as not knowing in itself. In sum, it seems that one of the main reasons why uncertainty can be negative from the perspective of non-psychiatric controls is because it can result in a bad outcome. On the other hand, for people with GAD, the idea that an uncertain scenario can unfold negatively is just one component of why uncertainty is experienced as negative.

Uncertainty is negative when negative outcomes are likely or severe. Almost half of non-psychiatric control participants said that uncertainty can be negative when negative outcomes are highly probable or severe. On the other hand, people with GAD did not mention the likelihood or cost of potential negative outcomes when they were asked why uncertainty is

\footnotetext{
${ }^{2}$ The treatment also included psychoeducation, uncertainty awareness training, and relapse prevention. On average participants received 9 sessions of behavioural experiments testing IU (Hebert et al., 2014).
} 
negative. That non-psychiatric control participants provided specific circumstances under which uncertainty could be negative as opposed to reasons why uncertainty was negative in general suggests that individuals without a psychological disorder may have a flexible attitude toward uncertainty that adapts depending on the situation in question. This present finding also relates to results on moderators of attitudes toward uncertainty; almost all non-psychiatric control participants said that they felt worse about potential negative outcomes that are more likely and severe, whereas responses in the GAD group about how probability and cost affect their attitude toward uncertainty were more mixed. Findings related to probability and cost of potential negative outcomes are described in more detail later in the Discussion.

Personal meanings of being uncertain. Everyone in the GAD group said that being uncertain meant one or more negative things about them, including that they were incompetent/ineffective, abnormal, or unprepared. In contrast, more than half of non-psychiatric control participants said that being uncertain was not personal, and a few people in the control group even said that being uncertain had positive personal meanings, for example, that they were spontaneous or confident. Of note, one of the reasons why individuals in the non-psychiatric control group said uncertainty was not personal reflected the notion that everyone feels uncertain, and it is normal to be unsure at times.

In terms of what the personal meanings of being uncertain are to people with GAD, responses to several questions in the present study converged on the notion that people with GAD believe that they are ineffective when uncertain. First, significantly more people with GAD than NPCs described being incapable or incompetent as a personal meaning of uncertainty. In addition, compared to individuals in the control group, more people with GAD spontaneously described negative beliefs about themselves when uncertain, including doubts about their ability 
to handle uncertain situations. Moreover, when asked directly if they tend to assume that they will not be able to cope when uncertain, this idea resonated with most people with GAD, but not with non-psychiatric control participants.

The findings in the present study suggesting that people with GAD believe they are incompetent/ineffective when uncertain are in line with theory and research suggesting that individuals with GAD may have underlying beliefs that reflect themes of personal inefficacy (Borkovec, 1994; Davey \& Levy, 1998; Hazlett-Stevens \& Craske, 2003; Koerner \& Dugas, 2008; Robichaud \& Dugas, 2005). Davey and Levy (1998) showed that high worriers were more likely than low worriers to describe their worries in a manner that reflected personal inadequacies and insecurities, and this was true even when the worry topic described by both high and low worriers was the same. Furthermore, people higher in trait worry show lower problem solving confidence (e.g., Davey, 1994, Davey, Hampton, Farrell, \& Davidson, 1992). In fact, low problem solving confidence and a negative attitude toward problems characterize individuals with high worry and correlate strongly with IU (Belzer, D'Zurilla, \& MaydeuOlivares, 2002; Fergus, Valentiner, Wu, \& McGrath, 2015; Robichaud \& Dugas, 2005). Moreover, although worriers demonstrate that they know the problem solving steps as well as nonworriers, negative beliefs about their own problem-solving abilities interfere with effectively executing problem solutions (e.g., Dugas, Letarte, Rheaume, Freeston, \& Ladouceur, 1995). In this way, beliefs about one's ineffectiveness when uncertain may become a self-fulfilling prophecy, in that a negative attitude about one's own abilities may actually prevent one from coping effectively under conditions of uncertainty. These experiences of feeling incompetent and unable to cope when uncertain may contribute to further intolerance of uncertainty (Koerner \& Dugas, 2008). 
In terms of helping people with GAD address the belief that being uncertain means that they are incompetent or ineffective, it seems that gaining experience coping effectively with uncertainty may be useful. It may be helpful for people with GAD to start approaching uncertain situations that are more manageable (e.g., trying an easy task with an uncertain outcome) versus a more overwhelming activity (e.g., trying a hard task with an uncertain outcome). Helping people with GAD to think systematically through all aspects of the problem-solving process and execute problem solutions may similarly help to break down beliefs about incompetence or ineffectiveness when uncertain. These suggestions are based on the strategies that comprise CBT-IU (Dugas \& Robichaud, 2007). It may be informative for future research to specifically measure how beliefs about competence change with various GAD treatment strategies.

In addition to beliefs about ineffectiveness and incompetence, the majority of people with GAD said that being uncertain meant that they were different from others in a negative way, and no individuals in the non-psychiatric control group described this theme. Thus, not only did people with GAD indicate that they are bothered by uncertainty because of their own emotional response to it, but it also seems that people with GAD believe that there is something wrong with their response, or that their response to uncertainty is atypical. The notion that people with GAD react more strongly to uncertainty has been proposed by clinicians who treat GAD (e.g., Dugas \& Robichaud, 2007) and by researchers examining the physiology of anxious individuals under uncertain circumstances (e.g., Grupe \& Nitche, 2013a). The present result highlights that people with GAD seem to be highly aware of their responses to uncertainty, thinking critically about such responses and analyzing how they compare to the responses of others. Further, the present study showed that people with GAD were able to articulate clearly their attitudes toward uncertainty. Being able to introspect and describe one's responses may be a strength of people 
with GAD, and this might be drawn on during assessment or treatment, for example, by asking people to identify their own nuanced beliefs about uncertainty.

Furthermore, it is of note that qualitatively, the way that people with GAD described the idea that they felt abnormal when uncertain appeared to be highly self-critical. For example, "I’m this anxious control freak that can’t let it go"; “I shouldn’t be feeling the way I feel”; "there’s something wrong with me”; "I feel crazy"; and "I have a personality or character shortcoming." The observation that people with GAD appear highly critical of their responses to uncertainty overlaps with another result from the present study - that people with GAD say that having negative thoughts about themselves is part of their typical response to uncertainty. The GAD group's tendency to think negatively about themselves when uncertain is in line with research showing a significant correlation between worry and self-blame (Meyer et al., 1990), and a study demonstrating higher levels of self-criticism in people with GAD than nonanxious controls (Dadds et al., 1993).

With respect to addressing the personal belief that one behaves abnormally when uncertain, it may be helpful for clinicians to provide additional psychoeducation about normative attitudes toward uncertainty in GAD treatment. Currently, the uncertainty exposure module of CBT-IU includes some psychoeducation about uncertainty. Namely, therapists provide information about the relationship between IU and worry, the inevitability of uncertainty in dayto-day life, and typical ways that individuals higher in IU tend to manage uncertainty. In addition to this, it may be helpful for people with GAD to know that although there are vast individual differences in preferences for certainty (Etner et al., 2010), it seems that on the whole, most people prefer certainty to uncertainty (Al-Najjar \& Weinstein, 2009; Epstein, 1999; Halevy, 2007). It may also be helpful for people with GAD to learn that attempting to be certain is 
adaptive and normative (e.g., Berger \& Calabrese, 1975; Brashers et al., 2000; Ellsberg, 1961; Kramer, 1999; Lipshitz \& Strauss, 1997), and that there is a likely evolutionary basis for most people feeling at least somewhat anxious when uncertain (Cosmides \& Tooby, 1996; Miceli \& Castelfranchi, 2005). Knowing more about normative attitudes toward uncertainty may help people with GAD reduce self-criticism about their own responses to uncertainty, and may facilitate greater acceptance of their emotions and experiences, potentially improving worry. A third negative personal meaning of being uncertain that both people with GAD and people in the control group described was that being uncertain meant that one is unprepared. Although this theme was discussed by both groups, it is possible that there was an undetected difference between groups in terms of how bad being unprepared is. It is hypothesized that being unprepared is more negative to people with GAD than it is to non-psychiatric control participants, based on the finding that more people with GAD say that uncertainty is negative because it prevents preparation/control, as well as research showing that individuals with GAD overprepare more than healthy controls (e.g., Beesdo-Baum et al., 2012). Another possibility is that believing that one is not prepared is part of a normative attitude toward being uncertain. In this case, beliefs that one is unprepared when uncertain might be expected to remain even after GAD is effectively treated.

One fourth of non-psychiatric control participants also described that being uncertain had a negative personal meaning, namely that they were unprepared or incompetent. Thus, it is not entirely atypical to have negative thoughts about the self when one is uncertain and, in fact, the negative beliefs about the uncertain self that people with GAD raised were the same beliefs that non-psychiatric control participants who had negative self-thoughts when uncertain described. A small proportion of individuals in the non-psychiatric control group fell on the other side of the 
spectrum, indicating that they had positive self-beliefs when they were uncertain, such as ideas that they were confident or spontaneous. The presence of both positive and negative thoughts about the uncertain self in non-psychiatric control participants indicates that the personal meaning of being uncertain may differ widely between people without a psychological disorder.

Having noted this, the majority of non-psychiatric control participants said that uncertainty is not personal. In their responses they shared ideas like "everyone is uncertain so it doesn’t mean anything about me when I am uncertain,” and “I do not think about myself when I am uncertain.” The tendency of individuals without a psychological disorder to believe that uncertainty is not personal may be one factor that helps them worry less than people with GAD in the face of uncertainty. Cognitive models suggest that there are patterns of negative thinking or "cognitive distortions" that tend to perpetuate negative emotions like anxiety (Beck et al., 1985; Ellis, 1962). One such cognitive error is called “personalizing,” which involves the assumption that the self is the cause of negative events or circumstances (Beck et al., 1985). The tendency to personalize has been associated with anxiety and worry (e.g., Beck et al., 1985;

Caudle et al., 2007). It may be that people with GAD feel more anxiety when uncertain because of the tendency to infer negative personal meanings (e.g., I'm uncertain because I'm unprepared or incompetent). On the other hand, non-psychiatric controls may remain less bothered by uncertainty because they tend not to think of uncertainty as personal.

Reasons uncertainty is unfair. A small proportion of people with and without GAD spontaneously described thinking that uncertainty was unfair when asked about their typical thoughts when uncertain, and most people, irrespective of their diagnostic status, related to the idea that uncertainty is unfair when asked to consider the idea directly. There were two reasons for uncertainty being unfair that were common to both groups. One was that it felt unfair when 
another person was to blame for them feeling uncertain (e.g., another person failed to provide information, creating feelings of uncertainty). Another was that it felt unfair to be uncertain about a situation with very serious potential negative outcomes (e.g., uncertainty about whether a loved one will survive an operation). These findings suggest that it is not exclusively a feature of GAD to believe that uncertainty is unfair in some circumstances.

There were also two reasons that uncertainty was perceived as unfair that were specific to the GAD group. The GAD group said that uncertainty was unfair because others are better at coping with uncertainty than they are. In other words, people with GAD find their own responses to uncertainty relative to the responses of others to be unfair, which seems to go hand in hand with the result that people with GAD are bothered by their own responses to uncertainty (e.g., worry, muscle tension). Naturally, it would feel unjust if one has an intense, uncomfortable response to uncertainty, whereas others seem unperturbed, and this notion also overlaps with the finding that people with GAD feel that they are "abnormal” when they are uncertain.

In addition, more people with GAD than non-psychiatric control participants discussed uncertainty as unfair in cases where they thought they did not deserve to be uncertain. Examples of this were being called on to speak in class when any student could have been selected, or suddenly needing to relocate due to circumstances beyond one's control. In other words, people with GAD said that it was unfair when they did not personally do anything to "bring on" the uncertain circumstance, and yet they found themselves affected by uncertainty anyway. This sense of unfairness may be related to the fact that people with GAD engage in more behaviours than do nonanxious controls to prevent feeling uncertain (Beesdo-Baum et al., 2012), and thus, may feel particularly cozened when uncertain events occur despite their best efforts to avoid this. 
The present findings coincide with research suggesting that beliefs about the unjust nature of uncertainty is a key aspect of IU. Indeed, the largest factor analysis of the IUS to date shows that it consists of just two underlying factors, one of which reflects the theme that “uncertainty is unfair and spoils everything” (IU-Factor-2; Sexton \& Dugas, 2009). It is well documented that individuals with GAD score higher on IU-Factor 2 than do healthy individuals (Birrell et al., 2011; Dugas et al., 2007; Khawaja \& Yu, 2010; Sexton \& Dugas, 2009), suggesting that individuals with GAD may have stronger or more diffuse beliefs surrounding the notion that uncertainty is unfair. The present finding that people with GAD discussed reasons why uncertainty is unfair over and above the reasons provided by people without a psychological disorder is consistent with these findings.

The current results also relate to recent research showing that anger is elevated in individuals with GAD (Deschênes et al., 2012; Erdem et al., 2008). It has also been found that IU helps to account for the association between GAD symptoms and anger (Fracalanza et al., 2014). Fracalanza et al. hypothesized that stronger beliefs that uncertainty is unfair in those with GAD may be one pathway that links GAD symptoms and anger. The present findings are consistent with this line of theorizing in that they indicate that people with GAD describe two additional reasons why uncertainty is unfair relative to non-psychiatric control participants. As such, it may be helpful in GAD treatment to examine and address beliefs related to uncertainty being unfair, as this may help to improve feelings of anger in people with GAD.

\section{Factors That May Moderate Attitudes toward Possible Negative Outcomes}

Probability and cost. Most people with GAD said that their attitude toward uncertainty was only slightly or not at all affected by the likelihood of possible negative outcomes; whereas non-psychiatric control participants said that their attitude toward uncertainty was definitely affected by the likelihood of possible negative outcomes. With respect to cost, one quarter of 
people with GAD said they would feel equally bothered by uncertainty regardless of the severity of the potential negative consequences. No individuals in the non-psychiatric control group said their attitude toward uncertainty would be unaffected by the severity of the potential consequences. Of people with GAD who said that the severity of outcomes would change their attitude toward uncertainty, about half said that it would do so only to a small extent. Thus, overall, the GAD group appeared split in terms of whether cost moderated their attitudes toward uncertainty; whereas the non-psychiatric control group’s attitudes toward uncertainty were very clearly impacted by the size of the negative consequences.

These findings inform theory and clinical observations suggesting that people with GAD respond negatively to uncertainty regardless of the probability or cost of the uncertain event (e.g., Dugas et al., 2004; Dugas \& Robichaud, 2007; Miceli \& Castelfranchi, 2005). Indeed, an early definition of IU stated that IU is a "predisposition to react negatively to an uncertain event or situation, independent of its probability of occurrence and of its associated consequences.” (Ladouceur et al., 2000, p. 934). The present findings suggest that these descriptions may be partially true in that most people with GAD are at least somewhat bothered by uncertainty even when the probability of feared events is low, and some people with GAD are at least somewhat bothered by uncertainty even when the potential consequences are minor. However, the current data suggest that attitudes toward uncertainty in people with GAD may not be entirely independent of the associated probability and cost of potential negative outcomes.

The results suggest that more people with GAD are insensitive to the probability versus the severity of potential negative outcomes. Theories suggest that perceptions of the likelihood and cost of a potential negative outcome, as well as perceptions of coping abilities influence a person's overall response to a perceived threat (e.g., Borkovec et al., 2004; Smith \& Lazarus, 
1993). Some people with GAD may show less negative attitudes toward uncertainty when potential negative outcomes are minor because smaller consequences may be thought of as easier to cope with. Indeed, one of the other results from the current study was that people with GAD tend to assume that they will not be able to cope when they are uncertain, and it is possible that this belief is modified by the severity of negative outcomes, although people were not asked about this in the present study specifically. On the other hand, any probability of a nonminor potential negative outcome may elicit a negative attitude toward uncertainty from people with GAD due to beliefs about one's inability to cope with a medium sized, or worse, "major" negative outcome. In sum, it is supposed that beliefs about being unable to cope may lead people with GAD to have a negative attitude toward even very unlikely potential negative outcomes, whereas some people with GAD may believe that they can cope with uncertainty when potential negative outcomes are known to be small.

Non-psychiatric control participants tended to report being unbothered by uncertainty with low likelihood negative outcomes or minor consequences. This is in line with prior research showing that individuals who believe that negative outcomes are less likely tend to worry less than people who believe that negative outcomes are more likely (Berenbaum, Thompson, \& Bredemeier, 2007; Berenbaum, Thompson, \& Pomerantz, 2007; Butler \& Mathews, 1983). Indeed, improving perceptions of the probability and cost of threatening events is theorized to be a key underlying mechanism of change in CBT for anxiety disorders (e.g., Beck et al., 1985; Clark, 1999). Several studies have tested this theory in people with social anxiety disorder (SAD), with results showing that improved perceptions of the probability and cost of threatening events are associated with improved anxiety in people with SAD (Hoffart, Borge, Sexton, \& Clark, 2009; Nelson, Deacon, Lickel, \& Sy, 2010; Taylor \& Alden, 2008). 
The present findings raise the possibility that there may be differences in how people with GAD and people with other anxiety disorders such as SAD respond to probability and cost information. This question would be of interest to study given that IU is equally elevated across anxiety disorders (e.g., Carleton et al., 2012; Mahoney \& McEvoy, 2012), and it is not yet known if attitudes toward uncertain situations are moderated by the same factors in different anxiety disorder populations.

A cognitive strategy in CBT involves challenging the probability and cost of potential negative outcomes (see Brown, O’Leary, \& Barlow, 2001; Borkovec \& Costello, 1993; Rygh \& Sanderson, 2004; Zinbarg, Craske, \& Barlow, 2006). GAD treatments that include strategies aimed at challenging probability and cost biases have been found to have medium to large significant effects on GAD symptoms when the whole treatment package is evaluated (e.g., Forsyth \& McNeil, 2002). However, it is unclear if traditional cognitive restructuring (i.e., challenging perceptions of the probability and cost of feared outcomes) contributes to these effects, as the degree to which challenging probability and cost biases improves worry has not been tested specifically. Further, GAD-specific treatments that do not include techniques to challenge perceptions of the probability or cost of feared outcomes have shown large significant effects on worry and associated symptoms (e.g., Dugas et al., 2010; Wells et al., 2010).

It is also possible that techniques focused on re-evaluating the probability of feared events may inadvertently maintain intolerance of uncertainty and worry by providing a sense of reassurance that feared events are remote or unlikely, potentially precluding opportunities to "get used to” feeling uncertain without doing anything to control feelings of uncertainty. Reestimating probabilities may even be viewed as somewhat antithetical to "rolling with" uncertainty (Dugas \& Robichaud, 2007; Koerner \& Dugas, 2008). Further, strategies that 
minimize the probability of feared events may be less practical in the long-term than strategies emphasizing acceptance of uncertainty, since new uncertain situations will continuously arise, leaving an infinite number of potential threats to challenge. Thus, current treatment guidelines for improving IU in people with GAD recommend strategies aimed at increasing tolerance for uncertainty over approaches aimed at reducing perceived uncertainty (Carleton, 2012; Dugas \& Robichaud, 2007; Robichaud, 2013).

Unknowable versus unknown uncertainty. The results showed that most participants in the GAD and NPC groups described "unknowable uncertainty” (i.e., when everyone is uncertain) as more tolerable than "unknown uncertainty" (i.e., when they are the only person uncertain). Although there was no significant difference between the GAD versus nonpsychiatric control group in terms of whether unknowable versus unknown uncertainty was a moderator of their attitude toward uncertainty, nonetheless, a small proportion of the GAD group (21\%) said that their attitude toward uncertainty would remain unchanged regardless of who else was uncertain. This nonsignificant finding is in line with the present results on probability and cost as moderators of attitudes toward uncertainty, reflecting the notion that compared to individuals without a psychological disorder, fewer people with GAD adapt their responses to uncertainty based on contextual factors, which would theoretically make people with GAD more vulnerable to worry in a variety of uncertain situations.

The finding that non-psychiatric control participants described uncertainty as less bothersome when others are also uncertain versus when they are the only one uncertain replicates Chow and Sarin's (2002) experiments showing that individuals in a nonclinical sample prefer not knowing when "everyone is in the dark" compared to when they are the only one uncertain. The present study also suggests that unknowable uncertainty is more tolerable to most people with 
GAD than unknown uncertainty, implying that even those who are highly intolerant of uncertainty find it easier to be uncertain when others are uncertain alongside them. This finding aligns with the comparative ignorance hypothesis proposed by Fox and Tversky (1995), suggesting that people prefer situations where they have at least as much information as others to situations where they have less information. The tendency to feel better when one is not alone in being uncertain may be influenced by the human tendency to prefer fairness, or equally distributed resources (see Brockner \& Wiesenfeld, 1996); in this case, the idea that one should have access to the information if it is available to some. The results may also be influenced by the human need to be included in a group (e.g., Baumeister \& Leary, 1995). In line with this guess, some people in the present study described that they would feel "left out" if other people had information that they did not have. In sum, it seems typical of people with and without GAD to feel that "missing information is more palatable when it is unavailable to all” (p.127; Chow \& Sarin, 2002).

Controllable versus uncontrollable uncertainty. Almost all individuals with and without GAD described a preference for uncertain situations that allow for more control over the outcome to uncertain situations that offer less control. These findings are in agreement with those of Zvolensky and colleagues (2001), who found that in a nonclinical sample, lower perceptions of control over one's anxiety increased the degree to which people perceived ambiguous situations as threatening. The finding that people with GAD were more bothered by uncertain situations which offered less control is also consistent with another current finding showing that one reason why people with GAD believe uncertainty is negative is because it interferes with being prepared and in control. Taken together, these findings suggest that the perception of control moderates how most people with GAD respond to not knowing. 
It is also of note that there were no differences in the proportion of people in the GAD compared to NPC group who said that their degree of control over an uncertain situation changed how they felt about it, suggesting that it is normative to feel more anxious and bothered by uncertain situation where one has less versus more control over the outcomes. These findings are in keeping with a large body of research demonstrating a robust link between perceived lack of control over a threat and higher anxiety in nonanxious individuals (e.g., Breier et al., 1987; Geer, Davison, \& Gatchel, 1970; Peters et al., 1998) as well as individuals with anxiety disorders (e.g., Barlow, 2002; Başoğlu \& Mineka, 1992; Foa et al., 1992; Mineka \& Zinbarg, 1996). Indeed, low control over a stressor is thought to be sufficient to produce "generalized fear and arousal” in humans (Miceli \& Castelfranchi, 2005), and furthermore, recurrent uncontrollable stressors are understood to lead to the development of anxiety disorders (e.g., Mineka, 1985). Thus, overall, perceived degree of control over whether bad things will happen has long been found to have implications for anxiety, and the present study suggests that greater control over uncertain situations is no exception to people with GAD or non-psychiatric controls.

Overall, the findings suggest that the probability of a potential negative outcome does not have a large impact on attitudes toward uncertainty in most people with GAD, and the severity of a potential negative outcome affects attitudes toward uncertainty to some degree in some people with GAD. Further, others also being uncertain changes attitudes toward uncertainty in the majority of people with GAD, and the controllability of an outcome impacts attitudes toward uncertainty in almost all individuals with GAD. On the other hand, probability, cost, others also being uncertain, and controllability impact attitudes toward uncertainty in most non-psychiatric control participants. These findings suggest that not all forms of uncertainty are equally intolerable to people with GAD and that uncertainty is not a unitary construct. Implications of 
the idea that there are different "types" of uncertainty will be explored further in the General Discussion of this dissertation.

\section{Exploring Uncertainty as Positive or Neutral}

Reasons uncertainty is positive. There were no differences between individuals with GAD and non-psychiatric control participants in terms of their descriptions of the positive aspects of uncertainty. The most commonly described reasons why uncertainty is positive reflected themes of excitement, positive outcomes, personal growth, and learning. The themes found in the present study are consistent with research on what is positive about uncertainty. A large body of previous studies have shown that uncertain scenarios can be exciting and interesting (e.g., Bar-Anan et al., 2009; Lee \& Qiu, 2009; Loewenstein, 1994; Norton, Frost, \& Ariely, 2007; Wilson, Centerbar, Kermer, \& Gilbert, 2005). For example, the marketing literature suggests that uncertainty about discounts and promotions (e.g., "scratch and save" promotions) foster greater interest and excitement in consumers, and stimulate more purchases (Dhar, Gonzalez-Vallejo, \& Soman, 1999; Goldsmith \& Amir, 2010; Grant \& Tybout, 2008). In line with this, individuals generally like to view live as opposed to previously taped events on television because the outcomes are unknown and inherently exciting (Vosgerau, Wertenbroch, \& Carmon, 2006).

Further, the results that learning and personal growth are positive aspects of uncertainty are consistent with research suggesting that uncertainty facilitates learning. People may reduce feelings of uncertainty by considering different ways that the situation could resolve, and as

such, people may think about uncertain information more deeply than they do about absolute facts (Tormala et al., 2012). Furthermore, when people have experiences that run counter to their initial expectations, resolving such expectancy violations may promote new learning (Rescorla \& Wagner, 1972). Research has shown that people recall more information about 
messages with content that is incongruent versus congruent with an original message, presumably because people have to think about the inconsistency to make sense of it (Maheswaran, \& Chaiken, 1991).

Being uncertain may provide opportunities for learning and personal growth, in that initially a person may not know how to make sense of an uncertain situation, and then may learn through having to "work through it.” However, people with GAD may not fully take advantage of the learning and personal growth that can occur in uncertain situations due to the use of behaviours that minimize feelings of uncertainty (Beesdo-Baum et al., 2012; Study 1b of this dissertation). These behaviours include seeking advice from others about how to navigate the situation, looking up a lot of information before moving forward, and even avoiding uncertain situations altogether when possible. The use of behaviours that reduce and avoid uncertainty may prevent opportunities for learning and personal growth, potentially serving to maintain IU. Overall, the present study replicates findings on reasons why uncertainty is positive that have been suggested in past studies, and contributes the novel result that people with GAD perceive uncertainty to be positive for the same reasons as non-psychiatric control participants.

In treating GAD, it may be helpful for therapists to ask clients about the reasons why uncertainty can be positive, particularly if the treatment will involve honing greater acceptance of uncertainty (e.g., CBT-IU). Aspects of uncertainty that can be positive, as uncovered in the present study, could be used as starting points for a conversation on the benefits of uncertainty. Considering the positive side of uncertainty may facilitate a more balanced perspective in individuals with GAD, and may increase people's willingness to approach uncertain situations. In addition, it is of note that although the present study did not find differences between people with and without GAD in terms of the circumstances under which uncertainty can be positive, it 
is possible that the degree to which uncertainty is positive (mildly versus extremely positive) may differ between people with GAD and nonanxious controls. This could be explored in future research.

Attitudes toward uncertainty about positive outcomes. People with GAD and nonpsychiatric control participants were asked to describe how they feel in response to not knowing what they will get as a gift, and both groups described feeling positive emotions in this situation. However, people with GAD also said that they would feel negative emotions about getting a gift, and described negative expectations about how the situation would resolve, whereas NPCs did not offer these kinds of responses. These results converge with another finding in the present study that although both the GAD and NPC groups reported thinking about positive outcomes in an uncertain situation, significantly more people with GAD than NPCs also discussed thoughts of negative outcomes. Along these lines, research has shown that people with GAD are similar to people with depression in that both tend to predict that negative events will occur; however, people with GAD do not predict an absence of positive events, whereas people with depression do (Miranda \& Mennin, 2007).

The present finding that people with GAD report negative emotions and expectancies in the context of a situation without threatening outcomes is also in line with work on the nature of pathological worry. Davey and Levy (1998) found that high worriers were more likely than low worriers to "catastrophize” (i.e., express concerns about) a positive aspect of their life. Indeed other research has similarly shown that high worriers identify more negative features in life events that are generally considered neutral or positive than do nonworriers (Davey et al., 1992). Thus, overall it seems that people with GAD are more prone than people without a psychological 
disorder to experiencing negative emotions and expectations when uncertain, even when all outcomes are positive or neutral.

Uncertainty itself is bothersome. Most people with GAD said that they were bothered by uncertainty itself, even in the absence of negative outcomes. If not knowing in and of itself is threatening to people with GAD, then it makes sense that not knowing what one will receive as a gift would induce negative emotions and expectations. The finding that people with GAD find not knowing in the absence of possible negative outcomes aversive is consistent with theorizing emphasizing a fundamental fear of the unknown in GAD, even when negative outcomes are nonexistent or extremely unlikely (Dugas et al., 1995; Dugas, Hedayati, et al., 2005; Freeston et al., 1994).

The finding that people with GAD seem to respond more negatively than do others to uncertainty itself is consistent with a set of experiments by Kupor and colleagues (2014). They showed that most individuals in a nonclinical sample reported higher interest and desire to try something when a positive idea was framed in uncertain terms (e.g., "she may be the best chef in town”) than they did when a positive idea was framed in absolute terms (e.g., "she is definitely the best chef in town”). However, in Kupor et al.'s study, nonclinical community participants higher in IU did not show the same preference for the framing of positive events in uncertain terms that other participants showed. Rather, people higher in IU showed comparable desire to try and interest in a positive event framed in uncertain versus absolute terms in one experiment, and comparable desire to try but lower interest in a positive event framed in uncertain versus absolute terms in another experiment. This suggests that people higher in IU may not respond to “positive” uncertainty in the same way as people lower in IU, and may interpret uncertainty in a less positive manner than do others, even in the absence of threatening outcomes. 
In addition, it is of note that the majority of people with GAD described being bothered by potential negative events in addition to being bothered by uncertainty itself. This is in line with research in a nonclinical student sample showing that the relationship between a person's level of IU and their level of worry about a scenario was stronger for ambiguous scenarios with potential negative outcomes as well as for ambiguous scenarios with only positive possible outcomes compared to scenarios with definite outcomes (Byrne et al., 2015). Further, Byrne et al. provided initial statistical evidence that there may be two pathways that connect higher IU and worry. Byrne and colleagues showed that the relationship between IU and worry in ambiguous scenarios with potential negative outcomes is partially accounted for by perceptions of the probability and cost of negative outcomes. This suggests that one "pathway" from high IU to worry is through an attentional bias toward perceiving negative outcomes as likely and costly (Koerner \& Dugas, 2008). The results of Bryne et al.’s study also showed that the association between IU and worry in ambiguous scenarios without potential negative outcomes (i.e., several potential positive interpretations) was largely not accounted for by perceptions of the probability and cost of negative outcomes. This suggests that there may also be another "pathway" from high IU to worry, which Byrne and colleagues said may involve the "need to anticipate" and anxiety when one is unable to anticipate an outcome. The present findings support Byrne et al.'s idea that there may be two pathways through which uncertainty is associated with worry, by providing preliminary evidence from a GAD sample that both uncertainty with potential negative outcomes and uncertainty itself seem to produce concern.

In the current study, non-psychiatric control participants indicated that they were more bothered by not knowing whether potential negative events would occur than they were by not knowing in itself. However, it is of note that uncertainty without negative outcomes may still 
produce discomfort in healthy people. Research on healthy humans and rodents has shown that unpredictably timed neutral tones elicited greater amygdala responding and anxious behaviour than did predictably timed neutral tones (Herry et al., 2007). It has also been shown that compared to non-psychiatric control participants, people with either panic disorder or posttraumatic stress disorder display greater startle in response to an unpredictable versus predictable neutral stimulus (Grillion et al., 2008, 2009). Thus, although the present study found that negative uncertainty is more concerning than neutral uncertainty to non-psychiatric control participants, prior research suggests that neutral uncertainty may still provoke a small degree of anxiety in most people.

\section{Systematically Investigating Clinical Observations}

Preference for a negative outcome over an uncertain outcome in GAD. Consistent with clinical observations, the current results showed that significantly more people in the GAD compared to non-psychiatric control group said that they would prefer a negative outcome that is certain to tolerating uncertainty because it prevents the painful feelings that go along with being uncertain, and allows for planning about what to do next. There was also the idea that knowing the outcome with certainty would save time and effort preparing for many possible outcomes, and therefore knowing, even if negative, was efficient. On the other side of the coin, significantly more non-psychiatric control participants than people with GAD said that they would prefer to tolerate being uncertain than accept a certain negative outcome. The main rationale that people provided reflected the notion that sitting with uncertainty allowed for the possibility that events could resolve favourably - a chance for a positive outcome. People without a psychological disorder may have considered the possibility of positive outcomes because they had an easier time bringing to mind ideas about uncertainty resolving in a positive manner relative to people with GAD. This is suspected because people show biases in the 
encoding and recall of mood-congruent information; individuals in a positive mood have an easier time retrieving ideas about happy outcomes (see Beck et al., 1985; Bower, 1981; Coles, Turk, \& Heimberg, 2007; Matt, Vázquez, \& Campbell, 1992). A small proportion of people in each group said that their preference for a sure negative outcome to remaining uncertain would depend on the circumstances.

There is some evidence that even non-psychiatric control participants prefer a sure negative outcome to an uncertain outcome in some cases (Lieberman, Cathro, Nichol, \& Watson, 1997), such as when a negative outcome is minor or when being certain is important (Lieberman et al., 1997). Indeed, a nonclinical sample showed a preference for pressing a button that indicated that they would definitely not be rewarded to a button that provided no information about future rewards (Lieberman et al., 1997). To interpret this, it was suggested that having information, even if negative, can be reinforcing in and of itself (Hendry, 1969; Perone \& Kaminsky, 1992). Further, the more valuable the information, the more reinforcing knowing it may be (Berlyne, 1957; Lieberman et al., 1997). This may explain why more people with GAD in the present study showed a bias toward accepting a concrete negative outcome over being uncertain. It might be hypothesized that having information is particularly reinforcing to people with GAD, as knowing may prevent perseverative thinking and uncomfortable feelings. It may be that people with GAD value bad news over no news because bad news signals relief from the worry process, which presumably would be quite valuable. Moreover, knowing an outcome, even if negative, provides people with the opportunity to move on (versus continuing to wonder what will happen) and to problem solve.

The GAD group’s general preference for a negative outcome over being uncertain may have conceptual links to the present study's other finding that people with GAD tend to assume 
the worst case scenario when uncertain. In other words, one way that people with GAD may cope with uncertainty may be by giving up on the idea that things may resolve positively, and instead, fully believing that a negative outcome is bound to ensue, which is sometimes referred to as “pessimistic certainty” (Miranda \& Mennin, 2007). Indeed, in his review of the literature Carleton (2012) theorized that higher IU may make people prone to pessimistic certainty and that “accepting negative consequences as inevitable may be preferred to tolerating uncertainty” (p. 5). In line with this idea, Miceli and Castelfranchi (2005) suggested that assuming a concrete negative outcome may provide benefits to anxious individuals because considering one outcome is more controllable and predictable than considering indefinite possible outcomes.

The present finding also bears theoretical similarity to the main tenet of the Contrast Avoidance Theory of GAD (Newman \& Llera, 2011; Newman, Llera, Erickson, Przeworski, 2013), for which there is considerable empirical support (Andor, Gerlach, \& Rist, 2008;

Hofmann et al., 2005; Llera \& Newman, 2010, 2014; Lyonfields, Borkovec, \& Thayer, 1995; Peasley-Miklus \& Vrana, 2000; Stapinski, Abbott, \& Rapee, 2010; Vrana \& Lang, 1990). The theory and related supporting evidence suggest that people with GAD worry to sustain a negative emotional state as this prevents an unexpected shift from a positive or neutral emotional state to a negative emotional state. In other words, people with GAD may opt for a sure negative emotional state over being uncertain about whether they will suddenly experience a worsening in their emotional state. This is consistent with the current result that people with GAD preferred to take a bad outcome over not knowing how things would resolve. Taken together, these findings emphasize how difficult not knowing is for people with GAD to tolerate.

Uncertainty allergy in GAD. The results showed that most people with GAD said that they are "allergic" to uncertainty, in that they have a large response to even a small amount of 
uncertainty. This result might be considered similar to the other finding that people with GAD feel abnormal when uncertain, in that they say that they react much differently and more negatively in response to uncertainty than do others. The finding that people with GAD report feeling allergic to uncertainty also dovetails with the results that people with GAD describe being bothered by their own responses to uncertainty and report a negative attitude toward not knowing even when consequences are highly unlikely and in some cases also when consequences are minor. A description of the allergy analogy is currently included in Dugas and Robichaud's (2007) CBT-IU manual for GAD (p. 118) to help people with GAD understand what is meant by the term "intolerance of uncertainty." The finding that the allergy analogy "fits" for most people with GAD supports its use in clinical practice.

On the other hand, non-psychiatric control participants said that they were not allergic to uncertainty. Indeed, people in the NPC group tended to say that they were "okay" with uncertainty and some even mentioned that they believe some uncertainty in life to be healthy. The finding that non-psychiatric control participants are not allergic to uncertainty is in line with the other study findings that most people in the non-psychiatric control group described thoughts of acceptance of uncertainty as part of their typical attitude toward uncertainty, as well as thoughts that they can generally cope with uncertain situations that arise. This result is further consistent with the result that the majority of non-psychiatric controls do not consider uncertainty to be personal, and have an optimistic or realistic view on how uncertain situations will resolve.

\section{Limitations}

The present study employed a mixed methods approach that is not commonly used in research on anxiety disorders to extend what is currently known about IU in people with GAD. One potential limitation is that the examples provided to individuals during the qualitative 
interview to illustrate what was meant by questions may have influenced responses. The present study attempted to mitigate this potential influence on responses by using the same examples across participants and by checking in with participants about whether their responses were typical in other contexts. However, it is still possible that initial examples provided to participants anchored their understanding of questions and their responses. This could be addressed through replication of the present findings using a wider variety of different interview questions or by using alternative methods (e.g., vignettes) that would verify the stability of the present results.

The current sample of 40 participants may also be viewed as a limitation. However, the number of participants recruited for the present study exceeds guidelines on the number of interviews that are necessary to achieve saturation, which is the point at which no new themes emerge in response to qualitative research questions (Guest et al., 2006; Kuzel, 1992). In addition, it is worth noting that the present study was adequately powered to detect statistically significant quantitative differences in themes between groups.

One argument that has been proposed against research that includes qualitative components is that its findings may be less "trustworthy" or its designs less rigorous than those of more traditional quantitative research (Rolfe, 2006). Although it is true that some qualitative approaches do not use objective standards to compare differences between groups or themes, there were several steps taken to bolster the objectivity of the current study's results. First, differences in responses reported by groups were verified using quantitative statistics, so that an objective standard was used to assess whether differences between groups were present or absent. Second, each theme described herein was documented in detail in a codebook, and then each participant's responses were checked for the presence of the themes. A codebook was 
created to help ensure that the themes found in the interviews of the present study were replicable. Moreover, using this codebook approach, half of the interviews collected in the present study were randomly selected for coding by a second independent rater, and interrater reliability was found to be excellent. This suggests that the themes reported in the present study are objective and replicable.

The present study compared responses to questions about uncertainty in people with GAD relative to non-psychiatric control participants in order to provide a starting place for deepening the current understanding of IU in people with GAD. It may be considered a limitation that people with GAD were not compared to another group of people with high IU (e.g., people with SAD) in the present study. However, given the resources available for the present study, and the goal of exploring experiences of uncertainty in people with GAD relative to people without a psychological disorder in an in-depth manner, the inclusion of additional comparison groups would have compromised the detail that could be obtained in this study. Moreover, nuanced aspects of the experience of uncertainty in individuals without a psychological disorder are not yet well understood. It is important to understand what constitutes a "normal" attitude toward uncertainty in order for practitioners to appreciate what aspects of attitudes toward uncertainty are typical in people with GAD. Overall, the current study provided novel information about the phenomenology of IU that contributes to GAD theory and clinical practice. The present study supports the feasibility of using qualitative or mixed methods approaches to further explore the IU construct in future research.

\section{Future Directions}

The present study elucidated several novel insights from the perspectives of people with GAD and non-psychiatric control participants. These discoveries point to numerous future research directions, some of which are highlighted below. 
Being bothered by uncertainty itself. The current study pointed out that people with GAD seem to find uncertainty upsetting both when there are no threatening outcomes (i.e., positive/neutral uncertainty) and when there could be negative outcomes. Given other current findings, it might be hypothesized that uncertainty itself is bothersome because it still elicits strong emotions in people with GAD, and potentially activates beliefs about being incapable or abnormal. These hypotheses remain to be tested in future research. Another crucial research question is whether being bothered by uncertainty itself is true of all diagnostic groups who report high levels of IU or specific to GAD.

Parameters of situational exposure to uncertainty. The present study highlights that not all "types" of uncertainty are equally intolerable to people with GAD. What remains unknown is how this information should be applied to treatments that are designed to foster a greater tolerance for uncertainty by repeatedly approaching uncertain situations (e.g., uncertainty exposure in CBT-IU). Future research may wish to investigate whether it is beneficial to use a "stepped" method to approaching uncertainty, wherein people begin by approaching uncertain situations that are more tolerable to them, and then move to approaching more anxiety-provoking uncertain situations. It would also be useful to know if individuals with GAD benefit more from confronting uncertainty with potential negative outcomes, or if the same treatment outcomes can be achieved by approaching positive or neutral uncertain situations (i.e., uncertain situations that lack threatening outcomes such as receiving a gift). In other words, can approaching feelings of not knowing for the purpose of improving IU be done with less “pain” and equal "gain”?

Beliefs that may maintain uncertainty aversion. The present study showed that people with GAD thought that being uncertain meant that they were ineffective and abnormal, and that uncertainty is unfair because others cope better with being uncertain and they don't deserve to be 
uncertain. The role that such beliefs may play in producing negative emotional states and aversion to, or nonacceptance of, uncertain situations remains to be studied. One hypothesis is that strongly activated negative beliefs about uncertainty, such as “I don't deserve this” or "I can’t cope” may heighten arousal and prevent a person from handling an uncertain situation skillfully, further perpetuating IU beliefs. Future research testing how the beliefs uncovered in the present study may maintain worry and IU would extend the current theoretical understanding of GAD.

Optimizing measures of IU. Several attitudes about IU were discovered in the present study, and the degree to which current IU assessments tap such attitudes has not yet been studied systematically. For example, it is unclear whether current measures of IU adequately assess beliefs that uncertainty: 1) will resolve negatively, 2) is intolerable because of the feelings it produces, 3) indicates that one is incompetent and abnormal, 4) is unfair because others are not uncertain and it is undeserved, and 5) is bothersome in the absence of negative outcomes. Future research should strive to clarify what the IU construct is, and how best to measure it. The beliefs highlighted above point to potential aspects of the IU construct; however, further study is required to determine whether the inclusion of such specific beliefs in measures of IU, such as the IUS, would improve its assessment.

Links among theoretical models of GAD. Several findings in the current study highlighted potential connections between the IU Model of GAD (Dugas et al., 1998) and other theoretical models of GAD. For instance, people with GAD said that uncertainty was intolerable because of the emotions it provokes, suggesting a link between IU theory and the Emotion Dysregulation Model of GAD (Mennin et al., 2004). It is possible that uncertain situations may be the prompt for emotion dysregulation, and that IU may interact with emotion regulation 
difficulties to contribute to the maintenance of pathological worry. This hypothesis remains to be tested in future research. In addition, people with GAD said that they would rather have a definite bad outcome than be uncertain. This finding may be considered consistent with the Contrast Avoidance Model of GAD (Newman \& Llera, 2011) in that this theory suggests that people with GAD worry to maintain negative emotions in order to avoid uncertainty about suddenly experiencing a worsening in emotional state. Future research could test if attempts to be certain, even if that means accepting a negative outcome, are attempts to avoid sudden shifts from neutral or positive emotions to negative emotions. Given that there are several conceptual models of GAD in the literature, empirical study of how these theories relate to each other is an important way to further the current understanding and treatment of pathological worry.

\section{Summary and Conclusion}

There were several aspects about the experience of being uncertain that were unique to people with GAD, including assuming the worst case scenario when outcomes are unknown and experiencing uncertainty as personal, namely a reflection of one’s own incompetence, abnormality, or lack of preparation. People with GAD also provided more reasons why uncertainty is unfair than did non-psychiatric control participants. Furthermore, people with GAD said that uncertainty was a negative experience because of how they responded to it, and said they felt "allergic" to uncertainty. Unlike non-psychiatric control participants, most people with GAD said that they would worry to a similar extent regardless of how likely a feared outcome was to occur, and some people with GAD said that they would worry to the same degree regardless of how bad a feared outcome was. Furthermore, the majority of people with GAD said that not knowing, even when all potential outcomes are positive, would elicit negative feelings and expectations, and were bothered by uncertainty in and of itself (in the absence of negative outcomes). In sum, these findings indicate that people with GAD hold attitudes toward 
uncertainty that differ from people without a psychological disorder in a variety of ways and may serve to maintain pathological worry.

There were also numerous elements of being uncertain that people with GAD described that seemed to be typical, reported by an equivalent number of individuals without a psychological diagnosis. Individuals with GAD and non-psychiatric control participants described a desire to know the outcome when they were faced with uncertainty, although people with GAD described the need to know in qualitatively stronger terms. In addition, all individuals discussed possible negative outcomes as a reason why uncertainty is negative. Both groups said it can feel unfair to not know what will happen, and provided some of the same reasons why. Moreover, feeling unprepared when uncertain was described by people with GAD and people in the control group alike. Almost everyone said that uncertainty can be positive, and like nonpsychiatric control participants, those with GAD said that they feel positive emotions (albeit, alongside negative emotions) in response to uncertainty with several possible positive outcomes. All individuals said that they feel worse about not knowing when they are the only one not privy to information, and when uncertain situations are less in their control. Thus, there are several ways in which attitudes toward uncertainty in people with GAD are normative, which suggests that IU is not a fully pathological process.

Lastly, there were some attitudes described by non-psychiatric control participants that were not discussed by people with GAD. These included thoughts of acceptance when uncertain, believing uncertainty is not personal or even has positive personal meanings, and being optimistic when uncertain. In addition, healthy people's attitudes toward the unknown are based on how likely or severe possible negative outcomes are. Further, most people without a psychological disorder said that they would prefer to remain uncertain than to settle for a sure 
negative outcome and said that they were not "allergic" to uncertainty. In sum, there were attitudes toward uncertainty that were unique to the non-psychiatric control group, and suggest that although there is overlap in how people with and without GAD experience uncertainty, there are also areas of divergence.

Overall the results suggest that helping individuals with GAD to consider outcomes other than the worst case scenario and to "depersonalize” the meaning of uncertainty may assist in reducing negative beliefs about uncertainty, potentially improving pathological worry. Furthermore, gaining experience accepting uncertainty and skillfully navigating uncertain situations may help people with GAD to challenge negative beliefs about their own reactions to uncertainty and their coping abilities when uncertain. Future research may seek to more thoroughly understand how and why non-psychiatric control participants accept uncertainty and feel competent when uncertain, as this could inform how best to deliver treatments that seek to help people with GAD become more comfortable with uncertainty in order to worry less. 


\section{Study 1b: Behavioural Responses to Uncertainty in People with GAD and Non-Psychiatric Control Participants}

\section{Introduction}

Cognitive behavioural theories of anxiety disorders propose that the way people think and what people do (or do not do) play a role in maintaining anxiety (e.g., Beck et al., 1985; Clark \& Beck, 2010). One behaviour that is common across the anxiety disorders is avoiding the object or situation that is perceived to be threatening, which maintains anxiety as it prevents learning that a feared object or situation is not associated with catastrophic outcomes, and that a person can cope with the object or situation (see Clark \& Beck, 2010; Hofmann, 2008). Another way of acting that can perpetuate anxiety is using "safety behaviours," defined as behaviours that are performed in order to prevent or minimise a feared catastrophe (Salkovskis, 1991). For people with social anxiety disorder, an example of a safety behaviour may be speaking to others only after rehearsing what to say, and for people with spider phobia, a safety behaviour may be wearing hooded shirts in places where spiders are likely to be found. Safety behaviours can maintain anxiety by interfering with learning that feared consequences do not occur even when safety behaviours are not used, and that a person can cope effectively even without the use of compensatory actions (see Clark, 1999 for a review). In sum, avoidance and safety behaviours are important factors that contribute to the continuation of anxiety difficulties; however, behaviours that people with GAD engage in that may perpetuate excessive worry are not yet well understood.

There is substantial support for the role of IU in GAD (see Carleton et al., 2012; Dugas et al., 2010; Gentes \& Ruscio, 2011; Koerner \& Dugas, 2008; Yook et al., 2010), although very little is known about potential behavioural elements of IU. To date, the IU theory of GAD has emphasized the role of cognitive processes that may underlie and maintain worry, including 
negative beliefs about uncertainty, a negative view of problem solving, positive beliefs about worry, and cognitive avoidance (e.g., Dugas et al., 1998, 2007). It is of note that although cognitive avoidance may be considered a cognitive process, it may involve some behavioural elements, such as avoiding situations that provoke worrisome thinking (see Gosselin et al., 2002; Sexton \& Dugas, 2008). Overall, however, the potential role of behavioural responses to uncertainty in maintaining pathological worry has received limited attention. One way to further the IU theory of GAD is by investigating behaviours that individuals engage in when they are uncertain.

In addition to scarce data on behaviours in the context of the IU model of GAD, there is a general dearth of research on the behaviours of people with GAD relative to what is known about the cognitions of people with GAD. Exemplifying the lack of emphasis on behaviours, unlike other anxiety-based disorders such as social anxiety disorder or obsessive-compulsive disorder, GAD does not include behaviours as part of its diagnostic criteria at the present time (APA, 2013). Indeed, although behavioural criteria were proposed for inclusion as part of the GAD diagnosis in DSM-5³ (Andrews et al., 2010), these criteria were ultimately rejected due the limited research to date on specific behavioural correlates of GAD (see APA, 2013; Andrews et al., 2010; Regier, 2010; Starcevic \& Portman, 2013). Thus, there is a general need to better understand what the "safety behaviours" and avoidance behaviours are in people with GAD, and how these relate to established theories and disorder maintenance. The present study begins to

\footnotetext{
${ }^{3}$ The proposed behavioural criteria for GAD included: avoiding potentially negative events, over-preparing for potentially negative outcomes, procrastination due to worries, and reassurance-seeking due to worries (see Andrews et al., 2010).
} 
address this by examining the behaviours of people with pathological worry in the context of Dugas et al.’s (1998) IU theory.

A few studies provide a starting point for examining behaviours that may be relevant in GAD. A recent study by Beesdo-Baum and colleagues (2012) found that compared to nonpsychiatric control participants, people with GAD reported more frequent engagement in reassurance-seeking, cognitive avoidance (e.g., thought control), behavioural avoidance (e.g., avoiding worrisome situations), and acts that the authors labeled safety behaviours (e.g., overplanning, information seeking). Although Beesdo-Baum et al. assessed the frequency with which individuals with GAD engaged in these various behaviours in general, what remains unknown is the degree to which people with GAD employ these behaviours in response to uncertainty. Research has yet to demonstrate the connection between IU and behaviours that appear to be frequent in GAD.

Studies on procrastination and evidence requirements also shed light on behaviours that may be linked to excessive worry. For instance, Stöber and Joormann (2001) found that greater worry was associated with more procrastination in two undifferentiated samples ( $r s=.32$ to .41 ). Borkovec, Hazlett-Stevens, and Diaz (1999) have also observed through clinical practice that many people with GAD engage in procrastination. In addition, individuals who worry excessively have been found to require more evidence before making decisions compared to people who are low on the tendency to worry, which may signal a tendency toward excessive information seeking in people who worry more (Tallis, Eysenck, \& Mathews, 1991). Consistent with this, Ladouceur, Talbot, and Dugas (1997) found a significant association between participants' IU scores and the amount of information they required before responding to a moderately ambiguous task. Rosen and colleagues $(2009,2010)$ also found that higher IU was 
associated with greater information seeking. To summarize, research suggests that procrastination and information seeking are elevated in individuals with higher worry or IU.

Furthermore, IU has been associated with a few covert, mental, or unobservable strategies that may be used to control uncertainty, including worrying and cognitive avoidance strategies (e.g., avoiding mental images of fearful scenarios, replacing negative thoughts with positive or neutral thoughts; Borkovec \& Inz, 1990; Borkovec, Ray, Stober, 1998; East \& Watts, 1994). For the purposes of the present study, these strategies will be considered behaviours, or actions that individuals may take in response to uncertainty, recognizing that such strategies are indeed mental, and could also be conceptualized as cognitions. As has been described throughout this dissertation, there is a large body of research suggesting that people higher in IU tend to worry more (e.g., Dugas et al., 1998, 2007; Gentes \& Ruscio, 2011). Research has also shown a significant moderate association between IU and cognitive avoidance strategies (Dugas, et al., 2005; Dugas et al., 2007). Further, worry and cognitive avoidance are each theorized to be potential consequences of higher IU in individuals with GAD (Dugas et al., 1998, 2007). Thus, worry and cognitive avoidance may be strategies that individuals with GAD engage in to cope with uncertainty.

Clinical writings have also proposed a number of possible behavioural manifestations of IU. Dugas and Robichaud (2007) categorize such behaviours into "approach strategies” (e.g., reassurance seeking, excessive checking, and excessive information seeking) and "avoidance strategies” (e.g., avoiding situations with unknown outcomes, not fully committing to things, and procrastinating). In line with this, other theorists have similarly categorized responses to threat into approach and avoidance dimensions (Krohne, 1993; Miller, 1987; Sorrentino \& Short, 1986). Miller (1987) described that people may respond to anxiety-provoking situations by 
“monitoring” (i.e., seeking threat-related information) which would be an approach strategy or "blunting” (i.e., avoiding threat-related information), which would be an avoidance strategy. Some behaviours that Dugas and Robichaud (2007) propose in their theoretical writing have been empirically linked to GAD symptoms (e.g., procrastination; Stöber \& Joormann, 2001). However, it is currently unknown whether people with GAD engage in these strategies in response to being uncertain.

In sum, behaviours that have been linked to GAD (e.g., reassurance-seeking, excessive information seeking; Beesdo-Baum et al., 2012; Tallis et al., 1991) may play an important role in perpetuating GAD symptoms, but have not yet been investigated in the context of IU. Such information has the potential to extend Dugas et al.'s (1998) IU Model of GAD, as this theory currently does not emphasize overt (i.e., observable) behavioural factors that may contribute to or maintain pathological worry. Furthermore, a better understanding of behaviours that people with GAD engage in to cope with uncertainty may inform clinical interventions. As described in Study 1a of this dissertation, one of the treatment techniques used in CBT-IU for GAD involves identifying and changing behavioural responses to uncertainty (Dugas \& Robichaud, 2007; Robichaud, 2013); however, to date there is a limited empirical understanding of what these behaviours are. A scientific understanding of common behavioural manifestations of IU would provide direction for practitioners using CBT-IU for GAD, as it would guide what behaviours to ask clients about, facilitating the identification of behavioural treatment targets.

In addition, the most commonly used measure of IU in the GAD literature, the Intolerance of Uncertainty Scale (IUS; Buhr \& Dugas, 2002), assesses principally beliefs and appraisals of uncertainty. Although the IUS includes two items that assess behavioural responses to uncertainty, namely avoiding uncertainty and not going forward when uncertain (i.e., "doing 
nothing”), the IUS does not measure a range of potential behavioural responses to uncertainty. More recently, the Intolerance of Uncertainty Index was developed by Gosselin and colleagues (2008), and measures three potential behavioural responses to uncertainty; avoidance of uncertainty, reassurance seeking, and attempts to control uncertainty (Gosselin et al., 2008; English translation: Carleton, Gosselin, \& Asmundson, 2010). Thus, although some behavioural responses to uncertainty are included in current IU measures, the number of behaviours assessed is limited, and knowing more about behavioural responses to uncertainty may allow for the expansion of current measures or the development of new measures.

The present study also sought to provide information on the degree to which uncertaintyrelated behaviours in GAD are normative by including a group of non-psychiatric control participants (NPCs). It is often assumed that the way that people with GAD behave is maladaptive; however, the degree to which such behaviours are "normal" is not well understood. Knowing more about what behaviours in emotional disorders are pathological versus normative can help inform theory and treatment. For example, research on obsessive-compulsive disorder (OCD) has shown that most healthy people experience the same repugnant, intrusive thoughts that people with OCD grapple with (Clark \& Inozu, 2014; Rachman \& de Silva, 1978); however, the difference between individuals with OCD and nonclinical controls lies in a person's appraisal of those thoughts and the need to neutralize in response to the thoughts (e.g., Clark, 2004; Rachman, 1997; Salkovskis, 1985). As such, the goal of CBT for OCD is not to modify intrusive thoughts themselves, but to change one's interpretation of the intrusive thoughts (Abramowitz, 2009; Clark, 2004). Relatedly, the goal of CBT for GAD may not be to eliminate behavioural responses to uncertainty, but rather, to reduce their frequency. 


\section{Present Study}

The present study compared behavioural responses to uncertainty in individuals with GAD to NPCs in order to understand the behaviours of people with GAD in the context of what is normative. As with Study 1a of this dissertation, which explored attitudes toward uncertainty, the present study used a mixed methods research approach which included qualitative and quantitative elements. Individuals with GAD and NPCs were asked open-ended questions about their behavioural responses to uncertainty, which produced qualitative data, and these data were further compared using quantitative methods. . The current study also included quantitative components when warranted by the study aim.

The present study's objectives were to investigate: 1) what behaviours individuals engage in when uncertain, and how often people engage in these behaviours; 2) the function of behavioural responses to uncertainty; 3) factors that lead to the discontinuation of behavioural

responses to uncertainty; and 4) whether behavioural responses to uncertainty cause problems for people, and if so, what kind of problems. To the best of my knowledge, these questions are completely novel, and have not yet been investigated in individuals with GAD in the context of understanding IU.

\section{Objective 1. Form and Frequency of Behavioural Responses to Uncertainty}

Understanding what behaviours people with GAD engage in when they are uncertain, and how often, was of interest as these behaviours may represent components of IU that have not yet been identified. Knowing more about behaviours that people with GAD engage in when uncertain is also expected to provide guidance to practitioners using CBT-IU for GAD, which involves identifying and addressing behavioural manifestations of IU (see Dugas \& Robichaud, 2007; Robichaud, 2013). 
The nonclinical literature on ambiguity aversion, a construct that is conceptually related to IU, suggests that most people try to avoid uncertain situations (see Al-Najjar \& Weinstein, 2009 for a review). However, when avoidance is not possible, people typically seek to reduce uncertainty through information-gathering (Berger \& Calabrese, 1975; Brashers et al., 2000; Kramer, 1999; Lipshitz \& Strauss, 1997). Similar to the nonclinical literature, clinical writings suggest that individuals with GAD react to uncertainty with two types of strategies: "avoidance strategies” (i.e., behaviours that reflect not wanting to know, such as avoidance or procrastination) and "approach strategies” (i.e., behaviours that reflect a need to know, such as excessive reassurance-seeking or information gathering; Dugas \& Robichaud, 2007). It is of note that approach and avoidance strategies are not diametrically opposed, and the same individual may engage in both behaviours when faced with uncertainty. However, because others have grouped behaviours in this way (e.g., Dugas \& Robichaud, 2007; Dugas \& Koerner, 2005; Muris, Van Zuuren, De Jong, De Beurs \& Hanewald, 1994; Van Zuuren \& Wolfs, 1991), I refer to these ways of classifying behaviour in the present study as well.

It was expected that the GAD and NPC groups would report "approach" and "avoidance” behaviours. However, given that individuals with GAD are known to have more difficulty tolerating uncertainty than do people low in worry and anxiety (see Dugas et al., 1998, 2007), it was hypothesized that individuals with GAD would describe a greater range of behavioural responses to uncertainty than would non-psychiatric control participants. In addition, based on research suggesting that individuals with GAD engage in behaviours such as reassurance-seeking more often than individuals without a psychological disorder in general (Beesdo-Baum et al., 2012), individuals with GAD were similarly expected to report employing these behaviours more frequently than were non-psychiatric controls in response to uncertainty. 


\section{Objective 2. Function of Behavioural Responses to Uncertainty}

Examining the perceived function of behavioural responses to uncertainty was of interest as it has the potential to inform the IU model of GAD by providing insights about how these responses are reinforced (i.e., what people "get out of" doing these behaviours). Knowing more about the function of behavioural responses to uncertainty may also help clinicians working with people with GAD to better understand their clients, and to validate the needs addressed by the behaviours that people with GAD employ when uncertain.

It has been proposed that individuals with GAD may approach uncertainty in an effort to increase certainty, and avoid uncertainty in order to reduce negative emotions (Dugas \& Koerner, 2005). Dugas and Koerner theorized that individuals with GAD may have a need to both approach uncertainty in order to resolve it, and at the same time, to avoid uncertainty when feelings elicited by the unknown become too overwhelming. They further suggested that people with GAD may find themselves caught in an "approach-avoidance conflict,” paralyzed by competing needs "to know” and "not to know."

Accordingly, themes reflecting a need to “increase certainty” and "reduce negative emotions” were expected to emerge in participant responses as functions of behavioural responses to uncertainty. Since individuals with GAD are higher in IU than are people low in worry and anxiety (Dugas et al., 1998), more people in the GAD group compared to NPC group were expected to offer responses consistent with these themes. As this is the first known study of the function of behavioural responses to uncertainty in individuals with GAD, no other $a$ priori hypotheses were proposed, although novel themes were expected to emerge. 


\section{Objective 3. Factors that Lead to the Discontinuation of Behavioural Responses to Uncertainty}

The present study investigated what leads individuals with GAD and NPCs to stop engaging in the main behaviours that they engage in when uncertain, as well as factors that lead both groups to stop engaging in worry, given that worry is the cardinal feature of GAD (APA, 2013) and is proposed to be a response to uncertain situations. Information about cessation factors may be clinically useful if people with GAD describe different requirements to stop behavioural responses to uncertainty than NPCs. It may be helpful to identify and assist people with GAD with changing what they require before stopping behavioural responses to uncertainty; for instance, if a person feels that he or she needs to be certain before stopping behaviours like overpreparing, then this might be challenged in treatment. Understanding what leads people with GAD and NPCs to discontinue behavioural responses to uncertainty may also provide theoretical insights about GAD by highlighting beliefs or "rules” that may lead behaviours to persist.

People higher in worry have been found to require excessive information before making a decision compared to people lower in worry (e.g., Ladouceur et al., 1997). Extrapolating from this finding, it was hypothesized that compared to NPCs, more individuals with GAD would describe engaging in behavioural responses to uncertainty, including worry, until a feeling of certainty is achieved. Given the limited research to date on factors that stop behavioural responses to uncertainty, novel themes were also expected to emerge.

\section{Objective 4. Impairment Caused By Behavioural Responses to Uncertainty}

Although it is assumed in related research (e.g., Ladouceur 1997) and clinical writings (e.g., Dugas \& Robichaud, 2007; Robichaud, 2013) that the behaviours that people with GAD engage in when uncertain are problematic, it is currently unclear whether individuals with GAD 
perceive this to be the case. If IU-related behaviours cause problems for people with GAD, then this further supports investigating the role of such behaviours in maintaining GAD. In addition, knowing whether IU-related behaviours are problematic from the perspective of people with GAD may provide clinicians with ideas about questions to ask clients (e.g., how has difficulty tolerating uncertainty affected your relationships?) to elicit potential reasons to make changes to behavioural responses to uncertainty over the course of CBT-IU.

Given the difficulties associated with GAD (Hofmann et al., 2008), and the behaviours that individuals with GAD are theorized to engage in when uncertain (e.g., procrastination; Dugas \& Robichaud, 2007), it was expected that more people with GAD than NPCs would report that IU-related behaviours are problematic for them. Research has shown that excessive reassurance seeking can strain relationships (e.g., Joiner \& Metalsky, 2001; Potthoff, Holahan, \& Joiner, 1995), and that individuals who procrastinate more tend to perform worse on their work or school goals, and get sick more often than do those who procrastinate less (e.g., Steel, 2007; Tice \& Baumeister, 1997). Thus, it was hypothesized that individuals with GAD would report that their behavioural responses to uncertainty would cause difficulties with relationships, work or school, and physical health. Given the limited research on this topic, it was also expected that participants may raise novel themes about ways in which behavioural responses to uncertainty are problematic for them. It was assumed that people with GAD would be able to report on problems related to behavioural responses to uncertainty as to the best of my knowledge low insight (or delusionality) as has not been identified as a feature of GAD, as it has been with some other difficulties such as obsessive-compulsive disorder and body dysmorphic disorder (e.g., Eisen, Phillips, Coles, \& Rasmussen, 2004). 


\section{Method}

Study $1 \mathrm{~b}$ of this dissertation is the second part of a single, in-depth mixed methods investigation the addressed two sets of research questions - one about attitudes toward uncertainty (Study 1a) and the other about behavioural responses to uncertainty (the present study). Please see the Method section of Study 1a of this dissertation for information about: participants (pp. 28-33), measures (pp. 33-38), qualitative interview guide development (pp. 3839), coding approach and codebook development (pp. 39-41), and the procedure (p. 41, 43). Aspects of the Methods section that were specific to Study 1b are described below.

\section{Measures}

\section{Qualitative Interview.}

A semistructured interview guide was developed for the present study, which included sets of questions designed to address the current study’s research objectives. See qualitative interview guide development in Study 1a for more details and Appendix B for full details of the interview guide questions. A summary of key questions is presented below.

1. Form (Open-Ended Question). Participants were asked to think of a specific uncertain situation that they found themselves in recently, where they acted in a way that was typical of them. Here an uncertain situation was defined as "a situation where you felt unsure of what to do, or unsure about how something would turn out.” Then participants were asked what they did or did not do in response to the uncertain situation in an open-ended manner.

2. Frequency (Closed-Ended List). A list of possible behavioural responses to uncertainty was developed based on various sources, including: 1) behaviours described in Dugas and Robichaud's (2007) CBT for GAD manual, 2) theoretical writings on GAD (e.g., Dugas \& Koerner, 2005), 3) clinical research on the behaviours of people with GAD or high worry (Beesdo-Baum et al., 2012; Gosselin et al., 2008; Pawluk \& Koerner, 2013; Sexton \& Dugas, 
2008; Stöber \& Joormann, 2001; Tallis et al., 1991), and 4) nonclinical research on uncertaintyrelated behaviours (Berger \& Calabrese, 1975; Brashers et al., 2000; Inglis 2000; Kramer, 1999; Krohne, 1993; Lipshitz \& Strauss, 1997). Participants were asked about whether they had engaged in each behaviour on the list when they were uncertain over the past month (yes or no). If participants responded yes, they were asked to estimate how many times they had engaged in the behaviour over the past month.

3. Function. The interviewer oriented participants by asking them to consider two or three behaviours that they had reported using most often in the Frequency section of the interview (see above). For their most frequent behaviours, participants were asked "what do you get out of doing ___ (the behaviours)"?

4. Discontinuation Factors. The interviewer oriented participants again by reminding them of the behaviours they had endorsed using most often in the Frequency section of the interview. For their most frequent behaviours, participants were asked "what allows you to stop doing ___ (the behaviours)"? Participants were also asked what allows them to stop worrying, given that worry is the core feature of GAD (APA, 2013) and is considered a coping behaviour that is triggered by uncertainty (Ladouceur et al., 2000; Rosen \& Knäuper, 2009).

5. Impairment. Participants were asked whether any of the behavioural responses to uncertainty that they had endorsed during the interview caused problems for them. If yes, participants were asked to describe the problems caused by the behaviour(s). 


\section{Qualitative Approach}

Qualitative interview guide development. See Study 1a for full information about the development of the interview guide (pp. 38-39). An iterative approach was taken to the development of the semistructured interview guide, wherein updates were made to the interview after data collection commenced based on the responses offered by participants. Using an iterative approach to interview guide development is consistent with best practices in qualitative research (Flick, 2009). After data collection commenced, this writer in collaboration with the research team decided to add several questions based on initial participant response patterns. A specific prompt was added to assess how often participants problem solve when uncertain, as initial participants spontaneously reported problem solving when asked if there were any other behaviours they engage in when uncertain. Given initial differences between people with and without GAD in terms of their responses to uncertainty with positive outcomes, a question was also added to assess how often individuals “embrace” or make the best of uncertainty. In addition, questions about impairment associated with behavioural responses to uncertainty were added after data collection had commenced, based on initial findings of how frequently individuals with GAD engaged in behavioural responses to uncertainty.

\section{Reliability.}

All interviews were coded by the same primary rater (KF), and 50\% of the sample (20 interviews) were randomly selected and coded independently by a second rater to determine interrater reliability. The second rater was blind to group membership. The average interrater reliability of all research questions in the present study was 99.88\% agreement, and had a Cohen’s Kappa (к) of .77. Cohen’s Kappa’s above .75 are considered excellent (Fleiss et al., 1981), as $\kappa$ is a conservative test that takes into account $\%$ agreement and $\%$ disagreement. 
The $\%$ agreement and $\kappa s$ for each research question were as follows: form $(\%$ agreement $=$ 99.91; $\kappa=.83)$, function $(\%$ agreement $=99.84 ; \kappa=.70)$, general discontinuation factors $(\%$ agreement $=99.92 ; \kappa=.73)$, worry discontinuation factors $(\%$ agreement $=99.86 ; \kappa=.79)$, and impairment $(\%$ agreement $=99.89 ; \kappa=.82)$. Given the high interrater reliability, and the large number of themes in the present study, a decision was made to report the coding of the primary rater, as opposed to resolving each discrepancy through discussion. 


\section{Results}

\section{Objective 1. Form and Frequency of Behavioural Responses to Uncertainty}

Form (open-ended question). Participants were asked in an open-ended manner to describe their actions in response to an uncertain situation where they acted in a way that was typical of them. Table 8 shows the themes of the behavioural responses to uncertainty that the GAD and NPC groups described. It was hypothesized that behaviours would fall into approach and avoidance categories, and that individuals with GAD would describe using a greater range of behavioural responses when uncertain than would NPCs. Supporting this, most themes fell into approach and avoidance categories, and individuals with GAD said that they engaged in a significantly greater range of different behaviours as part of their typical response to uncertainty $(M=3.40, S D=1.32)$ compared to NPCs $(M=1.65 ; S D=0.88), t(38)=4.96, p<.01$. In other words, participants with GAD provided a greater number of behaviours than NPCs when asked about their typical response to uncertainty.

Approach Strategies. Based on clinical writings (e.g., Dugas \& Robichaud, 2007), the following behaviours were categorized as approach strategies: overprepare, check things over repeatedly, seek reassurance, and reassure oneself. Many people with GAD (50\%) described overpreparing as part of their typical response to uncertainty, whereas only 5\% of NPCs described behaviours that were consistent with this theme. Examples were: "I prepared and prepared and overprepared with getting everything in order"; "I put too much thought, too much work into things, and miss out on other things.” Similarly, $40 \%$ of the GAD group expressed checking things over repeatedly to be more certain, whereas $0 \%$ of NPCs described this. For example: "I know they only update the website once a day, but I would constantly check...I mean weirdly.” 
Table 8

Typical Behavioural Responses to an Uncertain Situation

\begin{tabular}{|c|c|c|c|}
\hline Themes & $\begin{array}{l}\text { GAD group } \\
(n=20)\end{array}$ & $\begin{array}{l}\text { NPC group } \\
(n=20)\end{array}$ & $\chi^{2}$ \\
\hline \multicolumn{4}{|l|}{ Approach Strategies } \\
\hline Overprepare & $50 \%$ & $5 \%$ & $10.16^{* *}$ \\
\hline Check things over repeatedly & $40 \%$ & $0 \%$ & $10.00 * *$ \\
\hline Seek reassurance from others & $35 \%$ & $15 \%$ & 2.13 \\
\hline Reassure self & $10 \%$ & $20 \%$ & 0.78 \\
\hline \multicolumn{4}{|l|}{ Avoidance Strategies } \\
\hline Avoid situations with an uncertain outcome & $40 \%$ & $10 \%$ & $4.80 *$ \\
\hline Distract self & $40 \%$ & $10 \%$ & $4.80 *$ \\
\hline Do nothing (i.e., become paralyzed by uncertainty) & $30 \%$ & $0 \%$ & $7.06 * *$ \\
\hline \multicolumn{4}{|l|}{ Other Strategies } \\
\hline Worry & $85 \%$ & $25 \%$ & $14.55^{* *}$ \\
\hline Problem solve & $25 \%$ & $90 \%$ & $17.29 * *$ \\
\hline
\end{tabular}

Note. Participants were asked to generate an example of an uncertain situation they had been in over the past week where they acted in a way that was typical of them. Participants described what they did or did not do as part of their response to uncertainty. The responses spontaneously described by participants were coded into the themes displayed above. Participant responses were often consistent with more than one theme, and thus percentage totals do not add up to $100 \%$. Individuals with GAD described significantly more behaviours as part of their typical response to uncertainty $(M=3.40, S D=1.32)$ compared to NPCs $(M=1.65 ; S D=0.88), t(38)$

$=4.96, p<.01$.

$*_{p}<.05, * * p<.01$. 
A comparable number of people in the GAD and NPC group reported seeking reassurance from others and reassuring themselves as part of their typical response to uncertainty. Examples of responses consistent with the seek reassurance from others theme included: "Usually it’s my mom that I talk to, because I do need some direction, or someone else to provide me with a certain amount of certainty to deal with the situation" (GAD) and "I called two of my girlfriends and told them about it. They helped me work through it and gave me the reassurance that I will get the second interview, that they really liked me, and that I was qualified for the position.” (NPC). Sample responses from the reassure myself theme included: "I just kept telling myself that I was prepared and a good candidate for a position” (GAD) and "I talk to myself and say okay you can do this or you are okay” (NPC).

Avoidance Strategies. The following behaviours were coded as avoidance strategies: avoiding situations with uncertain outcomes, distracting oneself from the uncertain situation, and doing nothing when uncertain. When asked about their typical responses to uncertainty, significantly more individuals in the GAD compared to NPC group discussed avoiding uncertain situations. Examples include: "kind of just like running away from the problem instead of trying to solve it” (GAD) and “I just walk away and don’t really deal with it” (NPC). In addition, significantly more individuals with GAD described distracting themselves when uncertain compared to the NPC group. For instance: “Distract myself with like going out or calling friends” (GAD) and “Oh I watched a DVD just to sort of get away for a minute and relax” (NPC). One third of individuals with GAD described doing nothing or being paralyzed in response to uncertainty, whereas none of the non-psychiatric control participants described this. Examples of the do nothing theme included: “it like brings me to a halt like I can’t do anything” and "it’s almost as if my life comes to a standstill." 
Other Strategies. Worrying and problem solving were described as typical responses to uncertainty, and were classified as "other" strategies as they are not clearly approach or avoidance behaviours, as defined by Dugas and Robichaud (2007). Most people with GAD (90\%) described worrying as part of their typical response to uncertainty, whereas this was true of only $20 \%$ of NPCs. For example: "I worry, and I keep going through things in my head" (GAD) and "worrying about the outcomes" (NPC). On the other hand, most NPCs (90\%) described problem solving as part of their usual response to uncertainty, whereas this was true of significantly fewer people with GAD (25\%). People said: "I think about all the possible outcomes and the possible steps that can be taken, and take the one that best fits the situation" (GAD) and "I tend to weigh different options and see what is the best way to handle the uncertainty" (NPC).

Frequency (closed-ended list). Next, participants were asked whether they had engaged in any of a list of behaviours when they were uncertain over the past month, and if so, how frequently. It was hypothesized that individuals with GAD would engage in behaviours in response to uncertainty significantly more often than NPCs. Consistent with this prediction, Table 9 shows that, with some exceptions, individuals with GAD employed behavioural responses when uncertain significantly more often than did NPCs. However, there were no significant differences in the frequency with which people with GAD and NPCs engaged in the following: seeking a lot of information, acting quickly without thinking things through, replacing negative thoughts with positive or neutral thoughts, and not fully committing to projects or activities with an uncertain outcome. 
Table 9

Frequency of Behaviours Participants Engaged in When Uncertain over the Past Month

Over the past month when you were uncertain, how often did you:

Approach Strategies

Repeatedly check things over (i.e., check things more than once)

Reassure yourself that everything would be okay or that you did everything correctly

Doubt and reconsider decisions that you had already made (i.e., repeatedly think about decisions already made)

Overprepare (i.e., spend more time than you thought was actually

necessary planning or preparing for something)

Overestimate the chance that bad things would happen

Seek reassurance from others that why you did was okay (past)

Seek reassurance from others about how things would turn out (future)

Maintain a specific routine

Overprotect others (i.e., call your adult child often to check to see that they were "okay")

Try to control everything in general (i.e., try to control what others do)

Not delegate tasks to others and do everything yourself

Seek out a lot of information (i.e., more information than most people would seek out)

Rehearse what you would say or do in a situation

Do things others that they could do themselves (e.g., make appointments for a partner)

Make work-related or personal commitments that you do not really want to make (e.g., take on more than you would like to)

Make decisions very quickly, without carefully weighing the options

\begin{tabular}{|c|c|c|}
\hline $\begin{array}{c}\text { Frequency of } \\
\text { behaviour in } \\
\text { GAD group } \\
M(S D)\end{array}$ & $\begin{array}{c}\text { Frequency of } \\
\text { behaviour in } \\
\text { NPC group } \\
M(S D)\end{array}$ & $\begin{array}{c}\text { Mann- } \\
\text { Whitney U }\end{array}$ \\
\hline $48.63(72.06)$ & $5.50(9.23)$ & $61 * *$ \\
\hline 32.73 (36.93) & $10.03(17.00)$ & $97 * *$ \\
\hline 29.38 (92.31) & $1.18(1.48)$ & $34.5^{* *}$ \\
\hline $28.20(65.05)$ & $2.63(6.74)$ & $71.5^{* *}$ \\
\hline $25.03(52.44)$ & $3.70(7.51)$ & $81 * *$ \\
\hline 23.70 (32.94) & $4.18(6.45)$ & $80 * *$ \\
\hline 16.45 (19.59) & $1.68(4.47)$ & $48 * *$ \\
\hline $15.80(17.36)$ & $3.73(9.12)$ & $118^{*}$ \\
\hline $13.18(27.76)$ & $0.70(1.46)$ & $94.5^{* *}$ \\
\hline 13.28 (12.37) & $2.10(4.30)$ & $84.5^{* *}$ \\
\hline $13.60(13.44)$ & 3.75 (7.97) & $106^{* *}$ \\
\hline 10.03 (11.18) & 6.70 (14.39) & 136.5 \\
\hline 8.78 (10.32) & $2.45(6.61)$ & $77.5^{* *}$ \\
\hline $5.53(9.12)$ & $0.80(1.82)$ & $111.5^{* *}$ \\
\hline $5.30(7.50)$ & $0.63(1.22)$ & $103.5^{* *}$ \\
\hline 3.90 (13.28) & $0.45(1.10)$ & 146.5 \\
\hline
\end{tabular}




\begin{tabular}{|c|c|c|c|}
\hline \multicolumn{4}{|l|}{ Avoidance Strategies } \\
\hline Try to push away thoughts of an uncertain situation & $17.65(20.90)$ & $0.78(1.26)$ & $55.50 * *$ \\
\hline $\begin{array}{l}\text { Thought replacement (i.e., try to replace negative thoughts with } \\
\text { positive/neutral thoughts) }\end{array}$ & $15.30(33.36)$ & $6.85(19.70)$ & 150.5 \\
\hline Procrastinate & $13.75(12.12)$ & $2.33(6.66)$ & $77 * *$ \\
\hline Distract yourself & $10.55(10.34)$ & $3.73(9.89)$ & $97 * *$ \\
\hline $\begin{array}{l}\text { Avoid activities with uncertain outcomes (e.g., avoid taking on a new role } \\
\text { at work, avoid trying something new) }\end{array}$ & $8.33(10.43)$ & $1.80(6.68)$ & $71^{* *}$ \\
\hline Take no action / do nothing & $6.83(9.41)$ & $0.30(0.68)$ & $100.5 * *$ \\
\hline Try not to imagine uncertain situations clearly & $7.58(26.81)$ & - & $150 *$ \\
\hline Not fully commit to projects or relationships with uncertain outcomes & $3.73(6.86)$ & $0.68(1.16)$ & 137.5 \\
\hline Take medication or use substances & $1.25(3.19)$ & - & $160 *$ \\
\hline \multicolumn{4}{|l|}{ Other Strategies } \\
\hline Worry & $48.90(95.81)$ & $4.00(6.63)$ & $18 * *$ \\
\hline Problem solve $^{\mathrm{a}}$ & $15.79(19.76)$ & $7.11(7.67)$ & 84 \\
\hline Embrace uncertainty (i.e., enjoy or make the best of uncertain situations) ${ }^{b}$ & $0.86(1.66)$ & $8.47(10.33)$ & $49.5^{* *}$ \\
\hline Average Frequency of All Strategies Assessed & $14.90(9.37)$ & $2.86(3.55)$ & $17.00 * *$ \\
\hline
\end{tabular}

Note. The behaviours listed in Table 9 were provided to participants, who were asked to rate the frequency with which they used each behaviour when they were uncertain over the past month. In the GAD and NPC groups, $100 \%$ of participants endorsed engaging in at least one behaviour from each of the "approach", "avoidance”, and “other” strategy category. The Mann-Whitney U test was used to compare differences in the frequency of behaviours between groups, as the data for all behaviours were non-normally distributed (right-skewed or bimodal distributions). The Mann-Whitney $U$ test has the same function as a traditional $t$-test, but does not assume a normal distribution. Note that covert/mental strategies were considered behaviours in the present study, although it is recognized that such strategies may be conceptualized as cognitions elsewhere.

${ }^{\mathrm{a}} n=14$ in the GAD group and 18 in NPC group. ${ }^{\mathrm{b}} n=14$ in the GAD group and 19 in NPC group. Unequal $n$ s were due to behaviours added after data collection had commenced.

${ }^{*} p<.05, * * p<.01$. 
Approach Strategies. The approach strategies that people with GAD engaged in most often when uncertain were: checking excessively, reassuring themselves, doubting and reconsidering decisions they had already made (i.e., repeatedly thinking about decisions made), overpreparing, and overestimating the chance that bad things would happen. Each of these behaviours was executed by individuals with GAD 28 to 48 times in the last month. The approach strategies that NPCs engaged in most often were: reassuring themselves, seeking information excessively, checking excessively, not delegating tasks to others, and maintaining a specific routine. Each of these behaviours was carried out by the NPC group three to 10 times per month. Compared to individuals in the non-psychiatric control group, individuals with GAD carried out almost all approach behaviours significantly more often over the past month, with the exception of seeking information excessively and making decisions quickly without carefully weighing the options, which were carried out with comparable frequency by people with GAD and NPCs. More information about the number of times that people in each group reported using each behaviour when uncertain can be found in Table 9 .

Avoidance Strategies. The avoidance behaviours that people with GAD engaged in most frequently were: pushing away thoughts about uncertainty, thought replacement, procrastinating, not paying attention to uncertainty, and distracting oneself from uncertainty. People with GAD reported engaging in each of these behaviours 10 to 17 times per month. For non-psychiatric control participants, the avoidance behaviours used most frequently were: thought replacement, distracting oneself from uncertainty, procrastinating, pushing away thoughts about uncertainty, and not fully committing to projects/relationships with uncertain outcomes. Non-psychiatric control participants reported engaging in each of these behaviours one to seven times per month. Compared to individuals in the NPC group, individuals in the GAD group carried out almost all 
avoidance behaviours significantly more often over the past month, with the exception of thought replacement and not fully committing to projects or relationships with uncertain outcomes, which were carried out with comparable frequency by people with GAD and NPCs.

Other Strategies. The interview also assessed the frequency of worrying, problem solving, and "embracing uncertainty" (i.e., enjoying uncertain situations or making the best of uncertain situations), which were classified as “other” strategies, as they were not clearly approach or avoidance behaviours. When individuals with GAD were uncertain, they engaged in worry significantly more frequently than individuals in the NPC group. People in the GAD and NPC groups reported engaging in problem solving to a comparable extent when uncertain over the past month. Individuals in the NPC group reported embracing uncertainty significantly more often than did individuals in the GAD group. Of all the behaviours that individuals were asked about, worry was the strategy that people with GAD engaged in with the highest frequency. On the other hand, reassuring oneself was the behaviour that non-psychiatric control participants engaged in most frequently, followed closely by problem solving, and embracing uncertainty. 


\section{Objective 2. Function of Behavioural Responses to Uncertainty}

The second objective of the present study was to assess the function of behaviours that individuals use when uncertain. It was hypothesized that more people with GAD would describe engaging in behavioural responses to uncertainty in order to reduce negative emotions and increase certainty. In line with this prediction, the functions of behavioural responses to uncertainty reported by participants were: to reduce distress, to increase certainty, to feel prepared for the worst, to optimize outcomes (i.e., use behaviours to achieve the best possible outcome), and to elicit support from others. The proportions of individuals who provided responses consistent with each theme by group are presented in Table 10.

A comparable number of people in the GAD and NPC groups described responses consistent with the reduce distress theme, although two qualitative differences were observed. First, 30\% of people with GAD described the reduction in distress produced by behavioural responses to uncertainty as partial or temporary. For example, “it makes you feel better, but not really, because what does she know? She's my friend not the decision maker" and "it lessens my anxiety, but I feel it's always temporary.” On the other hand, the notion of a partial or temporary reduction in distress was not apparent in the NPC group’s responses (0\%). For example, "Yes, absolutely [it makes me feel less anxious]” and “oh definitely, yes [it makes me feel better]”. Second, the GAD group (75\%) tended to frame the function of behavioural responses to uncertainty in terms of reducing negative emotion, for example, "it brings down the discomfort"; “it reduces my stress about it”; "I would feel less painful inside or less upset.” On the other hand, the NPC group (74\%) tended to frame the function of behavioural responses to uncertainty in terms of increasing positive emotion or wellbeing, for instance: "It makes me feel better”; “I feel more relaxed”; "It makes me feel more confident.” 
Table 10

Functions of Behavioural Responses to Uncertainty ${ }^{1}$

\begin{tabular}{lccc}
\hline Themes & $\begin{array}{c}\text { GAD } \\
(n=20)\end{array}$ & $\begin{array}{c}\text { NPC } \\
(n=20)\end{array}$ & $\chi^{2}$ \\
\hline To reduce distress & $100 \%$ & $95 \%$ & 0.00 \\
To increase certainty & $80 \%$ & $80 \%$ & $5.63 *$ \\
To feel prepared for the worst & $35 \%$ & $5 \%$ & 0.13 \\
To optimize outcomes a ${ }^{2}$ & $30 \%$ & $25 \%$ & 2.50 \\
To elicit support from others & $30 \%$ & $10 \%$ & \\
\end{tabular}

Note. Participants were asked to describe the function of the main behaviours they use in response to uncertainty. Participants often offered more than one response, thus percentage totals do not add up to 100. As participants were not asked about each theme specifically, it is possible that a given theme applied to a person, although the participant did not spontaneously report it.

${ }^{1} 100 \%$ of individuals in the GAD and NPC groups endorsed engaging in at least one behavioural response to uncertainty, and thus were able to discuss the function of such behaviours.

${ }^{a}$ Optimize outcomes refers to using behaviours in an attempt to achieve the best possible outcome (e.g., selecting the best possible item of clothing, attaining the best possible grade on a test).

$* p<.05$. 
For the increase certainty theme, there were no significant quantitative or qualitative differences between the GAD and NPC groups. Sample responses included: "the more information I get...the more certain I'll be" (GAD) and "going through these motions make me feel more certain” (NPC).

There was a significant difference between groups in the feel prepared for the worst theme, in that significantly more people with GAD described responses consistent with this theme than NPCs. Examples from with the GAD group were: “So that I won't be shocked. I’ll prepare emotionally... if that [negative outcome] happens, I know what I'm going to do, I don't have to panic" and "I'd rather prepare for something negative and overprepare for it as opposed to think it's going to turn out well and then have it turn out bad.” The one person from the NPC group who described a response that fit with this theme said: "it makes me feel prepared, and not like I will be caught off guard.”

There was no significant difference in the number of individuals from each group describing behaviours motivated by the need to attain the best possible outcome in a situation (i.e., optimize outcomes). Although there were no significant quantitative differences between groups, $50 \%$ of responses from the GAD group showed a strong degree of conviction about the necessity and positive function of engaging in uncertainty-related behaviours in order to optimize outcomes, whereas this was not true of the non-psychiatric control responses. Some examples from the GAD group exemplifying this point were: "I need to make sure I have all the information so I can do the best job possible" and "worrying is what makes me study for the exam.” The non-psychiatric control group’s responses did not illustrate the necessity of completing uncertainty-related behaviours in order to optimize outcomes, yet still highlighted reasons to use such behaviours, for example: "when I seek information it helps me make the 
decision easier and faster” and “I already know the situation isn’t going to be bad, but maybe I can make it better”.

Lastly, there were no quantitative or qualitative differences between groups in terms of the elicit support from others theme. Examples of responses consistent with this theme were: "I like to make sure other people agree with me, just for like support, to support my own thoughts” (GAD) and "that I might have another source of support through this, it makes me feel not alone and more confident” (NPC).

\section{Objective 3. Factors that Lead to the Discontinuation of Behavioural Responses to Uncertainty}

Discontinuation factors (general). The third goal of the present study was to understand factors that lead to stopping behavioural responses to uncertainty (see Table 11). It was expected that more people with GAD compared to NPCs would report wanting to feel certain before they stopped behaviours in response to uncertainty, including worry. Consistent with this hypothesis, for the cessation of behaviours in general, significantly more individuals with GAD compared to NPCs said they continue to engage in behavioural responses to uncertainty until the uncertain situation resolves in a definite outcome. Examples of how people with GAD described this included: "I think until I know it’s going to be ok... which usually doesn’t get there before something is resolved”; “I think I’m always anxious about being prepared until it's over with"; and "if the outcome is positive then you stop.” Non-psychiatric control participants offered responses with similar content, e.g., "it’s until the uncertainty is resolved.” 
Table 11

Factors That Lead To Discontinuing Behavioural Responses to Uncertainty

\begin{tabular}{|c|c|c|c|}
\hline Themes & $\begin{array}{c}\text { GAD } \\
(n=20)\end{array}$ & $\begin{array}{c}\text { NPC } \\
(n=20)\end{array}$ & $\chi^{2}$ \\
\hline \multicolumn{4}{|l|}{$\begin{array}{l}\text { When do you stop engaging in your main behavioural responses to } \\
\text { uncertainty? }\end{array}$} \\
\hline After uncertainty resolves & $70 \%$ & $15 \%$ & $12.38 * *$ \\
\hline Until it is no longer possible & $40 \%$ & $0 \%$ & $10.00 * *$ \\
\hline After I do it once or twice & $0 \%$ & $45 \%$ & $11.61^{* *}$ \\
\hline Until I have a subjective feeling to stop & $45 \%$ & $25 \%$ & 1.76 \\
\hline Until the behaviour no longer improves my emotion or the situation & $30 \%$ & $30 \%$ & - \\
\hline \multicolumn{4}{|l|}{ When do you stop engaging in worry in response to uncertainty? } \\
\hline After uncertainty resolves & $75 \%$ & $15 \%$ & $14.55^{* *}$ \\
\hline After I take action & $15 \%$ & $45 \%$ & $4.29 *$ \\
\hline When I distract myself & $25 \%$ & $20 \%$ & 0.14 \\
\hline When I get reassurance from others & $15 \%$ & $15 \%$ & - \\
\hline When I reassure myself & $10 \%$ & $20 \%$ & 0.78 \\
\hline
\end{tabular}

Note. Participants were asked to describe when they stop doing the main behaviours that they use in response to uncertainty (not including worry). Since worry is considered a specific behavioural response to uncertainty (Ladouceur et al., 2000; Rosen \& Knäuper, 2009) and is the cardinal symptom of GAD, people were separately asked when they stop worrying. Participants often offered more than one response, thus percentage totals do not add up to 100. As participants were not asked about each theme specifically, it is possible that a given theme applied to a person, although the participant did not spontaneously report it.

${ }^{*} p<.05, * * p<.01$. 
The results showed that $40 \%$ of people with GAD and $0 \%$ of the NPC group described engaging in behavioural responses to uncertainty until it is no longer possible to do so. Reasons for behaviours no longer being possible included: 1) when individuals "ran out" of people to seek reassurance from, e.g., “you get as many opinions as you can” and "once I've asked all the people I trust”; 2) when carrying out the behaviour became too physically or emotionally taxing, e.g., "when it becomes impossible to handle [continuing the behaviour]" and "when I'm pushed to the brink I have to make the decision"; and 3) when external constraints prevented the continuation of the behaviour(s), e.g., "when time is up.” As no participants in the NPC group reported responses consistent with the until it is no longer possible theme, reasons for behaviours ceasing to be possible in this group are not reported.

For the after I do it once or twice theme, 45\% of the non-psychiatric control group and $0 \%$ of the GAD group described engaging in behavioural responses to uncertainty a few times and then stopping. Examples of this theme were: "I do it once or twice and stop, and feel better”; "I just have a heuristic of like check it over once...I'm normally satisfied with any changes I've made and I figure I don’t need to do it again”; and “typically once I reassure myself I try not to think about it again. I move on.”

There was no difference in the number of people from the GAD versus NPC group who described responses consistent with the until I have a subjective feeling to stop theme. Some responses involved experiencing a felt sense to stop engaging in a behavioural response to uncertainty, for example, "when I get to that point internally” (GAD) and "a gut feeling” (NPC). Other responses consistent with this theme reflected the notion that individuals cease behavioural responses to uncertainty when they feel better. For instance "if I'm feeling better, like I'm in a more positive attitude” (GAD) and "once I feel I have made my best effort...I am happy and I 
stop” (NPC). Still other answers consistent with this theme reflected the notion of stopping uncertainty-related behaviours because of an internal feeling that one had enough information. For example: "it becomes too much information for my brain so I stop" (GAD) and "I've exhausted information” (NPC).

Some individuals in the GAD and NPC groups reported that they stop engaging in behavioural responses to uncertainty when the acts do not help their feelings or the situation. In some cases, individuals referred to reassurance seeking not providing a sense of increased certainty. For instance, “if they are giving me advice that I don’t find helpful or reassuring in any way” (GAD). In other cases, individuals described ceasing avoidance when it begins increasing rather than reducing distress, e.g., "the anxiety builds up" (GAD). Finally individuals described ceasing checking and information seeking behaviours when the acts no longer improve outcomes. For example, "when I reach a point and I say I can continue to do this, but it's always going to be the same outcome" (NPC).

Discontinuation factors (worry). Consistent with the hypothesis that more people with GAD than NPCs will continue behavioural responses to uncertainty until they are certain, many people with GAD (75\%) described answers that fit with the after uncertainty resolves theme, meaning that they would continue to worry about something until they knew the outcome with certainty. Examples of this theme were: "I would say I worry up until it’s resolved."; "Once the result is delivered.”; and “It’s constantly worry about things going wrong, and I won’t be ok until I'm actually there.” Significantly fewer NPCs (15\%) described responses in line with this theme, but of those who did, they described it in a qualitatively similar manner. For instance, "The worry doesn’t stop until the uncertainty goes away." and "It would just bother me until I resolve it.” 
For the after I take action theme, $45 \%$ of the non-psychiatric control group said that taking action leads to worry cessation. For instance, "So I find that I take some sort of action...you know you are doing something about it, you are not dwelling on it" and "finishing one step definitely calms me.” On the other hand, only 15\% of the GAD group described answers that fell into this theme, e.g., "once something gets done.” There were no noteworthy qualitative differences in responses consistent with the after I take action theme between groups.

A proportion (15\% - 25\%) of individuals in the GAD and NPC groups reported distraction, seeking reassurance from others and reassuring oneself as other factors that lead to worry cessation, with no quantitative or qualitative differences between groups. Within the when I distract myself theme, some people said that worry stops when something else arises that distracts them from the worry. For example, “...something else distracts me, but it’s like a welcomed distraction. Like I can’t deal with this right now, because there’s something else that's more important” (GAD). People also described actively seeking out a distraction from worry, e.g., "when I go do something else” (NPC). Sample responses from the when I get reassurance from others theme were: "the reassurance from others" (GAD) and "talking to my parents and family, if I'm very worried I'll just talk to them, and my mom is always like don't worry, you'll get a job, so there's that reassurance" (NPC). Examples of phrases consistent with the when I reassure myself theme were: "So I go over things to reassure myself like how I am prepared or how I could've overcome that negative consequence if it did happen” (GAD) and "then I reassure myself, think about it slowly” (NPC).

\section{Objective 4. Impairment Caused by Behavioural Responses to Uncertainty}

The fourth goal of the present study was to better understand whether behavioural responses to uncertainty are problematic for individuals with GAD or NPCs, and if so, in what ways. It was hypothesized that more individuals with GAD than NPCs would describe their 
uncertainty-related behaviours as problematic; and it was further suspected that relationship strain, work/school difficulty, or physical ailments would be ways in which behavioural responses to uncertainty were problematic. In line with expectations, the results showed that almost all individuals with GAD (93\%) said that their behavioural responses to uncertainty cause problems for them, whereas this was only true of one non-psychiatric control participant (5\%; $\chi^{2}$ $=25.32, p<0.01$ ). Table 12 provides a summary of the problems that people with GAD said behavioural responses to uncertainty cause them.

One of the most frequently described problems by people with GAD was feeling high levels of emotional and cognitive strain, e.g., "Just stressing myself out. Like mentally exhausting myself" and "it just makes me.... self-tortured." Another theme commonly found in the GAD group’s responses was work or school difficulties, for instance, "procrastination definitely does have a huge effect on my marks and that kind of starts more worrying.” People in the GAD group also discussed behaviours causing relationship strain, e.g., "my boyfriend will get fed up with me...he will reassure me, but eventually he will be like okay you have got to stop; this isn't anything to be worried about.” Individuals with GAD also said that uncertaintyrelated behaviours keep them from achieving work-life balance or engaging in pleasant activities. For example: "there is so much I can do in my life but I am just sort of like sitting in this avoidance bubble and not enjoying life to the max because of all the uncertainties that I am always thinking about.” Finally, some individuals in the GAD group reported problems relating to physical ailments, for instance, "it [anxiety related to reassurance seeking] affects me physically and I'm prone to headaches and migraines because of the tension so it affects me in a physical way as well.” 
Table 12

Impairment Associated with Behavioural Responses to Uncertainty

\begin{tabular}{|c|c|c|c|}
\hline Themes & $\begin{array}{c}\text { GAD } \\
(n=14)\end{array}$ & $\begin{array}{c}\text { NPC } \\
(n=19)\end{array}$ & $\chi^{2}$ \\
\hline Behavioural responses to uncertainty cause problems & $93 \%$ & $5 \%$ & $25.32 * *$ \\
\hline \multicolumn{4}{|l|}{ Types of problems caused: } \\
\hline Cognitive/emotional distress & $43 \%$ & $5 \%$ & $6.82^{* *}$ \\
\hline Work/School problems & $43 \%$ & $0 \%$ & $9.95 * *$ \\
\hline Relationship problems & $36 \%$ & $0 \%$ & $7.99 * *$ \\
\hline Lack of life balance / low engagement in pleasant activities & $29 \%$ & $0 \%$ & $6.18 *$ \\
\hline Physical ailments (e.g., headaches, muscle pain) & $21 \%$ & $0 \%$ & $4.48 *$ \\
\hline
\end{tabular}

Note. Participants were asked if the main behaviours they use in responses to uncertainty cause problems for them, and if so, what types of problems. Participants often offered more than one response, thus percentage totals do not add up to 100. As participants were not asked about each theme specifically, it is possible that a given theme applied to a person, although the participant did not spontaneously report it.

$n=14$ in the GAD group and 19 in the NPC group; unequal $n$ s were due to the question being added after data collection had commenced.

${ }^{*} p<.05, * * p<.01$. 


\section{Discussion}

The present study was designed to answer questions about behavioural responses to uncertainty in people with GAD compared to non-psychiatric control participants (NPCs). This study used a mixed qualitative and quantitative approach to address four research objectives: 1) what are the form and frequency of behavioural responses to uncertainty; 2) what function do behavioural responses to uncertainty serve; 3) what factors lead people to stop behavioural responses to uncertainty; and 4) are behavioural responses to uncertainty problematic, and if so, in what ways. The results pertaining to each research aim are described below and discussed in relation to GAD theory and treatment.

\section{Form and Frequency of Behavioural Responses to Uncertainty}

In response to an open-ended question, more people in the GAD than in the NPC group spontaneously described worrying, over-preparing, repeatedly checking things over, avoiding, distracting themselves, and doing nothing as part of their typical response to uncertainty. People with GAD also employed a greater number of different behaviours (e.g., checking, reassurance seeking) when uncertain than did NPCs. Conversely, more people in the NPC group than in the GAD group spontaneously reported problem solving as part of their typical response to uncertainty.

In response to a closed-ended list of behaviours, people with GAD reported using the following behaviours with the highest frequency over the past month: worrying, excessive checking, reassuring oneself, doubting decisions already made, over-preparing, overestimating the chance of negative events, and reassurance seeking. People with GAD used these and most other behaviours that were assessed significantly more often than did NPCs. On the other hand, people in the NPC group reported using the following behaviours with the highest frequency: reassuring oneself, accepting/making the best of uncertainty, problem solving, replacing negative 
thoughts with positive or neutral thoughts, and information seeking. It seems that people with GAD and NPCs may have different "go to" behaviours that they engage in when uncertain. Overall, people in both groups endorsed using all of the behaviours assessed to some extent; however, people with GAD used most of the behaviours assessed significantly more frequently than NPCs.

The behavioural responses to uncertainty that people with GAD reported using frequently may be associated with problematic outcomes. For example, worrying and overestimating the likelihood of negative events have been linked to the maintenance of negative affect (e.g., Michl, McLaughlin, Shepherd, \& Nolen-Hoeksema, 2013; Pieper, Brosschot, van der Leeden, \& Thayer, 2010). In addition, doubting oneself has been associated with a sense of reduced selfesteem (Greenberg, 1999; Joiner, Alfano, \& Metalsky, 1992). Moreover, excessive reassurance seeking has been related to strained interpersonal relationships (Heerey \& Kring, 2007; Starr \& Davila, 2008). On the other hand, some of the strategies that NPCs reported using most often when uncertain resemble techniques that are used in the treatment of excessive worry (Ballenger et al., 2001). For instance, problem solving is used in several evidence-based GAD treatments (e.g., Brown et al., 2001; Dugas \& Robichaud, 2007; Zinbarg et al., 2006) and accepting uncertainty parallels what is taught to clients in mindfulness-based CBT approaches (e.g., Roemer \& Orsillo, 2007). In sum, the typical responses to uncertainty used by people with GAD may contribute to other problems, further fueling worry, whereas some of the typical responses to uncertainty used by NPCs may actually improve anxiety.

It is of note that when asked about their typical responses to being uncertain in an openended manner, individuals with GAD described problem solving (i.e., generating and weighing potential solutions and executing solutions; see D’Zurilla \& Nezu, 2010) significantly less often 
than did non-psychiatric control participants. However, when asked to report the number of times they engaged in problem solving over the past month, without being given a definition of problem solving, people with GAD and non-psychiatric control participants reported problem solving with comparable frequency. Research has shown that people with GAD consider worrying to be an important problem solving activity (see Davey, 1994; Davey, Tallis, \& Capuzzo, 1996; Wells \& Carter, 2001), even though worrying does not actually result in effective solution generation or implementation (Stöber, 1998). Thus, one reason why individuals with GAD may have reported problem solving as often as non-psychiatric controls when asked to report the frequency of problem solving over the past month may be because people with GAD considered worrying to be problem solving. On the other hand, when people described their actions when they were uncertain, problem solving would have been coded only if it was consistent with an objective definition, potentially leading to the discrepancy between the findings about problem solving on the open-ended versus closed-ended question.

In addition, it is of note that the shortened version of the IUS (the IU-12; Carleton et al., 2007) has been found to comprise two factors: prospective anxiety and inhibitory anxiety (Carleton et al., 2007). Carleton and colleagues posit that prospective anxiety reflects the “cognitively focused dimension of IU” (e.g., "unforeseen events upset me greatly”) and that inhibitory anxiety reflects the "behaviourally focused dimension of IU" (e.g., "the smallest doubt can stop me from acting”; described in Carleton et al., 2012, McEvoy \& Mahoney, 2011). This conceptualization of the behavioural dimension of IU as "inhibitory" may suggest that behavioural responses to IU fall into the "avoidance" spectrum. Based on this, it was of interest to conduct a posthoc analysis to compare whether avoidance or approach strategies were used more often by individuals with GAD. The results showed that there were no significant 
differences in the frequency of avoidance versus approach behaviours in the GAD group, $t(27)=$ $1.42, p=.17$, or the NPC group, $t(27)=0.33, p=.74$. Thus, the present data suggest that there are not systematic differences in the frequency with which individuals with GAD or NPCs avoid or approach uncertainty; it seems that people engage in both strategies.

\section{Functions of Behavioural Responses to Uncertainty}

The main functions of people's behavioural responses to uncertainty were to reduce distress, increase certainty, feel prepared for the worst, attain the best possible outcomes, and elicit support from others. There were no differences between groups in the number of people who reported each function, with one exception. More people in the GAD versus NPC group said that their behavioural responses to uncertainty served the function of helping them to feel prepared for the worst. Responses consistent with this theme reflected the notion that individuals with GAD would rather engage in behaviours to prepare emotionally or practically for a potential negative outcome than be caught off guard by a negative event. The specific behaviours that people with GAD said prepared them for the worst were worrying and overestimating the probability of negative events.

This finding is consistent with Newman and Llera’s (2011) Contrast Avoidance Theory of GAD, proposing that people with GAD worry to maintain a constant negative emotional state, avoiding sudden changes from a positive or neutral state to a negative state. The findings of several studies are in line with the main tenets of the Contrast Avoidance Theory (e.g., Hofmann et al., 2005; Lyonfields et al., 1995; Peasley-Miklus \& Vrana, 2000; Vrana \& Lang, 1990), and recent studies have provided additional empirical support (Andor et al., 2008; Llera \& Newman, 2010, 2014; Stapinski et al., 2010). The present results are similarly consistent with the notion that people with GAD employ worry and overestimate the probability of negative events to feel prepared for the worst (Sibrava \& Borkovec, 2006). 
It is of note that although significantly more people with GAD than non-psychiatric control participants said that their behaviours helped them feel prepared for the worst, the absolute number of people with GAD who described this was low (35\%) given the research highlighting the importance of feeling prepared for the worst in GAD (see Newman \& Llera, 2011). This may be because not all individuals with GAD are aware that their behaviours help them feel prepared for the worst. It is also possible that people are aware that worrying and overestimating the probability of negative events make them feel prepared for the worst, but do not spontaneously consider this when asked about the function of their other main behavioural responses to uncertainty. Another reason why the number of people with GAD reporting the feel prepared for the worst could have been low is because of the present study's methodology, which asked people to report on what they get out of the main one or two behaviours that they use when uncertain. It is possible that feeling prepared for the worst is a key function of worry and overestimating the probability of negative events, however, not everyone in the GAD group reported on what they get out of these behaviours. The implications of the present methodology are considered further in the Limitations section, and future research could expand on the present findings by examining the specific functions of each different behavioural response to uncertainty.

The high proportion of people in both groups who reported engaging in behaviours in order to reduce distress and increase certainty is in line with research suggesting that most people have some level of discomfort with uncertainty (see Inglis, 2000 for a review). Indeed, most people prefer to be certain than uncertain (Curley et al., 1986; Epstein, 1999; Fox \& Tversky, 1995; Montesano, \& Giovannoni, 1996), and are motivated to increase certainty (e.g., Berger \& Calabrese, 1975; Brashers et al., 2000; Ellsberg, 1961; Kramer, 1999; Lipshitz \& 
Strauss, 1997; Rosen et al., 2007). In fact, Kalma (1986) argued that uncertainty reduction is a fundamental biological requirement of all species in order to survive and thrive, and neurophysiological studies have shown that animals have an innate tendency to seek out information to reduce uncertainty (see Panksepp, 1998). These findings are also consistent with research showing that one key function of both reassurance seeking and checking is to reduce anxiety in individuals with OCD or major depressive disorder (MDD; Parrish \& Radomsky, 2010). In sum, behavioural responses to uncertainty may be aimed at increasing certainty and reducing distress, regardless of whether or not psychopathology is present.

A qualitative difference that emerged between people with GAD and people without a psychological disorder was that only people with GAD described the reduction in distress from behaviours when uncertain as transient or partial, which may help explain the frequent engagement in behavioural responses to uncertainty in this population. In addition, most people with GAD framed their responses in negative terms (e.g., to reduce stress), whereas most nonpsychiatric control participants described theirs in positive terms (e.g., to increase confidence). Given that individuals with GAD are known to have higher IU than NPCs (Dugas et al., 2004; Dugas et al., 1998; Gentes, \& Ruscio, 2011), it is possible that this qualitative finding reflects a more negative attitude toward uncertainty in the GAD group.

On the flipside of wanting to feel prepared for the worst, individuals with GAD and NPCs reported that their behaviours served to help them attain the best possible outcomes in situations. For instance, some said that checking things over helped to ensure achievement in school, and others said that over-preparing helped guarantee that plans or projects went well. Although there was no quantitative difference between groups in the number of responses that were consistent with this theme, there was a qualitative difference. The necessity of engaging in 
behaviours when uncertain in order to optimize outcomes was evident in $50 \%$ of the responses of the GAD group (i.e., "I need to get all the information”), but was not apparent in the NPC responses. This finding is in line with research showing that people with GAD report beliefs about the usefulness of engaging in worry (e.g., worry motives me, worry protects me) to a greater extent than NPCs (Davey et al., 1996; Freeston et al., 1994; Wells, 2005). The current results extend prior research by suggesting that people with GAD may not only hold positive beliefs about the usefulness of worrying, but also about the usefulness of other strategies, such as overpreparing or excessive checking. It may be useful to broaden the notion of positive beliefs about worry, to positive beliefs about various behavioural responses to uncertainty.

Finally, a small proportion of people in both groups said that they engage in reassurance seeking in response to uncertainty in order to elicit the support of others, and there were no differences between groups. The result is in keeping with research suggesting that having a person to talk to when uncertain can help individuals better manage stress (see Brashers et al., 2004; Rosen \& Knäuper, 2009). It is also consistent with Parrish and Radomsky’s (2010) finding that one of the functions of reassurance seeking in people with OCD, MDD, or no psychological disorder was to receive affection. Although reassurance seeking may produce some benefits, it is of note that excessive reassurance seeking is paradoxically associated with interpersonal rejection (see Haeffel, Voelz, \& Joiner, 2007; Lemay \& Cannon, 2012; Starr \& Davila, 2008). Thus, although the function of reassurance seeking in individuals with GAD appears normative, the frequency with which people with GAD seek reassurance may led to inadvertent negative effects on relationships. Currently much of the research on excessive reassurance seeking relates to individuals with MDD (e.g., Joiner et al., 1999; Starr \& Davila, 
2008), and more research on the effects of excessive reassurance seeking in GAD samples would be informative.

\section{Factors that Lead to the Discontinuation of Behavioural Responses to Uncertainty}

The current study explored factors that lead to the cessation of behavioural responses to uncertainty including worry. One cessation factor for behaviours in general, including worry, was the uncertain situation being resolved (i.e., the person is no longer uncertain). This theme was described by significantly more people in the GAD versus NPC group. Another finding was that the behaviours stop when they are no longer possible, typically because of situational constraints (e.g., running out of time); this theme was expressed by significantly more people with GAD compared to NPCs. Both of these themes highlight that it is difficult for individuals with GAD to tolerate not knowing and that they perseverate in their attempts to achieve certainty.

The current findings are consistent with research showing that although people with GAD and NPCs engage in information seeking when uncertain, high worriers persist with information seeking for longer than others, slowing decision-making and task completion (Ladouceur et al., 1997; Metzger et al., 1990; Tallis et al., 1991). Indeed, the current findings suggest that people with GAD may continue with a range of behavioural responses to uncertainty, including seeking reassurance, reassuring themselves, overpreparing, or distracting themselves for longer than NPCs.

On the other side of the coin, the results showed that significantly more people in the NPC versus GAD group said that they stop behavioural responses to uncertainty after engaging in them once or twice. This is consistent with the present study's other finding that NPCs engage in behavioural responses to uncertainty significantly less frequently than people with GAD. It is possible that non-psychiatric control participants engage in behavioural reactions to uncertainty 
just once or twice because they require less of a sense of certainty than do people with GAD. Another possibility is that relative to people with GAD, people without a psychiatric diagnosis engage in behaviours once or twice because they choose behaviours that are more effective in providing certainty or are better suited to a particular situation. For instance, seeking reassurance about how one did on a test from another person who wrote the same test would likely provide a greater sense of certainty than seeking reassurance from someone who did not write the test.

Another factor that led to relief from worry was taking action, and more people in the NPC than GAD group described this theme. This dovetails with the finding that most NPCs described problem solving as a typical response to uncertainty when asked about this in an openended manner, which was true of significantly fewer people with GAD. Research has similarly shown that the ability to execute solutions to problems is associated with reduced anxiety, stress, and worry (see Chang, D’Zurilla, \& Sanna, 2004; D’Zurilla \& Nezu, 2010; Ladouceur et al., 2000). The current result also maps onto research suggesting that taking action is the part of the problem solving process that high worriers have the most difficulty with (Borkovec, 1985; Ladouceur, Blais, Freeston, \& Dugas, 1998). Indeed, although worriers do not lack knowledge of problem solving skills, they tend to have negative views of problems that can interfere with acting on their knowledge (e.g., Dugas et al., 1995).

In terms of common cessation factors of behavioural responses to uncertainty across groups, both groups described stopping behaviours when they felt a subjective sense that they had done enough. Participant responses consistent with this theme involve stopping a behaviour following a "gut feeling" that it had been done enough, and stopping a behaviour after "feeling better.” The former type of response is consistent with Wells and Carter's (2001) theorizing about positive beliefs about worry, suggesting that people terminate worry when they have an 
internal "felt sense" that they have considered most possibilities. The notion of having a subjective sense of when to stop is also consistent with findings from the animal literature implying that animals each have varying needs for information, and will cease information seeking when their need is met (e.g., Glanzer, 1958; Donohew, Tipton, \& Clarke, 1973). The latter type of response, to stop when one feels better, is in line with the Mood-As-Input Theory of Worry (Davey, 1994; Davey, Eldridge, Drost, \& MacDonald, 2007; Davey, Startup, MacDonald, Jenkins \& Patterson, 2005), positing that people are signalled by their mood to cease or persist with worrying. Research has shown that people continue to worry when their mood is more negative, and disengage from worry when their mood is more positive (Davey et al., 2005, 2007; Startup \& Davey, 2003). MacDonald and Davey (2005) have extended the Mood-As-Input Theory of Worry to perseverative checking, and have found that individuals also persist with checking for longer when in a negative mood. The current data also suggest that people may use internal signals, such as a felt sense or their mood, to guide the cessation of worry and other behavioural responses to uncertainty.

In addition, the same proportion of individuals in both groups (30\%) said that they stop using behavioural responses to uncertainty when it no longer improves their emotions or the situation. This finding is considered in light of the finding that people with GAD also said that they continue some behavioural response as long as they are uncertain. Together, the results suggest that people with GAD may replace one behaviour that is not producing desired effects with another. On the other hand, most non-psychiatric control participants did not describe being certain as a requirement to stop engaging in behavioural responses to uncertainty; thus, it is not suspected that non-psychiatric controls replace one behaviour with another when a behaviour no longer improves their emotions. This is also theorized based on research suggesting that 
nonclinical samples cease behavioural responses to uncertainty when it becomes clear that uncertainty cannot be avoided or reduced (Babrow \& Klien, 2001; Lipshitz \& Strauss, 1997; Mischel, 1990). The question of whether individuals with GAD switch uncertainty-management strategies when one behaviour is not having beneficial effects was not directly examined in the present study, but instead, inferred based on the pattern of results. Thus, future research should aim to clarify this potential difference between people with GAD and NPCs.

A small proportion of both the GAD and NPC groups expressed that distracting themselves, reassuring themselves, and seeking reassurance were all behaviours that stop worry. Given the present study's finding that people with GAD engage in these behaviours significantly more frequently than NPCs, it would be informative for future research to clarify how long behaviours such as distraction and reassurance-seeking quell worry for in people with GAD versus NPCs. Findings from the present study suggest that engaging in such behaviours may promote worry cessation in both groups. However, given the present data on how frequently worry arises in people with GAD relative to individuals without a psychological disorder, it is possible that the effects of strategies used to cease worry may be shorter-lived or ineffective in people with GAD compared to non-psychiatric control participants.

\section{Impairment Caused by Behavioural Responses to Uncertainty}

The results showed that behavioural responses to uncertainty caused problems for almost all individuals with GAD, whereas this was true of only one NPC. This finding highlights an important difference in the consequences of behavioural responses to uncertainty in people with GAD versus NPCs. The problems caused by behavioural responses to uncertainty were: cognitive and emotional distress, work and school problems, relationship problems, lack of pleasure and life balance, and physical ailments. In other words, people with GAD said that their behavioural responses to uncertainty caused problems for them across various areas of life. 
These findings are in line with studies showing the extensive negative effects of pathological worry on functioning. Studies have shown that GAD is associated with a comparable level of impairment as MDD in terms emotional, occupational, and physical health consequences (see Dugas, Anderson, Deschenes, \& Donegan, 2010, and Hoffman, Dukes, \& Wittchen, 2008 for reviews). In addition, studies have demonstrated a link between GAD and relationship problems (Borkovec et al., 2004; Eng \& Heimberg, 2006). The present results suggest that the relationship difficulties of people with GAD may be due, in part, to behavioural responses to uncertainty (e.g., reassurance seeking), which may place considerable strain on significant others.

The current results provide novel evidence that responses to uncertainty are problematic from the perspective of people with GAD. Although this has been described previously on theoretical grounds (Dugas \& Robichaud, 2007), it is now supported from an empirical standpoint. The distressing nature of behavioural responses to uncertainty emphasizes the usefulness of considering such behaviours in conceptual models of GAD, and in treatment approaches.

\section{Implications}

The present study offers several new pieces of evidence supporting the theory that people with GAD are intolerant of uncertainty (Dugas et al., 1998, 2007; Dugas \& Robichaud, 2007). To summarize, the current study found that compared to non-psychiatric control participants, people with GAD engage in a greater number of behaviours when uncertain, do so more frequently, and report that these behaviours are problematic. The current study also provided data that directly supports the link between IU and worry, by showing that people with GAD: spontaneously describe worry as part of their typical response to uncertainty, use worry more

frequently than any other behavioural response to uncertainty, and do not stop worrying until 
they are certain. Taken together, the present findings strengthen the main underlying theoretical assumption of the IU model of GAD, that difficulty accepting uncertainty is a key factor underlying excessive worry.

The current findings are in line with proposals to add behaviours to the GAD diagnostic criteria in DSM-5 (Andrews et al., 2010; Andrews \& Hobbs, 2010; Starcevic \& Portman, 2013). Specifically, the present study provided support for the notion that people with GAD seek reassurance, overprepare, procrastinate, and avoid activities with uncertain outcomes more often than do NPCs. These were precisely the behaviours that were considered for inclusion as symptoms demarcating GAD in DSM-5, although the behaviours were not ultimately added to the diagnostic criteria due to a lack of adequate empirical support (see Andrews et al., 2010). The current study not only supports continued investigation of behaviours that are elevated in people with GAD compared to people without a psychiatric diagnosis, but also suggests that the diverse behaviours of individuals with GAD may be tied together by the common thread of IU.

One of the aims of the present study was to learn more about how the behaviours of people with GAD and NPCs differ. Both the GAD and NPC groups reported reassuring themselves and seeking reassurance as part of their typical response to uncertainty; however, NPCs engaged in these behaviours much less frequently than did people with GAD. Indeed, the frequency findings showed that although NPCs engaged in most of the same behavioural responses to uncertainty as the GAD group, the NPC group engaged in such behaviours much less often. These results are consistent with previous research that has conceptualized worry on a continuum, suggesting that people with GAD and NPCs worry about the same things, except that people with GAD worry more frequently (Hazlett-Stevens, Ullman, \& Craske, 2004; Olatunji, Broman-Fulks, Bergman, Green, \& Zlomke, 2010; Ruscio, Borkovec, \& Ruscio, 2001). The 
current findings extend prior research by suggesting that numerous behavioural responses to uncertainty, including worry, might be conceptualized on a continuum, rather than pathological per se.

Although it was generally found that people with GAD engaged in behaviours when uncertain more frequently than NPCs, there were some behavioural responses to uncertainty that both groups reported engaging in with comparable frequency when asked in a closed-ended manner. These included: excessive information seeking, problem solving, making decisions quickly without weighing the options, thought replacement, and not fully committing to uncertain projects/relationships. Some of these results are in line with research in nonclinical literatures showing that seeking information and problem solving can be adaptive ways to deal with uncertainty (Brashers et al., 2000; Cooney \& Lang, 2007; Heyman, Henrikson, \& Maughan, 1998; Kramer, 1999; Lipshitz \& Strauss, 1997). These results also suggest that some strategies that might be considered unhelpful, such as thought replacement, may in fact be normative.

The lack of difference between groups in acting without thinking through the consequences when uncertain was somewhat surprising given prior research. Pawluk and Koerner (2013) found that people with GAD have a tendency to act quickly in response to negative affect (i.e., negative urgency) compared to people lower in worry; thus, people with GAD would be expected to act quickly when uncertain more often than NPCs. The lack of difference between groups may be an artifact of the low base rate of this behaviour in the present study (i.e., 0-4 times per month). Future research should seek to better understand negative urgency as a potential behavioural response to uncertainty in people with GAD, and the degree to which it characterizes only a subset of people with GAD. 
In addition to a difference in how frequently people with GAD engage in behavioural responses to uncertainty compared to NPCs, the current results also suggest that there may be differences in the factors that cue the cessation of behavioural responses to uncertainty between people with GAD and non-psychiatric control participants. The results suggested that people with GAD may have difficulty disengaging from their behavioural responses to uncertainty, as the GAD group described continuing behavioural responses to uncertainty until they were certain, or until the behaviours were no longer possible to perform. On the other hand, most individuals without a psychological disorder did not describe being certain as a factor that led to the cessation of their behaviours, but rather, most commonly described stopping after having performed a behaviour a few times. Thus, although more research is needed, it is possible that the ease with which one can disengage from a behavioural response to uncertainty may be a factor that distinguishes healthy from maladaptive behaviours. Indeed, research on OCD has shown that compared to non-psychiatric control participants, people with OCD have more difficulty stopping behaviours that they use to ameliorate anxiety (e.g., washing in people with contamination fears; e.g., Hinds, Woody, Van Ameringen, Schmidt, \& Szechtman, 2012; Szechtman \& Woody, 2004). Some studies suggest that difficulty stopping compulsive behaviours in OCD is perpetuated by internal rules to continue and elevated evidence requirements (e.g., Cougle, Goetz, Fitch, \& Hawkins, 2011; Wahl, Salkovskis, \& Cotter, 2008). Examining whether difficulty disengaging from behaviours distinguishes people with GAD from people without a psychological disorder using experimental methods that have been used in OCD research is a direction for future study.

Prior research has emphasized that strategies that increase a person's ability to accept uncertainty are helpful in the treatment of GAD (see Lohr, Olatunji, \& Sawchuk, 2007). The 
current results are consistent with this suggestion, and support the use of treatment approaches that target IU in people with GAD. The uncertainty recognition and behavioural exposure module outlined in CBT-IU for GAD (Dugas et al., 2010; Dugas \& Robichaud, 2007) is theoretically designed to improve people’s tolerance for uncertainty by having them gradually approach situations with uncertain outcomes and reducing excessive behavioural responses to uncertainty (e.g., refraining from excessive reassurance seeking). The current results provide information about what behavioural patterns may be important to target in GAD treatment.

In addition, the results showed that people with GAD engage in behavioural responses to uncertainty more frequently than do non-psychiatric control participants, suggesting that one important aspect of treatments targeting IU may be to help people reduce the number of times they carry out behaviours in response to uncertainty. The findings also point to the possibility that people with GAD have difficulty disengaging from uncertainty-related behaviours, and as such, may benefit from reducing the duration of their behavioural responses to uncertainty in treatment. In sum, gradually planning to reduce the number of times one engages in a behavioural response to uncertainty, or the duration of behavioural responses to uncertainty may be useful treatment aims.

The current study also indicates that NPCs may problem solve when uncertain more often than people with GAD. Further, more NPCs than people with GAD described taking action as a factor that stopped worry. This is in line with research suggesting that executing solutions to problems is a key way to stop the worry cycle (see Chang et al., 2004; D’Zurilla \& Nezu, 2010). Currently, providing psychoeducation about the importance of solving problems in order to improve worry is an aspect of some CBTs for GAD, including CBT-IU (Dugas \& Robichaud, 2007). Given this study’s findings, the use of interventions for GAD that facilitate problem 
solving when uncertain is indicated (e.g., Dugas \& Robichaud, 2007; Zinbarg et al., 2006). In sum, helping people with GAD to take actions that address their concerns as one way to stop worrying is important.

The present findings from non-psychiatric control participants provide a sense of what clinicians might expect as “normal” responses to uncertainty following GAD treatment. The results showed that even people with no psychological disorder engage in strategies to manage uncertainty that were measured in the present study. In addition, the main functions of behavioural responses to uncertainty in NPCs were to increase certainty and reduce distress, suggesting that on the whole, non-psychiatric control participants prefer to be certain and feel at least some level of discomfort with uncertainty. Thus, people with GAD can be expected to continue to engage in some IU-related behaviours, and for similar reasons as they did at pretreatment; however, they might be expected to engage in such behaviours less frequently. Other potential indicators that individuals with GAD are exhibiting more healthy use of behaviours when uncertain may be that they can more easily disengage from such behaviours, and do so before they are certain. A key difference between people with GAD and NPCs was that people with GAD said that their behavioural responses to uncertainty were problematic for them, whereas NPCs did not. Thus, an important treatment target may be to help people with GAD reduce their behavioural responses to uncertainty to a level that no longer interferes with their lives.

Finally, the results showed that people with GAD describe lacking enjoyment and life balance due to their behavioural responses to uncertainty. This finding is in line with the notion that people with GAD excessively consider future threats at the expense of present-moment pleasures (Borkovec, 2002). One clinical implication of this result is that people with GAD may 
benefit from the incorporation of mindfulness strategies in traditional CBT approaches (Roemer \& Orsillo, 2007).

\section{Limitations}

The present study had several strengths, including using a multimethod approach to explore novel research questions about behaviours related to IU, which yielded new insights about how IU manifests in people with GAD. The current study also used a comparison group of non-psychiatric control participants, providing information on normative aspects of behavioural responses to uncertainty. In addition, a semistructured interview was administered to determine eligibility for the present study; thus people with principal diagnoses more impairing than GAD were excluded, and the data relate to people with predominant GAD. Finally, the present study took several steps to ensure that the method for interpreting qualitative data was rigorous, including the creation of a detailed codebook and the use of two independent raters. This approach resulted in coding with excellent interrater reliability.

Some of the limitations of the present study are shared with those described in detail in Study 1a of this dissertation, since Study 1a and Study 1b each present data from the same large research investigation (see Study 1a, p. 93-95 for a discussion of limitations). A limitation specific to the present study was that individuals were not asked how often they actually feel uncertain; thus, although information about the frequency of IU-related behaviours was gathered, what remains unknown is the proportion of the time that people feel uncertain and engage in behavioural responses to uncertainty. Since the base rate of feeling uncertain was not assessed in the present study, it is unknown if people with GAD feel uncertain more often than individuals without a psychological disorder, and correspondingly engage in behavioural responses to uncertainty more frequently; or alternatively, whether people with GAD and non-psychiatric control participants feel uncertain with comparable frequency, and yet individuals with GAD 
engage in behavioural responses to uncertainty more often. Thus, future research investigating the base rate of feeling uncertain in people with and without GAD would extend the present findings.

Another consideration when interpreting the results of the current study is that the nonpsychiatric control group was asked to answer questions about their behavioural responses to uncertainty, such as the function of and problems caused by their IU-related behaviours, despite engaging in these behaviours with relatively low frequency compared to the GAD group. Thus, the non-psychiatric control group had a relatively "small sample" of instances of these behaviours, and it is possible that their responses to questions about IU-related behaviours were less stable relative to the responses of people who engage in these behaviours more often, and thus have more examples of the behaviours to draw on. As such, the responses of the nonpsychiatric control group to questions about the function, discontinuation factors, and problems associated with behavioural responses to uncertainty should be interpreted cautiously and replicated in future research.

Another limitation of this study was that the functions and cessation factors of behavioural responses to uncertainty were discussed with participants as they pertained to each participants' most frequently-used idiographic behavioural responses to uncertainty. As a result, the data on functions and cessation factors of behavioural responses to uncertainty are in reference to a mix of different behaviours (e.g., reassurance-seeking, avoidance). In other words, participants described the function and cessation factors of their most commonly used behavioural responses to being uncertain, but this could vary across participants; thus, the present study does not provide information on functions or cessation factors associated with specific behaviours. This method may have influenced the present study's results because the 
whole sample did not report on each behaviour, potentially limiting the power to detect differences between the GAD and non-psychiatric control groups on specific behaviours. Future research comparing the function and cessation factors associated with specific behavioural responses to uncertainty may help to further elucidate differences between people with GAD and comparison groups.

\section{Future Directions}

Its limitations notwithstanding, the present study unearthed several novel insights from the perspectives of people with GAD and non-psychiatric control participants about behaviours that they engage in when they are uncertain. The rich data from the current study point to several future research directions, some of which have been highlighted throughout this thesis and some of which are described below.

Behavioural responses to uncertainty across diagnostic categories. The current study was the first to ask about the function, discontinuation factors, and problems caused by behavioural responses to uncertainty in people with GAD relative to a non-psychiatric control group. When a line of research is novel, as was the case with the present study, it is recommended that researchers take a stepped approach, starting with studies that are less timeand resource-intensive before moving to more involved methods (e.g., Clark-Carter, 2004). From this perspective, comparing people with GAD to one comparison group was an appropriate first step. Indeed, the present study demonstrated that individuals with GAD or no disorder can provide rich information about their behaviours when uncertainty in response to open-ended questions. Given that IU is elevated across a range of psychiatric difficulties (e.g., Carleton et al., 2012; Gentes \& Ruscio, 2011; Sternheim et al., 2011), an important extension of the current work is to examine differences in behavioural aspects of IU between people with GAD and people with other forms of psychopathology. Indeed, although research measuring IU with self- 
report assessments, such as the IUS and its short form the IU-12 has shown that individuals with different anxiety disorders or depression show similar levels of IU (e.g., Carleton et al., 2012; Holaway et al., 2006), it is possible that people with different disorders express IU in different ways - questions that may be explored and tested using qualitative or mixed methods approaches. Knowing whether there are differences in the scope or frequency of behavioural responses to uncertainty across diagnostic groups may provide information that helps to distinguish underlying processes in people with GAD versus other psychological difficulties, and may assist in identifying behavioural patterns that are unique to GAD, potentially helping to distinguish people with GAD from people with other diagnoses.

Measuring behavioural responses to uncertainty. The current self-report measures of IU in the GAD literature assess only a few behavioural responses to uncertainty (see Buhr \& Dugas, 2002), and yet the present data showed that there are numerous behaviours that individuals with GAD and NPCs employ when they are uncertain. This discrepancy highlights a need to more thoroughly measure behavioural responses to IU. In particular, measures that include behavioural manifestations of IU (e.g., the IUS, the IU-12; Buhr \& Dugas, 2002; Carleton et al., 2007) may benefit from expanding to include both approach and avoidance strategies; currently these measures include only a few avoidance behaviours (e.g., "I must get away from all uncertain situations”). It may be particularly useful to include behaviours that were identified as frequently used by the GAD group, such as overpreparing and repeated checking. It may also be important for future studies to expand on the present study's investigation of the frequency of behavioural responses to uncertainty, to include factors such as how difficult it is to disengage from behaviours, and the duration of behaviours. This might be done by asking people to keep a diary of the behaviours they use in response to uncertainty over 
the course of a week, and asking people to answer questions about behaviours when they arise, such as rating how difficult it is to stop a behaviour, and the duration of a behaviour. Finally, the literature on behavioural responses to uncertainty in GAD would be strengthened by the use of additional methods to investigate behaviours. For example, experiments that involve manipulating feelings of uncertainty and then assessing the degree to which people may engage in approach (e.g., seeking information) or avoidance (e.g., distracting oneself) behaviours in response to those feelings would extend and verify the present findings.

What makes some behavioural responses to uncertainty adaptive? Individuals in both the GAD and NPC groups mentioned that several behavioural responses to uncertainty actually allow them to stop worrying. These strategies were: distracting oneself, reassuring oneself, and seeking reassurance from others. Given these findings, it would be helpful for future research to clarify when strategies such as distraction and reassurance seeking are helpful for disengaging from states such as worry, and when such strategies become maladaptive. Indeed, the "line" between helpful and unhelpful behaviours is not yet clear. Several evidencebased treatments such as DBT (Linehan, 2015) and behavioural activation (Greenberger \& Padesky, 1995) even prescribe techniques that resemble distraction in that they encourage disengagement from distressing emotions or negative thought patterns through involvement in other activities (e.g., Dimidjian et al., 2006; Swales, 2009). It might be hypothesized that using strategies like distraction, reassuring oneself, and seeking reassurance may be helpful when done infrequently and for short periods of time, but may become unhelpful if done often and for prolonged periods of time. Future research clarifying this issue would provide important information on the adaptive versus maladaptive use of strategies such as distraction, which could guide clinical practice and perhaps aid in the assessment of behavioural manifestations of IU. 


\section{Summary and Conclusion}

The present study compared behavioural responses to uncertainty in people with GAD to people without a psychological disorder using a mixed qualitative and quantitative approach. People with GAD and non-psychiatric control participants reported engaging in similar behavioural responses to uncertainty, most of which could be categorized as approach or avoidance strategies. However, compared to non-psychiatric control participants, people with GAD used a greater range of behaviours when uncertain, and did so more frequently. The behavioural responses to uncertainty that people GAD spontaneously described using when uncertain included: worrying, overpreparing, repeated checking, avoiding, distracting, and doing nothing. The function of behavioural responses to uncertainty was similar for people with and without GAD; however, several people with GAD described short-lived or partial reductions in distress from behaviours. Also, more people in the GAD than NPC group described preparing for the worst as a function of their behaviours. Moreover, people with GAD said that they engaged in behavioural responses to uncertainty until the unknown situation was resolved, or it was no longer possible to do so, and reported that their behavioural responses to uncertainty caused problems for them; whereas the same was not true of non-psychiatric control participants. Overall, the present study provides novel support for Dugas et al.'s (1998) IU Theory of GAD and suggests that behavioural responses may be an important aspect of IU. The results also highlight the potential utility of targeting behavioural manifestations of uncertainty in GAD treatment in order to improve IU and reduce the impairment associated with GAD. The present study pointed to a number of future research directions including exploring behavioural responses to uncertainty across various diagnostic groups and developing measures of behavioural manifestations of IU. 


\section{Study 2: The Effects of Uncertainty Exposure on GAD-Relevant Symptoms and Processes}

\section{Introduction}

Generalized anxiety disorder (GAD) is a common disorder characterized by excessive, uncontrollable worry across several life domains such as work and school (APA, 2013). Like people with other anxiety disorders, people with GAD engage in considerable avoidance of situations that they perceive as threatening (see Wenzel, 2013). Cognitive behavioural theories propose that avoidance maintains anxiety by preventing people from learning that their beliefs about feared outcomes are inflated and possibly inaccurate (e.g., Hofmann 2008). A highly effective approach for reducing anxiety is counteracting the tendency to avoid, so that new learning about feared situations and one's ability to cope with them can occur (e.g., Ougrin, 2011). One strategy for counteracting avoidance is exposure, which refers to gradually and repeatedly approaching a feared object or situation with the intention of activating anxiety, and remaining in contact with the feared object or situation until one's anxiety reduces (e.g., Abramowitz et al., 2011; Wenzel, 2013), or until new learning occurs about how threatening the feared object or situation is (see Craske et al., 2014). Another strategy that targets avoidance is the behavioural experiment, which involves identifying a specific prediction (e.g., "I won't be able to handle the situation”), devising a plan to test the prediction, which usually requires approaching a feared object/situation, and then reappraising the original prediction after the test has been carried out (Bennett-Levy et al., 2004). Research to date suggests that exposure and behavioural experiments produce significant, large improvements in anxiety (see McMillan \& Lee, 2010 for a review). There is some evidence that behavioural experiments improve anxiety to a greater extent than exposure alone, although this has not been a consistent finding across studies or across measures within studies (see McMillan \& Lee, 2010 for a review). 
GAD is a complex disorder in that individuals' fears do not center on a specific theme, as they do in most other anxiety disorders (e.g., negative evaluation in social anxiety disorder, uncomfortable physical sensations in panic disorder). Individuals with GAD have concerns that cross several domains, such as family, work competence, or finances; and these worries typically shift over time (see Dugas, Freeston et al., 1998; Roemer et al., 1997). Accordingly, several models of GAD have been put forth in the literature conceptualizing the core underlying fears that may explain the development and maintenance of diffuse worries, and corresponding treatments have been developed (see Behar et al., 2009).

One theory proposed to explain the varied and fluctuating concerns of individuals with GAD is the Intolerance of Uncertainty (IU) Model of GAD (Dugas et al., 1998). This theory conceptualizes IU - a set of negative beliefs about uncertainty and its implications - as the underlying factor that leads to and maintains pathological worry and anxiety, even when the worries appear to be very diverse on the surface (Dugas et al., 1998). Individuals with GAD score higher on the Intolerance of Uncertainty Scale (IUS; Buhr \& Dugas, 2002) compared to nonclinical controls (e.g., Dugas et al., 2007; see Gentes \& Ruscio, 2011 for a review), and similarly, report a greater range of negative beliefs about the meanings and implications of being uncertain than do non-psychiatric control participants (see Study 1a of this dissertation). In addition, individuals with GAD tend to report negative responses to what non-psychiatric control participants consider "positive uncertainty," such as uncertainty about what one might receive as a gift (Study 1a). People with GAD also engage in an array of behaviours aimed at avoiding and reducing uncertainty (e.g., avoiding activities with uncertain outcomes, checking work over excessively), and do so to the point that these behaviours interfere with their lives (see Study $1 \mathrm{~b}$ of this dissertation). 
Thus, systematically approaching uncertain situations and the discomfort that they elicit should be helpful to people with GAD. Gradually confronting uncertain situations should, theoretically, result in new learning about the degree to which uncertainty is threatening, and foster greater tolerance for uncertainty. This is precisely the aim of a cognitive behavioural therapy (CBT) strategy for GAD that Dugas and Robichaud (2007) call "uncertainty recognition and behavioural exposure” (from here on, uncertainty exposure).

Uncertainty exposure involves psychoeducation about uncertainty, including information about the connection between IU and worry, and the inevitability of uncertainty in daily life. Next a person identifies strategies that he or she uses to avoid or reduce uncertainty (e.g., procrastinating, overpreparing). The person then gradually confronts uncertain situations to purposely induce feelings of uncertainty. This is done either through approaching previously avoided situations with an uncertain outcome (e.g., beginning a vague school project, going on a trip) or refraining from engaging in behaviours aimed at reducing uncertainty (e.g., refraining from seeking reassurance or double-checking). Uncertainty exposure is conducted in an idiographic manner, planned and executed based on the specific behaviours that an individual engages in to avoid or reduce uncertainty (Dugas \& Robichaud, 2007).

The general prediction being tested during uncertainty exposure is the belief that one will not be able to tolerate uncertainty. The uncertainty exposure technique is similar to behavioural experiments in that individuals are asked to record the thoughts they had during and following the uncertain situation, potentially encouraging reappraisal of negative beliefs about uncertainty. In addition, uncertainty exposure is reminiscent of the behavioural experiment strategy because in many cases an individual is not expected to stay in the uncertain situation for a predetermined period of time, or until anxiety decreases, as one would if engaging in traditional exposure. For 
instance, an individual may order an unfamiliar dish at a restaurant and soon after be certain about whether he or she liked it, or a person may stop procrastinating on an ambiguous task and then quickly finish it. In these cases, the person feels uncertain for only a short period of time, and the duration is difficult to predict at the outset of exposure. Thus, uncertainty exposure is constructed more like a behavioural experiment, rather than conventional exposure therapy. Nonetheless, we refer to the strategy as "uncertainty exposure" in the present study, based on its name in Dugas and Robichaud's (2007) treatment manual.

In theory, uncertainty exposure should promote new learning about the degree to which uncertain situations and the internal feeling of being uncertain is threatening, thus it should improve IU. In addition, since IU is strongly associated with a negative attitude toward problems and the problem solving process (i.e., negative problem orientation [NPO]; Dugas et al., 1997, 2007), and by definition, solving problems involves considerable uncertainty; improving one's attitude toward uncertainty should also improve one’s appraisal of problems (Dugas \& Robichaud, 2007). Given that IU and NPO are proposed to underlie and maintain pathological worry (Dugas et al., 1998), targeting IU and NPO should reduce GAD symptoms (Dugas \& Robichaud, 2007).

Although GAD symptoms and IU have been found to improve with a full course of CBT that includes uncertainty exposure as one of its components (CBT-IU; e.g., Dugas et al., 2010; van der Heiden et al., 2012), the effects of uncertainty exposure have not yet been studied in isolation of other treatment strategies. Investigating the effects of uncertainty exposure on IU, NPO, and GAD symptoms is an important step in evaluating whether uncertainty exposure produces the effects it is theorized to have, and in gleaning information about the ways in which it may be further developed. Indeed, given that a substantial proportion ( 30\%) of people with 
GAD neither fully recover nor show high end-state functioning following evidence-based, disorder-specific treatments (e.g., Dugas et al., 2010; Roemer, Orsillo, \& Salters-Pedneault, 2008; Wells et al., 2010), continual efforts to understand the efficacy of specific CBT components and to improve the current GAD treatments are important. Examining the impact of uncertainty exposure is of particular interest as it provides an additional test of Dugas et al.'s IU Theory of GAD by examining whether manipulating a person's approach to uncertainty leads to improvement in IU, NPO, and GAD symptoms. Further, examining the degree to which uncertainty exposure impacts IU, NPO, and GAD symptoms may help to illuminate the mechanisms through which uncertainty exposure has its effects.

Most opportunities to confront uncertainty occur in a person's day-to-day life (Dugas \& Robichaud, 2007). For instance, a person may plan to refrain from seeking reassurance from their spouse when feeling anxious, or engage in an activity with an uncertain outcome, such as a medical exam. Since most uncertainty exposure opportunities occur between therapy sessions, a person’s willingness to complete uncertainty exposure for homework may be particularly important. Research suggests that homework compliance can be an issue in CBT in general (e.g., Antony, Ledley, \& Heimberg, 2005; Leahy, 2005); however, it is not yet understood how homework compliance relates to the use and efficacy of specific CBT strategies, such as uncertainty exposure. Examining the degree to which homework compliance and factors that influence it (e.g., anxiety change expectancy) relate to improvement on GAD outcomes may provide new information about how change occurs with uncertainty exposure. 


\section{Present Study}

A possible first step in testing the efficacy of a specific treatment procedure is to provide a single session of the technique, and then examine its short-term impact on theoretically-relevant symptoms and processes. Studies have demonstrated that a single session of training in exposure or behavioural experiments can produce significant improvement ${ }^{4}$ in anxiety-related symptoms and processes in clinical and analogue anxiety disorder samples (e.g., Fisher \& Wells, 2005; Germain, Shear, Hall, \& Buysse, 2007; Kim, Lundh, \& Harvey, 2002; McManus et al., 2009; McManus, Van Doorn, \& Yiend, 2012; Salkovskis et al., 1999; Rausch, Gramling, \& Auerbach, 2006; Watson, Burley, \& Purdon, 2010). Experiments testing a single session of training provide information about the effects of treatment strategies in a cost- and time-efficient manner before larger-scale investigations of these same strategies are undertaken (Clark-Carter, 2004). Brief intervention studies also provide an efficient means of studying mechanisms of change (e.g., Fisher \& Wells, 2005; Salkovskis, Hackmann, Wells, Gelder, \& Clark, 2007). A single session approach was used in the present study given the lack of research on uncertainty exposure.

The first aim of the present study was to examine whether uncertainty exposure leads to improvement in GAD symptoms, IU, and NPO in people with GAD. The effects of uncertainty exposure on depressive symptoms were also assessed given the high co-occurrence of GAD and depression (e.g., Grant et al., 2005). The effects of a single session of uncertainty exposure training plus 1 week of uncertainty exposure homework prescribed daily (exposure condition) were compared to the effects of assessments only (control condition) on GAD symptoms, depressive symptoms, IU and NPO at baseline, 1-week post baseline, and 2-weeks post baseline. The effects of uncertainty exposure were also assessed using a daily diary wherein participants

\footnotetext{
${ }^{4}$ Effect sizes range from small to large across studies and outcomes assessed.
} 
rated their daily GAD symptoms levels and daily IU cognitions each day for 2-weeks beginning on the first day of the study.

Dugas and colleagues (2007) propose that greater experience with uncertainty promotes learning that uncertainty is not necessarily threatening. It was therefore hypothesized that compared to participants in the control condition, participants in the exposure condition would report improvement from baseline to 1- and 2-weeks following baseline on worry, associated symptoms of GAD (e.g., muscle tension), depressive symptoms, IU and NPO. It was also expected that compared to the control condition, the uncertainty exposure condition would show improvement in ratings of daily outcomes (daily time spent worrying, daily GAD symptom severity, and daily IU cognitions), which were assessed each day for the 2 weeks of this study's duration. However, no specific predictions were made about whether the uncertainty exposure condition would show improved daily outcomes on average across 14 days (i.e., main effect of condition) or over time from day 1 to day 14 (i.e., interaction effects) relative to the control condition, given the limited and mixed findings on how daily worry changes across a full course of CBT (e.g., Donegan \& Dugas, 2012; Ladouceur, Léger, Dugas, \& Freeston, 2004).

The second aim was to investigate the degree to which homework compliance and anxiety change expectancy (i.e., one’s anticipation of being able to change anxiety) relate to improvement following uncertainty exposure. Greater homework compliance has been linked to less severe GAD symptoms following CBT for GAD (Westra, Dozois, \& Marcus, 2007). Anxiety change expectancy is a cognitive factor that has been associated with better homework compliance, and predicts better treatment outcomes (e.g., Price \& Anderson, 2012). Thus, it was hypothesized that in the exposure condition, greater homework compliance and greater anxiety change expectancy would each relate to greater improvement in GAD symptoms, depressive 
symptoms, IU and NPO from baseline to 1- and 2-weeks post baseline, as well as daily time spent worrying, daily GAD symptom severity, and daily IU cognitions. No specific predictions were made about whether daily outcomes would show improvement on average or over time for those with higher levels of homework compliance or anxiety change expectancy, given the lack of research on how daily outcomes are affected by homework and related variables. 


\section{Method}

\section{Participants}

Participants were recruited via flyers posted in the Toronto community and on online forums (e.g., Kijiji). Interested individuals were invited to complete a telephone interview that consisted of the MINI Screen and questions from corresponding modules of the MINI Interview for DSM-IV when questions from the MINI Screen were endorsed. DSM-IV criteria were assessed as the data for the present study were collected before the release of the DSM-5; however, the symptoms of GAD have not changed from DSM-IV to DSM-5 (Starcevic \& Portman, 2013), except for the hierarchical rules (removed in DSM-5) requiring that GAD not be diagnosed when the symptoms occur exclusively during the course of a mood disorder, psychotic disorder, pervasive developmental disorder, or posttraumatic stress disorder. No participants were excluded based on these criteria that were present in DSM-IV, but are not present in DSM5. Individuals were eligible for the present study if they: 1) were 18 to 65 years of age; 2) reported symptoms that met the diagnostic criteria for GAD on the MINI Interview; 3) did not report symptoms of another psychological disorder more severe than GAD on the MINI Interview; 4) did not endorse current mania, psychotic symptoms, substance dependence, or suicidal ideation, intent or plan; 5) were not currently receiving CBT; and 6) were not taking psychotropic medication, or were taking a stable dose of psychotropic medication for at least 2 months. In total, 94 individuals were screened, and 49 met the inclusion criteria. Of individuals who met the inclusion criteria, 40 completed all study components and were included in the analysis. There were 20 individuals in each experimental condition.

Individuals with comorbid diagnoses were included in the present study to increase external validity (Newman et al., 2010). Participants had GAD only (20\%), one comorbid disorder (40\%), two comorbid disorders (38\%), or three or more comorbid disorders (2\%). The 
most common comorbid diagnosis was social anxiety disorder ( $53 \%$ of the sample), followed by major depressive disorder (45\%), obsessive-compulsive disorder (15\%), panic disorder (2.5\%), and specific phobia (2.5\%). There was no significant difference between experimental conditions in the number of comorbid diagnoses (see Table 13).

Although individuals who were currently receiving CBT were excluded, individuals in non-CBT therapy (e.g., mindfulness meditation, supportive therapy, or medication consultations) were included. There were 3 individuals in the exposure condition and 2 in the control condition who were receiving non-CBT therapy; a nonsignificant difference between conditions $\left(\chi^{2}=0.23\right.$, $p=.63$ ). There were also 5 individuals in the exposure condition and 6 in the control condition who were taking a stable dose of psychotropic medication, a nonsignificant difference between conditions $\left(\chi^{2}=0.13, p=.72\right)$.

The present sample was $65 \%$ female, and the mean age was 33 years $(S D=11.04)$. The self-identified ethnicity of participants was as follows: White (70\%), South Asian (10\%), South East Asian (7.5\%), East Asian (5\%), Black (2.5\%), Latin American (2.5\%) and Other (an ethnicity not listed; 2.5\%). There were no significant differences in demographic characteristics between conditions (see Table 13). 
Table 13

Study 2 - Demographic Characteristics by Condition $(N=40)$

\begin{tabular}{|c|c|c|c|}
\hline Characteristic & $\begin{array}{l}\text { Exposure } \\
(n=20)\end{array}$ & $\begin{array}{l}\text { Control } \\
(n=20)\end{array}$ & $t$ or $\chi^{2}$ \\
\hline Age in years $-M(S D)^{\mathrm{a}}$ & $32.21(11.32)$ & $33.84(11.00)$ & 0.45 \\
\hline Sex - Frequency (\%) & & & - \\
\hline Female & $13(65 \%)$ & $13(65 \%)$ & \\
\hline Male & $7(35 \%)$ & $7(35 \%)$ & \\
\hline Race/Ethnicity - Frequency (\%) & & & 6.48 \\
\hline White & $13(65 \%)$ & $15(75 \%)$ & \\
\hline South Asian & $3(15 \%)$ & $1(5 \%)$ & \\
\hline South East Asian & $2(10 \%)$ & $1(5 \%)$ & \\
\hline East Asian & $0(0 \%)$ & $2(10 \%)$ & \\
\hline Latin American & $1(5 \%)$ & $0(0 \%)$ & \\
\hline Black & $0(0 \%)$ & $1(5 \%)$ & \\
\hline Mixed Race & $1(5 \%)$ & $0(0 \%)$ & \\
\hline Marital Status - Frequency (\%) & & & 2.78 \\
\hline Single & $14(70 \%)$ & $9(45 \%)$ & \\
\hline Married/Common-law & $5(25 \%)$ & $8(40 \%)$ & \\
\hline Divorced/Widowed & $1(5 \%)$ & $3(15 \%)$ & \\
\hline Enrolled in Education Program - Frequency (\%) & & & 5.60 \\
\hline University & $7(35 \%)$ & $3(15 \%)$ & \\
\hline College & $0(0 \%)$ & $2(10 \%)$ & \\
\hline Adult Education & $0(0 \%)$ & $2(10 \%)$ & \\
\hline Not Currently Enrolled & $13(65 \%)$ & $13(65 \%)$ & \\
\hline Employment Status - Frequency (\%) & & & 0.43 \\
\hline Not Working (including student) & $8(40 \%)$ & $7(35 \%)$ & \\
\hline Working part-time & $4(20 \%)$ & $3(15 \%)$ & \\
\hline Working full-time & $8(40 \%)$ & $10(50 \%)$ & \\
\hline Number of comorbid diagnoses - $M(S D)$ & $1.47(0.84)$ & $1.05(0.69)$ & 1.73 \\
\hline
\end{tabular}

Note. ${ }^{\mathrm{a}}$ One participant in each condition chose not to report their age. 
In terms of clinical characteristics, all individuals scored above a cut score of 45 that has been used to identify probable GAD on the Penn State Worry Questionnaire (PSWQ; Meyer et al., 1990) (Behar et al., 2003), and 68\% scored above a more stringent cut score of 65 on the PSWQ that is used to distinguish GAD from other anxiety disorders (Fresco et al., 2003). The PSWQ scores of participants with GAD in the present study were comparable to those of individuals with GAD who present for treatment in clinical settings (Dugas et al., 2003, 2010; van der Heiden et al., 2012; Wells et al., 2010).

\section{Measures}

Screening interview. The MINI (Sheehan et al., 1998) is a widely-used semistructured diagnostic interview that assesses for many DSM-IV Axis I disorders. The data for the present study were collected before the release of the DSM-5; however, the core symptoms of GAD have not changed from DSM-IV to DSM-5 (Starcevic \& Portman, 2013). The MINI has very high interrater reliability ( $\kappa s=.88$ to 1.0$)$, good test-retest reliability ( $r s=.76$ to .93 ), and high convergent validity with other clinical interviews (Lecrubier et al., 1997; Sheehan et al., 1998). The MINI Screen (Sheehan et al., 1998) is used in conjunction with the MINI. It consists of 21 closed-ended gateway questions about current symptoms of Axis I disorders, and positive responses on the screen prompt the administration of the corresponding module in the MINI. The MINI Screen has sensitivity ranging from 0.63 to 0.82 , and specificity ranging from 0.61 to 0.83 (Alexander et al., 2008).

\section{General GAD symptom and cognitive process measures.}

The PSWQ (Meyer et al., 1990) is a 16-item self-report "gold standard" measure of the tendency to worry excessively. The PSWQ has high internal consistency ( $\alpha$ s $=.86$ to .91 ; Dear et al., 2011), and a range of test-retest reliabilities ( $r s=.74$ to .92; see Startup \& Erickson, 2006 for a review). 
The PSWQ - Past Week (PSWQ-PW; Stöber \& Bittencourt, 1998) is a 15-item measure of excessive worry over the past week derived from the original PSWQ. The PSWQ-PW excludes one item from the original PSWQ (“I’ve been a worrier all my life”; Stöber \& Bittencourt, 1998). The PSWQ-PW has high internal consistency ( $\alpha$ s $=.84$ to 0.93 ), acceptable test-retest reliability, ( $r s=.32$ to .81 ), and moderate convergent validity with the original PSWQ (Stöber \& Bittencourt, 1998). The PSWQ-PW is sensitive to changes in worry occurring in a short time frame (Stöber \& Bittencourt, 1998).

The Worry and Anxiety Questionnaire (WAQ; Dugas et al., 2001) is an 11-item measure of DSM-IV defined symptoms of GAD. The WAQ contains a 6-item subscale that measures the associated symptoms of GAD (WAQ-A; e.g., fatigue, muscle tension). The WAQ-A has been used in conjunction with the PSWQ to assess the severity of GAD symptoms in prior studies (e.g., Dugas et al., 2010; Dugas et al., 2003). For the present study, the items of the WAQ-A were adapted so that they assessed the severity of GAD-associated symptoms over the past week (from here on, WAQ-A-PW). The WAQ has demonstrated adequate test-retest reliability and good convergent and discriminant validity (Dugas et al., 2001).

The Center for Epidemiological Studies-Depression Scale (CES-D; Radloff, 1977) is a 20-item measure of depressive symptoms experienced over the past week. The CES-D has high internal consistency $(\alpha=.85)$, adequate test-retest reliability $(r=.67)$, and has demonstrated concurrent and construct validity (Radloff, 1977).

The IUS is a 27-item measure of negative beliefs about uncertainty and the consequences of being uncertain. The IUS has demonstrated excellent internal consistency $(\alpha=.95)$, good testretest reliability, $(r=.74)$, and construct validity (Sexton \& Dugas, 2009). 
The Negative Problem Orientation Questionnaire (NPOQ; Robichaud \& Dugas, 2005) is a 12-item measure of the degree to which a person reacts negatively to problems and has negative beliefs about the problem solving process. The NPOQ has good internal consistency ( $\alpha \mathrm{s}=.90$ to .92), good test-retest reliability, $(r=.80)$, and has demonstrated convergent and discriminant validity with other test scores (Robichaud \& Dugas, 2005).

\section{Daily GAD symptom and cognitive process measure.}

The Daily Diary (developed for the present study; see Appendix G) contains 15 items that participants completed once per day for 14 days, starting the day they began the study. One item assessed daily time spent worrying in minutes in an open-ended format (e.g., Verkuil, Brosschot, \& Thayer, 2007), whereas other items were rated from 0 to 10 in terms of severity or agreement with a statement. The diary items were developed based on theory and validated measures of GAD symptoms and cognitive constructs (e.g., WAQ-A, IUS).

With the exception of time spent worrying, which was not grouped with other items because it was unscaled, items assessing the same construct were summed, resulting in two separate composite scores: GAD symptoms and IU cognitions. The daily GAD symptoms score comprised responses to seven items that assessed the severity of worry and GAD-associated symptoms (e.g., restlessness). The daily IU cognitions score comprised responses to seven items assessing the severity of negative beliefs about uncertainty and its implications. Items assessing daily IU cognitions were either based on the underlying factors of the IUS (e.g., "I felt that uncertainty was unfair today”; Sexton \& Dugas, 2009), or were designed to tap beliefs in one’s ability to cope with uncertainty (e.g., "I felt able to cope with feeling uncertain today”). The latter items were included based on theory suggesting that one's perceived ability to cope with uncertainty should improve with uncertainty exposure (Dugas \& Robichaud, 2007). 
Internal consistencies for the two composite scores were calculated at baseline, day 7 (i.e., 1 week post baseline), and day 14 (i.e., 2 weeks post baseline), and were high for GAD symptoms ( $\alpha \mathrm{s}=0.80$ to 0.92 ) and adequate for IU cognitions ( $\alpha \mathrm{s}=0.72$ to 0.94 ). Validity was assessed by calculating the association of the composite scores to scores on similar validated measures at baseline, day 7, and day 14 . Daily GAD symptoms were significantly associated with the PSWQ-PW and WAQ-A at each time point ( $r s=.40$ to $.67 ; p s<.03)$. Daily IU cognitions were not associated with the IUS at baseline $(r=.17, p=.31)$, but were significantly associated with the IUS at day 7 and day $14(r s=.34$ and $.49, p s<.03)$.

\section{Homework compliance, anxiety change expectancy, and exposure monitoring.}

The Homework Compliance Scale (HCS; adapted from Primakoff, Epstein, \& Covi, 1986) measures the degree to which respondents engaged in uncertainty exposure homework each day for 7 days. Each day participants provided one of the following homework ratings: $1=\mathrm{I}$ did not attempt an uncertainty exposure; 2 = I attempted to plan an uncertainty exposure, but was unable to execute it; 3 = I did something related to uncertainty exposure, but it was different than an uncertainty exposure; 4 = I did some portion of an uncertainty exposure; 5 = I completed an uncertainty exposure; and 6 = I did more than one uncertainty exposure. Ratings from each day were summed to produce a total HCS score.

The Anxiety Change Expectancy Scale (ACES; Dozois \& Westra, 2005) is a 20-item measure of general beliefs about the degree to which one's anxiety can improve. The ACES has excellent internal consistency $(\alpha=.92)$ and good convergent and divergent validity (Dozois \& Westra, 2005).

The Uncertainty Exposure Practice Form (Dugas \& Robichaud, 2007) is a handout from Dugas and Robichaud's (2007) manual for the cognitive behavioural treatment of GAD. Clients 
use the form to record descriptions of each uncertainty exposure, their thoughts during and after the exposure, and their discomfort during the exposure, from 0 (none at all) to 100 (extreme).

\section{Procedure}

Eligibility was assessed over the telephone using the MINI Screen, modules of the MINI Interview, and questions that assessed the other eligibility criteria (see Participants). Eligible participants were scheduled for three visits to the laboratory that were spaced approximately 1 week apart. Informed consent was obtained prior to all study procedures (see Appendix D).

Visit 1 - part A. Each participant completed the PSWQ-PW, WAQ-A-PW, CES-D, IUS, NPOQ, and ACES. The PSWQ was also administered to characterize the sample and establish that both conditions were equivalent on the general tendency to worry. The experimenter then explained how to complete the 14-day Daily Diary. Participants were randomly assigned to either the exposure condition or control condition. Those who were assigned to the control condition completed Visit 1 - part A only (i.e., assessment only), and individuals assigned to the exposure condition went on to complete Visit 1 - part B. Participants assigned to the control condition were told that they would have the chance to receive the exposure training after completing the final assessment.

Visit 1 - part B. Participants received 1 hour of training in uncertainty exposure based on Dugas and Robichaud's (2007) protocol, including: psychoeducation about IU and worry, identifying behaviours that the participant was using to avoid and minimize uncertainty in their daily life, and discussing the rationale for uncertainty exposure. Next, seven uncertainty exposures were planned, one for each day of the coming week, and participants were asked to complete one uncertainty exposure each day. Exposures were planned by orienting participants to common strategies that individuals may use to eliminate or avoid uncertainty (e.g., seeking reassurance from others, avoiding activities with an uncertain outcome), which were drawn from 
Dugas and Robichaud's (2007) CBT-IU treatment manual. Then participants identified the strategies they used most often to eliminate or avoid uncertainty, and each person's exposures were based on refraining from engaging in these personally relevant strategies. During exposure planning, the experimenter encouraged individuals to engage in a variety of different "types" of exposure (e.g., approach a previously avoided uncertain situation, reduce time spent overpreparing). Participants were given instructions on completing the Uncertainty Exposure Practice Form and were asked to complete one form per exposure. Participants were also given instructions on how to complete the HCS, and were asked to complete it daily over the coming week.

Visits 2. One week following Visit 1, all participants returned to the lab and completed the questionnaires that they completed during Visit 1 - part A. They also were reminded to continue to complete the Daily Diary for another 7 days. Participants in the exposure condition returned the Uncertainty Exposure Practice Forms and the HCS.

Visit 3. Two weeks following Visit 1, all participants completed questionnaires that they completed during Visit 1 - part A and Visit 2, and returned the 14-day Daily Diary. After all study components were completed participants were debriefed (see Appendix F) and compensated.

At the time of informed consent, participants were told that if they were assigned to the control condition they could receive exposure training following their participation in the experiment. With only a few exceptions, participants assigned to the control condition expressed an interest in and received exposure training following the experiment. 


\section{Results}

\section{Preliminary Analyses}

Impact of current medication or psychotherapy on outcomes. The impact of including individuals taking a stable dose of psychotropic medication or currently receiving nonCBT psychotherapy on study outcomes was assessed. Point-biserial correlations were calculated given that currently taking medication and currently receiving non-CBT psychotherapy were binary variables. The results showed no significant correlations between current medication or current psychotherapy and scores on the PSWQ-PW, WAQ-A-PW, CES-D, IUS, or NPOQ at 1or 2-week post baseline ( $r s=-.17$ to $.30, p s>.06)$.

Descriptive information on uncertainty exposure. Based on responses on the HCS, 72\% of participants completed 5 or more exposures for homework (ratings of 5 or 6 on the HCS for at least 5 of 7 days), and 55\% completed 6 or more exposures (ratings of 5 or 6 on the HCS for at least 6 of 7 days). The uncertainty exposures that participants described engaging in were coded into themes and are displayed in Table 14. On average, people completed 3.55 (SD = 1.10) different "types” of exposure (e.g., refrained from excessive checking, refrained from overpreparing). The percentage of individuals completing each type of exposure, and examples of each type of exposure are shown in Table 14. Participants' discomfort ratings during exposure ranged from 17 to 75 on a scale of 0 (none at all) to 100 (extreme), with an average of $55.61(S D=14.52)$ across all exposures recorded. Participants' discomfort during exposure was not significantly associated with change on any GAD outcome from baseline to 1- or 2-weeks post baseline ( $r \mathrm{~s}=-.31$ to $.17, \mathrm{ps}>.05)$. 
Table 14

Types of Uncertainty Exposures that Participants with GAD Completed $(n=20)$

\begin{tabular}{|c|c|c|}
\hline Туре & $\begin{array}{l}\text { \% } \\
\text { Exposure } \\
\text { Group }\end{array}$ & Examples \\
\hline $\begin{array}{l}\text { Approached a } \\
\text { previously avoided } \\
\text { situation with an } \\
\text { unknown outcome }\end{array}$ & $76 \%$ & $\begin{array}{l}\text { - Read about a health problem that I worry about for } 20 \\
\text { minutes } \\
\text { - Tried a new exercise at the gym } \\
\text { - Spent an hour organizing financial papers } \\
\text { - Called a college department and asked questions about a } \\
\text { program }\end{array}$ \\
\hline $\begin{array}{l}\text { Approached a } \\
\text { previously } \\
\text { procrastinated } \\
\text { uncertain task }\end{array}$ & $43 \%$ & $\begin{array}{l}\text { - Applied to } 3 \text { jobs } \\
\text { - Started writing a paper for } 2 \text { hours } \\
\text { - Went to get blood work done }\end{array}$ \\
\hline $\begin{array}{l}\text { Refrained } \\
\text { from/reduced } \\
\text { reassurance } \\
\text { seeking }\end{array}$ & $62 \%$ & $\begin{array}{l}\text { - Asked my partner how my cooking was } 1 \mathrm{x} \text { only } \\
\text { - Refrained from seeking reassurance from classmates after a } \\
\text { presentation } \\
\text { - Delayed asking for reassurance from my son about selling } \\
\text { my house for } 1 \text { day }\end{array}$ \\
\hline $\begin{array}{l}\text { Refrained } \\
\text { from/reduced } \\
\text { excessive checking }\end{array}$ & $57 \%$ & $\begin{array}{l}\text { - Reduced checking voicemail from } 15 \text { to } 10 \text { times on } \\
\text { Monday } \\
\text { - Checked over request } 2 \mathrm{x} \text { only before submitting } \\
\text { - Proofread assignment } 1-2 \mathrm{x} \text { only }\end{array}$ \\
\hline $\begin{array}{l}\text { Refrained } \\
\text { from/reduced } \\
\text { over-preparing }\end{array}$ & $29 \%$ & $\begin{array}{l}\text { - Left the house without redoing my hair } \\
\text { - Reduced time (from } 1 \text { hour to } 30 \text { mins) spent planning out } \\
\text { my week } \\
\text { - Did not rehearse what I would say on a phone call to a } \\
\text { friend }\end{array}$ \\
\hline $\begin{array}{l}\text { Refrained } \\
\text { from/reduced } \\
\text { attempts to control } \\
\text { a situation }\end{array}$ & $43 \%$ & $\begin{array}{l}\text { - Did not schedule plans for Saturday } \\
\text { - Asked for and accepted help from my roommate with } \\
\text { cooking } \\
\text { - Refrained from correcting my colleague's work 5x during } \\
\text { the day }\end{array}$ \\
\hline $\begin{array}{l}\text { Refrained } \\
\text { from/reduced } \\
\text { excessive } \\
\text { information } \\
\text { seeking }\end{array}$ & $14 \%$ & $\begin{array}{l}\text { - Watched a movie without looking up reviews first } \\
\text { - Spent only } 10 \text { minutes comparing restaurant menus/food } \\
\text { information } \\
\text { - Spent less time looking up transit information before an } \\
\text { outing }\end{array}$ \\
\hline
\end{tabular}


Note. Percentage of participants who engaged in each exposure type do not add to $100 \%$ as most individuals engaged in more than one exposure type over 1 week $(M=3.55, S D=1.10)$. Data were gathered through a form that participants completed called the Uncertainty Exposure Practice Form. 


\section{Effects of Uncertainty Exposure on Weekly or Dispositional Outcome Measures}

It was of interest to identify the impact of uncertainty exposure on GAD symptoms, depressive symptoms, IU and NPO. It was hypothesized that participants in the exposure condition would show greater improvement in these outcomes than would participants in the control condition. Means and standard deviations are displayed in Table 15. Mixed 2 (Condition: exposure, control) by 3 (Time: baseline, 1-week post baseline, 2-week post baseline) Analyses of Variance (ANOVAs) were conducted on each outcome measure, and then interaction effects were examined using Bonferroni pairwise tests. As the objectives were to investigate differences between the exposure and control conditions over time, only pairwise tests of the interaction effects are reported. Given the present study's hypotheses, even when omnibus interaction effects were nonsignificant, they were followed up with pairwise contrasts (see Hancock \& Klockars, 1996 for a detailed rationale). There were no significant betweencondition differences on any variables at baseline.

\section{Effects of uncertainty exposure on GAD symptoms and depression.}

PSWQ-PW. A mixed ANOVA showed a significant main effect of Time, $F(2,37)=$ 4.35, $p=.02, \eta_{\mathrm{p}}{ }^{2}=.19$, no significant main effect of Condition, $F(1,38)=2.26, p=.14, \eta_{\mathrm{p}}^{2}=.06$, $d_{\mathrm{bs}}=0.39$, and a significant interaction effect, $F(2,37)=3.73, p=.03, \eta_{\mathrm{p}}{ }^{2}=.17$. Pairwise tests of the interaction effect showed that in the exposure condition, PSWQ-PW scores were significantly lower at 1 - and 2-weeks post baseline compared to at baseline $\left(p<.01, d_{\mathrm{ws}}=0.65\right.$, and $\left.p=.02, d_{\mathrm{ws}}=0.55\right)$; with no significant difference between the post baseline scores $(p=$ $\left.1.00, d_{\mathrm{ws}}=0.06\right)$. 
Table 15

Scores on GAD-Related Symptoms and Cognitions Separated by Condition $(N=40)$

\begin{tabular}{|c|c|c|c|c|}
\hline Measure & Condition & $\begin{array}{l}\text { Baseline } \\
\text { M (SD) }\end{array}$ & $\begin{array}{c}\text { 1-Week Post } \\
\text { baseline } \\
\text { M (SD) }\end{array}$ & $\begin{array}{c}\text { 2-Week Post } \\
\text { baseline } \\
\text { M (SD) }\end{array}$ \\
\hline \multirow[t]{2}{*}{ PSWQ-PW } & Exposure & $67.05(12.81)$ & $57.80(12.57)$ & $57.35(13.72)$ \\
\hline & Control & 66.97 (10.87) & 67.45 (9.92) & $62.45(15.08)$ \\
\hline \multirow[t]{2}{*}{ WAQ-A-PW } & Exposure & $20.00(4.01)$ & $17.85(5.03)$ & 18.05 (5.96) \\
\hline & Control & $22.55(4.53)$ & $22.60(4.07)$ & 21.30 (5.99) \\
\hline \multirow[t]{2}{*}{ CES-D } & Exposure & 31.15 (14.08) & $24.65(12.21)$ & $24.70(13.10)$ \\
\hline & Control & $27.53(8.64)$ & $27.90(10.03)$ & $24.83(9.52)$ \\
\hline \multirow[t]{2}{*}{ IUS } & Exposure & $101.95(15.42)$ & $96.30(15.61)$ & $91.20(18.17)$ \\
\hline & Control & $97.53(19.22)$ & 99.25 (15.68) & $97.50(17.49)$ \\
\hline \multirow[t]{2}{*}{ NPOQ } & Exposure & $46.10(9.34)$ & 42.55 (9.55) & 38.75 (10.93) \\
\hline & Control & $40.23(12.29)$ & 41.75 (11.06) & $41.00(11.21)$ \\
\hline
\end{tabular}

Note. PSWQ-PW = Penn State Worry Questionnaire - Past Week; WAQ-A-PW = Worry and Anxiety Questionnaire - Associated Symptoms Subscale - Past Week; CES-D = Center for Epidemiological Studies Depression Scale; IUS = Intolerance of Uncertainty Scale; NPOQ = Negative Problem Orientation Questionnaire. There were no significant differences between conditions on any outcome measure at baseline. 
In the control condition, PSWQ-PW scores were not significantly different from baseline to 1- or 2-weeks post baseline ( $p=1.00, d_{\mathrm{ws}}=-0.04$, and $p=.58, d_{\mathrm{ws}}=.38$ ); however, the control condition showed a significant reduction in PSWQ-PW scores between 1- and 2-weeks post baseline ( $p=.05, d_{\mathrm{ws}}=.57$ ). At 1-week post baseline, participants in the exposure condition showed significantly lower PSWQ-PW scores than did participants in the control condition ( $p=$ $.01, d_{\mathrm{bs}}=.85$ ), although there was no significant difference between conditions at 2-weeks post baseline $\left(p=.27, d_{\mathrm{bs}}=0.35\right)$.

WAQ-A-PW. A mixed ANOVA showed no significant main effect of Time, $F(2,37)=$ 2.99, $p=.06, \eta_{\mathrm{p}}^{2}=.14$, but a significant main effect of Condition, $F(1,38)=6.48, p=.02, \eta_{\mathrm{p}}^{2}=$ $.15, d_{\mathrm{bs}}=0.71$. There was no significant interaction effect, $F(2,37)=2.18, p=.13, \eta_{\mathrm{p}}^{2}=.11$. Pairwise tests of the interaction effect showed that in the exposure condition, WAQ-A-PW scores were significantly lower at 1 -week post baseline compared to baseline $\left(p=.02, d_{\mathrm{ws}}=\right.$ $0.54)$, but this effect was no longer significant at 2-weeks post baseline ( $p=.21, d_{\mathrm{ws}}=0.39$ ). There was no significant difference between post baseline scores $\left(p=1.00, d_{\mathrm{ws}}=-0.05\right)$. In the control condition, WAQ-A-PW scores were not significantly different from baseline to 1- or 2weeks post baseline ( $p=1.00, d_{\mathrm{ws}}=-0.02$, and $p=.71, d_{\mathrm{ws}}=0.33$ ); with no significant difference between the post baseline scores ( $p=.61, d_{\mathrm{ws}}=0.32$ ). At 1 -week post baseline, participants in the exposure condition showed significantly lower WAQ-A-PW scores than participants in the control condition ( $\left.p<.01, d_{\mathrm{bs}}=1.04\right)$, although the difference was not significant at 2-weeks post baseline $\left(p=.09, d_{\mathrm{bs}}=0.54\right)$.

CES-D. A mixed ANOVA showed a significant main effect of Time, $F(2,37)=3.72, p$ $=.03, \eta_{\mathrm{p}}^{2}=.17$, no main effect of Condition, $F(1,38)=0.01, p=.98, \eta_{\mathrm{p}}^{2}<0.01, d_{\mathrm{bs}}=0.01$, and no significant interaction effect, $F(2,37)=2.95, p=.07, \eta_{\mathrm{p}}{ }^{2}=.14$. Pairwise tests of the 
interaction effect showed that in the exposure condition, CES-D scores were significantly lower at 1 - and 2-weeks post baseline compared to baseline $\left(p<.01, d_{\mathrm{ws}}=0.79\right.$, and $p=.03, d_{\mathrm{ws}}=$ 0.57), with no significant difference between the post baseline scores $\left(p=1.00, d_{\mathrm{ws}}<-0.01\right)$. In the control condition, CES-D scores were not significantly different from baseline to 1- or 2weeks post baseline ( $p=1.00, d_{\mathrm{ws}}=-0.04$, and $p=.81, d_{\mathrm{ws}}=0.27$ ), with no significant difference between post baseline scores $\left(p=.41, d_{\mathrm{ws}}=0.36\right)$. There were no significant between-condition differences in CES-D scores 1 - or 2-weeks post baseline $\left(p=.36, d_{\mathrm{bs}}=0.29\right.$, and $p=.97$, $d_{\mathrm{bs}}=$ $0.01)$.

\section{Effects of uncertainty exposure on IU and NPO.}

IUS. A mixed ANOVA showed no significant main effect of Time, $F(2,37)=1.89, p=$ $.17, \eta_{\mathrm{p}}{ }^{2}=.09$, or Condition, $F(1,38)=0.12, p=.73, \eta_{\mathrm{p}}^{2}<0.01, d_{\mathrm{bs}}=0.10$, and no significant interaction effect, $F(2,37)=2.00, p=.15, \eta_{\mathrm{p}}^{2}=.10$. Pairwise tests of the interaction effect showed that in the exposure condition, IUS scores were not significantly different from baseline at 1 -week post baseline ( $\left.p=.12, d_{\mathrm{ws}}=0.49\right)$, but were significantly lower than baseline at 2weeks post baseline ( $\left.p=.04, d_{\mathrm{ws}}=0.56\right)$; with no significant difference between the post baseline scores $\left(p=.19, d_{\mathrm{ws}}=0.41\right)$. In the control condition, IUS scores were not significantly different from baseline to 1 - or 2-weeks post baseline ( $p=1.00, d_{\mathrm{ws}}=-0.14$, and $\left.p=1.00, d_{\mathrm{ws}}<0.01\right)$; with no significant difference between the post baseline scores $\left(p=1.00, d_{\mathrm{ws}}=0.16\right)$. The IUS scores of the exposure condition were not significantly different than those of the control condition at 1 - or 2 -weeks post baseline $\left(p=.55\right.$, $d_{\mathrm{bs}}=0.19$, and $\left.p=.27, d_{\mathrm{bs}}=0.35\right)$. 
NPOQ. A mixed ANOVA showed no significant main effect of Time, $F(2,37)=2.32, p$ $=.11, \eta_{\mathrm{p}}^{2}=.11$, or Condition, $F(1,38)=0.24, p=.63, \eta_{\mathrm{p}}^{2}<0.01, d_{\mathrm{bs}}=0.14$, but a significant interaction effect, $F(2,37)=3.38, p=.05, \eta_{\mathrm{p}}^{2}=.15$. Pairwise tests of the interaction effect showed that in the exposure condition, NPOQ scores did not differ significantly from baseline to 1-week post baseline ( $p=.13, d_{\mathrm{ws}}=0.51$ ), but were significantly lower 2-weeks post baseline compared to baseline ( $p<.01, d_{\mathrm{ws}}=0.82$ ), with no significant difference between the post baseline scores $\left(p=.10, d_{\mathrm{ws}}=0.51\right)$. In the control condition, NPOQ scores were not significantly different from baseline to 1 - or 2-weeks post baseline ( $p=1.00, d_{\mathrm{ws}}=-0.19$, and $p$ $\left.=1.00, d_{\mathrm{ws}}=-0.07\right)$; with no significant difference between the post baseline scores ( $p=1.00, d_{\mathrm{ws}}$ $=0.10$ ). There were no significant between-condition differences in NPOQ scores at 1-or 2weeks post baseline $\left(p=.81, d_{\mathrm{bs}}=0.08\right.$, and $p=.52$, $\left.d_{\mathrm{bs}}=0.20\right)$.

\section{Exploratory Posthoc Analysis of the Relation of GAD symptoms to IU and NPO}

Three steps were taken to assess the degree to which improvements in weekly GAD symptoms (PSWQ-PW and WAQ-A-PW) were associated with improvements in IUS and NPOQ scores based on a method used previously to assess the degree to which change in symptoms was accounted for by change in cognitions (Koerner, Antony, Young, \& McCabe, 2013). First, the standardized residualized change scores were calculated for the IUS and NPOQ from baseline to 1- and 2-weeks post baseline from the raw change scores for the IUS and NPOQ (see Steketee \& Chambless, 1992). Second, zero-order correlations of the standardized residualized change scores on the IUS and NPOQ to scores on the PSWQ-PW and WAQ-A-PW at 1- and 2-weeks post baseline were calculated in the exposure and control conditions separately (see Table 16). 
Table 16

Summary of Zero-Order Correlations between Change in IU-Related Cognitions and GAD Symptoms in the Exposure and Control Conditions $(N=40)$

\begin{tabular}{|c|c|c|c|}
\hline \multirow{2}{*}{ Condition } & \multirow{2}{*}{$\begin{array}{c}\text { GAD Symptom } \\
\text { Variables }\end{array}$} & \multicolumn{2}{|c|}{ Change in IU-Related Cognitions } \\
\hline & & $\begin{array}{c}\Delta \text { IUS } \\
\text { week } 1\end{array}$ & $\begin{array}{c}\triangle \text { NPOQ } \\
\text { week } 1\end{array}$ \\
\hline \multirow[t]{2}{*}{ Exposure } & PSWQ-PW-week 1 & $.50 *$ & .42 \\
\hline & WAQ-A-PW-week 1 & $.62 * *$ & .36 \\
\hline \multirow[t]{4}{*}{ Control } & PSWQ-PW-week 1 & .34 & $.48 *$ \\
\hline & WAQ-A-PW-week 1 & .14 & .37 \\
\hline & & $\Delta$ IUS & $\triangle \mathrm{NPOQ}$ \\
\hline & & week 2 & week 2 \\
\hline \multirow[t]{2}{*}{ Exposure } & PSWQ-PW-week 2 & $.58 * *$ & $.45^{*}$ \\
\hline & WAQ-A-PW-week 2 & $.59 * *$ & .39 \\
\hline \multirow[t]{2}{*}{ Control } & PSWQ-PW-week 2 & .21 & .27 \\
\hline & WAQ-A-PW-week 2 & .07 & .32 \\
\hline \multicolumn{4}{|c|}{$\begin{array}{l}\text { Notes. PSWQ-PW = Penn State Worry Questionnaire - Past Week; WAQ-A-PW = Worry and } \\
\text { Anxiety Questionnaire - Associated Symptoms Subscale - Past Week; IUS = Intolerance of } \\
\text { Uncertainty Scale; NPOQ = Negative Problem Orientation Questionnaire. Change scores on } \\
\text { outcome variables were calculated by subtracting scores on a measure at 1-week post baseline or } \\
\text { 2- weeks post baseline from scores on the same measure at baseline; and then converting the raw } \\
\text { scores to standardized residualized change scores. The top panel shows correlations at week 1, } \\
\text { and the bottom panel shows correlations at week 2. Two-tailed correlations. }\end{array}$} \\
\hline \multicolumn{2}{|c|}{$* p<.05, * * p<.01$} & & \\
\hline
\end{tabular}


Third, all significant zero-order correlations between change in IU or NPO and GAD symptoms at 1- or 2-weeks post baseline were earmarked for further examination using multiple regression analyses (Koerner et al., 2013). To examine the unique contribution of improvement on IU or NPO to variance in GAD symptoms at the post baseline assessments, multiple regression analyses were conducted separately for each time period (baseline to 1-week post baseline; baseline to 2-weeks post baseline) within each condition (exposure and control). Two or fewer cases per model were found to have undue influence on results (i.e., Cook's distance $>1$ ) and were excluded (Field, 2009). Indicators of multicollinearity were within acceptable limits (Field, 2009), and all other assumptions of multiple regression were satisfied.

Correlations in the exposure condition showed that the IUS change scores from baseline to 1- and 2-weeks post baseline were significantly related to PSWQ-PW and WAQ-A-PW scores at both time points (see Table 16). In addition, NPOQ change scores from baseline to 2-weeks post baseline were significantly related to PSWQ-PW scores at 2-weeks post baseline. No residualized change scores were significantly associated with GAD symptoms in the control condition, with one exception; NPOQ change scores from baseline to 1-week post baseline were significantly related to PSWQ-PW scores at 1-week post baseline.

Multiple regression analyses (see Table 17) were subsequently conducted in the exposure and control conditions using the following outcomes: PSWQ-PW at 1-week post baseline, WAQ-A-PW at 1-week post baseline, PSWQ-PW at 2-weeks post baseline, and WAQ-A-PW at 2-weeks post baseline. The baseline scores on the outcome measure in each analysis were entered into step 1 of each regression. Then the IU-related cognitions that were significantly associated with the outcome measure in each analysis (see Table 16) were entered as predictor variables into step 2 of each regression. 
Table 17

Multiple Regressions Predicting Weekly GAD Symptoms at 1- and 2-Week Post Baselines from Improvement on IU-Related Cognitions

\begin{tabular}{|c|c|c|c|c|c|c|c|}
\hline Condition & $\begin{array}{l}\text { Outcome } \\
\text { Variable }\end{array}$ & Predictor Variable & $\Delta R^{2}$ & $\Delta F$ & $B$ & $S E$ & $\beta$ \\
\hline Exposure & $\begin{array}{l}\text { PSWQ-PW } \\
\text { week } 1\end{array}$ & $\begin{array}{c}\text { PSWQ-PW baseline } \\
\Delta \text { IUS- week } 1\end{array}$ & 0.39 & $14.53 * *$ & $\begin{array}{c}.77 \\
7.08\end{array}$ & $\begin{array}{l}.26 \\
1.86\end{array}$ & $\begin{array}{l}.49 * * \\
.62 * *\end{array}$ \\
\hline Exposure & $\begin{array}{l}\text { WAQ-A-PW } \\
\text { week } 1\end{array}$ & $\begin{array}{c}\text { WAQ-A-PW baseline } \\
\Delta \text { IUS- week } 1\end{array}$ & 0.39 & $26.58 * *$ & $\begin{array}{c}.79 \\
3.14\end{array}$ & $\begin{array}{l}.15 \\
.61\end{array}$ & $\begin{array}{l}.63 * * \\
.62 * *\end{array}$ \\
\hline Control & $\begin{array}{l}\text { PSWQ-PW } \\
\text { week } 1\end{array}$ & $\begin{array}{c}\text { PSWQ-PW baseline } \\
\Delta \text { NPOQ- week } 1\end{array}$ & 0.34 & $13.47 * *$ & $\begin{array}{c}.53 \\
5.33 \\
\end{array}$ & $\begin{array}{c}.17 \\
1.45 \\
\end{array}$ & $\begin{array}{l}.49 * * \\
.58 * * \\
\end{array}$ \\
\hline Exposure & $\begin{array}{l}\text { PSWQ-PW } \\
\text { week } 2\end{array}$ & $\begin{array}{c}\text { PSWQ-PW baseline } \\
\Delta \text { IUS- week } 2 \\
\Delta \text { NPOQ- week } 2\end{array}$ & 0.67 & $19.55 * *$ & $\begin{array}{l}.37 \\
2.52 \\
6.76\end{array}$ & $\begin{array}{c}.20 \\
2.79 \\
3.09\end{array}$ & $\begin{array}{l}.26 \\
.25 \\
.60 *\end{array}$ \\
\hline Exposure & $\begin{array}{l}\text { WAQ-A-PW } \\
\text { week } 2\end{array}$ & $\begin{array}{c}\text { WAQ-A-PW baseline } \\
\Delta \text { IUS- week } 2\end{array}$ & 0.25 & $18.49 * *$ & $\begin{array}{l}1.14 \\
2.70\end{array}$ & $\begin{array}{l}.18 \\
.63\end{array}$ & $\begin{array}{l}.74 * * \\
.50 * *\end{array}$ \\
\hline \multicolumn{8}{|c|}{$\begin{array}{l}\text { Note. PSWQ-PW = Penn State Worry Questionnaire - Past Week; WAQ-A-PW = Worry and } \\
\text { Anxiety Questionnaire - Associated Symptoms Subscale - Past Week; IUS = Intolerance of } \\
\text { Uncertainty Scale; NPOQ = Negative Problem Orientation Questionnaire. Change scores on } \\
\text { outcome variables were calculated by subtracting scores on a measure at 1-week post baseline or } \\
\text { 2- weeks post baseline from scores on the same measure at baseline; and then converting the raw } \\
\text { scores to standardized residualized change scores. Results shown are the final step of the } \\
\text { regression analyses. }\end{array}$} \\
\hline
\end{tabular}


In the exposure condition, change in IUS scores from baseline to 1-week post baseline made a significant unique contribution to PSWQ-PW scores at 1-week post baseline. In addition, change in NPOQ scores from baseline to 2-weeks post baseline made a significant unique contribution to PSWQ-PW scores at 2-weeks post baseline. Change in IUS scores from baseline to 1- and 2-weeks post baseline made a significant unique contribution to WAQ-A-PW scores at 1- and 2-weeks post baseline, respectively.

In the control condition, change in NPOQ scores from baseline to 1-week post baseline made a significant unique contribution to PSWQ-PW scores at 1-week post baseline; however there were no other associations between IU-related cognitions and GAD symptoms in the control condition.

\section{Effects of Uncertainty Exposure on Daily Outcomes}

Another study objective was to examine the impact of uncertainty exposure on daily GAD symptoms and daily IU-related cognitions. Daily assessments over 2 weeks (time; level 1 predictor) were nested within participants assigned to the exposure or control condition (condition; level 2 predictor). Hierarchical linear modeling (HLM; Raudenbush, Bryk, Cheong, Congdon, \& du Toit, 2004) was used to examine between-condition differences in daily outcomes on average (i.e., main effect of condition) and the rate of change on daily outcomes over time (i.e., time x condition interactions). It was expected that the exposure condition would show greater improvement in daily outcomes compared to the control condition.

Three analyses were conducted: on daily worry time, daily GAD symptoms, and daily IU cognitions, respectively. The assumptions of HLM were met with one exception; the daily worry time data were right-skewed. Research has shown HLM to be robust to violations of the assumption of normality (e.g., Maas \& Hox, 2004), so the daily worry time data were not transformed. The interclass correlation coefficients (ICCs) in each of the three models were 
significant (ICCs $=0.51$ to $0.53, p s<.01$ ), confirming the intercorrelated nature of the daily data, and the necessity of a hierarchical data analytic approach. In each of the three analyses, the covariance structure that was the most appropriate fit for the data was the random intercept and random slope uncorrelated model.

The results of the HLM analyses are displayed in Table 18. There were significant main effects of condition on daily worry time and daily GAD symptom severity. Specifically, collapsed across time, individuals in the exposure condition spent less time worrying $(M=133$ min per day, $S D=19.29$ mins $)$ than did individuals in the control condition $(M=170.36$ min per day, $S D=19.90 \mathrm{~min}$ ) during the 2 weeks that followed the initial assessment. Similarly, individuals in the exposure condition endorsed less severe GAD symptoms $(M=4.38, S D=$ 2.18) during the 2-week monitoring period than did individuals in the control condition $(M=$ $5.46, S D=2.20)$. There was no main effect of condition on daily IU cognitions. The results also showed no significant time x condition interaction effects on any of the outcome variables, suggesting that the rate of change on daily outcomes over 2 weeks did not differ between conditions. 
Table 18

Multilevel Models for Daily Outcomes as Associated with Time and Condition $(N=40)$

\begin{tabular}{|c|c|c|c|c|}
\hline Outcome & Fixed effects & $b$ & $S E$ & $t$ \\
\hline Daily Worry & Intercept & 129.58 & 19.59 & $6.62 * *$ \\
\hline \multirow[t]{3}{*}{ Time } & Time & 0.45 & 1.86 & 0.24 \\
\hline & Condition & 62.85 & 27.70 & $2.27 *$ \\
\hline & Time $\mathrm{x}$ Condition & -3.28 & 2.67 & -1.23 \\
\hline Daily GAD & Intercept & 4.67 & 0.37 & $12.62 * *$ \\
\hline \multirow[t]{3}{*}{ Symptoms } & Time & -0.04 & 0.03 & -1.21 \\
\hline & Condition & 1.09 & 0.52 & $2.09 *$ \\
\hline & Time x Condition & -0.001 & 0.04 & -0.02 \\
\hline Daily IU & Intercept & 4.34 & 0.39 & $11.26 * *$ \\
\hline \multirow[t]{3}{*}{ Cognitions } & Time & -0.04 & 0.03 & -1.43 \\
\hline & Condition & 0.31 & 0.54 & 0.57 \\
\hline & Time x Condition & 0.03 & 0.04 & 0.75 \\
\hline
\end{tabular}

Note. ${ }^{*} p<.05 ; * * p<.01$. 


\section{Association of Homework and Anxiety Change Expectancy to Outcomes}

Equivalence of anxiety change expectancy at baseline. The exposure and control

group's expectations for change as measured by the ACES were examined to assess the degree to which expectations differed between groups. The exposure and control conditions had comparable anxiety change expectancies at baseline $(M=75.19, S D=12.88, M=74.50, S D=$ 11.88, respectively; $t(38)=-.018, p=.86), 1$ week post baseline $(M=72.24, S D=20.60, M=$ 74.85, $S D=10.32$, respectively; $t(38)=0.51, p=.62)$, and 2 weeks post baseline $(M=77.50, S D$ $=12.42, M=78.26, S D=12.65$, respectively; $t(38)=0.19, p=.85)$. The mean score on the ACES in the present sample was $74.85(S D=12.24)$ on a scale of 20 and 100 , which is above the midpoint score of the scale (60) but below the top quartile of the scale (80 and above).

Weekly and dispositional outcomes. It was hypothesized that higher scores the HCS and ACES would be associated with greater improvement on weekly and dispositional outcomes from baseline to 1- and 2-weeks post baseline in those who received uncertainty exposure training. Pearson correlations showed that higher HCS scores and higher ACES scores were each significantly associated with greater improvement on PSWQ-PW and WAQ-A-PW scores at 1-week post-baseline. Higher HCS scores were significantly associated with greater improvement on PSWQ-PW scores at 2-weeks post baseline, and higher ACES scores were significantly correlated with greater improvement on PSWQ-PW and IUS scores at 2-weeks post baseline (see Tables 19 and 20). 
Table 19

Zero-Order Correlations of Homework Compliance and Anxiety Change Expectancy to Change Scores on GAD-Related Outcomes from Baseline to 1-Week Post Baseline in the Exposure Condition $(n=20)$

\begin{tabular}{|c|c|c|c|c|c|c|c|}
\hline & 1 & 2 & 3 & 4 & 5 & 6 & 7 \\
\hline 1. $\mathrm{HCS}^{\mathrm{a}}$ & 1.00 & $.53 *$ & $.61 * *$ & $.53 *$ & -.14 & -.01 & .16 \\
\hline 2. ACES & & 1.00 & $.61 * *$ & $.65 * *$ & .07 & .32 & .42 \\
\hline 3. PSWQ-PW & & & 1.00 & $.85 * *$ & .01 & .29 & .42 \\
\hline 4. WAQ-A-PW & & & & 1.00 & -.06 & $.53 *$ & .35 \\
\hline 5. CES-D & & & & & 1.00 & -.13 & .36 \\
\hline 6. IUS & & & & & & 1.00 & $.65 * *$ \\
\hline 7. NPOQ & & & & & & & 1.00 \\
\hline
\end{tabular}

Note. HCS = Homework Compliance Scale; ACES = Anxiety Change Expectancy Scale at baseline; PSWQ-PW = change on the Penn State Worry Questionnaire - Past Week; WAQ-A-PW = change on the Worry and Anxiety Questionnaire-Associated Symptoms Subscale - Past Week; CES-D = change on the Centre for Epidemiological Studies Depression Scale; IUS = change on the Intolerance of Uncertainty Scale; NPOQ = change on the Negative Problem Orientation Questionnaire. Change scores on outcome variables were calculated by subtracting scores on a measure at 1-week post baseline from scores on the same measure at baseline. Two-tailed correlations. ${ }^{\mathrm{a}} n=18$ participants.

${ }^{*} p<.05,{ }^{* *} p<.01$ 
Table 20

Zero-Order Correlations of Homework Compliance and Anxiety Change Expectancy to Change Scores on GAD-Related Outcomes from Baseline to 2-Weeks Post Baseline in the Exposure Condition $(n=20)$

\begin{tabular}{|c|c|c|c|c|c|c|c|}
\hline & 1 & 2 & 3 & 4 & 5 & 6 & 7 \\
\hline 1. $\mathrm{HCS}^{\mathrm{a}}$ & 1.00 & $.53 *$ & $.56 * *$ & .33 & .21 & .33 & .28 \\
\hline 2. ACES & & 1.00 & $.44^{*}$ & .29 & .19 & $.50 *$ & .33 \\
\hline 3. PSWQ-PW & & & 1.00 & $.72 * *$ & .41 & $.52 *$ & $.49 *$ \\
\hline 4. WAQ-A-PW & & & & 1.00 & .33 & $.67 * *$ & $.45^{*}$ \\
\hline 5. CES-D & & & & & 1.00 & $.56 * *$ & $.54 *$ \\
\hline 6. IUS & & & & & & 1.00 & $.82 * *$ \\
\hline 7. NPOQ & & & & & & & 1.00 \\
\hline
\end{tabular}

Note. HCS = Homework Compliance Scale; ACES = Anxiety Change Expectancy Scale at baseline; PSWQ-PW = change on the Penn State Worry Questionnaire - Past Week; WAQ-A-PW = change on the Worry and Anxiety Questionnaire-Associated Symptoms Subscale - Past Week; CES-D = change on the Centre for Epidemiological Studies Depression Scale; IUS = change on the Intolerance of Uncertainty Scale; NPOQ = change on the Negative Problem Orientation Questionnaire. Change scores on outcome variables were calculated by subtracting scores on a measure at 2-week post baseline from scores on the same measure at baseline. Two-tailed correlations. ${ }^{\mathrm{a}} n=18$ participants.

${ }^{*} p<.05,{ }^{* *} p<.01$ 
Daily outcomes. It was also expected that higher scores on homework compliance and anxiety change expectancy would predict lower scores on daily worry time, daily GAD symptoms, and daily IU cognitions overall (main effects) or over 14 days of monitoring (interaction effects) in the exposure condition. Given the limited research, no specific hypotheses were proposed about whether main effects or interaction effects would be found.

HLM analyses were performed for each of the daily outcomes (e.g., daily worry time). In these models, time (i.e., repeated observations over 14 days) was the level 1 predictor, and the two homework-related variables were the level 2 predictors. In each of the three analyses, the covariance structure model that was the most appropriate fit for the data was the random intercept and random slope uncorrelated model.

The results of the HLM analyses are displayed in Table 21. There was no significant main effect of time on any daily outcome, nor were there significant main effects of HCS or ACES scores on any outcomes. However, there was a significant time x HCS interaction effect on daily worry time and on daily IU cognitions, showing that individuals with higher HCS scores reported lower daily worry time $(b=-0.79, t=-2.62, p=.02)$ and lower daily IU cognitions $(b=-0.02, t=3.08, p$ $<0.01$ ) over 14 days. There were no other significant interaction effects. 
Table 21

Multilevel Models for Daily Outcomes as Associated with Time and Homework-Related Variables in the Exposure Condition $(n=18)$

\begin{tabular}{|c|c|c|c|c|}
\hline Daily Outcomes & Fixed effects & $b$ & $S E$ & $t$ \\
\hline Daily Worry & Intercept & 372.95 & 179.31 & 2.08 \\
\hline \multirow[t]{5}{*}{ Time } & Time & 3.85 & 15.30 & 0.25 \\
\hline & HCS & -1.56 & 3.37 & -0.46 \\
\hline & ACES & -2.48 & 2.09 & -1.19 \\
\hline & Time*HCS & -0.79 & 0.30 & $-2.62 *$ \\
\hline & Time*ACES & 0.29 & 0.18 & 1.56 \\
\hline Daily GAD & Intercept & 7.49 & 3.33 & $2.25^{*}$ \\
\hline \multirow[t]{5}{*}{ Symptoms } & Time & -0.04 & 0.31 & -0.13 \\
\hline & HCS & -0.04 & 0.06 & -0.61 \\
\hline & ACES & -0.02 & 0.04 & -0.43 \\
\hline & Time*HCS & -0.006 & 0.006 & -0.99 \\
\hline & Time*ACES & 0.003 & 0.004 & 0.67 \\
\hline Daily IU & Intercept & 7.05 & 3.74 & 1.88 \\
\hline \multirow[t]{5}{*}{ Cognitions } & Time & -0.005 & 0.26 & -0.02 \\
\hline & HCS & -0.003 & 0.07 & -0.04 \\
\hline & ACES & -0.03 & 0.05 & -0.72 \\
\hline & Time*HCS & -0.02 & 0.005 & $-3.08 * *$ \\
\hline & Time*ACES & 0.006 & 0.003 & 1.94 \\
\hline
\end{tabular}

Note. HCS = Homework Compliance Scale scores; ACES = Anxiety Change Expectancy Scale scores at baseline. $n=18$ as two participants did not complete the HCS.

${ }^{*} p<.05, * * p<.01$ 


\section{Discussion}

The present experiment tested whether practicing uncertainty exposure produces the positive effects that it is theorized to have on GAD symptoms, intolerance of uncertainty (IU) and negative problem orientation. The present study also investigated the degree to which homework compliance and anxiety change expectancy were associated with improvement on GAD symptoms, IU, and negative problem orientation over the course of uncertainty exposure.

\section{Effects of Uncertainty Exposure on GAD Symptoms}

Individuals who completed uncertainty exposure reported significant medium reductions in weekly worry and GAD associated symptoms (e.g., muscle tension, fatigue) from baseline to 1-week post baseline, and worry remained significantly reduced at 2-weeks post baseline. In contrast, people in the control condition reported negligible to small nonsignificant changes in weekly worry and GAD-associated symptoms from baseline to 1- and 2-weeks post baseline. At 1-week post baseline, people in the exposure condition also showed significantly lower worry and GADassociated symptoms than did people in the control condition. However, the between-condition differences in GAD symptoms were no longer present at 2-weeks post baseline, as the control condition reported a significant medium improvement in weekly worry between 1- and 2-weeks post baseline, and a nonsignificant small improvement in weekly GAD-associated symptoms between these time points. At the daily level, individuals who completed uncertainty exposure reported significantly less time spent worrying and lower GAD symptom severity than did individuals in the control condition. Taken together, these results suggest that uncertainty exposure training and 1week of uncertainty exposure homework has positive effects on GAD symptoms. It is of note that people in the assessment only control condition reported changes in weekly worry between the post baseline time points, and small nonsignificant improvements in weekly GAD-associated symptoms over time; these findings are considered further later in the Discussion. 


\section{Effects of Uncertainty Exposure on IU and Negative Problem Orientation}

Intolerance of uncertainty. IUS scores improved significantly with medium effects from baseline to 2-weeks post baseline in people who did uncertainty exposure, but remained unchanged in people who did assessment only. In addition, the results showed that in the exposure condition, improvement on the IUS accounted for significant unique variance in weekly worry and GADassociated symptoms at 1- and 2-weeks post baseline, controlling for baseline levels of GAD symptoms. On the other hand, in the control condition, change in IU was unrelated to GAD symptoms at both post baseline assessments, controlling for baseline GAD symptoms levels. These findings are consistent with theory underlying cognitive behavioural treatment focused on IU (Dugas \& Robichaud, 2007; Dugas et al., 2010) which proposes that manipulating a person’s approach to uncertainty in their daily life via uncertainty exposure may improve GAD symptoms and IU, and that changes in GAD symptoms and IU are related. In other words, over the course of uncertainty exposure, improvement in IU may account for some of the reduction in GAD symptoms.

The present findings show that targeting IU in people with GAD using a CBT strategy improves GAD symptoms. These results extend prior studies demonstrating that experimentally manipulating IU in nonclinical student samples leads to corresponding changes in worry (Ladouceur et al., 2000; Meeten, Dash, Scarlet, \& Davey, 2012; Rosen \& Knäuper, 2009). The current findings represent a novel piece of evidence consistent with the IU Theory of GAD (Dugas et al., 1998), a model that conceptualizes IU as the key factor that underlies the development and maintenance of pathological worry. In addition, the current finding that reversing behavioural attempts to avoid or minimize uncertainty improves GAD symptoms points to the possibility that behavioural manifestations of IU (e.g., avoidance behaviour) may play a specific role in GAD maintenance. Thus, it may be useful to further test and potentially add behavioural manifestations of IU to Dugas et al.'s (1998) original cognitive model of GAD. Clinically, these findings support the usefulness of 
talking to GAD clients about how IU contributes to worry and avoidance (Dugas \& Robichaud, 2007).

Another factor to consider in interpreting the findings is that the exposure condition received psychoeducation about uncertainty, including a discussion of the inevitability of uncertainty in dayto-day life (Dugas \& Robichaud, 2007). This psychoeducation, and perhaps other aspects of uncertainty exposure (e.g., approaching uncertainty in a predictable, systematic manner) may have helped individuals in the exposure condition to come to see uncertainty as more predictable; in other words, something to expect. Theory and research in humans and animals has long suggested that predictable aversive events produce less anxiety than unpredictable events (e.g., Abbott, 1985; Fanselow, 1980; Grillon, Baas, Lissek, Smith, \& Milstein, 2004; Grillon et al., 2008). Thus, although change in the degree to which uncertainty was perceived as predictable and expected were not measured in the present study, it could be theorized that if uncertainty exposure helped people to view uncertainty as expected to a greater extent, then this may have contributed to less negative responses to uncertainty, including less worry when uncertain.

In addition to assessing the effect of uncertainty exposure on the IUS, this study assessed its effect on daily IU cognitions over 2-weeks using a diary method. The results showed that daily IU cognitions did not differ significantly in the exposure compared to control condition, even though the exposure condition reported lower overall daily GAD symptoms than the control condition. In order to interpret these findings, the manner in which daily outcomes were assessed warrants consideration. The items used to assess daily GAD symptoms had either been used in prior research (e.g., daily time spent worrying; Ladouceur et al., 2004; Verkuil et al., 2007) or were very similar to a validated measure of GAD symptoms (the WAQ). But due to the lack of research on daily IU cognitions, and the length of validated measures of IU, the items assessing IU cognitions on the 
present study's daily assessment were based on theory and the underlying factors of the IUS (e.g., uncertainty has negative personal implications, uncertainty is unfair; see Sexton \& Dugas, 2009). Indicators of reliability and validity (see Measures section) suggested that the daily GAD symptoms assessment had high internal consistency and was significantly correlated with validated measures of GAD symptoms. On the other hand, although the daily IU cognitions assessment showed adequate internal consistency, it was not dependably associated with the IUS. These factors point to a potential measurement issue with the present daily IU cognitions scale, and highlight the need for future research to develop validated daily measures of cognitive processes such as IU. Another possibility is that daily IU cognitions were adequately assessed in the present study, but IU cognitions fluctuate erratically at the daily level, particularly in the midst of uncertainty exposure, making improvement difficult to detect. Indeed, daily GAD symptoms are significantly more erratic over time than are trait GAD symptoms (Joos et al., 2012), suggesting that variables measured at the daily level may fluctuate to a greater degree than variables measured at the dispositional level.

Negative problem orientation. Given that problems are inherently uncertain, and IU is strongly related to a negative attitude toward problems and the problem solving process (negative problem orientation [NPO]; $r=.76, p<.001$ in the present study at baseline; Dugas et al., 2007; Robichaud \& Dugas, 2005), the present study examined the degree to which uncertainty exposure impacts NPO. The results demonstrated that in individuals who systematically approached uncertainty, NPO improved to a significant and large extent from baseline to 2-weeks post baseline, whereas NPO remained unchanged in individuals who did assessments only. As with IU, this study assessed whether improvement in NPO was related to GAD symptoms after 1 or 2 weeks of exposure training, and found that in the exposure condition, improvement in NPO was a significant unique predictor of weekly worry at 2-weeks post baseline, controlling for baseline levels of weekly 
worry. Thus, it seems that the large improvement in NPO observed in individuals who did uncertainty exposure was significantly related to worry after two weeks, suggesting that NPO could be another mechanism through which uncertainty exposure effects positive change in GAD symptoms. Contrary to expectations, in the control condition improvement in NPO was also a significant unique predictor of weekly worry at 1-week post baseline, controlling for baseline weekly worry. Thus, even though NPO did not change for people in the control condition on average, in instances where NPO did improve, it explained significant variance in worry levels at 1-week post baseline.

Given the close relationship between IU and NPO, these cognitive processes may interact during the course of uncertainty exposure to lower GAD symptoms. Indeed, the strong significant correlations of changes in IU scores to changes in NPOQ scores at both post baseline assessments in the present study ( $r \mathrm{~s}=.65$ and .82 ) support the idea that these may be overlapping mechanisms through which uncertainty exposure improves GAD symptoms. It may be that as people gained experience with approaching uncertainty, they also become more comfortable with approaching problems, which by nature contain many uncertain elements (e.g., uncertainty about the best possible solution, uncertainty about when the problem will resolve). Stated differently, tolerance for problems may be just one aspect of tolerance for uncertainty, potentially explaining why these constructs improved together.

Similar to the notion that difficulty tolerating problems may be one part of difficulty tolerating uncertainty, it was considered that planning and executing uncertainty exposures was, in itself, a problem solving exercise. During uncertainty exposure, individuals identified the ways in which not being able to withstand uncertainty was playing out in their day-to-day lives, and in many cases, the manifestations of IU that people recognized were current problems. For example, putting 
off activities (e.g., school projects, health appointments), avoiding activities with uncertain outcomes (e.g., applying for jobs), checking over and perfecting work to the detriment of productivity, or seeking reassurance to the point of strained relationships. Next participants worked with the experimenter to brainstorm realistic, manageable, concrete ways to begin to reverse these behaviours over the coming week. This included plans to spend realistic amounts of time engaging in activities with uncertain outcomes that were being delayed or avoided, and plans to reduce checking or reassurance seeking. Experience with a brief “version” of problem solving in this manner, including practice with setting realistic, attainable goals and solution implementation each day, may have helped individuals in the exposure condition to improve their attitude toward problems, thereby contributing to reduced worry.

Given the finding that uncertainty exposure improves problem orientation in people with GAD to a large degree, future research may seek to understand what CBT strategies are most helpful in improving a negative attitude toward problems. To this end, the effects of uncertainty exposure on negative problem orientation could be compared to the effects of traditional problem solving training, another module of CBT-IU for GAD. Indeed, individuals with GAD have a negative attitude toward problems which interferes with problem solving (Davey, 1994; Dugas et al., 1998), but do not lack knowledge of problem solving skills (Dugas et al., 1997; Ladouceur et al., 1998), potentially supporting a hypothesis that people with GAD would benefit more from exposure to the uncertainty inherent in problems versus learning problem solving steps, although this hypothesis awaits empirical testing. Additional research is also needed to discern the essential CBT strategies that assist individuals with GAD, given the many benefits of moving toward more parsimonious treatment protocols (e.g., ease of clinician training; Cougle, 2012). 


\section{Potential Mechanisms of Change beyond the IU Model}

Behavioural activation. It is also of note that uncertainty exposure not only led to reduced GAD symptoms, but also produced significant, medium to large improvements in depressive symptoms from baseline to 1- and 2-weeks post baseline, whereas there were nonsignificant negligible to small changes in depressive symptoms in the control condition. Depressive symptoms may have improved in the uncertainty exposure condition as a by-product of improved GAD symptoms, or vice-versa, as psychological symptoms often improve together (Norton et al., 2013). Along these lines, it is of note that several GAD-associated symptoms overlap with symptoms of depression, including: difficulty sleeping, difficulty concentrating, fatigue and restlessness/psychomotor agitation (APA, 2013). Thus improved GAD-associated symptoms may be difficult to distinguish from improved depressive symptoms because of shared symptomatology. Alternatively, the reduction in depressive symptoms may have been a result of the uncertainty exposure technique itself, which upon consideration, resembles behavioural activation, an evidencebased treatment for depression (see Cuijpers, van Straten, \& Warmerdam, 2007). Behavioural activation involves scheduling activities each day that have the potential to provide a sense of accomplishment or pleasure. The practices that individuals in the present study planned had the potential to provide a sense of accomplishment, as the behaviours often involved taking manageable steps toward addressing problems. As individuals began to take action, this may have led to feelings of mastery, perhaps improving depressive symptoms.

Expectations. It is well documented that expectations for change can produce actual improvement in outcomes, known as the placebo effect (see Price, Finniss, \& Benedetti, 2008). The mean level of anxiety change expectancy in the present sample as measured by the ACES was above the mid-point of the scale, but below the top quartile of the scale, and there were no differences in expectancy for anxiety change between the conditions at baseline or either post baseline assessment. 
This suggests that people in the exposure and control conditions had medium to high expectations for change. Baseline anxiety change expectancy scores in the present study were comparable to those found in prior research of GAD samples who were aware that they would learn CBT $(M=$ 71.69, $S D=12.48$; see Westra et al., 2007). The medium to high expectations for change may have been produced in both conditions by the knowledge that they would learn a technique that may be helpful for worry, either immediately or following the study. Indeed, people in the control condition learned that they too could receive uncertainty exposure following other study components at the time of informed consent, and most people in the control condition requested the training after the study. Given that the exposure and control conditions had comparable expectations for anxiety change, it seems that expectations were not the only factor influencing the positive effects found in people who did uncertainty exposure.

Reactive effects of self-monitoring. Individuals in both conditions completed three batteries of questionnaires in the lab as well as daily self-monitoring for 2 weeks. Although this assessment procedure alone did not produce positive effects to the same degree as assessment plus uncertainty exposure, it nonetheless led to small improvements in weekly GAD symptoms. These findings are consistent with studies showing that self-monitoring can lead to small positive changes in the frequency of target behaviours, thoughts, or feelings, known as the reactive effects of selfmonitoring (see Korotitsch \& Nelson-Gray, 1999). For this reason, self-monitoring itself is sometimes considered an intervention, and it is used as part of many evidence-based CBTs for anxiety disorders, as well as other problems (Humphreys, Marx, \& Lexington, 2008). It may be that self-monitoring GAD-related symptoms and processes increased individuals' awareness of their difficulties, potentially leading to efforts to address them (e.g., reduce time spent worrying). Indeed, the idea that people may have engaged in more problem solving to reduce GAD symptoms after they 
became aware of them is in line with the present finding that improvement in NPO was associated with reduced worry in the control condition. It is also of note that the reactive effects of selfmonitoring may have contributed to the improvement in GAD symptoms and processes that was observed in the uncertainty exposure condition as well, as both conditions tracked GAD-relevant symptoms and processes over 2 weeks.

\section{Relation of Homework to GAD Outcomes}

The present study also investigated the association of homework compliance and anxiety change expectancy to GAD-related outcomes at the general and daily level in those who received uncertainty training. The results demonstrated that completing more homework was associated with greater improvement in weekly worry at 1-and 2-weeks post baseline, and GAD-associated symptoms at 1-week post baseline. Similarly, having higher expectations that anxiety can reduce was related to greater improvement in weekly worry at 1- and 2-weeks post baseline, GADassociated symptoms at 1-week post baseline, and IU at 2-weeks post baseline. At the daily level, homework compliance emerged as a significant unique predictor of improvement in daily time spent worrying and improvement in daily IU cognitions over 2 weeks. These findings suggest that completing uncertainty exposure homework and having more positive expectations for anxiety change may play an important role in producing greater improvement in both GAD symptoms and IU. In other words, it seems that the "dose" of uncertainty exposure could be important. This is consistent with research suggesting that homework compliance and anxiety change expectancies predict outcomes in CBT for GAD (Newman \& Fisher, 2010; Westra et al., 2007), and other anxiety disorders (Price \& Anderson, 2012; Rees, McEvoy, Nathan, 2005).

Clinicians may wish to communicate the importance of completing homework to clients, plan uncertainty exposures collaboratively with clients in session, and ensure that clients understand what specific uncertainty exposures they are to do for homework, as such an approach may facilitate 
greater homework compliance (Leahy, 2005; Waters \& Craske, 2005). In the present study, participants were instructed to complete one uncertainty exposure practice per day, and each exposure was planned collaboratively in the lab with the experimenter. In addition, the experimenter checked participants' understanding of the activities that they were to carry out, and clarified when participants were unsure. The current approach seemed to produce good homework compliance and positive effects over a short timeframe. Given the strong homework compliance found in this study (see Results), the “one per day” exposure guideline appears attainable for clients, and might serve as a reasonable heuristic for clinicians until future research can provide more precise guidelines on the optimal parameters of uncertainty exposure.

\section{Limitations and Future Directions}

The present treatment experiment addressed a novel research question about the effects of uncertainty exposure on GAD symptoms and IU-related cognitions at the general and daily level, assessed how homework and anxiety change expectancy relate to outcomes with uncertainty exposure, and provided new evidence to support and extend the IU model of GAD. However, there are limitations to consider. One is the way in which daily IU cognitions were assessed, as there is not presently a validated measure of daily IU cognitions, and the items that were developed for this

study showed an inconsistent association with the IUS. Future research is needed to develop a daily measure of relevant GAD constructs, so that change can be more accurately investigated at the momentary level, which would improve the current research on mechanisms of change. The present study’s assessment of daily constructs provides a starting place for future research endeavours, although additional refinement of the current daily IU cognitions assessment is needed.

Another limitation of the present study is that although it included several measures of constructs related to the main hypotheses (e.g., general and daily GAD symptoms), the current study 
assessed constructs primarily from the IU Theory of GAD (Dugas et al., 1998), given that the technique being tested involved confronting uncertainty. This could be built on in future studies of uncertainty exposure through the measurement of constructs from other well-supported GAD models (e.g., questionnaires on emotion regulation or metacognition). Indeed, assessing constructs from several GAD models may provide new insights about how components of these theories converge.

It is also possible that the study was underpowered due to the sample size. In addition, a nontreatment-seeking sample of individuals who met diagnostic criteria for GAD were recruited, and there may be differences between nontreatment-seeking community populations and treatmentseeking populations. Thus, uncertainty exposure should be tested in treatment-seeking samples to assess the generalizability of the present results. Related to generalizability, it is of note that mean baseline levels of worry and IU in the present study were higher than those reported in clinical trials (Dugas et al., 2003, 2010; Ladouceur et al., 2004; van der Heiden et al., 2012; Wells et al. 2010). This may have impacted the results in either a positive or negative direction. For instance, it is possible that people with more severe symptoms are more willing to engage in a treatment strategy in order to gain some relief; although it is equally conceivable that more severe symptoms (e.g., difficulty concentrating) and cognitive processes (e.g., very high IU) could interfere with learning and carrying out uncertainty exposure. Future research is needed to test whether uncertainty exposure has the same effects in treatment-seeking samples, and across people with varying levels of GAD symptoms and IU.

Given that this is the first known investigation of uncertainty exposure and that the results were promising, future research should focus on how to optimize the effects of uncertainty exposure. For example, the present findings indicated that more homework was associated with greater improvement on daily worry and daily IU cognitions. Future studies could determine what "dose” of 
uncertainty exposure is needed to produce optimal outcomes, and relatedly, how many experiments individuals should carry out between therapy sessions. Its limitations notwithstanding, the present study provided the first test of the impact of uncertainty exposure on GAD symptoms and several cognitive processes using an approach designed to approximate how uncertainty exposure is delivered in clinical practice. The current study assessed outcomes at two time points and considered constructs such as anxiety change expectancy, which helped clarify factors contributing to the present findings.

\section{Summary and Conclusion}

The present study investigated a CBT technique for GAD involving systematically approaching uncertain situations and the feelings that this evokes compared to an assessment only control condition. Confronting uncertainty and related feelings produced medium to large positive effects on GAD symptoms, depression, intolerance of uncertainty, and negative problem orientation over the course of two weeks. The results also showed that improvement on intolerance of uncertainty and negative problem orientation were related to improved GAD symptoms, supporting these cognitive processes as potential mechanisms of change with uncertainty exposure. Completing more homework and having positive expectations that one’s anxiety can improve were associated with more positive outcomes in people who did uncertainty exposure. Overall, the findings provide additional support for Dugas et al.’s (1998) IU Theory of GAD by showing that repeatedly approaching uncertain situations or refraining from behaviours that reduce uncertainty improve GAD symptoms and related cognitive processes. 


\section{General Discussion}

The present dissertation investigated unanswered questions about attitudes toward uncertainty and behavioural responses to uncertainty in people with GAD compared to nonpsychiatric control participants. This thesis also tested the short-term effects of a treatment technique called uncertainty exposure on GAD symptoms and cognitive processes. Broader implications of the findings are explored below, including a discussion of how IU might be conceptualized in light of the current results, as well as ideas for refining the current treatment of IU in people with GAD.

\section{Responses to Uncertainty as an Approach-Avoidance Conflict}

An "approach-avoidance conflict” refers to a state where a goal or event is simultaneously appealing and unappealing (Lewin, 1935; Miller, 1944). An example of an approach-avoidance conflict might be the decision to pursue a new job if one is dissatisfied with their current job. On the one hand, pursuing new work may be appealing due to the potential for greater satisfaction, and possibly a sense of relief if one leaves an unfavourable situation. On the other hand, pursuing new work may be simultaneously unappealing due to factors such as high effort required to switch jobs or the potential risk of being more even dissatisfied with a new job than one's current job. Approachavoidance conflicts can be highly stressful (Boyd, Robinson, \& Fetterman, 2011; Miller, 1944; Robinson \& Gordon, 2011) and produce behavioural “dithering” in animals, wherein animals go back and forth between seeking out a reward and moving away from it (e.g., McNaughton, 2011).

Early theorizing on GAD and worry suggested that people with GAD may be both intolerant of uncertainty and intolerant of emotional arousal (Dugas \& Ladouceur, 1998; Krohne, 1993). Krohne (1993) hypothesized that anxious individuals may engage in hypervigilant behaviours in order to increase their level of certainty, and at the same time, may try to avoid threatening information that provokes anxiety. Consistent with this, Dugas, Freeston, Doucet, Provencher, and 
Ladouceur (1995) showed that people with higher levels of worry reported elevated IU and intolerance of emotional arousal. Dugas and Koerner (2005) later theorized that high IU may compel people with GAD to approach uncertainty by engaging in behaviours such as excessively seeking information about concerns of future negative outcomes (e.g., spending a lot of time researching symptoms when worried about whether one has an illness), but when the anxiety associated with uncertainty becomes too overwhelming, people with GAD then use strategies to dampen their arousal, such as distraction. This going back-and-forth between approach and avoidance behaviour is theorized to contribute to and maintain anxiety (Dugas \& Ladouceur, 1998; Krohne, 1993). In sum, the way in which people with GAD cope with uncertainty has been conceptualized as an approach-avoidance dilemma, characterized by competing desires "to know" and "not to know" (Dugas \& Koerner, 2005; Dugas \& Robichaud, 2007).

The findings of studies within the present dissertation build on extant discussions of the approach-avoidance conflict that people with GAD theoretically contend with. In Study 1a of this project, people with GAD offered responses reflecting a stronger desire for certainty than did nonpsychiatric control participants. Those with GAD described thoughts of "needing" to know when uncertain, reported they would choose a sure negative outcome over being uncertain, and described uncertainty as bothersome in and of itself (i.e., being bothered by not knowing). Paradoxically, however, in Study 1a the responses of people with GAD simultaneously reflected a desire "not to know" compared to the answers of non-psychiatric control participants. People with GAD said that uncertainty is difficult to tolerate because they assume the worst will happen, and because of the feelings it causes them. Those with GAD also described being "allergic" to uncertainty, suggesting a need to stay away from unknowns. Overall then, the attitudes toward uncertainty of people with GAD as revealed in Study 1a reflected competing motivations to move toward and away from 
uncertainty, whereas the responses of non-psychiatric control participants did not reflect these themes to the same degree.

The findings of Study $1 \mathrm{~b}$ are similarly consistent with conceptualizing responses to uncertainty in people with GAD as an approach-avoidance dilemma. Study $1 \mathrm{~b}$ showed that most of the behaviours that people with GAD and people without a diagnosis engage in when they are uncertain can be categorized as approach and avoidance responses, and that people with and without GAD use both types of behaviours. Furthermore, people with GAD and non-psychiatric control participants alike said that the behaviours that they engage in when they are uncertain serve the functions of increasing certainty and reducing distress, as has been proposed in theoretical writings on the approach-avoidance dilemma in people with GAD (e.g., Dugas \& Koerner, 2005). Thus, it seems that using approach and avoidance behaviours when uncertain is not pathological in itself, as this was described by people without a psychological disorder as well as those with GAD. However, the difference between people with GAD and non-psychiatric controls may lie in how frequently they go "back-and-forth" between approach and avoidance behaviours, as people with GAD described using both types of behaviours significantly more often to cope with uncertainty than did non-psychiatric control participants. Future research is needed to test this potential difference that may characterize the approach-avoidance behaviours of people with GAD, as the present study did not assess how frequently people with GAD change their style of dealing with uncertainty from seeking out a sense of sureness to pulling away from knowing.

Study 2 of this dissertation demonstrated that helping people with GAD to refrain from excessive engagement in approach behaviours, and to take manageable steps toward previously avoided uncertain situations lead to improvement in GAD symptoms and cognitive processes. As such, it seems that identifying approach and avoidance behaviours relevant to a person, and helping 
them to make a plan to reduce the use of both types of behaviours may be one way to address the approach-avoidance conflict in people with GAD. Indeed, assisting people with GAD to balance acting on the urge to know as well as the desire to avoid may have led to less "back-and-forth" type behaviour. Using uncertainty exposure, people in Study 2 were helped to engage in organized behaviour that involved reducing unnecessary or excessive measures that individuals were taking to be certain (e.g., excessive checking or excessive information seeking) and adding steps toward attaining meaningful goals despite the anxiety associated with moving forward (e.g., spending small amounts of time looking for jobs or trying new activities with friends). In sum, the findings of this dissertation are consistent with earlier theorizing that people with GAD may have competing desires to achieve certainty and to simultaneously avoid it, and that uncertainty exposure may be a way to help people reduce the behaviours associated with this theorized approach-avoidance conflict.

\section{New Insights about the Properties of IU}

IU as continuous. A key take-home message of the present research is that there remains much to discover about the phenomenology of IU. The current results point to the idea that IU is not a pathological process. Several aspects of what people with GAD reported about their thoughts and behaviours when uncertain were also discussed by non-psychiatric control participants. All individuals desire certainty to some degree, and have concerns about potential negative outcomes. Similarly, all people reported engaging in attempts to manage uncertainty, and in fact, those with and without GAD generally reported using the same behaviours when uncertain. However, there were negative beliefs about uncertainty and the uncertain self that were present only in people with GAD, and the frequency of behavioural responses to uncertainty was higher in people with GAD compared to individuals without a psychiatric diagnosis. In addition, people with GAD and non-psychiatric control participants both said that they engage in behaviours when uncertain for several similar 
reasons - including to increase certainty and to reduce distress. These findings support the idea that people are motivated to know (see Al-Najjar \& Weinstein, 2009; Rosen et al., 2007) and that uncertainty is at least somewhat uncomfortable to the vast majority of people (Curley et al., 1986; Epstein, 1999; Fox \& Tversky, 1995; Inglis, 2000; Montesano, \& Giovannoni, 1996). In sum, the findings of this dissertation suggest that most individuals are to some extent intolerant of uncertainty.

The current data support recent recommendations that IU be conceptualized as a continuous construct, present in all people to a degree (Birrell et al., 2011; Carleton et al., 2012). Considering IU on a continuum is consistent with the recent paradigm shift proposed in DSM-5 (APA, 2013); that the field of clinical psychology move toward conceptualizing symptoms of psychiatric disorders as dimensional as opposed to categorical (e.g., Adam, 2013; Frances \& Widiger, 2012; Jones, 2012; Narrow \& Kuhl, 2011; Regier, Kuhl, \& Kupfer, 2013; Regier, Narrow, Kuhl, \& Kupfer, 2009; Widiger, 2011). Thinking along a continuum when it comes to mental health may extend well beyond considering symptoms as dimensional, to considering cognitive processes and behaviours related to psychological symptoms as continuous as well. Indeed, there are several examples of this in prior research, including perfectionism (Broman-Fulks, Hill, \& Green, 2008) and compulsive behaviours (e.g., Mataix-Cols, do Rosario-Campos, \& Leckman, 2005). Conceptualizing processes such as IU as continuous may lead to further study of aspects of IU that are typical of people, potentially facilitating greater normalization of common reactions to uncertainty in the treatment of clients with high IU. Framing IU as continuous may also lead to the development of interventions that more specifically target unhelpful aspects of IU, while not challenging functional components (i.e., not treating what is normal). 
IU as multidimensional. An important realization that was made after analyzing the data of the present studies was that it is still unknown what is actually meant by "uncertainty" when researchers refer to the construct "intolerance of uncertainty." There has been limited discussion to date in the GAD literature about whether intolerance of uncertainty refers to intolerance of the feeling of being unsure, or to intolerance of situations with several different outcomes, or perhaps both. In early writings, IU was described as the way an individual perceives information in ambiguous situations, and responds to this information with a set of cognitive, emotional, and behavioural reactions (Dugas et al., 1998; Freeston et al., 1994; Ladouceur et al., 1997). From my perspective, it is not entirely clear whether the uncertainty referred to in these descriptions is that of internal unsure feelings, external ambiguous situations, or both. Thus, to the best of my knowledge, the form of "uncertainty" that intolerance of uncertainty refers to has not yet been clearly defined, and there remains to be empirical investigations on this topic in the GAD literature.

Outside of research on IU in GAD, nonclinical research has pointed out that there may be a difference between internal and external attributions of uncertainty (e.g., Howell, 1971; Kahneman \& Tversky, 1982; Teigen, 1994). According to Kahneman and Tversky (1982), internal uncertainty refers to the subjective experience of being hesitant about one’s own state of knowledge (e.g., being unsure about how one did on an exam, or not knowing what to do), whereas external uncertainty refers to objective unknowns in the external world (e.g., what the weather will be like tomorrow, or the outcome of a coin toss); the difference lies in the source of a person's uncertainty. There is also some evidence from the decision making literature that individuals are generally more confident about outcomes when the source of uncertainty is internal versus external, potentially because nonclinical individuals feel like they have more "control” over uncertainty with an internal source (Howell, 1971; Howell \& Burnett, 1978). In sum, outside of the clinical psychology literature a 
distinction has been made about different types of uncertainty, and people may respond differently depending on the type of uncertainty in question.

The findings of the present dissertation might be interpreted to suggest that people with GAD are intolerant of both internal and external uncertainty. People with GAD and non-psychiatric control participants provided information suggesting that they are at least somewhat intolerant of external uncertainty. People with GAD said that uncertainty is negative because they make the assumption that events with many potential outcomes will resolve badly, and non-psychiatric control participants described this idea in less absolute terms, saying that uncertainty can be negative in some cases because it can resolve poorly. These findings suggest that it is generally uncomfortable for all individuals to not know what will happen due to the possibility of negative outcomes. However, the assumptions of people with GAD may make it even more difficult to face scenarios with many possible eventualities, and hence, may make them more intolerant of external uncertainty.

Findings consistent with the idea that people with GAD are intolerant of internal uncertainty include that people with GAD said that uncertainty is intolerable because of how it makes them feel, and described experiencing negative beliefs about themselves when uncertain (e.g., "I’m incompetent”, “I'm abnormal”), suggesting that being unsure is a negative, personal experience to people with GAD. Moreover, people with GAD said that they were bothered by not knowing in and of itself, in addition to not knowing about potential negative outcomes. Taken together, my interpretation of these findings is that people with GAD may have difficulty with their own reactions to uncertainty, or in other words, may be intolerant of the internal experience of being unsure and not simply the possibility of situations resolving negatively. Being intolerant of internal uncertainty may be an aspect of IU that is more pathological, as there were indications that this could be present in people with GAD, but no such indications in people without a psychological disorder. However, 
experiences of internal and external uncertainty were not specifically measured in the present study, and it is possible that people were thinking of one type of uncertainty or the other when providing responses; thus, it is difficult to make accurate interpretations about "internal” versus “external" uncertainty. More precise testing of the hypothesis that people with GAD may be intolerant of external and internal uncertainty, and how this may differ from non-psychiatric controls is needed before conclusions are drawn. If individuals with GAD are intolerant of the internal felt sense of uncertainty, in addition to situations with unknown outcomes, then it may be important for therapists to help clients attend to the internal felt sense of being uncertain during exposure in order to facilitate new learning about one's ability to cope with internal uncertainty.

In addition to the present data leading to the observation that there may be external and internal sources of uncertainty, the present project also specifically examined people's attitudes toward some different types of uncertainty, and provided evidence that uncertainty is not a unitary construct to people with or without GAD. People with GAD and non-psychiatric control participants said that uncertainty is more tolerable when they have greater control over it, and when they are not the only one who is uncertain. Some people with GAD and all individuals without a psychological diagnosis said that their attitude toward uncertainty would be less negative in cases where potential negative outcomes were less serious. In addition, people with GAD said that they can feel positive emotions when faced with some kinds of uncertainty, for example, when uncertainty involves only positive outcomes. Overall, the current results suggest that some factors may moderate attitudes toward uncertainty in people with GAD, implying that not all uncertainty is equally intolerable to those who worry excessively. 


\section{Implications of a More Dynamic Conceptualization of IU for Uncertainty Exposure}

Since the present findings indicate that people with GAD respond more or less negatively depending on the parameters surrounding uncertainty, I proposed that confronting different types of uncertainty during exposure could be important, and may improve the effects of uncertainty exposure on GAD outcomes. This hypothesis is based on several principles suggesting that approaching various forms of a feared object or situation maximizes the benefits of exposure (Craske et al., 2014). Taking the example of a person with a specific phobia of dogs, confronting different types of dogs, such as dogs of different colours, sizes, and breeds, as well as approaching dogs across different contexts, for example, approaching dogs with others, alone, indoors and outdoors, all serve to enhance learning and minimize the chance that fears will spontaneously return following successful exposure treatment (Balooch, Neumann, \& Boschen, 2012; Bjork \& Bjork, 2006; Bouton, 1993; Rescorla, 2006; Schmidt \& Bjork, 1992; Vansteenwegen et al., 2007). Indeed, experience with different forms of a feared object or situation across a variety of contexts should help a person learn that fears do not manifest regardless of the specific features of the feared object or situation (e.g., Bjork \& Bjork, 2006; Schmidt \& Bjork, 1992), and exposure to the feared object or situation across different contexts assists with recall at later times (Bouton, 1993; Vansteenwegen et al., 2007). Overall, the finding that people with GAD are bothered by a number of different "types" of uncertainty - for instance, uncertainty with a low likelihood of negative outcomes, uncertainty with a high likelihood of negative outcomes, and uncertainty in the absence of negative outcomes (i.e., “uncertainty itself”) - suggest that exposure to uncertainty could be varied for people with GAD, as is done in the treatment of individuals with other anxiety disorders (see Abramowitz et al., 2011; Craske et al., 2014). 
Furthermore, the idea that people with GAD have different attitudes toward different types of uncertainty could allow for exposure to be adjusted to an appropriate level of "difficulty." The most widely studied approach to exposure is a systematic, stepped approach wherein an individual gradually "works up to" the most feared object or situation (see Abramowitz et al., 2011; Clark, 2004). An example would be a person with a fear of public speaking who engages in exposure by delivering speeches in front of groups that are of increasingly larger size, and on topics of increasingly greater difficulty. There is some research suggesting that exposures work best when they are moderately anxiety provoking (Foa \& McNally, 1996; Otto \& Hofmann, 2009), as this increases the chance that individuals will be able to take in new information about a previously avoided situation (Foa, Huppert, \& Cahill, 2006; Whelton, 2004) without being so anxious that it disrupts learning and memory consolidation (see Moscovitch, Antony, \& Swinson, 2009; Foa \& McNally, 1996). The finding that people with GAD are bothered less by some types of uncertainty than others suggests that uncertainty exposure might be done in a gradual manner with people with GAD. The current results offer ideas for clinicians about factors that may modify how anxiety provoking an uncertain situation is likely to be to a person, such as the degree of control a person has over its outcomes or whether the person will be the only one uncertain in a situation. It should also be considered that resisting the use various behaviours that quell feelings of uncertainty (i.e., "safety behaviours”; e.g., reassurance-seeking, excessive information-seeking, excessive checking, overpreparing), may also be more or less difficult for individuals to do. Thus, another potential way to make uncertainty exposure gradual for a person may be to help them experience uncertainty by slowly refraining from engaging in "safety behaviours" that are increasingly difficult for them to do without. Although the present study provides initial ideas about potential ways to modify the difficulty of uncertainty exposure, several other factors may moderate people's responses to 
uncertainty and could be investigated in future research. As this line of research progresses it may be useful to develop an interview for clinical settings that assesses: 1) reactions to different types of uncertainty, and 2) behaviours that people use to minimize or avoid uncertainty, so that clinicians may better understand how IU manifests for a person and how best to help him or her engage in exposure to uncertainty.

\section{Can We Move Beyond Tolerating Uncertainty?}

Currently, CBT-IU emphasizes learning how to “tolerate” uncertainty, but the idea of enjoying or making the most of uncertainty has to the best of my knowledge not been discussed in the GAD literature. The present dissertation showed that people with GAD have a negative attitude toward uncertainty, and feel negative emotions in response to even uncertainty with positive or neutral outcomes. Nevertheless, this dissertation also presents a number results that reflect the idea that uncertainty may have a bright side. The results of Study 1a showed that people with GAD and non-psychiatric control participants said that uncertainty can be positive in some situations, including when uncertainty is exciting, has positive consequences, produces feelings of freedom, or leads to new learning. Furthermore, like people without a psychological disorder, people with GAD said that they experience positive emotions (albeit, for people with GAD this is alongside negative emotions) when faced with scenarios that involve unknown but positive outcomes, such as not knowing what they will receive as a gift. On the other hand, Study $1 \mathrm{~b}$ of this dissertation revealed that non-psychiatric control participants reported frequently "embracing” uncertainty, which in this case referred to enjoying and making the best of uncertainty, whereas people with GAD said that they rarely embrace uncertainty. Overall these findings suggest that it may be possible for people with GAD to consider some uncertain situations in a favourable light, and offer the idea that it is conceivable to "embrace” uncertainty. 
In a recent article on maximizing anxiety reduction during exposure treatment, Craske and colleagues (2014) highlighted that people learn more when there are larger discrepancies between what they expect will occur when approaching a feared object or situation and what actually occurs (Baker et al., 2010; Deacon et al., 2013; Rescorla \& Wagner, 1972). Simply put, the more incorrect people are about what they think will happen in a feared situation, the more their fear should decrease. As such, approaching uncertain situations that are likely to be positive, such as buying a scratch-and-win ticket or asking a friend to plan a night out, could augment learning during uncertainty exposure by maximizing the difference between a person’s negative beliefs about uncertainty and what actually happens in an uncertain situation. Since the idea that uncertainty might sometimes be a good thing to people with GAD has not yet been considered in research, testing whether approaching "positive” uncertainty can bolster gains from uncertainty exposure awaits empirical testing.

Another way to maximize the mismatch between expectancies and outcomes during uncertainty exposure could be to ask people questions following their experience with uncertainty in order to draw their attention to the positive aspects of being uncertain. Currently in CBT-IU people are asked to record their thoughts during and after uncertainty exposure (Dugas \& Robichaud, 2007). This might be expanded on by asking people to: 1) rate positive emotions during exposure, such as their level of excitement or interest; 2) record any benefits of approaching uncertainty (e.g., flexibility, new learning); and 3) record how the experience of being uncertain differed from expectancies. Indeed, it is suggested that asking people questions after exposure can help facilitate memory consolidation (Craske et al., 2014), and asking questions about the good aspects of uncertainty may help people to better retain this information specifically. Given the usefulness of metaphors in therapy to clarify meanings and provide new perspectives on problems (see Blenkiron, 
2005), an additional way to underscore the advantages of uncertainty may be to use metaphors that cast uncertainty in a positive light.

The finding in the present dissertation that people with GAD identified numerous conditions under which uncertainty can be positive could also be capitalized on to enhance motivation to carry out uncertainty exposure. Indeed, identifying the pros and cons of engaging in efforts to change is a powerful treatment strategy that has been used as part of a treatment called motivational interviewing in order to enhance homework compliance and boost treatment gains in people with GAD (e.g., Westra, Arkowitz, \& Dozois, 2009; Westra \& Dozois, 2006) and other difficulties (e.g., Burke, Arkowitz, \& Menchola, 2003; Geller \& Dunn, 2011). Given these findings, as well as the results from the current project showing that greater homework compliance was associated with better outcomes following uncertainty exposure, it is theorized that asking people with GAD about the pros and cons of carrying out uncertainty exposure prior to using this technique may be a way to further increase homework compliance and enhance the beneficial effects of this strategy.

Study $1 \mathrm{~b}$ of this dissertation revealed that non-psychiatric control participants frequently make the best of and enjoy uncertainty, whereas people with GAD do this infrequently. Although it has not yet been studied, this finding raises the question of whether helping people with GAD learn to make the best of uncertainty may actually be an important part of optimizing treatment gains. Dialectical behaviour therapy (DBT), an effective treatment for borderline personality disorder (see Lynch, Trost, Salsman, \& Linehan, 2007) includes "reality acceptance skills" that involve working to fully accept negative situations that cannot be changed (Linehan, 2015). Future researchers may wish to explore the degree to which reality acceptance skills from DBT may be drawn on and used to help people with GAD to "embrace” uncertainty. Drawing from DBT again, a technique called "opposite action" involves engaging in behaviours that are inconsistent with unhelpful emotions 
(e.g., getting active when one is feeling sad; approaching the perceived threat when one is feeling anxious) and engaging fully and completely with the mood-incongruent behaviour (Linehan, 2015). For example, when acting opposite to sadness, then going out for long jog to the best of one's ability would be preferred to going for a short walk half-heartedly. Borrowing from the rationale underlying opposite action, there may be clinical utility in suggesting that people with GAD approach uncertainty fully and completely by doing their best to embrace uncertainty. In sum, it is proposed that using DBT strategies may help people who are intolerant of uncertainty to make the most of being uncertain rather than just tolerate it; however, these are empirical questions that remain to be tested.

\section{Overall Summary and Conclusions}

There is ample evidence that intolerance of uncertainty contributes to the tendency toward excessive worry, the main feature of GAD (see Carleton, 2012). Research has shown that helping people become more tolerant of uncertainty using CBT strategies successfully improves GAD symptoms (Dugas et al., 2003, 2010); although unfortunately about 30\% of individuals with GAD still do not achieve full symptom remission following evidence-based treatment (see Hanrahan, et al., 2013). Continued investigation of unanswered questions about IU in people with GAD has the potential to increase the theoretical understanding of what keeps pathological worry going and to improve the efficacy of current treatment approaches. Since measures of GAD in the literature have been developed from the perspective of clinician-researchers, this dissertation examined attitudes toward uncertainty from the viewpoint of people with GAD and non-psychiatric control participants in Study 1a. The results of Study 1a revealed new information about attitudes toward uncertainty that are specific to people with GAD, confirmed the accuracy of clinical observations that characterize IU, and yielded data suggesting that IU is a continuous, multidimensional construct. 
Given that avoidance and safety behaviours are key elements that maintain anxiety in conceptualizations of other anxiety disorders (e.g., Hofmann 2008), yet little is known about the behaviours of people with GAD, Study 1b involved an in-depth investigation of behavioural responses to uncertainty in people with and without GAD. Study 1b showed that people with GAD often engage in approach and avoidance behaviours when they are uncertain, and that these behaviours cause problems for people with GAD. It was concluded that behaviours in GAD may be important, and further research should consider the role of behaviours in theoretical models of GAD, as well as the GAD diagnostic criteria. Finally, there are several strategies that comprise full CBT treatments for GAD, although there is limited information about whether specific treatment components are having their intended effects. Study 2 of the present project tested a CBT technique called uncertainty exposure that is used to help individuals increase their tolerance for uncertainty in clinical practice. Study 2 showed that uncertainty exposure has positive effects on GAD symptoms and IU-related cognitions, and that engaging in more uncertainty exposure was related to greater improvement on GAD outcomes. In sum, the findings of this dissertation extend what is currently known about the role of IU in GAD and support the use of uncertainty exposure as a way for people with GAD to improve worry and tolerance for uncertainty. The current findings highlighted several insights and future research directions that have the potential to further the current treatments for people who suffer from excessive worry. 


\section{Appendices}

\section{Appendix A - Participant Recruitment Materials}

\section{Study 1a and Study 1b:}

Interview Study - Worriers Needed!

Do you:

-Worry about a lot of different things?

-Worry more than other people you know?

-Find it difficult to control your worrying?

If so, you may be eligible to participate in a research study at Ryerson University.

You will be compensated for your participation if you are eligible. A telephone screen (which participants will not be compensated for) is required to determine eligibility for this study. All inquiries are confidential.

For more information please contact Katie by phone at 416-979-5000 ext. 2182 or by e-mail at caplab@psych.ryerson.ca

Interview Study - Healthy Participants Needed!

Do you:

-Generally manage anxiety and stress well?

-Generally feel happy?

-Generally feel comfortable with uncertainty?

If so, you may be eligible to participate in a research study at Ryerson University.

You will be compensated for your participation if you are eligible. A telephone screen (which participants will not be compensated for) is required to determine eligibility for this study. All inquiries are confidential.

For more information please contact Katie by phone at 416-979-5000 ext. 2182 or by e-mail at caplab@psych.ryerson.ca 


\section{Study 2:}

Uncertainty Training Study - Worriers Needed!

Do you:

-Worry about a lot of different things?

•Worry more than other people you know?

-Find it difficult to control your worrying?

If so, you may be eligible to participate in a research study at Ryerson University. A telephone screen (which individuals will not be compensated for) is required to determine eligibility for this study. All inquiries are confidential.

You will be compensated for your participation if you are eligible. This study will involve 3 visits to Ryerson University, keeping a self-monitoring log, and learning about a strategy related to uncertainty.

This research is being conducted by a Ryerson University graduate student. For more information please contact Katie by phone at 416-979-5000 ext. 2182 or by e-mail at caplab@psych.ryerson.ca 


\section{Appendix B - Qualitative Interview Guide}

\section{Date:}

\section{Participant ID\#:}

\section{INTRODUCTION:}

The purpose of this interview is to obtain a better understanding of how people experience uncertainty. I will be asking you detailed questions about how you think, feel, and act when you are in uncertain situations. There are no right or wrong answers. I would like you to be as honest and open as you can in this interview, although you are always free to not answer specific questions if you do not want to.

There may be times when I ask you about things that you have already discussed in your prior answers. So if we have already talked about a certain theme that I am asking you questions about, we will just clarify your answer, and then move on.

Also, I will ask you throughout the interview if there is anything else that you have to add to your answer. This is not because your answers are wrong or incomplete, but rather because I want to make sure that I get all relevant information from you.

Please let me know throughout the interview if it is not clear what I am asking.

As you know, the interview will take us about 2 hours. If you are feeling tired or would like to take a break at any time, please let me know. We will break for 10 minutes after 45 minutes and after 1.5 hours.

Do you have any questions at this point?

Ok, let's get started.

\section{Broad Responses to Uncertainty (cognitive, behavioural, emotional, physical):}

The first thing I would like you to tell me about is how you typically react in uncertain situations?

Now, I would like you to think of one specific uncertain situation that you've found yourself in recently, where you acted in a way that is typical of you. By uncertain situation I mean a situation where you felt unsure of what to do, or unsure about how something would turn out.

Can you briefly describe the situation? 
(If participant unsure say): For example, being unsure of which option to chose when making a decision, being unsure of how something went at work, or being unsure about how a new relationship will turn out.

Now, as you're describing, uncertain situations can go on over a period of time, weeks or months. Now, when you answer these questions, I'd like you to focus on those times when you were really feeling uncertain about this situation.

What were your feelings, or moods in this situation?

Moods/feelings:

Probe: Which feeling was the strongest for you in this situation?

Probe: If you were to rate these feelings in intensity from 0 (not at all intense) to 10 (the most intensely I have ever had this feeling), how would you rate these feelings? 110

Probe: What was it about the situation that lead you to feel __?

What did you think to yourself in this situation?

Probe: Did you have any thoughts about the uncertainty? $(\mathrm{y} / \mathrm{n})$

Probe: Did you have any thoughts about yourself in this situation? $(\mathrm{y} / \mathrm{n})$

Probe: What was it about the situation that led you to think this way?

What did you experience in your body in response to this situation? Did you have any physical sensations? (If participant unsure say): Like sweating, shaking, or muscle tension? (y/n)

Probe: How intensely did you experience these sensations from 0 (hardly noticeable) to 10 (the most strongly I've ever felt this sensation)? $/ 10$

What did you do in response to this situation? That is, what actions did you take or not take?

Probe: How would you feel if you didn't do those things?

Probe: What was it about the situation that led you to do those things?

Are there any other ways that you responded to this uncertain situation that you can recall?

Different Responses to Uncertainty:

Do you ever react in different ways than what we just discussed when you are in uncertain situations? (y/n)

(If yes): Can you tell me about any different responses to uncertainty that you've had?

(If yes): What kind of situation(s) cause(d) you to react in this different way?

Positive Responses to Uncertainty: 
(If participant described uncertainty as only negative, say): Is uncertainty ever a good thing?

(If participant unsure say): Is uncertainty ever exciting? Or a chance to learn something new? $(\mathrm{y} / \mathrm{n})$

(If yes): Can you give me an example?

(If no): What do you think it is about uncertainty that make you feel like it is never a good thing?

What about your responses to uncertain situations that might be considered positive, or that are likely to turn out positively? For example, how do you react to not knowing what you will get as a gift?

(Other examples: how do you react to not knowing what you will do on a day off? Or if you know that you will be getting a raise but you do not know by how much?)

Clarifying whether responses are to uncertainty itself or responses are to possible negative outcomes:

(if participant reacts negatively to uncertain situations): Do you feel bothered by/anxious in (use participants own words) just about any uncertain situation, or are you only bothered by uncertain situations with a possible negative outcome?

-(if any uncertainty): What do you think it is about uncertainty in general that is so bothersome?

Negative Responses to Uncertainty (if not already discussed):

(If participant hasn't discussed their responses to uncertainty about negative outcomes prior to this point): What about your responses to uncertain situations that might be considered negative or are likely to turn out negatively. In other words, how do you react to not knowing whether something will turn out badly? For example, not knowing whether you will be fired from a job.

\section{Reasons underlying beliefs about uncertainty}

We've talked about how your react in uncertain situations, now I want to understand more about your attitudes toward uncertain situations and uncertainty in general.

How do you feel about uncertainty? That is, is uncertainty a good thing or a bad thing or is it neither?

Probe: What do you think it is about uncertainty or being uncertain that makes you feel that way?

Probe: Downward arrow answer if possible:

what would be so bad about that or what does that mean about you? 
Probe: (If participant describes uncertainty as sometimes good, sometimes bad, ask):

What is it that makes uncertainty good/neutral sometimes and bad other times?

Does begin uncertain ever mean anything about you personally? (y/n)

If participant says that they do not understand, say, "for example, some people might say that being uncertain means that they are a disorganized person, has being uncertain ever made you think anything personal like this about yourself?”

(If yes): What does being uncertain mean about you personally?

(If yes): What do you think it is about being uncertain that makes you feel like you are (insert response from above)?

(If no): What is it about being uncertain that makes you say that it is not personal?

Can you give me an example of a time when it felt unfair to be uncertain or unfair to not know things?

(If participant unsure say): Is it ever unfair not to have guarantees about something or to not know what will happen next?

Probe: What was it about that situation that seemed unfair?

Probe: What emotions or moods did you feel in that situation?

Probe: How did you behave or act in that situation?

Does it ever feel unfair when other people aren't uncertain but you are?

Can you think of some examples of when you felt like this?

\section{Potential Moderators of Responses to Uncertainty}

\section{Probability}

How do you feel in uncertain situations when you know that there's only a small chance of something bad happening?

Probe: Can you give me an example of a time where a small chance of something bad happening (bothered you/did not bother you)?

Do you react and worry the same way when of the chance of bad happening is big or small? 
(If yes): Why do you think that you still worry even when the chance of something bad happening is small?

\section{Cost}

How do you feel in uncertain situations where the consequences of something bad happening are minor?

(If participant unsure say): For example, you woke up late and are unsure whether you will be a few minutes late to meet a friend.

Probe: Can you give me an example of a time where minor consequences of being uncertain (bothered you/did not bother you)?

Do you feel the same way regardless of whether the consequences of something bad happening are minor or major?

(If yes):Why do you think that you still worry even when the consequences of something bad happening are minor?

\section{Unknown/Unknowable:}

Do you feel differently about uncertainty when others are also uncertain, compared to when others have more information than you have? For example, if others found out about the outcome of an interview before you did, compared to you and others all being uncertain about the outcome of an interview?

Why do you think that is?

\section{Controllability:}

How do you feel in uncertain situations that are more in your control (e.g., uncertainty about how you will do on an exam that you could study for, or uncertainty about whether you will be late) compared to uncertain situations that are less in your control (e.g., uncertainty about a loved one's health, or uncertainty about the weather)?

Why do you think that is?

\section{Domain:}

Do you feel differently about uncertainty in certain areas of your life? For example, is uncertainty in one area worse than uncertainty in other areas?

Why do you think that is? 


\section{Clarifying the Extent of Intolerance of Uncertainty}

Some people say that they would prefer a more negative outcome than an uncertain outcome. For example, if someone is fighting a lot with their romantic partner and feeling uncertain about how their romantic relationship will turn out, some people say that they would prefer that their romantic partner would just leave them now so that they will know with certainty what will happen, as opposed to remaining uncertain about the outcome. What do you think about this?

What is it about this that makes you prefer (certainty/uncertainty)?

(If prefer certainty): is the preference for uncertainty related to wanting to just feel bad now, as opposed to possibly unexpectedly feeling bad later?

If a person is allergic to something, they tend to have stronger responses than most people to even small amounts of the thing that they are allergic to. Some people have used the analogy "allergic to uncertainty" to describe how they react to and feel about uncertainty. What do you think about this idea that some people may be "allergic" to uncertainty?

How does this idea relate to you? 


\section{Behavioural Responses to Uncertainty (type, frequency):}

Now that you have told me about how you act in response to one (a few) uncertain situation(s), I'd like to spend some more time talking about things that you may or may not do in uncertain situations. I'm going to go through a list of different things that people may do when they are uncertain. First, I'd like you to just answer yes or no to let me know whether or not you have done these things when you are uncertain in the past month, and then I'd like you to estimate how many times in the past month you did each thing.

(Read prompt followed by behaviour in the $1^{\text {st }}$ column, for each behaviour in the list):

Prompt: During the past month, when you were uncertain, did you ever...

\begin{tabular}{|l|l|l|}
\hline Behaviour & Y/N & $\begin{array}{l}\text { Frequency } \\
\text { (how often) }\end{array}$ \\
\hline Ask for other people's opinion that what you did was okay? & & \\
\hline $\begin{array}{l}\text { Ask other people for reassurance about how something will turn out? } \\
\text { (If participant unclear on meaning of reassurance say: ask people to tell } \\
\text { you that it will be “okay” even if you have already received this } \\
\text { information in the past) }\end{array}$ & & \\
\hline $\begin{array}{l}\text { Seeking reassurance or opinions from others about how to make an } \\
\text { everyday decision? }\end{array}$ & & \\
\hline $\begin{array}{l}\text { Reassure yourself (in your head or aloud) that everything will be okay, } \\
\text { or that you did everything correctly? }\end{array}$ & & \\
\hline $\begin{array}{l}\text { Seek out } \text { a lot of information? (i.e., more information than most people } \\
\text { would seek out)? }\end{array}$ & & \\
\hline Repeatedly check things over (i.e., check things more than once)? & & \\
\hline $\begin{array}{l}\text { Spend more time than you think is actually necessary planning or } \\
\text { preparing for something (e.g., a routine meeting with your peers)? }\end{array}$ & & \\
\hline $\begin{array}{l}\text { Do things for other people that they could do for themselves (e.g., } \\
\text { make appointments for your partner)? }\end{array}$ & & \\
\hline $\begin{array}{l}\text { 'Overprotect' others (e.g., call your partner or grown child often to } \\
\text { check to see that they are 'okay')? }\end{array}$ & & \\
\hline $\begin{array}{l}\text { Not delegate tasks to others, and do everything (or most things) } \\
\text { yourself? }\end{array}$ & & \\
\hline $\begin{array}{l}\text { Make work-related or personal commitments that you really do not } \\
\text { want to make (e.g., taking on more work than you would like to)? }\end{array}$ & & \\
\hline Try to control everything (e.g., try to control what others do)? & & \\
\hline
\end{tabular}




\begin{tabular}{|l|l|l|}
\hline $\begin{array}{l}\text { Overestimate the chance that bad things will happen, and prepare for } \\
\text { the worst? }\end{array}$ & & \\
\hline $\begin{array}{l}\text { Rehearse what you will say or do in a situation (e.g., rehearse what you } \\
\text { will say when you meet a friend)? }\end{array}$ & & \\
\hline $\begin{array}{l}\text { Avoid doing things (e.g., avoid taking on a new role at work with } \\
\text { responsibilities that you are uncertain that you can handle)? }\end{array}$ & & \\
\hline $\begin{array}{l}\text { Not fully commit to things (e.g., projects or relationships with an } \\
\text { unclear outcome)? }\end{array}$ & & \\
\hline Procrastinate? (put off what one could do now to a later time)? & & \\
\hline Not take any action (i.e., do nothing)? & & \\
\hline Distract yourself by doing something else? & & \\
\hline Worry? & & \\
\hline Doubt or reconsider decisions that you have already made? & & \\
\hline Try to push away thoughts of the uncertain situation? & & \\
\hline $\begin{array}{l}\text { Try to replace negative thoughts with more neutral or positive thoughts } \\
\text { (e.g., replace thoughts of an event turning out badly with minor details } \\
\text { of the event)? }\end{array}$ & & \\
\hline Take medications or use other substances? & & \\
\hline uncertain about)? & & \\
\hline $\begin{array}{l}\text { Not act, even when a situation requires you to act quickly (e.g., not be } \\
\text { able to make up your mind before the deadline of an opportunity)? }\end{array}$ & & \\
\hline Make sure that you maintain a specific routine(s)? \\
uncertat try not to pay attention to an upcoming assignment that you are \\
\hline $\begin{array}{l}\text { Seek out more information about your own role in a particular } \\
\text { situation? }\end{array}$ & & \\
\hline Acknowledge and accept uncertainty? & & \\
\hline
\end{tabular}


Is there anything else that you can think of that you do when you are uncertain?

Now, I'd like to ask you some questions about the main behaviours that you do when you are uncertain, that is, (list most frequent beahviours).

\section{Cessation Factors:}

If you are doing $\mathrm{X}$, what causes you to stop doing it?

Function:

Now, I'd like to ask you about the function of the main behaviours that you use when you are uncertain, in other words, what you get out of doing each of these things. What leads you to do $\mathrm{X}$ ? (Does it Change something about the way you feel? Does it change something about the situation?)

\section{Impairment associated with behaviours:}

Of these behaviours that we discussed, do any cause problems for you?

(If yes): Which behaviours cause problems for you?

(If yes): In what ways does this behaviour/these behaviours cause problems for you?

Understanding type of trigger (more major vs minor sources of uncertainty):

It sounds like the main behaviours that you use when you are uncertain are $\mathrm{X}, \mathrm{Y}$, and $\mathrm{Z}$ (list participants' most frequently used behaviours). Can you tell me a bit about what kinds of uncertain situations lead you to engage in these behaviours?

Do everyday uncertain situations lead you to use these behaviours?

Can you give me some examples?

\section{Anger/Frustration:}

Has uncertainty ever made you feel annoyed, frustrated, or angry? (y / n)

(If participant has already mentioned this): I'd like to ask you more about when uncertainty makes you feel annoyed/frustrated/angry.

(If not yet discussed): Can you give me an example of a time where feeling uncertain or being in an uncertain situation made you feel angry?

(If already discussed): Can you give me any other examples of times when feeling uncertain made you feel angry? 
What was it about the situation that made you feel angry?

How do you express your anger in situations like these?

(If participant unsure): For example, do you hold in your anger inside in these situations, or do something to express that anger outwardly?

Questions specifically about hypotheses:

Pessimism as a reason for beliefs underlying uncertainty:

When you don't know how something will turn out, do you tend to think that it will turn out negatively?

Can you give me an example when you were pessimistic about how an uncertain situation would turn out?

Beliefs in personal ineffectiveness or inability to cope as a reason for beliefs underlying uncertainty:

Do you feel unsure about whether or not you will be able to cope with how something will turn out when you are uncertain?

\section{Conclusion}

Is there anything else that you think would be important for me to know about your experience with uncertainty that I haven't asked you about?

That concludes the interview portion of this study. Thank you for your time and for answering these personal questions. Do you have any questions for me before we move on to the next part of the study? 
Appendix C - Codebook for Interviews

\section{Study 1a: Attitudes Toward Uncertainty}

\begin{tabular}{|c|c|c|c|}
\hline \multicolumn{4}{|c|}{ Thoughts in a Typical Uncertain Situation over the Past Week } \\
\hline Code & Definition & $\begin{array}{l}\text { Coding Rules } \\
\text { (Code when a participant } \\
\text { describes...) }\end{array}$ & Examples \\
\hline Negative Outcomes & $\begin{array}{l}\text { Negative thoughts } \\
\text { about how a situation } \\
\text { will resolve }\end{array}$ & $\begin{array}{l}\text { 1) believing that the situation will } \\
\text { resolve negatively } \\
\text { 2) focusing on possible negative } \\
\text { outcomes } \\
\text { Note: Do not use when for passing } \\
\text { thoughts of negative outcomes that } \\
\text { they don't necessarily believe will } \\
\text { happen }\end{array}$ & "I just felt I was going to do bad." \\
\hline Positive Outcomes & $\begin{array}{l}\text { Positive thoughts } \\
\text { about how the } \\
\text { situation will resolve }\end{array}$ & $\begin{array}{l}\text { 1) trying to stay positive } \\
\text { 2) thoughts of positive outcomes } \\
\text { 3) a belief that things will resolve } \\
\text { positively }\end{array}$ & $\begin{array}{l}\text { "I thought well if they don't see it now and } \\
\text { they might see it in the morning and } \\
\text { maybe that won't be too late. I just tried to, } \\
\text { tried to think positive." }\end{array}$ \\
\hline Negative self-thoughts & $\begin{array}{l}\text { Negative thoughts } \\
\text { related to the self } \\
\text { when uncertain }\end{array}$ & $\begin{array}{l}\text { 1) doubting self or ability } \\
\text { 2) not knowing what to do } \\
\text { 3) self-criticism } \\
\text { 4) thinking one's thoughts are } \\
\text { "wrong” or "bad" }\end{array}$ & $\begin{array}{l}\text { "I felt deficient. I just couldn’t keep up } \\
\text { with basic things.” }\end{array}$ \\
\hline Positive self-thoughts & $\begin{array}{l}\text { Positive thoughts } \\
\text { related to the self } \\
\text { when uncertain }\end{array}$ & $\begin{array}{l}\text { 1) confidence in self } \\
\text { 2) feeling able to handle the situation } \\
\text { 3) any other positive self- } \\
\text { descriptions }\end{array}$ & $\begin{array}{l}\text { "I think I can handle it. I look on it as a } \\
\text { challenge" }\end{array}$ \\
\hline
\end{tabular}




\begin{tabular}{|c|c|c|c|}
\hline Desire for certainty & $\begin{array}{l}\text { Thoughts of wanting } \\
\text { the uncertainty to } \\
\text { resolve }\end{array}$ & $\begin{array}{l}\text { 1) wanting to get uncertainty over } \\
\text { with } \\
\text { 2) wanting or 'needing' to know }\end{array}$ & $\begin{array}{l}\text { "Ya, like I don’t want to be here, I just } \\
\text { want to get it over with, and I don’t want } \\
\text { to deal with it." }\end{array}$ \\
\hline Unfairness & $\begin{array}{l}\text { Thoughts that the } \\
\text { uncertainty is unfair or } \\
\text { something about the } \\
\text { situation is unfair }\end{array}$ & $\begin{array}{l}\text { 1) uncertainty as unfair } \\
\text { 2) thinking "why is this happening to } \\
\text { me” } \\
\text { 3) being thwarted from getting } \\
\text { information }\end{array}$ & $\begin{array}{l}\text { "Like what did I do wrong? I was so } \\
\text { careful about it. Like why was this } \\
\text { happening today when I have an audition } \\
\text { tomorrow?" }\end{array}$ \\
\hline Acceptance & $\begin{array}{l}\text { Thoughts related to } \\
\text { accepting the } \\
\text { uncertain situation }\end{array}$ & $\begin{array}{l}\text { 1) trying to make the most of things } \\
\text { 2) discussing acceptance of } \\
\text { uncertainty } \\
\text { 3) recognizing the inevitability of } \\
\text { being uncertain }\end{array}$ & $\begin{array}{l}\text { "It is part of life so you just kind of have to } \\
\text { deal with it" }\end{array}$ \\
\hline \multicolumn{4}{|c|}{ Reasons Uncertainty is Negative } \\
\hline $\begin{array}{l}\text { Because it interferes } \\
\text { with preparation and } \\
\text { control }\end{array}$ & $\begin{array}{l}\text { Refers to people not } \\
\text { liking uncertainty } \\
\text { because they feel } \\
\text { unable to adequately } \\
\text { plan, prepare, or be 'in } \\
\text { control' }\end{array}$ & $\begin{array}{l}\text { 1) uncertainty being negative } \\
\text { because of a need to plan } \\
\text { 2) uncertainty being negative } \\
\text { because of a need to prepare } \\
\text { 3) uncertainty being negative } \\
\text { because they are not in control }\end{array}$ & $\begin{array}{l}\text { "just anything that's the unknown is } \\
\text { definitely a little scary to me. Because I } \\
\text { am such a planner. Um to the point that I } \\
\text { plan you know where I wanna be } 5 \text { years, } \\
\text { from now } 10 \text { years from now... anything } \\
\text { unexpected is just kinda, definitely scares } \\
\text { me." } \\
\text { "Immediately what comes to my mind not } \\
\text { having control." }\end{array}$ \\
\hline $\begin{array}{l}\text { Because of the } \\
\text { feelings/responses it } \\
\text { causes me }\end{array}$ & $\begin{array}{l}\text { This refers to people } \\
\text { not liking uncertainty } \\
\text { because of how it } \\
\text { makes them feel; i.e., }\end{array}$ & $\begin{array}{l}\text { 1) uncertainty being negative } \\
\text { because of their response to it } \\
\text { (generally) }\end{array}$ & $\begin{array}{l}\text { "Because uncertainty is at the root of a lot } \\
\text { of my anxiety. So if there wasn’t such } \\
\text { thing as uncertainty, I wouldn’t have so }\end{array}$ \\
\hline
\end{tabular}




\begin{tabular}{|c|c|c|c|}
\hline & $\begin{array}{l}\text { because it causes } \\
\text { negative feelings, } \\
\text { thoughts or physical } \\
\text { responses. }\end{array}$ & $\begin{array}{l}\text { 2) uncertainty being negative } \\
\text { because of emotions, physical } \\
\text { responses, thoughts, or behaviours it } \\
\text { causes or leads to in themselves }\end{array}$ & $\begin{array}{l}\text { much anxiety.” } \\
\text { I. Um and what makes you hate } \\
\text { uncertainty? } \\
\text { P. The way it makes me feel I guess }\end{array}$ \\
\hline $\begin{array}{l}\text { Because I assume } \\
\text { worst case scenario }\end{array}$ & $\begin{array}{l}\text { This refers to people } \\
\text { saying that uncertainty } \\
\text { is bad because they } \\
\text { tend to think that the } \\
\text { worst is going to } \\
\text { happen }\end{array}$ & $\begin{array}{l}\text { 1) uncertainty being negative } \\
\text { because the person has a tendency to } \\
\text { assume the worst or a tendency to } \\
\text { think of negative outcomes } \\
\text { Do not code for assuming worst in } \\
\text { specific case; code refers to general } \\
\text { tendency }\end{array}$ & $\begin{array}{l}\text { "I tend to believe that most uncertain } \\
\text { situations will turn out negative" } \\
\text { "because I think worst case scenario, so } \\
\text { when you don't know the outcome, I am } \\
\text { someone who thinks like, if someone's } \\
\text { late, I'm like, oh, they got in a car } \\
\text { accident..." }\end{array}$ \\
\hline $\begin{array}{l}\text { Because it can resolve } \\
\text { negatively }\end{array}$ & $\begin{array}{l}\text { This refers to people } \\
\text { saying that uncertainty } \\
\text { is negative because it } \\
\text { could result in } \\
\text { negative outcomes at } \\
\text { times. }\end{array}$ & $\begin{array}{l}\text { 1) uncertainty being negative } \\
\text { because of the possibility of negative } \\
\text { outcomes (described generally) } \\
\text { 2) uncertainty being negative if } \\
\text { specific uncertain situations resolve } \\
\text { negatively (i.e., people give their } \\
\text { own examples of a situation that } \\
\text { could resolve negatively instead of } \\
\text { explaining this in general or vague } \\
\text { terms) } \\
\text { 3) code only if considering a } \\
\text { negative outcome when uncertain is } \\
\text { not described as a general tendency } \\
\text { (if so, code under assume worst case } \\
\text { scenario) } \\
\text { 4) do not code if participant } \\
\text { describes assuming worst case }\end{array}$ & $\begin{array}{l}\text { "I guess it would be the fear of something } \\
\text { bad happening, I guess that would exist" } \\
\text { "what's bad about that [uncertainty] is... } \\
\text { say what happens in the end is a negative } \\
\text { thing that you don't want" }\end{array}$ \\
\hline
\end{tabular}




\begin{tabular}{|c|c|c|c|}
\hline & & $\begin{array}{l}\text { scenario in general (then it can be } \\
\text { assumed that uncertainty is negative } \\
\text { because of negative outcomes in } \\
\text { general as opposed to in specific } \\
\text { situations) }\end{array}$ & \\
\hline $\begin{array}{l}\text { When negative } \\
\text { outcomes are likely or } \\
\text { severe }\end{array}$ & $\begin{array}{l}\text { This refers to people } \\
\text { saying that uncertainty } \\
\text { is negative when } \\
\text { negative outcomes are } \\
\text { likely to occur or are } \\
\text { very severe }\end{array}$ & $\begin{array}{l}\text { 1) uncertainty being negative in } \\
\text { situations wherein negative outcomes } \\
\text { are likely to occur } \\
\text { Or } \\
\text { 2) uncertainty being negative in } \\
\text { situations wherein negative outcomes } \\
\text { are serious }\end{array}$ & $\begin{array}{l}\text { "when the consequence is severe" } \\
\text { "if those [negative outcomes] are very } \\
\text { strong and very likely" }\end{array}$ \\
\hline \multicolumn{4}{|c|}{ Pessimism when uncertain } \\
\hline Code & Definition & $\begin{array}{l}\text { Coding Rules } \\
\text { (Code when a participant } \\
\text { describes...) }\end{array}$ & Examples \\
\hline Pessimistic & $\begin{array}{l}\text { Tendency to be } \\
\text { pessimistic about } \\
\text { outcome when } \\
\text { uncertain }\end{array}$ & $\begin{array}{l}\text { 1) General pessimism about how } \\
\text { things will resolve when they are } \\
\text { uncertain }\end{array}$ & "I always assume the negative." \\
\hline Optimistic & $\begin{array}{l}\text { Tendency to be } \\
\text { optimistic about } \\
\text { outcome when } \\
\text { uncertain }\end{array}$ & $\begin{array}{l}\text { 1) General optimism about how } \\
\text { things will resolve when they are } \\
\text { uncertain }\end{array}$ & "No I am an optimistic guy” \\
\hline Realist & $\begin{array}{l}\text { No tendency toward } \\
\text { assuming either } \\
\text { positive or negative } \\
\text { outcomes when } \\
\text { uncertain }\end{array}$ & $\begin{array}{l}\text { 1) No strong bias toward optimism or } \\
\text { pessimism when uncertain } \\
\text { 2) Being neutral when uncertain } \\
\text { 3) Not having fixed assumptions } \\
\text { about how uncertainty will resolve }\end{array}$ & $\begin{array}{l}\text { "Yes and No. Yes because I always } \\
\text { prepare for the worst.... I never say it's } \\
\text { going to be good so who cares, I always } \\
\text { prepare for the worst, but hope for the } \\
\text { best." }\end{array}$ \\
\hline
\end{tabular}




\begin{tabular}{|c|c|c|c|}
\hline \multicolumn{4}{|c|}{ Inefficacy when uncertain } \\
\hline Can’t cope & $\begin{array}{l}\text { Beliefs in inability to } \\
\text { cope when uncertain }\end{array}$ & $\begin{array}{l}\text { 1) General tendency to doubt ability } \\
\text { to cope when uncertain }\end{array}$ & $\begin{array}{l}\text { "Even if it's a physical response or a } \\
\text { mental response.... I don't know how I'll } \\
\text { react. For example if I have to go through } \\
\text { chemo... first of all I don't know what the } \\
\text { outcome to chemo will be, and second of } \\
\text { all, I don't know how I'm going to feeling } \\
\text { when doing it, or living when doing it.” }\end{array}$ \\
\hline Can cope & $\begin{array}{l}\text { Beliefs in ability to } \\
\text { cope when uncertain }\end{array}$ & $\begin{array}{l}\text { 1) General tendency to cope when } \\
\text { uncertain } \\
\text { 2) Disagreement with question about } \\
\text { a tendency to assume one cannot } \\
\text { cope when uncertain }\end{array}$ & $\begin{array}{l}\text { "No usually I think I'll be able to deal with } \\
\text { whatever comes. In one way or another." }\end{array}$ \\
\hline Depends on situation & $\begin{array}{l}\text { Beliefs in ability to } \\
\text { cope under some } \\
\text { circumstances when } \\
\text { uncertain }\end{array}$ & $\begin{array}{l}\text { 1) At times doubt ability to cope, } \\
\text { although general tendency is not } \\
\text { clear }\end{array}$ & $\begin{array}{l}\text { "Sometimes. If it does end up negatively I } \\
\text { don't know how I am going to deal with } \\
\text { that situation. And that can worry me. I } \\
\text { have outburst in the street before and when } \\
\text { I can't cope I will start crying and stuff. I } \\
\text { am always afraid something like that will } \\
\text { happen.” }\end{array}$ \\
\hline \multicolumn{4}{|c|}{ Personal Meanings of Being Uncertain } \\
\hline I'm abnormal & $\begin{array}{l}\text { Being uncertain } \\
\text { means I am } \\
\text { abnormal in some } \\
\text { way }\end{array}$ & $\begin{array}{l}\text { 1) response reflects idea that one is } \\
\text { uptight, anxious, not normal, more } \\
\text { worried than others, reacting } \\
\text { differently than others, more worried } \\
\text { than they ought to be, bad, or flawed } \\
\text { when uncertain }\end{array}$ & $\begin{array}{l}\text { "Uh I tend to think that I overreact in } \\
\text { uncertain situations more so then normal } \\
\text { people" }\end{array}$ \\
\hline $\begin{array}{l}\text { I'm } \\
\text { incapable/incompetent }\end{array}$ & $\begin{array}{l}\text { Being uncertain } \\
\text { means I am }\end{array}$ & $\begin{array}{l}\text { 1) response reflects idea that one is } \\
\text { incapable, useless, less smart, }\end{array}$ & “I don’t feel capable" \\
\hline
\end{tabular}




\begin{tabular}{|c|c|c|c|}
\hline & $\begin{array}{l}\text { incapable or } \\
\text { incompetent }\end{array}$ & $\begin{array}{l}\text { inadequate or incompetent when } \\
\text { uncertain } \\
\text { Exception: if person says that they've } \\
\text { felt unconfident when uncertain in } \\
\text { the rare case (i.e., very infrequent), } \\
\text { do not code here }\end{array}$ & \\
\hline I'm unprepared & $\begin{array}{l}\text { Being uncertain } \\
\text { means I’m not } \\
\text { prepared }\end{array}$ & $\begin{array}{l}\text { 1) response reflects idea that one is: } \\
\text { ill-prepared, forgot something, didn't } \\
\text { do enough, missed something, should } \\
\text { have done more, not on top of things, } \\
\text { should have known better when } \\
\text { uncertain }\end{array}$ & $\begin{array}{l}\text { “well I kinda see it as you're not prepared, } \\
\text { you're not um you're not on top of your } \\
\text { stuff” }\end{array}$ \\
\hline $\begin{array}{l}\text { Positive personal } \\
\text { meaning }\end{array}$ & $\begin{array}{l}\text { Being uncertain } \\
\text { means something } \\
\text { positive about } \\
\text { person }\end{array}$ & $\begin{array}{l}\text { 1) noticing some positive attribute } \\
\text { such as spontaneity or confidence } \\
\text { when uncertain }\end{array}$ & "It makes me feel spontaneous" \\
\hline No personal meaning & $\begin{array}{l}\text { Being uncertain } \\
\text { doesn't have any } \\
\text { personal meaning; } \\
\text { uncertainty is not } \\
\text { personal }\end{array}$ & $\begin{array}{l}\text { 1) response reflects the idea that } \\
\text { being uncertain isn't personal, or } \\
\text { doesn't mean anything about them }\end{array}$ & $\begin{array}{l}\text { "Uncertainty, how does it make me feel } \\
\text { about myself? Do you know, I don’t think } \\
\text { I think about myself when I'm uncertain” }\end{array}$ \\
\hline \multicolumn{4}{|c|}{ Reasons uncertainty is unfair } \\
\hline $\begin{array}{l}\text { Others are better at } \\
\text { coping with uncertainty } \\
\text { than me }\end{array}$ & $\begin{array}{l}\text { Uncertainty is unfair } \\
\text { because person feels } \\
\text { they don't cope well } \\
\text { with uncertainty and } \\
\text { other people do }\end{array}$ & $\begin{array}{l}\text { Uncertainty as unfair when others } \\
\text { 1) did not have negative responses to } \\
\text { uncertainty, such as worry, when the } \\
\text { participant did } \\
\text { 2) others seemed sure of themselves } \\
\text { or confident when uncertain and the } \\
\text { person had difficulty with this }\end{array}$ & $\begin{array}{l}\text { "I wish I didn’t have so much to worry } \\
\text { about while as he seems so calm, cool, and } \\
\text { collected" }\end{array}$ \\
\hline
\end{tabular}




\begin{tabular}{|c|c|c|c|}
\hline $\begin{array}{l}\text { I don't deserve to be } \\
\text { uncertain }\end{array}$ & $\begin{array}{l}\text { Uncertainty is unfair } \\
\text { because I didn't do } \\
\text { anything to induce it }\end{array}$ & $\begin{array}{l}\text { Uncertainty as unfair due to it being } \\
\text { undeserved } \\
\text { 1) it could have happened to anyone, } \\
\text { they were 'randomly selected' } \\
\text { 2) uncertainty not their fault } \\
\text { 3) their behaviours did not warrant } \\
\text { feeling uncertain }\end{array}$ & $\begin{array}{l}\text { “We felt attacked and didn’t feel like we } \\
\text { deserved it especially since her complaints } \\
\text { about us were just things that people do } \\
\text { like cooking, laughing and whatnot. So } \\
\text { yeah we felt unjustly attacked” [about } \\
\text { uncertainty re housing because landlord } \\
\text { was asking family to leave] }\end{array}$ \\
\hline $\begin{array}{l}\text { It's others' fault I'm } \\
\text { uncertain }\end{array}$ & $\begin{array}{l}\text { Uncertainty is unfair } \\
\text { because it is 'caused' } \\
\text { by another person or } \\
\text { entity }\end{array}$ & $\begin{array}{l}\text { Uncertainty as unfair as it was/is due } \\
\text { to another person or entity } \\
\text { 1) themes of withholding information } \\
\text { 2) actions of others creating } \\
\text { uncertain situation }\end{array}$ & $\begin{array}{l}\text { "it felt unfair...probably all of two minutes } \\
\text { [for them to call me] and I'm sitting there } \\
\text { half an hour and not knowing when they're } \\
\text { going to call me” }\end{array}$ \\
\hline $\begin{array}{l}\text { The outcome of an } \\
\text { uncertain situation is } \\
\text { very important or serious }\end{array}$ & $\begin{array}{l}\text { Uncertainty is unfair } \\
\text { because of the } \\
\text { severity of the } \\
\text { implications of a } \\
\text { possible negative } \\
\text { outcome }\end{array}$ & $\begin{array}{l}\text { Uncertainty as unfair due to the } \\
\text { magnitude of the circumstance that } \\
\text { the unknown is about }\end{array}$ & $\begin{array}{l}\text { "When he's gone in for surgery, it's not } \\
\text { fair that I don’t know what's going to } \\
\text { happen, because it’s such a big thing....it } \\
\text { has such big implications.” }\end{array}$ \\
\hline \multicolumn{4}{|c|}{ Potential moderators of responses to uncertainty } \\
\hline $\begin{array}{l}\text { Probability - changes } \\
\text { response }\end{array}$ & $\begin{array}{l}\text { Likelihood of a } \\
\text { possible negative } \\
\text { outcome changes } \\
\text { person's response }\end{array}$ & $\begin{array}{l}\text { 1) probability clearly moderates } \\
\text { response } \\
\text { 2) probability somewhat moderates } \\
\text { response (i.e., sometimes, to an } \\
\text { extent, a little) }\end{array}$ & $\begin{array}{l}\text { Because the outcome is greater, or the } \\
\text { chances are higher, yeah, oh yeah } \\
\text { definitely feel worse }\end{array}$ \\
\hline $\begin{array}{l}\text { Probability - does not } \\
\text { change response }\end{array}$ & $\begin{array}{l}\text { Likelihood of a } \\
\text { possible negative }\end{array}$ & $\begin{array}{l}\text { 1) probability does not change } \\
\text { response }\end{array}$ & $\begin{array}{l}\text { I still see it as, bad is bad... To me a little is } \\
\text { just as important. Especially if I can do }\end{array}$ \\
\hline
\end{tabular}




\begin{tabular}{|c|c|c|c|}
\hline & $\begin{array}{l}\text { outcome DOES } \\
\text { NOT change } \\
\text { person's response }\end{array}$ & & $\begin{array}{l}\text { something to possibly prevent it, it will. } \\
\text { Like this meeting I had the other day, like I } \\
\text { pretty much I knew I had the volunteer } \\
\text { position but regardless I over prepared }\end{array}$ \\
\hline $\begin{array}{l}\text { Cost - changes } \\
\text { response }\end{array}$ & $\begin{array}{l}\text { Severity/magnitude/ } \\
\text { cost of a possible } \\
\text { negative outcome } \\
\text { changes person's } \\
\text { response }\end{array}$ & $\begin{array}{l}\text { 1) severity of outcome clearly } \\
\text { moderates response } \\
\text { 2) severity of outcome somewhat } \\
\text { moderates response (i.e., sometimes, } \\
\text { to an extent, a little) }\end{array}$ & $\begin{array}{l}\text { Yes. I would be more definitely more } \\
\text { anxious about being late about the job just } \\
\text { because I would expect a friend to } \\
\text { understand. I would have a good reason } \\
\text { and I would explain to them. But at a job } \\
\text { interview you are showing that you are not } \\
\text { punctual and the consequences are much } \\
\text { more serious. }\end{array}$ \\
\hline $\begin{array}{l}\text { Cost - does not change } \\
\text { response }\end{array}$ & $\begin{array}{l}\text { Severity/magnitude/ } \\
\text { cost of a possible } \\
\text { negative outcome } \\
\text { DOES NOT change } \\
\text { person's response to } \\
\text { uncertainty }\end{array}$ & 1) severity does not change response & $\begin{array}{l}\text { its negative whether the cost is little or } \\
\text { high like its still on me and my } \\
\text { responsibility to prevent that from } \\
\text { happening. }\end{array}$ \\
\hline $\begin{array}{l}\text { Others also being } \\
\text { uncertain - changes } \\
\text { response }\end{array}$ & $\begin{array}{l}\text { Others being } \\
\text { certain/having } \\
\text { knowledge whereas } \\
\text { participant is } \\
\text { uncertain changes } \\
\text { person's response to } \\
\text { uncertainty } \\
\text { *Code doesn't start } \\
\text { until IS09 }\end{array}$ & $\begin{array}{l}\text { 1) others certain of an outcome while } \\
\text { participant in the dark (uncertain) } \\
\text { clearly moderates response } \\
\text { 2) "somewhat moderates response } \\
\text { (i.e., sometimes, to an extent, a little) }\end{array}$ & $\begin{array}{l}\text { Yeah. I want to know everything. Why } \\
\text { don’t I know? Why do they know? Yes [it } \\
\text { changes my response]. Oh yeah. }\end{array}$ \\
\hline
\end{tabular}




\begin{tabular}{|c|c|c|c|}
\hline $\begin{array}{l}\text { Others also being } \\
\text { uncertain - does not } \\
\text { change response }\end{array}$ & $\begin{array}{l}\text { Others being } \\
\text { certain/having } \\
\text { knowledge whereas } \\
\text { participant is } \\
\text { uncertain DOES } \\
\text { NOT change } \\
\text { person's response to } \\
\text { uncertainty }\end{array}$ & $\begin{array}{l}\text { 1) others being certain does not } \\
\text { change response }\end{array}$ & $\begin{array}{l}\text { So I was like you know yours and Ill get to } \\
\text { know mine. That it was ok }\end{array}$ \\
\hline $\begin{array}{l}\text { Controllability - } \\
\text { changes response }\end{array}$ & $\begin{array}{l}\text { Degree of control } \\
\text { person has over } \\
\text { uncertain situation } \\
\text { changes person's } \\
\text { response to } \\
\text { uncertainty }\end{array}$ & $\begin{array}{l}\text { 1) degree of control person has } \\
\text { clearly moderates response } \\
\text { 2) degree of control person has } \\
\text { somewhat moderates response (i.e., } \\
\text { sometimes, to an extent, a little) }\end{array}$ & $\begin{array}{l}\text { I'm more bothered by things I don't have } \\
\text { control over, if cause if I have control over } \\
\text { it I'll utilize the control and like do what I } \\
\text { have to do to make it so. The idea of my } \\
\text { nervousness is at the minimal. But like if I } \\
\text { don't have a lot of control over it, if say I } \\
\text { am waiting for like a response from an } \\
\text { employer, like I can't really control I just } \\
\text { have to wait, like then I start getting really } \\
\text { nervous. }\end{array}$ \\
\hline $\begin{array}{l}\text { Controllability - } \\
\text { does not change } \\
\text { response }\end{array}$ & $\begin{array}{l}\text { Degree of control } \\
\text { person has over } \\
\text { uncertain situation } \\
\text { does not change } \\
\text { person's response to } \\
\text { uncertainty }\end{array}$ & $\begin{array}{l}\text { 1) degree of control person has does } \\
\text { not change response }\end{array}$ & $\begin{array}{l}\text { I: And does not having control over } \\
\text { uncertainty like there's nothing you can } \\
\text { about it... } \\
\text { P: I can't do anything for that... } \\
\text { I: Exactly, does that make it more } \\
\text { bothersome? } \\
\text { P: No because there's nothing I can do }\end{array}$ \\
\hline $\begin{array}{l}\text { Domain of life - } \\
\text { changes response }\end{array}$ & $\begin{array}{l}\text { The area of life } \\
\text { where the person } \\
\text { feels uncertain (e.g., } \\
\text { finances, family, }\end{array}$ & $\begin{array}{l}\text { 1) area of life where person feels } \\
\text { uncertain clearly moderates response } \\
\text { 2) area of life where person feels } \\
\text { uncertain somewhat moderates }\end{array}$ & $\begin{array}{l}\text { I think the only thing that I might be } \\
\text { dwelling on in terms of uncertainty is } \\
\text { finances }\end{array}$ \\
\hline
\end{tabular}




\begin{tabular}{|c|c|c|c|}
\hline & $\begin{array}{l}\text { work) changes } \\
\text { person's response to } \\
\text { uncertainty }\end{array}$ & $\begin{array}{l}\text { response (i.e., sometimes, to an } \\
\text { extent, a little) }\end{array}$ & \\
\hline $\begin{array}{l}\text { Domain of life - does } \\
\text { not change response }\end{array}$ & $\begin{array}{l}\text { The area of life } \\
\text { where the person } \\
\text { feels uncertain (e.g., } \\
\text { finances, family, } \\
\text { work) does not } \\
\text { change person's } \\
\text { response to } \\
\text { uncertainty }\end{array}$ & $\begin{array}{l}\text { 1) area of life where person feels } \\
\text { uncertain does not change response } \\
\text { (i.e., all areas life where person is } \\
\text { uncertain elicit same response) }\end{array}$ & $\begin{array}{l}\text { Umm no because you know have to keep } \\
\text { everything balanced right. I want to make } \\
\text { sure this is fine, this is fine, tick tick tick. } \\
\text { So I try to keep everything like stable and } \\
\text { balanced. So everything matters right, } \\
\text { people matter, money matters everything } \\
\text { matters. }\end{array}$ \\
\hline \multicolumn{4}{|c|}{ Bothered by uncertainty itself or the possibility of negative outcomes? } \\
\hline Uncertainty itself & $\begin{array}{l}\text { Uncertainty is bad in } \\
\text { and of itself. It is not } \\
\text { necessarily about the } \\
\text { possibility of } \\
\text { negative outcomes, it } \\
\text { is also that not } \\
\text { knowing is } \\
\text { uncomfortable. }\end{array}$ & $\begin{array}{l}\text { 1) having an aversion to not } \\
\text { knowing; really not liking not } \\
\text { knowing itself } \\
\text { 2) when person says it is feeling } \\
\text { uncertain that bothers them }\end{array}$ & $\begin{array}{l}\text { 'Like, the uncertainty of not knowing } \\
\text { makes me uncomfortable; I need to know } \\
\text { what's going on, and, even if its } \\
\text { unpredictable uncertainty, I want it to be } \\
\text { able to associate it with something, or } \\
\text { hopefully think about what possibilities are } \\
\text { related to this and how to deal with it; I } \\
\text { don't want to be surprised” }\end{array}$ \\
\hline $\begin{array}{l}\text { Possible negative } \\
\text { outcomes }\end{array}$ & $\begin{array}{l}\text { Uncertainty is } \\
\text { bothersome/upsettin } \\
\text { g when there is the } \\
\text { potential for possible } \\
\text { negative outcomes. } \\
\text { So uncertainty is bad } \\
\text { because it means that } \\
\text { things might turn out } \\
\text { badly. }\end{array}$ & $\begin{array}{l}\text { 1) being bothered by possible } \\
\text { negative outcomes/things turning out } \\
\text { badly AND says they are not } \\
\text { bothered by not knowing itself/the } \\
\text { feeling of uncertainty }\end{array}$ & $\begin{array}{l}\text { "I think that what's bothersome is a } \\
\text { possibility of negative outcomes. I don’t } \\
\text { think not knowing on its own is a bad } \\
\text { thing." }\end{array}$ \\
\hline
\end{tabular}




\begin{tabular}{|c|c|c|c|}
\hline Both & $\begin{array}{l}\text { Person is bothered } \\
\text { by both uncertainty } \\
\text { itself and uncertainty } \\
\text { about possible } \\
\text { negative outcomes }\end{array}$ & $\begin{array}{l}\text { 1) being bothered by possible } \\
\text { negative outcomes/things turning out } \\
\text { badly AND says they are ALSO } \\
\text { bothered by not knowing itself/the } \\
\text { feeling of uncertainty }\end{array}$ & $\begin{array}{l}\text { "I guess I'm bothered by not knowing } \\
\text { more often than not, and bothered if there } \\
\text { is a negative consequence that might } \\
\text { happen." }\end{array}$ \\
\hline \multicolumn{4}{|c|}{ When is uncertainty positive? } \\
\hline Excitement & $\begin{array}{l}\text { Uncertainty is good } \\
\text { because it is fun and } \\
\text { exciting }\end{array}$ & $\begin{array}{l}\text { 1) uncertainty being good because it } \\
\text { is fun or exciting, } \\
\text { 2) uncertainty keeping things from } \\
\text { being boring, or keeping things fresh } \\
\text { and new }\end{array}$ & $\begin{array}{l}\text { "It is exciting. What are we going to do } \\
\text { now? What ideas are we going to come up } \\
\text { with next? It is just crazy business so I } \\
\text { guess uncertainty is our lifestyle." }\end{array}$ \\
\hline Positive outcomes & $\begin{array}{l}\text { Uncertainty is good } \\
\text { because it could } \\
\text { mean positive } \\
\text { outcomes }\end{array}$ & $\begin{array}{l}\text { 1) uncertainty is good because it } \\
\text { could result in positive outcomes } \\
\text { 2) people say that uncertainty is good } \\
\text { when positive outcomes are likely or } \\
\text { they are confident about positive } \\
\text { outcomes }\end{array}$ & $\begin{array}{l}\text { "Maybe it works out for the best... we } \\
\text { don’t always know" } \\
\text { "it is good when it is, well when it has a } \\
\text { positive outcome obviously" }\end{array}$ \\
\hline $\begin{array}{l}\text { Minimal negative } \\
\text { outcomes }\end{array}$ & $\begin{array}{l}\text { Uncertainty is good } \\
\text { when there are no } \\
\text { negative outcomes } \\
\text { or only minor } \\
\text { negative outcomes }\end{array}$ & $\begin{array}{l}\text { uncertainty is okay/good in cases } \\
\text { where there are no negative } \\
\text { outcomes or when there are very } \\
\text { minor negative outcomes }\end{array}$ & $\begin{array}{l}\text { "if when it doesn’t work out it is not a big } \\
\text { deal” } \\
\text { "even if she had a girl I would have been } \\
\text { happy" }\end{array}$ \\
\hline Personal growth & $\begin{array}{l}\text { Uncertainty can lead } \\
\text { to self-improvement } \\
\text { or self-development. }\end{array}$ & $\begin{array}{l}\text { 1) personal growth/self- } \\
\text { improvement/self-development } \\
\text { through uncertainty } \\
\text { 2) uncertainty being good because it } \\
\text { is a personal challenge } \\
\text { 3) uncertainty as a chance to learn } \\
\text { something about themselves }\end{array}$ & $\begin{array}{l}\text { "Good in that it causes you to adopt and } \\
\text { grow because you have to adjust what you } \\
\text { are used and overcome any barriers that } \\
\text { are thrown in front of you." }\end{array}$ \\
\hline
\end{tabular}




\begin{tabular}{|c|c|c|c|}
\hline Learning & $\begin{array}{l}\text { Uncertainty can lead } \\
\text { to new learning, or } \\
\text { that being uncertain } \\
\text { is an opportunity to } \\
\text { learn something new }\end{array}$ & $\begin{array}{l}\text { 1) uncertainty as a chance to } \\
\text { learn/discover something } \\
\text { 2) uncertainty as a learning challenge }\end{array}$ & $\begin{array}{l}\text { "I can find uncertainty to be good at times } \\
\text { because I feel it promotes learning" }\end{array}$ \\
\hline Feeling free & $\begin{array}{l}\text { Uncertainty means } \\
\text { more freedom and } \\
\text { flexibility }\end{array}$ & $\begin{array}{l}\text { uncertainty as allowing for a } \\
\text { freedom/lack of structure/lack of } \\
\text { schedule/flexibility that is desirable }\end{array}$ & $\begin{array}{l}\text { "I guess when you are going out. You } \\
\text { don't really want to have a plan or } \\
\text { schedule at the time because it is not fun } \\
\text { just to like go somewhere and make sure } \\
\text { you have everything planned out, it is fun } \\
\text { to just go with the flow basically." }\end{array}$ \\
\hline \multicolumn{4}{|c|}{ Confusion at being asked about positive uncertainty } \\
\hline Yes & $\begin{array}{l}\text { Yes, person shows } \\
\text { confusion at being } \\
\text { asked about positive } \\
\text { uncertainty }\end{array}$ & $\begin{array}{l}\text { 1) asks for clarification about the } \\
\text { question or what positive uncertainty } \\
\text { is } \\
\text { 2) does not understand question } \\
\text { initally }\end{array}$ & $\begin{array}{l}\text { “Um but you don’t know it’s positive?” } \\
\text { “Um, what would be an example?” }\end{array}$ \\
\hline No & $\begin{array}{l}\text { No, person does not } \\
\text { show signs of } \\
\text { confusion at being } \\
\text { asked about positive } \\
\text { uncertainty }\end{array}$ & $\begin{array}{l}\text { 1) responds immediately to question } \\
\text { about positive uncertainty/clear } \\
\text { understanding of question apparent } \\
\text { 2) thinks of an example without } \\
\text { asking for clarification } \\
\text { 3) begins talking about positive } \\
\text { uncertainty spontaneously during } \\
\text { interview and provides examples } \\
\text { (without having to be asked question) }\end{array}$ & $\begin{array}{l}\text { 'Sure, sure. You don't want everything to } \\
\text { you know, to... Ya, you don’t want to be } \\
\text { sure of everything. Then life’s too easy. } \\
\text { Uncertainty is good. It's good. Yes.” }\end{array}$ \\
\hline
\end{tabular}




\begin{tabular}{|c|c|c|c|}
\hline \multicolumn{4}{|c|}{ How do you react to uncertainty about positive outcomes (i.e., uncertainty about receiving a gift?) } \\
\hline Positive emotions & $\begin{array}{l}\text { Positive emotions in } \\
\text { response to } \\
\text { uncertainty about } \\
\text { positive events }\end{array}$ & $\begin{array}{l}\text { 1) any positive emotion in response } \\
\text { to uncertainty } \\
\text { 2) a lack of or absence of negative } \\
\text { emotion in response to uncertainty } \\
\text { (for this, they have to articulate, e.g., } \\
\text { "I didn't feel bad, I was not } \\
\text { bothered”) } \\
\text { Do not code: } \\
\text { 1) person says its better than negative } \\
\text { uncertainty but still no positive } \\
\text { emotion }\end{array}$ & $\begin{array}{l}\text { "But obviously it will put me in a good } \\
\text { mood, and my expectations will be } \\
\text { positive." } \\
\text { "I would feel excited." }\end{array}$ \\
\hline Negative emotions & $\begin{array}{l}\text { Negative emotions } \\
\text { in response to } \\
\text { uncertainty about } \\
\text { positive events }\end{array}$ & $\begin{array}{l}\text { 1) describes any negative emotion in } \\
\text { response to positive uncertainty } \\
\text { 2) says that the positive uncertain } \\
\text { situation would be very hard for } \\
\text { them. }\end{array}$ & $\begin{array}{l}\text { "Umm...I do get nervous about receiving } \\
\text { gifts, except from like, my parents, but, } \\
\text { because I get nervous of how I will react." }\end{array}$ \\
\hline $\begin{array}{l}\text { Negative } \\
\text { expectancies/beliefs }\end{array}$ & $\begin{array}{l}\text { Negative } \\
\text { expectations or } \\
\text { beliefs about how } \\
\text { the uncertainty about } \\
\text { positive events will } \\
\text { resolve }\end{array}$ & $\begin{array}{l}\text { 1) describes how the situation could } \\
\text { resolve negatively } \\
\text { 2) describe a focus on possible } \\
\text { negative outcomes }\end{array}$ & $\begin{array}{l}\text { "Its a fear that I wont like it, but not } \\
\text { because of the actual gift itself, its that I } \\
\text { wont know how to react" } \\
\text { "I, I but it generally I tend to believe that } \\
\text { most uncertain situations will turn out } \\
\text { negative" }\end{array}$ \\
\hline
\end{tabular}




\begin{tabular}{|l|l|l|l|}
\hline \multicolumn{2}{|l|}{ Do you prefer a certain negative outcome to being uncertain? } \\
\hline $\begin{array}{l}\text { Certain negative } \\
\text { outcome }\end{array}$ & $\begin{array}{l}\text { Preference for a } \\
\text { definite negative } \\
\text { outcome over being } \\
\text { uncertain }\end{array}$ & $\begin{array}{l}\text { Prefer to know even if it is a negative } \\
\text { outcome (even if in some situations } \\
\text { would prefer to sit with uncertainty, } \\
\text { generally they prefer certainty) }\end{array}$ & $\begin{array}{l}\text { "I'd almost always rather just know. } \\
\text { Because then I could prepare for it." }\end{array}$ \\
\hline Being uncertain & $\begin{array}{l}\text { Preference for being } \\
\text { uncertain over a } \\
\text { definite negative } \\
\text { outcome }\end{array}$ & $\begin{array}{l}\text { Clearly prefer to sit with uncertainty } \\
\text { as opposed to taking a certain } \\
\text { negative outcome }\end{array}$ & $\begin{array}{l}\text { "Yeah the uncertain one” } \\
\text { "Id still look forward to it, like Im ok with } \\
\text { uncertainty, I would be prepared for both } \\
\text { but I wouldn't hope for the negative } \\
\text { outcome” }\end{array}$ \\
\hline It depends/unclear & $\begin{array}{l}\text { Response does not } \\
\text { show clear } \\
\text { preference for a } \\
\text { certain negative } \\
\text { outcome versus } \\
\text { being uncertain; or } \\
\text { preference is } \\
\text { domain-specific }\end{array}$ & $\begin{array}{l}\text { 1) an unclear response } \\
\text { 2) response that is consistent with } \\
\text { both codes for this question } \\
\text { 3) response varies by situation } \\
\text { 4) person cannot discern a preference }\end{array}$ & $\begin{array}{l}\text { "So I feel like I have more control over the } \\
\text { outcome, by even though its an uncertain } \\
\text { situation I have time where I guess the } \\
\text { ability to control how I prepare for it, than } \\
\text { the range is ok. In other situations where } \\
\text { just kind of thrown into me, or thrown into } \\
\text { it that set certain negative outcome might } \\
\text { be more, what I would prefer." }\end{array}$ \\
\hline
\end{tabular}




\begin{tabular}{|c|c|c|c|}
\hline \multicolumn{4}{|c|}{ Do you feel allergic to uncertainty? } \\
\hline Yes & $\begin{array}{l}\text { Yes I'm allergic to } \\
\text { uncertainty }\end{array}$ & $\begin{array}{l}\text { 1) the allergy analogy applies to } \\
\text { them } \\
\text { 2) agrees that they feel allergic to } \\
\text { uncertainty }\end{array}$ & $\begin{array}{l}\text { "Um so yeah for sure" } \\
\text { "I think thats like, kind of accurate to me” }\end{array}$ \\
\hline No & $\begin{array}{l}\text { No I'm not allergic } \\
\text { to uncertainty }\end{array}$ & $\begin{array}{l}\text { 1) the allergy analogy does not apply } \\
\text { to them } \\
\text { 2) disagrees that they feel allergic to } \\
\text { uncertainty }\end{array}$ & $\begin{array}{l}\text { "No. Definitely not, a little bit of } \\
\text { uncertainty, no it is part of life. I accept it } \\
\text { and it won't bother me that much so I } \\
\text { don't think it is the same response at all for } \\
\text { me in the strength of the response to a little } \\
\text { bit." }\end{array}$ \\
\hline It depends/unclear & $\begin{array}{l}\text { Unclear if I'm } \\
\text { allergic to } \\
\text { uncertainty, or } \\
\text { allergy depends on } \\
\text { the situation }\end{array}$ & $\begin{array}{l}\text { 1) unclear whether the analogy } \\
\text { applies to them } \\
\text { 2) agrees and disagrees with analogy } \\
\text { 3) allergy depends on situation/is } \\
\text { domain-specific }\end{array}$ & $\begin{array}{l}\text { "On a personal level I'm allergic to } \\
\text { uncertainty... and I'm very tolerant of it on } \\
\text { an impersonal level. Like if I'm uncertain } \\
\text { if my partner is cheating on me... I want to } \\
\text { know and ill work until I know.” }\end{array}$ \\
\hline
\end{tabular}




\section{Study 1b: Behavioural Responses to Uncertainty}

\begin{tabular}{|c|c|c|c|}
\hline Code & Definition & $\begin{array}{l}\text { Coding Rules } \\
\text { (Code when a participant } \\
\text { describes...) }\end{array}$ & Examples \\
\hline Worry & $\begin{array}{l}\text { Repetitive or } \\
\text { excessive thought } \\
\text { about how } \\
\text { something might } \\
\text { resolve in the future }\end{array}$ & $\begin{array}{l}\text { 1) being worried or worrying directly } \\
\text { 2) thinking through all possible } \\
\text { scenarios; 'over-thinking'; or } \\
\text { repeatedly thinking about a } \\
\text { situation/outcome/decision/event }\end{array}$ & $\begin{array}{l}\text { "but it was a lot of senseless worry" } \\
\text { "I thought about it and thought about it" }\end{array}$ \\
\hline Avoid & $\begin{array}{l}\text { Staying away from } \\
\text { situations with an } \\
\text { uncertain outcome }\end{array}$ & 1) avoiding uncertainty as defined & “I’ll hide away from it [uncertainty]" \\
\hline Distract & $\begin{array}{l}\text { Distracting oneself } \\
\text { from an uncertain } \\
\text { situation by doing } \\
\text { something else }\end{array}$ & 1) distracting self when uncertain & $\begin{array}{l}\text { "Distract myself with like going out or } \\
\text { calling friends" }\end{array}$ \\
\hline Do nothing & $\begin{array}{l}\text { Ceasing action when } \\
\text { uncertain, becoming } \\
\text { paralyzed by } \\
\text { uncertainty }\end{array}$ & 1) not acting when uncertain & $\begin{array}{l}\text { "It brings me to a halt like I can't do } \\
\text { anything” } \\
\text { "It's almost as if my life comes to a } \\
\text { standstill” }\end{array}$ \\
\hline Problem Solve & $\begin{array}{l}\text { Thinking about } \\
\text { several possible } \\
\text { solutions with the } \\
\text { intention of taking } \\
\text { action and } \\
\text { implementing a } \\
\text { solution or action } \\
\text { step }\end{array}$ & $\begin{array}{l}\text { 1) considering options in the spirit of } \\
\text { carrying out a solution when } \\
\text { uncertain or } \\
\text { 2) implementing a solution when } \\
\text { uncertain }\end{array}$ & $\begin{array}{l}\text { "I thought I will see if I can rearrange the } \\
\text { paying one and I did and it worked out } \\
\text { well" } \\
\text { "if I've encountered it before I'll probably } \\
\text { think about what I did then and if it } \\
\text { worked" }\end{array}$ \\
\hline Overprepare & $\begin{array}{l}\text { Doing more than is } \\
\text { typical of people to }\end{array}$ & $\begin{array}{l}\text { 1) explicitly says they overprepared } \\
\text { when uncertain }\end{array}$ & $\begin{array}{l}\text { "I ended up flying here to find a place } \\
\text { because I couldn't deal with not knowing" }\end{array}$ \\
\hline
\end{tabular}




\begin{tabular}{|c|c|c|c|}
\hline & $\begin{array}{l}\text { resolve or be ready } \\
\text { for uncertainty; } \\
\text { includes excessive } \\
\text { information seeking }\end{array}$ & $\begin{array}{l}\text { 2) says they did more than they } \\
\text { needed to when uncertain } \\
\text { 3) uses excessive measures (in terms } \\
\text { of time or effort) to resolve } \\
\text { uncertainty } \\
\text { Note: Code each separate behaviour } \\
\text { for this theme }\end{array}$ & $\begin{array}{l}\text { "definitely the night before when I was } \\
\text { reading through a few things doing some } \\
\text { research, I realized I probably don't have } \\
\text { to be doing all this.” }\end{array}$ \\
\hline $\begin{array}{l}\text { Check things over } \\
\text { repeatedly }\end{array}$ & $\begin{array}{l}\text { Going over } \\
\text { something more than } \\
\text { once }\end{array}$ & $\begin{array}{l}\text { 1) checking more than once when } \\
\text { uncertain }\end{array}$ & $\begin{array}{l}\text { "Of course double checking, triple } \\
\text { checking" } \\
\text { "I was repeatedly looking at the } \\
\text { information to make sure it was all } \\
\text { correct" }\end{array}$ \\
\hline $\begin{array}{l}\text { Seek reassurance from } \\
\text { others }\end{array}$ & $\begin{array}{l}\text { Soliciting opinions } \\
\text { from others about: } \\
\text { 1) how an uncertain } \\
\text { situation will resolve } \\
\text { 2) that things will be } \\
\text { 'okay' } \\
\text { 3) that they did } \\
\text { things well/correctly }\end{array}$ & $\begin{array}{l}\text { 1) reassurance seeking when } \\
\text { uncertain as per definition }\end{array}$ & $\begin{array}{l}\text { "I'd be like 'I don't know if I could do it } \\
\text { yet', and like my boyfriend would be like } \\
\text { 'no, you could do it, like you're okay’”. }\end{array}$ \\
\hline Reassure self & $\begin{array}{l}\text { Telling oneself that } \\
\text { it will be okay or use } \\
\text { other reassuring } \\
\text { statements }\end{array}$ & 1) reassuring self as per definnition & $\begin{array}{l}\text { "I talk to myself and say okay you can do } \\
\text { this or you are okay" }\end{array}$ \\
\hline \multicolumn{4}{|c|}{ Question 2. Function of Behavioural Responses to Uncertainty } \\
\hline Code & Definition & $\begin{array}{l}\text { Coding Rules } \\
\text { (Code when a participant } \\
\text { describes...) }\end{array}$ & Examples \\
\hline To reduce distress & $\begin{array}{l}\text { The behaviour serves } \\
\text { to improve the }\end{array}$ & $\begin{array}{l}\text { 1) a behaviour reduces negative } \\
\text { affect (e.g., distress, anxiety, } \\
\text { discomfort, stress) }\end{array}$ & $\begin{array}{l}\text { "ummm when I do start preparing, I think } \\
\text { the reason I do it is because it does calm } \\
\text { me down, it reduces the anxiety and } \\
\text { reduces the sense or the I guess the }\end{array}$ \\
\hline
\end{tabular}




\begin{tabular}{|c|c|c|c|}
\hline & $\begin{array}{l}\text { person's emotional } \\
\text { state }\end{array}$ & $\begin{array}{l}\text { 2) a behaviour increases positive } \\
\text { affect or feelings (e.g., confidence, 'I } \\
\text { feel better') }\end{array}$ & $\begin{array}{l}\text { looming of the uncertainty. So that's } \\
\text { probably why I do it." }\end{array}$ \\
\hline To increase certainty & $\begin{array}{l}\text { The behaviour serves } \\
\text { to help the person } \\
\text { feel more certain }\end{array}$ & $\begin{array}{l}\text { 1) a behaviour increases certainty } \\
\text { 2) a behaviour reduces uncertainty }\end{array}$ & $\begin{array}{l}\text { "so uh, just um, I'm following their } \\
\text { advice, so that does help ease the } \\
\text { uncertainty of it sometimes" }\end{array}$ \\
\hline $\begin{array}{l}\text { To feel prepared for the } \\
\text { worst }\end{array}$ & $\begin{array}{l}\text { The behaviour serves } \\
\text { to help the person } \\
\text { feel ready for a bad } \\
\text { scenario }\end{array}$ & $\begin{array}{l}\text { 1) a behaviour improving feelings of } \\
\text { preparation in relation to a potential } \\
\text { negative outcome }\end{array}$ & $\begin{array}{l}\text { "Just wanting to be prepared, just in case. } \\
\text { Even though I know there is a good } \\
\text { chance that it won't happen if it does I } \\
\text { need to know what to do in that situation." }\end{array}$ \\
\hline To optimize outcomes & $\begin{array}{l}\text { The behaviour serves } \\
\text { to help the person } \\
\text { feel like they will get } \\
\text { the best result or the } \\
\text { best outcome } \\
\text { possible }\end{array}$ & $\begin{array}{l}\text { 1) a behaviour improving feelings of } \\
\text { preparation or certainty in relation to } \\
\text { a potential positive outcome or } \\
\text { desirable state }\end{array}$ & $\begin{array}{l}\text { "Well I need to make sure I have all the } \\
\text { information so I can do the best job } \\
\text { possible, so I can be certain that like I did } \\
\text { the best I could with all the information } \\
\text { that was provided so I have to double } \\
\text { check the information so I am sure I really } \\
\text { know it" }\end{array}$ \\
\hline $\begin{array}{l}\text { To elicit support from } \\
\text { others }\end{array}$ & $\begin{array}{l}\text { The behaviour serves } \\
\text { to elicit feelings of } \\
\text { being helped by } \\
\text { others }\end{array}$ & $\begin{array}{l}\text { 1) a behaviour providing feelings of } \\
\text { support from or connection to others } \\
\text { 2) a behaviour eliciting help from } \\
\text { others }\end{array}$ & $\begin{array}{l}\text { "Probably needing reassurance. Just to } \\
\text { like if I think what I did was a good idea. } \\
\text { I like to make sure that other people agree } \\
\text { with me just for like support, to support } \\
\text { my own thoughts kind of thing." }\end{array}$ \\
\hline
\end{tabular}




\begin{tabular}{|c|c|c|c|}
\hline \multicolumn{4}{|c|}{ Question 3a. Cessation Factors (General) } \\
\hline Code & Definition & $\begin{array}{l}\text { Coding Rules } \\
\text { (Code when a participant } \\
\text { describes...) }\end{array}$ & Examples \\
\hline Uncertainty resolves & $\begin{array}{l}\text { The behaviour } \\
\text { ceases when an } \\
\text { uncertain situation } \\
\text { resolves in a definite } \\
\text { outcome }\end{array}$ & $\begin{array}{l}\text { 1) stopping a behaviour at the point } \\
\text { that they become certain about an } \\
\text { outcome }\end{array}$ & "if the outcome is positive then you stop" \\
\hline No longer possible & $\begin{array}{l}\text { The behaviour } \\
\text { ceases when it } \\
\text { becomes impossible } \\
\text { for the person to } \\
\text { engage in it } \\
\text { anymore; or there is } \\
\text { no indication that the } \\
\text { behaviour stops if it } \\
\text { is possible to } \\
\text { continue }\end{array}$ & $\begin{array}{l}\text { 1) stopping a behaviour when } \\
\text { something prevents its continuation } \\
\text { (e.g., a deadline prevents additional } \\
\text { checking; there are no additional } \\
\text { people to ask for reassurance) } \\
\text { 2) continuing a behaviour if it is } \\
\text { possible to do so }\end{array}$ & $\begin{array}{l}\text { "yeah because each person is different. } \\
\text { You get as many opinions as you can" }\end{array}$ \\
\hline Once or twice & $\begin{array}{l}\text { The behaviour } \\
\text { ceases after it is } \\
\text { carried out } 1-2 \text { times }\end{array}$ & $\begin{array}{l}\text { 1) stopping a behaviour after } \\
\text { completing it } 1-2 \text { times }\end{array}$ & $\begin{array}{l}\text { "I guess I just have a heuristic of like it } \\
\text { check it over once...And if I figure I } \\
\text { don’t need to do it again” }\end{array}$ \\
\hline
\end{tabular}




\begin{tabular}{|c|c|c|c|}
\hline $\begin{array}{l}\text { Subjective feeling to } \\
\text { stop }\end{array}$ & $\begin{array}{l}\text { The behaviour } \\
\text { ceases when the } \\
\text { person experiences a } \\
\text { subjective sense that } \\
\text { the behaviour has } \\
\text { been done enough }\end{array}$ & $\begin{array}{l}\text { 1) stopping a behaviour after } \\
\text { experiencing a subjective feeling to } \\
\text { stop } \\
\text { 2) stopping a behaviour after a } \\
\text { subjective positive shift in feelings } \\
\text { 3) stopping a behaviour after a } \\
\text { subjective feeling that one has } \\
\text { enough information }\end{array}$ & "it just comes when it feels right" \\
\hline $\begin{array}{l}\text { No longer improves } \\
\text { emotion or situation }\end{array}$ & $\begin{array}{l}\text { The behaviour } \\
\text { ceases because it } \\
\text { stops producing } \\
\text { positive feelings or } \\
\text { stops improving the } \\
\text { outcome of a } \\
\text { situation }\end{array}$ & $\begin{array}{l}\text { 1) stopping a behaviour if it no } \\
\text { longer improves ones feelings } \\
\text { 2) stopping a behaviour if it no } \\
\text { longer improves the outcome of a } \\
\text { situation }\end{array}$ & $\begin{array}{l}\text { "if they disagree then it makes me feel } \\
\text { more uncertain and I don't want to hear } \\
\text { about it” }\end{array}$ \\
\hline \multicolumn{4}{|c|}{ Question 3b. Cessation Factors (Worry) } \\
\hline Code & Definition & $\begin{array}{l}\text { Coding Rules } \\
\text { (Code when a participant } \\
\text { describes...) }\end{array}$ & Examples \\
\hline Uncertainty resolves & $\begin{array}{l}\text { Worry ceases only } \\
\text { when an uncertain } \\
\text { situation resolves in } \\
\text { a definite outcome }\end{array}$ & $\begin{array}{l}\text { 1) worrying until they know the } \\
\text { outcome } \\
\text { 2) stopping worry only when the } \\
\text { outcome is known }\end{array}$ & $\begin{array}{l}\text { "I worry until the uncertain situation } \\
\text { resolves." }\end{array}$ \\
\hline Take action & $\begin{array}{l}\text { Worry ceases when a } \\
\text { person engages in a } \\
\text { behaviour aimed at } \\
\text { reducing the worry }\end{array}$ & $\begin{array}{l}\text { 1) stopping worry when an action is } \\
\text { taken (note: includes steps a person } \\
\text { can take 'in their head'; covert } \\
\text { actions) }\end{array}$ & $\begin{array}{l}\text { "Taking action for me relives the anxiety } \\
\text { and the stress." }\end{array}$ \\
\hline
\end{tabular}




\begin{tabular}{|c|c|c|c|}
\hline Distract myself & $\begin{array}{l}\text { Worry ceases when a } \\
\text { person diverts their } \\
\text { attention with a } \\
\text { distraction }\end{array}$ & $\begin{array}{l}\text { 1) stopping worry when engaging in } \\
\text { a distracting activity } \\
\text { 2) stopping worry when distracted } \\
\text { (i.e., distraction is not introduced by } \\
\text { the person but comes from the } \\
\text { environment) }\end{array}$ & $\begin{array}{l}\text { "Trying to occupy my mind with } \\
\text { something else." }\end{array}$ \\
\hline $\begin{array}{l}\text { Reassurance from } \\
\text { others }\end{array}$ & $\begin{array}{l}\text { Worry ceases when a } \\
\text { person receives } \\
\text { reassurance from } \\
\text { others }\end{array}$ & $\begin{array}{l}\text { 1) stopping worry by seeking } \\
\text { reassurance from others }\end{array}$ & $\begin{array}{l}\text { "talking to my parents and family...my } \\
\text { mom is always like don’t worry, you'll } \\
\text { get a job, so there’s that reassurance” }\end{array}$ \\
\hline Reassure myself & $\begin{array}{l}\text { Worry ceases when a } \\
\text { person reassures } \\
\text { him/herself }\end{array}$ & $\begin{array}{l}\text { 1) stopping worry by saying a } \\
\text { reassuring statement to oneself } \\
\text { 2) stopping worry by telling oneself } \\
\text { to stop worrying }\end{array}$ & $\begin{array}{l}\text { "I'll then reassure myself, think about it } \\
\text { slowly, and then I'll go to sleep.... When } \\
\text { I wake up that worry is no longer there." }\end{array}$ \\
\hline \multicolumn{4}{|c|}{ Question 4. Problems caused by Behaviours } \\
\hline Code & Definition & $\begin{array}{l}\text { Coding Rules } \\
\text { (Code when a participant } \\
\text { describes...) }\end{array}$ & Examples \\
\hline $\begin{array}{l}\text { Cognitive/emotional } \\
\text { distress }\end{array}$ & $\begin{array}{l}\text { Behaviours cause } \\
\text { problems by leading } \\
\text { to thinking patterns } \\
\text { or emotions that the } \\
\text { person finds } \\
\text { distressing }\end{array}$ & $\begin{array}{l}\text { 1) behaviours causing problems } \\
\text { because of thinking patterns that are } \\
\text { upsetting } \\
\text { 2) behaviours causing problems } \\
\text { because of feelings that are upsetting }\end{array}$ & $\begin{array}{l}\text { "it bugs me that I'm always thinking } \\
\text { about things” }\end{array}$ \\
\hline Work/School problems & $\begin{array}{l}\text { Behaviours cause } \\
\text { problems by leading } \\
\text { to problems at work } \\
\text { or in school }\end{array}$ & $\begin{array}{l}\text { 1) behaviours causing problems } \\
\text { related to school } \\
\text { 2) behaviours causing problems } \\
\text { related to work }\end{array}$ & $\begin{array}{l}\text { "Procrastination definitely does have a } \\
\text { huge effect on my marks" }\end{array}$ \\
\hline Relationship problems & $\begin{array}{l}\text { Behaviours cause } \\
\text { problems by leading } \\
\text { to problems in } \\
\text { relationships }\end{array}$ & $\begin{array}{l}\text { 1) behaviours causing problems in } \\
\text { relationships }\end{array}$ & "I piss people off" \\
\hline
\end{tabular}




\begin{tabular}{|c|c|c|c|}
\hline $\begin{array}{l}\text { Lack of life balance / } \\
\text { low engagement in } \\
\text { pleasant activities }\end{array}$ & \begin{tabular}{|l|} 
Behaviours cause \\
problems by leading \\
to avoidance of \\
activities of interest \\
or interfering with \\
carrying out pleasant \\
activities \\
\end{tabular} & $\begin{array}{l}\text { 1) behaviours causing problems by } \\
\text { leading to avoidance of activities of } \\
\text { interest } \\
\text { 2) behaviours causing problems by } \\
\text { leading to few pleasure activities or a } \\
\text { sense of low quality of life } \\
\text { 3) behaviours cause problems by } \\
\text { taking up excessive time }\end{array}$ & $\begin{array}{l}\text { "Yeah a lot of the times I avoid getting } \\
\text { involved with things, what I mean } \\
\text { involved I mean other groups and sort of } \\
\text { volunteering or different social sort of } \\
\text { things." }\end{array}$ \\
\hline $\begin{array}{l}\text { Physical ailments (e.g., } \\
\text { headaches, muscle } \\
\text { pain) }\end{array}$ & $\begin{array}{l}\text { Behaviours cause } \\
\text { problems by leading } \\
\text { to physical health } \\
\text { problems }\end{array}$ & $\begin{array}{l}\text { 1) behaviours causing physical } \\
\text { health problems } \\
\text { 2) behaviours negatively impacting } \\
\text { health }\end{array}$ & $\begin{array}{l}\text { "it affects me physically and I'm prone to } \\
\text { headaches and migraines because of the } \\
\text { tension" }\end{array}$ \\
\hline
\end{tabular}

\section{General Coding Guidelines:}

1. You do not need to code everything - code only what you believe fits for sure into the codes/categories

2. Code only participant answers (do not code what the interviewer says)

a. Exception: if participants says "mmm hmm" or "ya" but they do not reference the code, then code the interviewer phrase they agreed with along with the participant's phrase of agreement.

3. Code only the first phrase that a participant says that "makes it" into the code. Once a code has been made for a participant, no need to code additional phrases that are the same code.

4. Code phrases - phrase may start at the beginning of a sentence, and may end when the thought is complete (if it were proper writing, there could be a "comma" after it), or it may end at the end of a sentence. Stop coding when the extra parts of the sentence are no longer necessary for the code (i.e., the thought would already "make it" into the code without any extra information).

5. If, after a conversation, the participant changes their mind/stance on something discussed, do not code prior contradictory information, and code the final idea that they decide on (e.g., at first participant says that probability does not moderate their response to uncertainty, but after discussion they say that probability DOES moderate their response to uncertainty, code only that probability does moderate). 


\section{Appendix D -Informed Consent Agreements}

\section{Consent Agreement - Studies 1a and 1b}

Title of Study: Interview Study

You are being asked to participate in a research study. Before you give your consent to be a volunteer, it is important that you read the following information and ask as many questions as necessary to be sure you understand what you will be asked to do.

\section{Investigators:}

Katie Fracalanza, MA, Graduate Student, Department of Psychology, Ryerson University Naomi Koerner, PhD, Assistant Professor, Department of Psychology, Ryerson University Kelly McShane, PhD, Assistant Professor, Department of Psychology, Ryerson University Martin Antony, PhD, Professor, Department of Psychology, Ryerson University

Purpose of the Study: The purpose of this study is to learn more about how people react to uncertainty and the reasons for those responses. Currently there is theory and research about how people react to uncertainty, but this has been investigated mainly through the use of questionnaires that use rating scales to measure people's responses. This study seeks to expand on knowledge from questionnaires by using an interview, during which people will respond to questions in their own words. For example, you will be asked to describe how you respond when you are in uncertain situations, and how you feel about uncertainty.

Description of the Study: The study will involve 1 visit to the Psychology Research and Training Centre at Ryerson University, located at 105 Bond Street. The total time commitment for this study will be approximately 3 hours. During this visit you will complete an interview about your responses to uncertainty, and your answers will be audio taped with a recorder. Next, you will be asked to complete a battery of questionnaires. You will earn $\$ 40$ for completing this study.

This study is being conducted to fulfill the requirements of a doctoral degree in Psychology at Ryerson University.

Potential Risks or Discomforts: There is minimal risk involved if you agree to take part in this study. You may experience some discomfort while answering questions in interview or questionnaire format. You may also begin to experience fatigue during your visit. If you begin to feel uncomfortable, you may discontinue participation or take a break from the study and return later. If you decide to stop participating, you will still be entitled to compensation for the parts of the study that you started or completed prior to discontinuing participation (i.e., \$25 for the responses to uncertainty interview, and \$15 for the battery of questionnaires). 
Potential Benefits of the Study To You or Others: We cannot guarantee that you will receive any benefits from participating in this study. You may derive benefit from thinking about your answers to the questions that you are asked as part of this study, as it may increase your awareness of your emotions and behaviours. You may also develop a better understanding of research methodology. You will be providing researchers with valuable insight about the way that people experience uncertainty. This will inform current theories about the connection between how people experience uncertainty and worry. This research may also lead to improvements in interventions for people who experience difficulty managing their worry.

Confidentiality: Confidentiality will be respected and no information that discloses your identity will be released or published without consent, unless required by law. Everything you disclose in this study will remain completely confidential within the people who are directly involved in the study; however, there are five specific cases in which I may need to break confidentiality. These are:

(1) if you intend to harm yourself;

(2) if you intend on harming someone else;

(3) if there is reasonable suspicion that a child up to the age of 16 years is at risk of neglect or abuse, we are required by law to report this to the Children's Aid Society right away;

(4) if our files are subpoenaed by the courts (records can be opened by a specific court order); (5) if a regulated health professional has engaged in inappropriate sexual behavior toward you and you provide us with the name of this individual, we are obligated to report them to their regulatory body.

This informed consent agreement and all data that identifies you will be stored in a locked storage space in the Psychology Research and Training Centre. An ID number as opposed to your name will be used on all forms and questionnaires that you complete, on the interviews that you take part in, and on all electronic files that contain data that you generate during the study.

Any data that you generate while participating in this study will be kept in a locked file cabinet, separate from this consent agreement and any data that identifies you. Your responses to the responses to uncertainty interview will be audio-taped and the electronic audio files will be password protected, accessible only to study investigators and individuals who are supervised by the main study investigator, and stored on a secure network. These audio files will be transcribed into an electronic document by the main study investigator or individuals who are supervised by the main study investigator. The transcribed documents will be password protected and saved on a secure network. Only study investigators and individuals involved in assisting with running this study will have access to any data that you provide during the course of this study. Your consent form and all data will be kept for seven years after the publication of this research. Your confidentiality will be protected to the full extent allowed by law. Quotes that you provide during the interview may be included in publications, although your name and any other identifying information will not be provided with the quotes.

Compensation for Participation: You will earn $\$ 40$ for completing this study. You are asked to arrange transportation for yourself to the Psychology Research and Training Centre at Ryerson University. You will not be paid for the telephone screen that you completed to determine your eligibility for the study. 
Voluntary Nature of Participation: Participation in this study is voluntary. Your choice of whether or not to participate will not influence your future relations with Ryerson University. If you decide to participate, you are free to withdraw your consent and to stop your participation at any time without penalty or loss of benefits to which you are allowed. Your right to withdraw your consent also applies to our use of your data. If you decide that you do not want us to keep or analyze data that you have provided during the course of your participation in this study, please feel free to notify us.

At any point in the study, you may refuse to answer any question or stop participation altogether.

Questions about the Study: If you have any questions about the research now, please ask. If you have questions later about the research, you may contact Katie Fracalanza, MA, Graduate Student, Department of Psychology, Ryerson University, 416-979-5000 extension 2182. You may also contact Dr. Naomi Koerner, PhD, Department of Psychology, Ryerson University, 416979-5000 extension 2151.

If you have questions regarding your rights as a participant in this study, you may contact Toni Fletcher at the Ryerson University Research Ethics Board for information.

Toni Fletcher

Research Ethics Coordinator

Ryerson University, 350 Victoria Street, Room YDI 1100

Toronto, Ontario, Canada M5B 2K3

Phone: (416) 979-5000 Ext. 7112

Email: toni.fletcher@ryerson.ca

Web: http://www.ryerson.ca/about/vpresearch/ethics.html

Agreement: Your signature below indicates: (1) that you have read the information in this agreement and have had a chance to ask any questions you have about the Interview Study; (2) that you agree that information collected from you during the telephone screen for the Interview Study can be retained and analyzed; and (3) that you agree to be in the Interview Study (as described in this consent form) and have been told that you can change your mind and withdraw your consent to participate at any time. You have been given a copy of this agreement. You have been told that by signing this consent agreement you are not giving up any of your legal rights.

Name of Participant (please print)

Signature of Participant

Date

Signature of Investigator

Date 
Your signature below indicates that you have read and understand that you will be audiorecorded for only the purposes of this study. Your signature indicates that you agree to be audiorecorded and have been told that you can change your mind and withdraw this consent at any time.

Name of Participant (please print)

Signature of Participant

Signature of Investigator
Date

Date

In addition to the current study, it is possible that you may be eligible for other studies that are being conducted by our research team at Ryerson University. Please initial beside Option 1 below if you are interested in receiving information about additional studies that you may be eligible for currently or in the future. Please initial beside Option 2 if you are not interested in receiving information about other studies.

Option 1. Initial here if you would like to be contacted by e-mail or telephone about studies that you may be eligible for that are being run by our research team currently or in the future. You will only be contacted regarding potential participation in studies, and your contact information will not be used for any other purposes.

If you would like to be contacted about other studies that are being run by our research team currently or in the future, please provide your contact information below. Your contact information will be stored in a password protected electronic spreadsheet on the network drive associated with our lab. Only people who are running studies in our lab will have access to this spreadsheet.

Name:

E-mail:

Telephone \#:

Option 2. Initial here if you wish not to receive information about other studies that are being run by our research team currently or in the future. 


\section{Consent Agreement - Study 2}

Title of Study: Uncertainty Training Study

You are being asked to participate in a research study. Before you give your consent to be a volunteer, it is important that you read the following information and ask as many questions as necessary to be sure you understand what you will be asked to do. This study will partially fulfill the requirements of Katie Fracalanza's doctoral degree in Psychology at Ryerson University.

\section{Investigators:}

Katie Fracalanza, MA, Graduate Student, Department of Psychology, Ryerson University Naomi Koerner, PhD, Assistant Professor, Department of Psychology, Ryerson University Martin Antony, PhD, Professor, Department of Psychology, Ryerson University Kelly McShane, PhD, Assistant Professor, Department of Psychology, Ryerson University

Purpose of the Study: Although research suggests that there is a link between uncertainty and well-being, we do not have a good understanding of the effects that intentionally approaching uncertainty has on the moods, thoughts and behaviour of people who have difficulty managing their worry.

Description of the Study: The study will involve 3 visits to the Psychology Research and Training Centre at Ryerson University, located at 105 Bond Street.

For this study, you will be randomly assigned to 1 of 2 experimental conditions.

\section{$\underline{\text { Visit 1: }}$}

\section{$\underline{\text { Part A }}$}

During Visit 1, all participants (assigned to condition 1 or condition 2) will be asked to complete a battery of questionnaires.

You will also learn how to complete a diary form on which you will rate things like your level of worry, specific behaviours, and feelings about uncertainty. You will be emailed a link to this diary form every day (even on days when you do not visit the lab), beginning on Visit 1 and ending on Visit 3 (i.e., every day for 2 weeks). You will be asked to follow this emailed link and to complete and submit the diary form electronically every day for 2 weeks. If you do not have internet or computer access, you have the option to complete the diary form in paper-and-pencil format each day for 2 weeks. The diary form takes about 5 minutes to complete each day. You will be compensated $\$ 15$ at the end of visit 3 for completing this task every day for 2 weeks.

Visit 1 Part A will take 60 to 90 minutes. If you are assigned to condition 1, this marks the end of Visit 1. 


\section{$\underline{\text { Part B }}$}

If you are assigned to condition 2, you will complete Visit 1 Part B, which involves receiving a training session in a technique that involves "confronting” uncertainty in your daily life. You will learn about the connection between uncertainty and worry, and you will create a plan with the experimenter about small ways that you could get more experience in dealing with uncertainty (e.g., going to a movie without looking up any reviews, sending out a low-priority email without checking it over more than once, making a minor decision without asking other people for their opinions on it, working on a project with an uncertain outcome). You will be asked to complete an activity (that involves feeling uncertain) from this plan every day for 7 days (each day in between Visit 1 and Visit 2). You will also be asked to complete a brief form each time you purposefully complete an activity that involves approaching uncertainty. You will bring these forms back to the Lab with you at Visit 2. These tasks will take approximately 60 minutes.

Participants in condition 1 and condition 2 will be compensated \$15 for completing Visit 1.

Note: Participants in condition 1 will have the opportunity to receive training in the technique that involves "confronting" uncertainty in daily life after all study tasks are complete (i.e., at the end of Visit 3).

\section{Visit 2:}

Visit 2 will be the same for participants in condition 1 and condition 2 . Visit 2 will be scheduled about 1 week after visit 1 . During visit 2 you will be asked to complete the same battery of questionnaires that you completed during visit 1, along with a few additional questionnaires. You will also return any forms to the Lab that you were asked to complete between Visit 1 and Visit 2. Visit 2 will take 60 to 90 minutes. You will be compensated $\$ 15$ for completing Visit 2.

\section{$\underline{\text { Visit 3: }}$}

Visit 3 will be the same for participants in condition 1 and condition 2 . Visit 3 will be scheduled about 1 week after Visit 2. During Visit 3 you will complete questionnaires, and return the forms that you completed outside of the Lab. Visit 3 will take 60 to 90 minutes. You will be compensated \$15 for completing Visit 3, and \$15 for completing the dairy form outside of the Lab between Visit 1 and Visit 3, for a total of $\$ 30$ at the end of Visit 3.

In summary, the total time commitment for this study will be about 4 to 5.5 hours at the Lab, and the time that it takes to fill out daily forms and complete daily activities in which you will “confront” uncertainty outside of the lab for 2 weeks. You can earn $\$ 60$ in total for completing all parts of this study.

This study is funded by the Canadian Institutes of Health Research.

Potential Risks or Discomforts: There is minimal risk involved if you agree to take part in this study. You may experience some discomfort while completing questionnaires, or while 
completing tasks that involve confronting minor uncertain situations in your own life. You do not have to answer any question that you do not feel comfortable answering, and you do not need to complete any task that you do not feel comfortable completing. You may experience fatigue during your visits to the lab. You may discontinue participation at any time, or take a break from the study and return later. If you decide to stop participating, you will still be entitled to compensation for the parts of the study that you started or completed prior to discontinuing participation (e.g., \$15 for completing Visit 1, if you decide to stop participating right after completing the tasks required for Visit 1).

Potential Benefits of the Study to You or Others: We cannot guarantee that you will receive any benefits from participating in this study. You will not receive a treatment for worry in this study. If you are interested in treatment for worry, you may ask the experimenter for treatment resources in the Greater Toronto Area. You may derive benefit from learning about the connection between uncertainty and worry, and from the exercises involving approaching uncertainty in your life, as this is thought to help people to cope with uncertainty better. You may also benefit from thinking about your answers to the questions that you are asked as part of this study, as it may increase your awareness of your emotions and behaviours. You may also develop a better understanding of research methodology. You will be providing researchers with valuable insight about the connection between uncertainty and worry. This will inform current theories about worry. This research may also lead to improvements in interventions for people who experience difficulty managing their worry.

Confidentiality: Confidentiality will be respected. Everything you disclose in this study will remain completely confidential within the people who are directly involved in the study; however, there are five specific cases in which I may need to break confidentiality. These are:

(1) if you intend to harm yourself;

(2) if you intend on harming someone else;

(3) if there is reasonable suspicion that a child up to the age of 16 years is at risk of neglect or abuse, we are required by law to report this to the Children's Aid Society right away;

(4) if our files are subpoenaed by the courts (records can be opened by a specific court order);

(5) if a regulated health professional has engaged in inappropriate sexual behavior toward you

and you provide us with the name of this individual, we are obligated to report them to their regulatory body.

This informed consent agreement and all data that identifies you will be stored in a locked storage space in the Psychology Research and Training Centre. An ID number as opposed to your name will be used on the phone interview in which you took part prior to coming to the Psychology Research and Training Centre, on all questionnaires you complete, and in all computer files that contain the data you generate during the study. Your phone interview and questionnaires will be kept in a locked file cabinet, separate from this consent agreement and any identifying information. This consent form, the notes from your telephone interview and the questionnaires will be kept for 7 years after the publication of this research, after which they will be shredded. Your confidentiality will be protected to the full extent allowed by law. Only group findings will be reported in publications and presentations arising from this research. 
The responses that you provide to questions on the dairy form that is emailed to you each day will be stored using computer software called Qualtrics. The data are securely and confidentially stored on a remote server and you will be identified by number only. Please note that because the data are securely stored on a USA based server (Qualtrics), it is subject to the Patriot Act. If you care to know more about this, please visit the following link: http://epic.org/privacy/terrorism/hr3162.html. Under the Patriot Act, stored data may be intercepted in rare cases if United States officials have a reason to believe the data contains information related to suspected terrorism. However, your name is not stored with your questionnaire data, and therefore would not be available to these officials.

Compensation for Participation: You will earn $\$ 60$ for completing this study. You are asked to arrange transportation for yourself to the Psychology Research and Training Centre at Ryerson University. You will not be paid for the telephone screen that you completed to determine your eligibility for the study.

Voluntary Nature of Participation: Participation in this study is voluntary. Your choice of whether or not to participate will not influence your future relations with Ryerson University. If you decide to participate, you are free to withdraw your consent and to stop your participation at any time without penalty or loss of benefits to which you are allowed. Your right to withdraw your consent also applies to our use of your data. If you decide that you do not want us to keep or analyze the data that you provided during the course of your participation in this study, and you notify us of this before the end of your participation in this study (i.e., before the end of your third visit to the lab), we will withdraw and shred your data. We are unable to withdraw your data after you have completed your third visit to the lab because your data will not be linked to you in any way, and therefore, we will not be able to identify which data you provided.

At any point in the study, you may refuse to answer any question or stop participation altogether.

\section{Questions about the Study:}

If you have any questions about the research now, please ask. If you have questions later about the study, you may contact: 
Katie Fracalanza, MA

Main Study Investigator

Ryerson University

105 Bond Street

Toronto, ON M5B2K3

(416) 979-5000 x2182

caplab@psych.ryerson.ca
Naomi Koerner, PhD

PhD Study Supervisor

Department of Psychology

Ryerson University

350 Victoria Street

Toronto, ON M5B2K3

(416) 979-5000 x2151

naomi.koerner@psych.ryerson.ca

If you have questions about your rights as a research participant, you may contact:

Toni Fletcher

Research Ethics Coordinator, Office of the Vice President, Research and Innovation

Ryerson University

350 Victoria Street, YDI 1100, Toronto, ON M5B2K3

(416) 979-5000x7112 toni.fletcher@ryerson.ca

Agreement: Your signature below indicates: (1) that you have read the information in this agreement and have had a chance to ask any questions you have about the Uncertainty Training Study; (2) that you agree that information collected from you during the telephone screen for the Uncertainty Training Study can be retained and analyzed; and (3) that you agree to be in the Uncertainty Training Study (as described in this consent form) and have been told that you can change your mind and withdraw your consent to participate at any time. You have been given a copy of this agreement. You have been told that by signing this consent agreement you are not giving up any of your legal rights.

Name of Participant (please print)

Signature of Participant

Date

Signature of Investigator

Date 
In addition to the current study, it is possible that you may be eligible for other studies that are being conducted by our research team at Ryerson University. Please initial beside Option 1 below if you are interested in receiving information about additional studies that you may be eligible for currently or in the future. Please initial beside Option 2 if you are not interested in receiving information about other studies.

Option 1. Initial here if you would like to be contacted by e-mail or telephone about studies that you may be eligible for that are being run by our research team currently or in the future. You will only be contacted regarding potential participation in studies, and your contact information will not be used for any other purposes.

If you would like to be contacted about other studies that are being run by our research team currently or in the future, please provide your contact information below. Your contact information will be stored in a password protected electronic spreadsheet on the network drive associated with our lab. Only people who are running studies in our lab will have access to this spreadsheet.

Name:

E-mail:

Telephone \#:

Option 2. Initial here if you wish not to receive information about other studies that are being run by our research team currently or in the future. 


\section{Appendix E - Demographics Questionnaire}

ID\#:

Sex:

$\square$ Female

$\square$ Male

Age:

Marital Status:

$\square$ Married/Common Law

$\square$ Single

Divorced/Widowed

Race/Ethnicity:

$\square$ Aboriginal (Inuit, Métis, North American Indian)

Arab/West Asian (e.g., Armenian, Egyptian, Iranian, Lebanese, Moroccan)

$\square$ Black (e.g., African, Haitian, Jamaican, Somali)

East Asian (e.g., Chinese, Japanese, Korean)

$\square$ Latin American

South Asian

$\square$ South East Asian

$\square$ White (Caucasian)

Mixed (please specify):

$\square$ Other (an ethnicity not listed above):

Are you currently receiving psychotherapy?

$\square$ Yes

$\square$ No

Have you received psychotherapy (e.g., cognitive behavioural therapy, supportive therapy, relaxation) in the past?

$\square$ Yes If yes, please specify the type and when you last received

$\square$ No psychotherapy: 
Are you currently taking any medications related to your psychological health (e.g., sleep, anxiety)?

$\square$ Yes $\quad$ If yes, please specify the type and dose:
$\square$ No

Are you enrolled in an educational program?

$\square$ Yes

$\square$ No

If you are enrolled in an education program, please check which one:

$\square$ College

$\square$ University

$\square$ Adult Education

Please indicate your field of Study:

If you are not enrolled in an educational program, please indicate your highest level of education:

$\square$ Some High School

$\square$ High School Diploma

$\square$ College Diploma

$\square$ Undergraduate Degree

$\square$ Masters Degree

$\square$ Doctoral Degree

Employment Status:

Not Working

$\square$ Working Part-Time

Working Full-Time

If working part-time or full-time, please indicate your occupation: 


\section{Appendix F - Debriefing Forms}

\section{Debriefing Form - Studies 1a and 1b}

Background of the Study: Studying people's responses to uncertainty is of interest because even though most people prefer certainty to uncertainty, prior research has shown that people who worry a lot tend to dislike uncertainty more than others. There are several unanswered questions about how and why people who tend to worry a lot also tend to dislike uncertainty more than the average person.

Purpose of the Study: This study investigates how people think, feel, and act when uncertain. This study also seeks to understand the reasons why people react the way that they do when uncertain. In addition, this study aims to understand any potential differences in the responses to uncertainty of people who worry a lot compared to people who do not worry a lot. The knowledge gained from this study will inform current theory about how worry is maintained over time, and will help to advance our understanding of the ways that chronic worry can be alleviated. Your willingness to participate in this study is greatly appreciated.

Resources: We provide everyone who completes this study with the same list of resources, in case they are interested in learning more about worry or anxiety. Our list of resources has titles of books on worry management, as well as referral sources (please turn over this page for the list).

Contact Information: If you have any questions or concerns about this study or your participation in this study you may contact:

Katie Fracalanza, MA

Main Study Investigator

Ryerson University

105 Bond Street

Toronto, ON M5B2K3

(416) 979-5000 x2182

caplab@psych.ryerson.ca
Naomi Koerner, PhD

PhD Study Supervisor

Department of Psychology

Ryerson University

350 Victoria Street

Toronto, ON M5B2K3

(416) 979-5000 x2151

naomi.koerner@psych.ryerson.ca
Toni Fletcher

Research Ethics Coordinator, Office of the Vice President, Research and Innovation Ryerson University 350 Victoria Street, YDI 1100, Toronto, ON M5B2K3

(416) 979-5000 x7112 toni.fletcher@ryerson.ca

If you would like any information about the results of the study once it is completed, please contact Katie Fracalanza.

A note about disclosure: In order to maintain the integrity of this research, we ask that you not disclose the purpose of this experiment to others who may be interested in taking part in this study. When participants have too much prior knowledge about the purpose of an experiment, this can affect how they behave in the experiment and the data for that person may not be usable.

\section{Thank you very much for participating in this study!}




\section{$\underline{\text { Self-Help Books for Worry }}$}

Gyoerkoe, K.L., \& Wiegartz, P.S. (2006). 10 simple solutions to worry: How to calm your mind, relax your body, \& reclaim your life. Oakland, CA: New Harbinger.

Meares, K., \& Freeston, M. (2008). Overcoming worry: A self-help guide using cognitive behavioral techniques. New York: Basic Books.

Greenberger, D., \& Padesky, C. A. (1995). Mind Over Mood. New York, NY: Guilford Press.

Other anxiety resources are available at: http://www.martinantony.com/resources

\section{Referrals in Toronto Area if Seeking Help for Worry}

\section{No Cost or Low-Cost Referrals:}

Study providing Cognitive Behavioural Therapy for Worry (to eligible participants)

Ryerson University and York University

For more information contact

Tel: 416-736-2100 ext. 33605 or anxlab@yorku.ca

Anxiety Disorders Clinic

Centre for Addiction and Mental Health

250 College Street, Toronto

Tel: 416-979-6819

Adult Mental Health Program

Humber River Regional Hospital, Toronto

Contact: Heather Wheeler, $\mathrm{PhD}$

Tel: 416-658-2003

Ryerson University Centre for Student Development and Counseling (Available to Ryerson Students Only)

350 Victoria St., Room JOR-07C, Lower Ground Floor, Jorgenson Hall, Toronto

Tel: 416-979-5195

Private Psychology Referrals:

CBT Associates of Toronto

100 Adelaide St. West, Suite 805, Toronto

Tel: 416-363-4228

Web: http://www.cbtassociates.net/
Clinic on Dupont

101 Dupont Street, Toronto

Tel: 416-515-2649

Web: http://www.theclinicondupont.com 


\section{Debriefing Form - Study 2}

Background of the Study: People who are prone to worry also tend to try to control and avoid uncertainty. Based on this, it is thought that seeking out uncertainty gradually and repeatedly might have a positive effect on worry, but this has not yet been studied in detail.

Purpose of the Study: This study investigates how learning about uncertainty and repeatedly seeking out minor uncertain situations impacts feelings, thoughts, and behaviours. We are studying this by comparing the effect of teaching people about uncertainty and asking them to approach it in small ways (condition 2) to asking people to complete assessments only (condition 1). The knowledge gained from this study will inform current theory about how worry is maintained over time, and will help to advance our understanding of the ways that chronic worry can be alleviated. Your willingness to participate in this study is greatly appreciated.

Resources: This study was not a treatment for worry. We provide everyone who completes this study with the same list of resources, in case they are interested in learning more about worry or in seeking treatment for worry. Our list of resources has titles of books on worry management, as well as referral sources (please turn over this page for the list).

If you were in the assessment only control condition, you have the option to receive training in the technique that we are studying. This technique involves learning about uncertainty and planning to approach uncertainty in small ways. Please notify the experimenter if you would like to learn more about this technique.

Contact Information: If you have any questions or concerns about this study or your participation in this study you may contact:

Katie Fracalanza, MA

Main Study Investigator

Ryerson University

105 Bond Street

Toronto, ON M5B2K3

(416) 979-5000 x2182

caplab@psych.ryerson.ca
Naomi Koerner, $\mathrm{PhD}$

PhD Study Supervisor

Department of Psychology

Ryerson University

350 Victoria Street

Toronto, ON M5B2K3

(416) 979-5000 x2151

naomi.koerner@psych.ryerson.ca
Toni Fletcher

Research Ethics Coordinator, Office of the Vice President, Research and Innovation Ryerson University 350 Victoria Street, YDI 1100, Toronto, ON M5B2K3 (416) 979-5000 x7112 toni.fletcher@ryerson.ca

If you would like any information about the results of the study once it is completed, please contact Katie Fracalanza.

A note about disclosure: In order to maintain the integrity of this research, we ask that you not disclose the purpose of this experiment to others who may be interested in taking part in this study. When participants have too much prior knowledge about the purpose of an experiment, this can affect how they behave in the experiment and the data for that person may not be usable.

\section{Thank you very much for participating in this study!}




\section{$\underline{\text { Self-Help Books for Worry }}$}

Gyoerkoe, K.L., \& Wiegartz, P.S. (2006). 10 simple solutions to worry: How to calm your mind, relax your body, \& reclaim your life. Oakland, CA: New Harbinger.

Meares, K., \& Freeston, M. (2008). Overcoming worry: A self-help guide using cognitive behavioral techniques. New York: Basic Books.

Greenberger, D., \& Padesky, C. A. (1995). Mind Over Mood. New York, NY: Guilford Press.

Other anxiety resources are available at: http://www.martinantony.com/resources

\section{Referrals in Toronto Area if Seeking Help for Worry}

\section{No Cost or Low-Cost Referrals:}

Study providing Cognitive Behavioural Therapy for Worry (to eligible participants)

Ryerson University and York University

For more information contact

Tel: 416-736-2100 ext. 33605 or anxlab@yorku.ca

\section{Anxiety Disorders Clinic}

Centre for Addiction and Mental Health

250 College Street, Toronto

Tel: 416-979-6819

\section{Adult Mental Health Program}

Humber River Regional Hospital, Toronto

Contact: Heather Wheeler, PhD

Tel: 416-658-2003

Ryerson University Centre for Student Development and Counseling (Available to Ryerson Students Only)

350 Victoria St., Room JOR-07C, Lower Ground Floor, Jorgenson Hall, Toronto

Tel: 416-979-5195

\section{Private Psychology Referrals}

CBT Associates of Toronto

100 Adelaide St. West, Suite 805, Toronto

Tel: 416-363-4228Web: http://www.cbtassociates.net

\section{Clinic on Dupont}

101 Dupont Street, Toronto

Tel: 416-515-2649

Web: http://www.theclinicondupont.com 


\section{Appendix G - Daily Diary}

Please answer the following set of questions each day that you are involved in this study.

DAY:

1. Approximately how much time did you spend worrying today (in minutes or hours):

2. How intense was your worry today?

$0 \ldots \ldots \ldots \ldots .1 \ldots \ldots \ldots \ldots . .2 \ldots \ldots \ldots \ldots . . .3 \ldots \ldots \ldots \ldots . .4$
(Not at all Intense)
(Moderately Intense)

3. How intense was any difficulty concentrating that you may have had today?

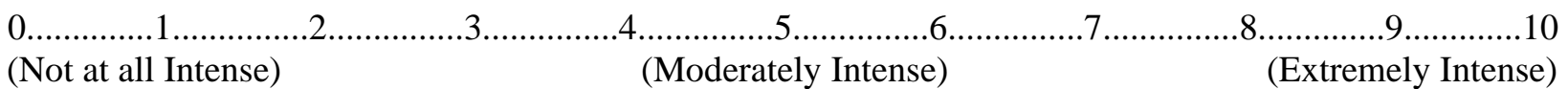

4. How intense was any difficulty sleeping that you may have had today?

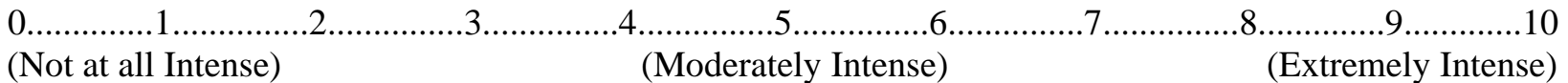

5. How intense was any muscle tension that you may have had today?

$0 \ldots \ldots \ldots \ldots .1 \ldots \ldots \ldots \ldots . .2 \ldots \ldots \ldots \ldots . . . . . . \ldots \ldots \ldots . .4$
(Not at all Intense)
(Moderately Intense)

6. How intense were any feelings of being "keyed-up” or "on edge” that you may have had today?

$0 \ldots \ldots \ldots \ldots . .1 \ldots \ldots \ldots \ldots \ldots \ldots$
(Not at all Intense)
(Moderately Intense)

7. How intense was any irritability that you may have had today?

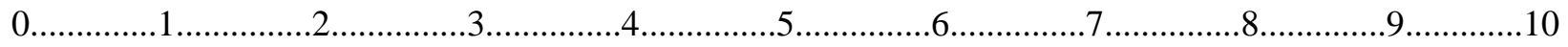
(Not at all Intense) ～(Moderately Intense) (Extremely Intense)

8. How intense was any fatigue that you may have had today?

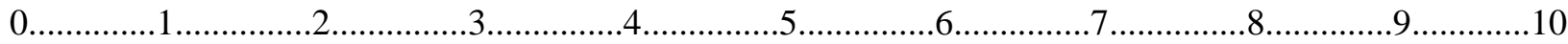

(Not at all Intense) ～(Moderately Intense) ～(Extremely Intense)


9. Uncertainty stopped me from doing things today.

$0 \ldots \ldots \ldots \ldots .1 \ldots \ldots \ldots \ldots$
(Completely Agree)
(Neither Agree nor
Disagree)

10. I felt that uncertainty was unfair today.

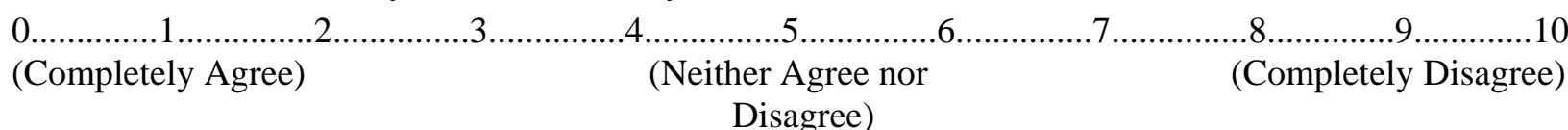

11. I felt that uncertainty spoiled things today.

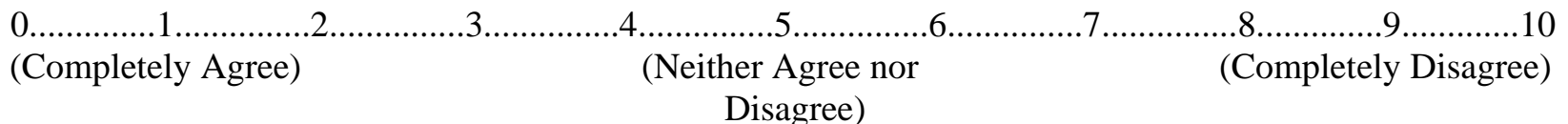

12. When I was uncertain today, it meant something bad about me.

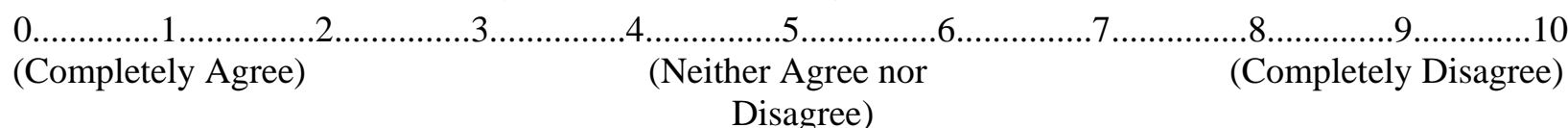

13. Uncertainty was intolerable today.

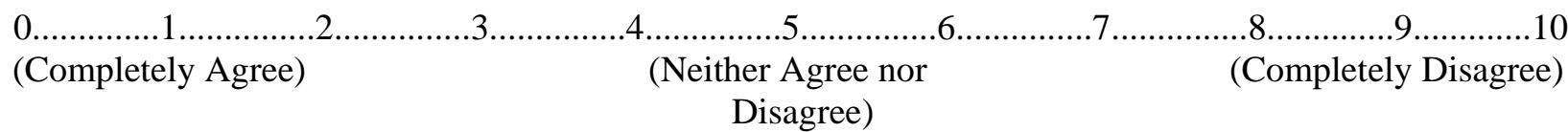

14. I felt able to cope with uncertain situations today.

$0 \ldots \ldots \ldots \ldots 1$
$\begin{gathered}\text { (Completely Agree) } \\ \text { (Neither Agree nor } \\ \text { Disagree) }\end{gathered}$

15. I felt able to cope with feeling uncertain today.

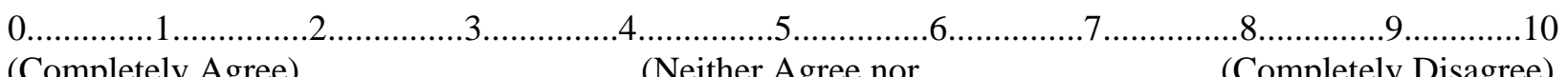




\section{References}

Abbott, B. B. (1985). Rats prefer signaled over unsignaled shock-free periods. Journal of Experimental Psychology: Animal Behavior Processes, 11, 215-223.

Abramowitz, J. S. (2009). Getting over OCD: A 10-step workbook for taking back your life. New York, NY: Guilford Press.

Abramowitz, J. S., Deacon, B. J., \& Whiteside, S. P. H. (2011). Exposure therapy for anxiety: Principles and practice. New York, NY: Guilford Press.

Adam, D. (2013). Mental health: On the spectrum. Nature, 496, 416-418.

Al-Najjar, N. I., \& Weinstein, J. (2009). The ambiguity aversion literature: A critical assessment. Economics and Philosophy, 25, 249-284.

Alexander, M. J., Haugland, G., Lin, S. P., Bertollo, D. N., \& McCorry, F. A. (2008). Mental health screening in addiction, corrections and social service settings: Validating the MMS. International Journal of Mental Health and Addiction, 6, 105-119.

American Psychiatric Association (2013). Diagnostic and statistical manual of mental disorders, $5^{\text {th }}$ ed. (DSM-5). Arlington, VA: Author.

Andrews, G., \& Hobbs, M. J. (2010). The effect of the draft DSM-5 criteria for GAD on prevalence and severity. Australian and New Zealand Journal of Psychiatry, 44, 784-790.

Andrews, G., Hobbs, M. J., Borkovec, T. D., Beesdo, K., Craske, M. G., Heimberg, R. G., . . . Stanley, M. A. (2010). Generalized worry disorder: A review of DSM-IV generalized anxiety disorder and options for DSM-V. Depression and Anxiety, 27, 134-147.

Andor, T., Gerlach, A. L., \& Rist, F. (2008). Superior perception of phasic physiological arousal and the detrimental consequences of the conviction to be aroused on worrying and metacognitions in GAD. Journal of Abnormal Psychology, 117, 193. 
Antony, M. M., Ledley, D. R., \& Heimberg R. G. (2005). Improving outcomes and preventing relapse in cognitive-behavorial therapy. New York, NY: Guilford Press.

Babrow, A. S., \& Kline, K. N. (2001). From “reducing” to “managing” uncertainty: Reconceptualizing the central challenge in breast self-exams. Social Science and Medicine, 51, 1805-1816.

Ballenger, J. C., Davidson, J. R. T., Lecrubier, Y., Nutt, D. J., Borkovec, T. D., Rickels, K., .. . Wittchen, H. (2001). Consensus statement on generalized anxiety disorder from the international consensus group on depression and anxiety. Journal of Clinical Psychiatry, 62, 53-58.

Balooch, S. B., Neumann, D. L., \& Boschen, M. J. (2012). Extinction treatment in multiple contexts attenuates ABC renewal in humans. Behaviour Research and Therapy, 50, 604609.

Baker, A., Mystkowski, J., Culver, N., Yi, R., Mortazavi, A., \& Craske, M. G. (2010). Does habituation matter? Emotional processing theory and exposure therapy for acrophobia. Behaviour Research and Therapy, 48, 1139-1143.

Bar-Anan, Y., Wilson, T. D., \& Gilbert, D. T. (2009). The feeling of uncertainty intensifies affective reactions. Emotion, 9, 123.

Barlow, D. H. (2002). Anxiety and its disorders: The nature and treatment of anxiety and panic, $2^{\text {nd }}$ ed. New York, NY: Guilford press.

Bartholow, B., Fabiani, M., Gratton, G., \& Bettencourt, A. (2001). A psychophysiological analysis of the processing time course of social expectancy violations. Psychological Science, 12, 197-204. 
Başoğlu, M. \& Mineka, S. (1992). The role of uncontrollable and unpredictable stress in posttraumatic stress responses in torture survivors. In M. Başoğlu (Ed.), Torture and its consequences: Current treatment approaches (pp. 182-225), New York, NY: Cambridge University Press.

Batson, C. D., Kennedy, C. L., Nord, L., Stocks, E. L., Fleming, D., Marzette, C. M., ... Zerger, T. (2007). Anger at unfairness: Is it moral outrage? European Journal of Social Psychology, 37, 1272-1285.

Baumeister, R. F., \& Leary, M. R. (1995). The need to belong: Desire for interpersonal attachments as a fundamental human motivation. Psychological Bulletin, 117, 497-529.

Beard, C., Weisberg, R. B., \& Keller, M. B. (2010). Health-related quality of life across the anxiety disorders: Findings from a sample of primary care patients. Journal of Anxiety Disorders, 24, 559-564.

Beck, A. T., \& Emery, G., \& Greenberg, R. L. (1985). Anxiety disorders and phobias: A cognitive perspective. New York, NY: Basic Books.

Beesdo, K., Hoyer, J., Jacobi, F., Low, N. C. P., Höfler, M., \& Wittchen, H.-U. (2009). Association between generalized anxiety levels and pain in a community sample: Evidence for diagnostic specificity. Journal of Anxiety Disorders, 23, 684-693.

Beesdo-Baum, K., Jenjahn, E., Höfler, M., Lueken, U., Becker, E. S., \& Hoyer, J. (2012). Avoidance, safety behavior, and reassurance seeking in generalized anxiety disorder. Depression and Anxiety, 29, 948-957.

Behar, E., Alcaine, O., Zuellig, A. R., \& Borkovec, T. D. (2003). Screening for generalized anxiety disorder using the Penn State Worry Questionnaire: A receiver operating 
characteristic analysis. Journal of Behavior Therapy and Experimental Psychiatry, 34, 25-43.

Behar, E., DiMarco, I. D., Hekler, E. B., Mohlman, J., \& Staples, A. M. (2009). Current theoretical models of generalized anxiety disorder (GAD): Conceptual review and treatment implications. Journal of Anxiety Disorders, 23, 1011-1023.

Belzer, K. D., D'Zurilla, T. J., \& Maydeu-Olivares, A. (2002). Social problem solving and trait anxiety as predictors of worry in a college student population. Personality and Individual Differences, 33, 573-585.

Bennett-Levy, J., Butler, G., Fennell, M., Hackmann, A., Mueller, M., \& Westbrook, D. (2004). Oxford guide to behavioural experiments in cognitive therapy. New York, NY: Oxford University Press.

Berenbaum, H., Thompson, R. J., \& Bredemeier, K. (2007). Perceived threat: Exploring its association with worry and its hypothesized antecedents. Behaviour Research and Therapy, 45, 2473-2482.

Berenbaum, H., Thompson, R. J., \& Pomerantz, E. M. (2007). The relation between worrying and concerns: The importance of perceived probability and cost. Behaviour Research and Therapy, 45, 301-311.

Berger, C. R., \& Calabrese, R. J. (1975). Some explorations in initial interaction and beyond: Toward a developmental theory of interpersonal communication. Human Communication Research, 1, 99-112.

Berlyne, D. E. (1957). Uncertainty and conflict: A point of contact between information-theory and behavior-theory concepts. Psychological Review, 64, 329. 
Bjork, R. A., \& Bjork, E. L. (2006). Optimizing treatment and instruction: implications of a new theory of disuse. In L. G. Nilsson, \& N. Ohta (Eds.), Memory and society: Psychological perspectives (pp. 116-140). New York, NY: Psychology Press.

Birrell, J., Meares, K., Wilkinson, A., \& Freeston, M. (2011). Toward a definition of intolerance of uncertainty: A review of factor analytical studies of the intolerance of uncertainty scale. Clinical Psychology Review, 31, 1198-1208.

Blenkiron, P. (2005). Stories and analogies in cognitive behaviour therapy: A clinical review. Behavioural and Cognitive Psychotherapy, 33, 45-59.

Bolognesi, F., Baldwin, D. S., \& Ruini, C. (2014). Psychological interventions in the treatment of generalized anxiety disorder: A structured review. Journal of Psychopathology, 20, 111-126.

Borkovec, T. D. (1985). Worry: A potentially valuable concept. Behaviour Research and Therapy, 23, 481-482.

Borkovec, T. D. (1994). The nature, functions, and origins of worry. In G. C. L. Davey, \& F. Tallis (Eds.), Worrying: Perspectives on theory, assessment and treatment (pp. 5-33). Oxford, UK: John Wiley \& Sons.

Borkovec, T. D. (2002). Life in the future versus life in the present. Clinical Psychology: Science and Practice, 9, 76-80.

Borkovec, T. D., Alcaine, O., \& Behar, E. (2004). Avoidance theory of worry and generalized anxiety disorder. In R. G. Heimberg, C. L. Turk, \& D. S. Mennin (Eds.), Generalized anxiety disorder: Advances in research and practices (pp. 77-108). New York, NY: Guilford Press. 
Borkovec, T. D., \& Costello, E. (1993). Efficacy of applied relaxation and cognitive-behavioral therapy in the treatment of generalized anxiety disorder. Journal of Consulting and Clinical Psychology, 61, 611.

Borkovec, T. D., Hazlett-Stevens, H., \& Diaz, M. L. (1999). The role of positive beliefs about worry in generalized anxiety disorder and its treatment. Clinical Psychology \& Psychotherapy, 6, 126-138.

Borkovec, T. D., \& Inz, J. (1990). The nature of worry in generalized anxiety disorder: A predominance of thought activity. Behaviour Research and Therapy, 28, 153.

Borkovec, T. D., Ray, W. J., \& Stöber, J. (1998). Worry: A cognitive phenomenon intimately linked to affective, physiological, and interpersonal behavioral processes. Cognitive Therapy and Research, 22, 561-576.

Borkovec, T. D., Robinson, E., Pruzinsky, T., \& DePree, J. A. (1983). Preliminary exploration of worry: Some characteristics and processes. Behaviour Research and Therapy, 21, 9-16.

Borkovec, T. D., \& Roemer, L. (1995). Perceived functions of worry among generalized anxiety disorder subjects: Distraction from more emotionally distressing topics? Journal of behavior therapy and experimental psychiatry, 26, 25-30.

Boswell, J. F., Thompson-Hollands, J., Farchione, T. J., \& Barlow, D. H. (2013). Intolerance of uncertainty: A common factor in the treatment of emotional disorders. Journal of Clinical Psychology, 69, 630-645.

Botti, S. (2004). The psychological pleasure and pain of choosing: when people prefer choosing at the cost of subsequent outcome satisfaction. Journal of Personality and Social Psychology, 87, 312-326. 
Bouton, M. E. (1993). Context, time, and memory retrieval in the interference paradigms of Pavlovian learning. Psychological Bulletin, 114, 80-99.

Bower, G. H. (1981). Mood and memory. American Psychologist, 36, 129-148.

Boyd, R. L., Robinson, M. D., \& Fetterman, A. K. (2011). Miller (1944) revisited: Movement times in relation to approach and avoidance conflicts. Journal of Experimental Social Psychology, 47, 1192-1197.

Brashers, D. E., Neidig, J. L., \& Goldsmith, D. J. (2004). Social support and the managements of uncertainty for people living with HIV or AIDS. Health Communication, 16, 305-331.

Brashers, D. E., Neidig, J. L., Haas, S. M., \& Dobbs, L. K., Cardillo, L. W., Russell, J. A. (2000). Communication in the management of uncertainty: The case of persons living with HIV or AIDS. Communication Monographs, 67, 63-84.

Braun, V., \& Clarke, V. (2006). Using thematic analysis in psychology. Qualitative Research in Psychology, 3, 77-101.

Breckler, S. J. (1984). Empirical validation of affect, behavior, and cognition as distinct components of attitude. Journal of Personality and Social Psychology, 47, 1191-1205.

Brockner, J., Tyler, T. R., \& Cooper-Schneider, R. (1992). The influence of prior commitment to an institution on reactions to perceived unfairness: The higher they are, the harder they fall. Administrative Science Quarterly, 37, 241-261.

Brockner, J., \& Wiesenfeld, B. M. (1996). An integrative framework for explaining reactions to decisions: interactive effects of outcomes and procedures. Psychological Bulletin, 120, 189-208.

Broman-Fulks, J. J., Hill, R. W., \& Green, B. A. (2008). Is perfectionism categorical or dimensional? A taxometric analysis. Journal of Personality Assessment, 90, 481-490. 
Brosschot, J. F., Gerin, W., \& Thayer, J. F. (2006). The perseverative cognition hypothesis: A review of worry, prolonged stress-related physiological activation, and health. Journal of Psychosomatic Research, 60, 113-124.

Brown, T. A., O’Leary, T. A., \& Barlow, D. H. (2001). Generalized anxiety disorder. In D. H. Barlow (Ed.), Clinical handbook of psychological disorders: A step-by-step treatment manual ( $3^{\text {rd }}$ ed., pp. 154-208). New York, NY: Guilford Press.

Bruce, S. E., Yonkers, K. A., Otto, M. W., Eisen, J. L., Weisberg, R. B., Pagano, M., ... Keller, M. B. (2005). Influence of psychiatric comorbidity on recovery and recurrence in generalized anxiety disorder, social phobia, and panic disorder: A 12-year prospective study. American Journal of Psychiatry, 162, 1179-1187.

Brun, W., \& Teigen, K. H. (1990). Prediction and postdiction preferences in guessing. Journal of Behavioral Decision Making, 3, 17-28.

Budner, S. (1962). Intolerance of ambiguity as a personality variable. Journal of Personality, 30, 29-50.

Buhr, K., \& Dugas, M. J. (2002). The Intolerance of Uncertainty Scale: Psychometric properties of the English version. Behaviour Research and Therapy, 40, 931-946.

Buhr, K., \& Dugas, M. J. (2006). Investigating the construct validity of intolerance of uncertainty and its unique relationship with worry. Journal of Anxiety Disorders, 20, 222236.

Burke, B. L., Arkowitz, H., \& Menchola, M. (2003). The efficacy of motivational interviewing: A meta-analysis of controlled clinical trials. Journal of Consulting and Clinical Psychology, 71, 843. 
Butler, G., \& Mathews, A. (1983). Cognitive processes in anxiety. Advances in Behaviour Research and Therapy, 5, 51-62.

Butler, G., \& Mathews, A. (1987). Anticipatory anxiety and risk perception. Cognitive Therapy and Research, 11, 551-565.

Byrne, S. P., Hunt, C. J., \& Chang, B. P. I. (2015). Comparing the roles of ambiguity and unpredictability in intolerance of uncertainty. Behaviour Change, 32, 26-34.

Carleton, R. N. (2012). The intolerance of uncertainty construct in the context of anxiety disorders: Theoretical and practical perspectives. Expert Review of Neurotherapeutics, 12, 937-947.

Carleton, R. N., Gosselin, P., \& Asmundson, G. J. G. (2010). The intolerance of uncertainty index: Replication and extension with an English sample. Psychological Assessment, 22, 396-406.

Carleton, R. N., Mulvogue, M. K., Thibodeau, M. A., McCabe, R. E., Antony, M. M., \& Asmundson, G. J. G. (2012). Increasingly certain about uncertainty: Intolerance of uncertainty across anxiety and depression. Journal of Anxiety Disorders, 26, 468-479.

Carleton, R. N., Norton, M. A. P., \& Asmundson, G. J. G. (2007). Fearing the unknown: A short version of the Intolerance of Uncertainty Scale. Journal of Anxiety Disorders, 21, 105117.

Caudle, D. D., Senior, A. C., Wetherell, J. L., Rhoades, H. M., Beck, J. G., Kunik, M. E., ... \& Stanley, M. A. (2007). Cognitive errors, symptom severity, and response to cognitive behavior therapy in older adults with generalized anxiety disorder. American Journal of Geriatric Psychiatry, 15, 680-689. 
Chang, E. C., D’Zurilla, T. J., \& Sanna, L. J. (2004). Social problem solving: Theory, research, and training. Washington, DC: American Psychological Association.

Chow, C. C., \& Sarin, R. K. (2002). Known, unknown, and unknowable uncertainties. Theory and Decision, 52, 127-138.

Clark, D. A. (1999). Anxiety disorders: Why they persist and how to treat them. Behaviour research and therapy, 37, S5-S27.

Clark, D. A. (2004).Cognitive behavioral therapy for OCD. New York, NY: Guilford Press.

Clark, D. A., \& Beck, A. T. (1989). Cognitive theory and therapy of anxiety and depression. In P. C. Kendall \& D. Watson (Eds.), Anxiety and depression: Distinctive and overlapping features (pp. 379-411). San Diego, CA: Academic Press.

Clark, D. A., \& Beck, A. T. (2010). Cognitive therapy of anxiety disorders: Science and practice. New York, NY: Guilford Press.

Clark, D. A., \& Beck, A. T. (2010). Cognitive theory and therapy of anxiety and depression: Convergence with neurobiological findings. Trends in Cognitive Sciences, 14, 418-424.

Clark, D. A., \& Inozu, M. (2014). Unwanted intrusive thoughts: Cultural, contextual, covariational, and characterological determinants of diversity. Journal of ObsessiveCompulsive and Related Disorders, 3, 195-204.

Clark-Carter, D. (2004). Quantitative Psychological Research. New York, NY: Taylor \& Francis.

Coles, M. E., Turk, C. L., \& Heimberg, R. G. (2007). Memory bias for threat in generalized anxiety disorder: The potential importance of stimulus relevance. Cognitive Behaviour Therapy, 36, 65-73. 
Cooney, R., \& Lang, A. T. F. (2007). Taking uncertainty seriously: Adaptive governance and international trade. European Journal of International Law, 18, 523.

Corbin, R. M. (1980). The secretary problem as a model of choice. Journal of Mathematical Psychology, 21, 1-29.

Cosmides, L., \& Tooby, J. (1996). Are humans good intuitive statisticians after all? Rethinking some conclusions from the literature on judgment under uncertainty. Cognition, 58, 1-73.

Cougle, J. R. (2012). What makes a quality therapy? A consideration of parsimony, ease, and efficiency. Behavior Therapy, 43, 468-481.

Cougle, J. R., Goetz, A. R., Fitch, K. E., \& Hawkins, K. A. (2011). Termination of washing compulsions: A problem of internal reference criteria or 'not just right' experience? Journal of Anxiety Disorders, 25, 801-805.

Covin, R., Ouimet, A. J., Seeds, P. M., \& Dozois, D. J. A. (2008). A meta-analysis of CBT for pathological worry among clients with GAD. Journal of Anxiety Disorders, 22, 108-116.

Crabtree, B. F., \& Miller, W. L. (1999). Doing qualitative research. New York, NY: Sage.

Craske, M. G. (1999). Anxiety disorders: Psychological approaches to theory and treatment. New York, NY: Basic Books.

Craske, M. G., \& Barlow, D. H. (2006). Mastering of your anxiety and worry: Workbook (2nd ed). New York, NY: Oxford University Press.

Craske, M. G., Treanor, M., Conway, C. C., Zbozinek, T., \& Vervliet, B. (2014). Maximizing exposure therapy: An inhibitory learning approach. Behaviour Research and Therapy, 58, $10-23$. 
Cuijpers, P., Sijbrandij, M., Koole, S., Huibers, M., Berking, M., \& Andersson, G. (2014). Psychological treatment of generalized anxiety disorder: A meta-analysis. Clinical Psychology Review, 34, 130-140.

Cuijpers, P., van Straten, A., \& Warmerdam, L. (2007). Behavioral activation treatments of depression: A meta-analysis. Clinical Psychology Review, 27, 318-326.

Curley, S. P., Yates, J. F., \& Abrams, R. A. (1986). Psychological sources of ambiguity avoidance. Organizational Behavior and Human Decision Processes, 38, 230-256.

D’ Zurilla, T. J., \& Nezu, A. M. (2010). Problem-solving therapy. In K. S. Dobson (Ed.), The handbook of cognitive-behavioral therapies ( $3^{\text {rd }}$ ed., pp. 197-225). New York, NY: Guilford Press.

Dadds, M. R., Gaffney, L. R., Kenardy, J., Oei, T. P., \& Evans, L. (1993). An exploration of the relationship between expression of hostility and the anxiety disorders. Journal of Psychiatric Research, 27, 17-26.

Davey, G. C. (1994). Worrying, social problem-solving abilities, and social problem-solving confidence. Behaviour Research and Therapy, 32, 327-330.

Davey, G. C. L., Eldridge, F., Drost, J., \& MacDonald, B. A. (2007). What ends a worry bout? An analysis of changes in mood and stop rule use across the catastrophising interview task. Behaviour Research and Therapy, 45, 1231.

Davey, G. C., Hampton, J., Farrell, J., \& Davidson, S. (1992). Some characteristics of worrying: Evidence for worrying and anxiety as separate constructs. Personality and Individual Differences, 13, 133-147. 
Davey, G. C. L., \& Levy, S. (1998). Catastrophic worrying: Personal inadequacy and a perseverative iterative style as features of the catastrophizing process. Journal of Abnormal Psychology, 107, 576-586.

Davey, G. C. L., Startup, H. M., MacDonald, C., Jenkins, D., \& Patterson, K. (2005). The use of "as many as can" versus "feel like continuing" stop rules during worrying. Cognitive Therapy and Research, 29, 155-169.

Davey, G. C. L., Tallis, F., \& Capuzzo, N. (1996). Beliefs about the consequences of worrying. Cognitive Therapy and Research, 20, 499-520.

Deacon, B., Kemp, J. J., Dixon, L. J., Sy, J. T., Farrell, N. R., \& Zhang, A. R. (2013). Maximizing the efficacy of interoceptive exposure by optimizing inhibitory learning: a randomized controlled trial. Behaviour Research and Therapy, 51, 588-596.

Decker, M. L., Turk, C. L., Hess, B., \& Murray, C. E. (2008). Emotion regulation among individuals classified with and without generalized anxiety disorder. Journal of Anxiety Disorders, 22, 485-494.

Deschênes, S., Dugas, M., Fracalanza, K., \& Koerner, N. (2012). The role of anger in generalized anxiety disorder. Cognitive Behaviour Therapy, 41, 261-271.

Dhar, S. K., González-Vallejo, C., \& Soman, D. (1999). Modeling the effects of advertised price claims: tensile versus precise claims? Marketing Science, 18, 154-177.

Dimidjian, S., Hollon, S. D., Dobson, K. S., Schmaling, K. B., Kohlenberg, R. J., Addis, M. E., ... \& Jacobson, N. S. (2006). Randomized trial of behavioral activation, cognitive therapy, and antidepressant medication in the acute treatment of adults with major depression. Journal of Consulting and Clinical Psychology, 74, 658. 
Donegan, E., \& Dugas, M. J. (2012). Generalized anxiety disorder: A comparison of symptom change in adults receiving cognitive-behavioral therapy or applied relaxation. Journal of Consulting and Clinical Psychology, 80, 490-496.

Donohew, L., Tipton, L., \& Clarke, P. (1973). A conceptual model of information seeking, avoiding, and processing. In L. Donohew (Ed.), New models for mass communication research (pp. 307-352). Oxford, UK: Sage.

Dozois, D. J., \& Westra, H. A. (2005). Development of the Anxiety Change Expectancy Scale (ACES) and validation in college, community, and clinical samples. Behaviour Research and Therapy, 43, 1655-1672.

Dugas, M. J., Anderson, K. G., Deschenes, S. S., \& Donegan, E. (2010). Generalized anxiety disorder publications: Where do we stand a decade later? Journal of Anxiety Disorders, 24, 780-784.

Dugas, M. J., Brillon, P., Savard, P., Turcotte, J., Gaudet, A., Ladouceur, R., . . . Gervais, N. J. (2010). A randomized clinical trial of cognitive-behavioral therapy and applied relaxation for adults with generalized anxiety disorder. Behavior Therapy, 41, 46-58. doi:10.1016/j.beth.2008.12.004

Dugas, M. J., Buhr, K., \& Ladouceur, R. (2004). The role of intolerance of uncertainty in etiology and maintenance. In R. G. Heimberg, C. L. Turk, \& D. S. Mennin (Eds.), Generalized anxiety disorder: Advances in research and practice (pp. 142-163). New York, NY: Guilford Press.

Dugas, M. J., Freeston, M. H., Doucet, C., Provencher, M., \& Ladouceur, R. (1995, November). Intolerance of uncertainty and thought suppression in worry. Paper presented at the meeting of the Association for Advancement of Behavior Therapy, Washington, DC. 
Dugas, M. J., Freeston, M. H., \& Ladouceur, R. (1997). Intolerance of uncertainty and problem orientation in worry. Cognitive Therapy and Research, 21, 593-606.

Dugas, M. J., Freeston, M. H., Ladouceur, R., Rhéaume, J., Provencher, M., \& Boisvert, J. (1998). Worry themes in primary GAD, secondary GAD, and other anxiety disorders. Journal of Anxiety Disorders, 12, 253-261.

Dugas, M. J., Freeston, M. H., Provencher, M. D., Lachance, S., Ladouceur, R., \& Gosselin, P. (2001). The Worry and Anxiety Questionnaire: Validation in non-clinical and clinical samples. Journal De Thérapie Comportementale Et Cognitive, 11, 31-36.

Dugas, M. J., Gagnon, F., Ladouceur, R., \& Freeston, M. H. (1998). Generalized anxiety disorder: A preliminary test of a conceptual model. Behaviour Research and Therapy, 36, 215-226.

Dugas, M. J., Gosselin, P., \& Ladouceur, R. (2001). Intolerance of uncertainty and worry: Investigating specificity in a nonclinical sample. Cognitive Therapy and Research, 25, 551-558.

Dugas, M. J., Hedayati, M., Karavidas, A., Buhr, K., Francis, K., \& Phillips, N. A. (2005). Intolerance of uncertainty and information processing: Evidence of biased recall and interpretations. Cognitive Therapy and Research, 29, 57-70.

Dugas, M. J., \& Koerner, N. (2005). Cognitive-behavioral treatment for generalized anxiety disorder: Current status and future directions. Journal of Cognitive Psychotherapy, 19, 61-81.

Dugas, M. J., \& Ladouceur, R. (1998). Analysis and treatment of generalized anxiety disorder. In V. E. Caballo (Ed.), International handbook of cognitive and behavioural treatments for psychological disorders (pp. 197-225). Oxford, UK: Pergamon/Elsevier Science. 
Dugas, M. J., \& Ladouceur, R. (2000). Treatment of GAD: Targeting intolerance of uncertainty in two types of worry. Behavior Modification, 24, 635-657.

Dugas, M. J., Ladouceur, R., Léger, E., Freeston, M. H., Langolis, F., Provencher, M. D., \& Boisvert, J. (2003). Group cognitive-behavioral therapy for generalized anxiety disorder: Treatment outcome and long-term follow-up. Journal of Consulting and Clinical Psychology, 71, 821-825.

Dugas, M. J., Letarte, H., Rhéaume, J., Freeston, M. H., \& Ladouceur, R. (1995). Worry and problem solving: Evidence of a specific relationship. Cognitive Therapy and Research, 19, 109-120.

Dugas, M. J., \& Robichaud, M. (2007). Cognitive-behavioral treatment for generalized anxiety disorder: From science to practice. New York, NY: Routledge.

Dugas, M. J., Savard, P., Gaudet, A., Turcotte, J., Laugesen, N., Robichaud, M., .. . Koerner, N. (2007). Can the components of a cognitive model predict the severity of generalized anxiety disorder? Behavior Therapy, 38, 169-178.

East, M. P., \& Watts, F. N. (1994). Worry and the suppression of imagery. Behaviour Research and Therapy, 32, 851-855.

Einstein, D. A. (2014). Extension of the transdiagnostic model to focus on intolerance of uncertainty: A review of the literature and implications for treatment. Clinical Psychology: Science and Practice, 21, 280-300.

Eisen, J. L., Phillips, K. A., Coles, M. E., \& Rasmussen, S. A. (2004). Insight in obsessive compulsive disorder and body dysmorphic disorder. Comprehensive Psychiatry, 45, 1015.

Ellis, A. (1962). Reason and emotion in psychotherapy. Oxford, UK: Lyle Stuart. 
Ellsberg, D. (1961). Risk, ambiguity, and the savage axioms. Quarterly Journal of Economics, 75, 643-669.

Eng, W., \& Heimberg, R. G. (2006). Interpersonal correlates of generalized anxiety disorder: Self versus other perception. Journal of Anxiety Disorders, 20, 380 -387.

Epstein, L. (1999) A definition of uncertainty aversion. Review of Economic Studies, 66, 579608.

Erdem, M., Çelik, C., Yetkin, S., \& Özgen, F. (2008). Anger level and anger expression in generalized anxiety disorder. Anadolu Psikiyatri Dergisi, 9, 203-207.

Etner, J., Jeleva, M., \& Tallon, J. (2010). Decision theory under ambiguity. Journal of Economic Surveys, 24, 1-45.

Fergus, T. A., Valentiner, D. P., Wu, K. D., \& McGrath, P. B. (2015). Examining the symptomlevel specificity of negative problem orientation in a clinical sample. Cognitive Behaviour Therapy, 44, 153-161.

Field, A. P. (2009). Discovering statistics using SPSS, $3^{\text {rd }}$ edition. London, UK: Sage.

Fisher, P. L., \& Wells, A. (2005). Experimental modification of beliefs in obsessive-compulsive disorder: A test of the metacognitive model. Behaviour Research and Therapy, 43, 821829.

Fleiss, L., Levin, B., \& Paik, M. C. (1981). The measurement of interrater agreement. In J. L. Fleiss, B. Levin, \& M. C. Paik (Eds.), In Statistical methods for rates and proportions (pp. 598-626). New York, NY: John Wiley \& Sons.

Flick, U. (2009). An introduction to qualitative research (4th Ed.). Thousand Oaks, CA: Sage. 
Foa, E. B., Huppert, J. D., \& Cahill, S. P. (2006). Emotional Processing Theory: An Update. In B. O. Rothbaum (Ed.). Pathological anxiety: Emotional processing in etiology and treatment (pp. 3-24). New York, NY: Guilford Press.

Foa, E. B., \& McNally, R. J. (1996). Mechanisms of change in exposure therapy. In M. Rapee (Ed.), Current controversies in the anxiety disorders (pp. 329-343). New York, NY: Guilford Press.

Foa, E. B., Zinbarg, R., \& Rothbaum, B. O. (1992). Uncontrollability and unpredictability in post-traumatic stress disorder: an animal model. Psychological Bulletin, 112, 218-238.

Forsyth, J. P., \& McNeil, D. W. (2002). Mastery of your anxiety and worry: A multimodal case study of the effectiveness of a manualized treatment for generalized anxiety disorder. Cognitive and Behavioral Practice, 9, 200-212.

Fox, C. R., \& Tversky, A. (1995). Ambiguity aversion and comparative ignorance. Quarterly Journal of Economics, 585-603.

Fracalanza, K., Koerner, N., \& Antony, M. M. (2014). Testing a procedural variant of written imaginal exposure for generalized anxiety disorder. Journal of Anxiety Disorders, 28, 559-569.

Fracalanza, K., Koerner, N., Deschênes, S. S., \& Dugas, M. J. (2014). Intolerance of uncertainty mediates the relation between generalized anxiety disorder symptoms and anger. Cognitive Behaviour Therapy, 43, 122-132.

Frances, A. J., \& Widiger, T. (2012). Psychiatric diagnosis: Lessons from the DSM-IV past and cautions for the DSM-5 future. Annual Review of Clinical Psychology, 8, 109-130. 
Frank, G. K. W., Roblek, T., Shott, M. E., Jappe, L. M., Rollin, M. D. H., Hagman, J. O., \& Pryor, T. (2012). Heightened fear of uncertainty in anorexia and bulimia nervosa. International Journal of Eating Disorders, 45, 227-232.

Freeston, M. H., Rhéaume, J., Letarte, H., \& Dugas, M. J. (1994). Why do people worry? Personality and Individual Differences, 17, 791-802.

Fresco, D. M., Mennin, D. S., Heimberg, R. G., \& Turk, C. L. (2003). Using the Penn State Worry Questionnaire to identify individuals with generalized anxiety disorder: A receiver operating characteristic analysis. Journal of Behavior Therapy and Experimental Psychiatry, 34, 283-291.

Furnham, A., \& Ribchester, T. (1995). Tolerance of ambiguity: A review of the concept, its measurement and applications. Current Psychology: A Journal for Diverse Perspectives on Diverse Psychological Issues, 14, 179-199.

Geer, J. H., Davison, G. C., \& Gatchel, R. J. (1970). Reduction of stress in humans through nonveridical perceived control of aversive stimulation. Journal of Personality and Social Psychology, 16, 731-738.

Geller, J., \& Dunn, E. C. (2011). Integrating motivational interviewing and cognitive behavioral therapy in the treatment of eating disorders: Tailoring interventions to patient readiness for change. Cognitive and Behavioral Practice, 18, 5-15.

Gentes, E. L., \& Ruscio, A. M. (2011). A meta-analysis of the relation of intolerance of uncertainty to symptoms of generalized anxiety disorder, major depressive disorder, and obsessive-compulsive disorder. Clinical Psychology Review, 31, 923-923-933. 
Germain, A., Shear, M. K., Hall, M., \& Buysse, D. J. (2007). Effects of a brief behavioral treatment for PTSD-related sleep disturbances: A pilot study. Behaviour Research and Therapy, 45, 627-632.

Glanzer, M. (1958). Curiosity, exploratory drive, and stimulus satiation. Psychological Bulletin, 55, 302-315.

Goldman, N., Dugas, M. J., Sexton, K. A., \& Gervais, N. J. (2007). The Impact of Written Exposure on Worry A Preliminary Investigation. Behavior Modification, 31, 512-538.

Goldsmith, K., \& Amir, O. (2010). Can uncertainty improve promotions? Journal of Marketing Research, 47, 1070-1077.

Gosselin, P., Ladouceur, R., Evers, A., Laverdière, A., Routhier, S., \& Tremblay-Picard, M. (2008). Evaluation of intolerance of uncertainty: Development and validation of a new self-report measure. Journal of Anxiety Disorders, 22, 1427-1439.

Gosselin, P., Ladouceur, R., Morin, C. M., Dugas, M. J., \& Baillargeon, L. (2006). Benzodiazepine discontinuation among adults with GAD: A randomized trial of cognitive-behavioral therapy. Journal of Consulting and Clinical Psychology, 74, 908919.

Gosselin, P., Langlois, F., Freeston, M. H., Ladouceur, R., Dugas, M. J., \& Pelletier, O. (2002). Le Questionnaire d'e'vitement cognitive (QEC): De'veloppement et validation aupre`s d'adultes et d'adolescents. Journal de The'rapie Comportementale et Cognitive, 12, 2437.

Grant, S. J., \& Tybout, A. M. (2008). The effect of temporal frame on information considered in new product evaluation: The role of uncertainty. Journal of Consumer Research, 34, 897913. 
Greenberg, J. (1999). Is the proposed role of excessive reassurance seeking in depression excessive? Psychological Inquiry, 10, 291-293.

Greenberger, D. \& Padesky, C. A. (1995). Mind over mood: Change how you feel by changing the way to think. New York, NY: Guilford Press.

Grillon, C., Baas, J. P., Lissek, S., Smith, K., \& Milstein, J. (2004). Anxious responses to predictable and unpredictable aversive events. Behavioral Neuroscience, 118, 916-924.

Grillon, C., Lissek, S., Rabin, S., McDowell, D., Dvir, S., \& Pine, D. S. (2008). Increased anxiety during anticipation of unpredictable but not predictable aversive stimuli as a psychophysiologic marker of panic disorder. American Journal of Psychiatry, 165, 898904.

Grillon, C., Pine, D. S., Lissek, S., Rabin, S., Bonne, O., \& Vythilingam, M. (2009). Increased anxiety during anticipation of unpredictable aversive stimuli in posttraumatic stress disorder but not in generalized anxiety disorder. Biological Psychiatry, 66, 47-53.

Grös, D. F., Antony, M. M., McCabe, R. E., \& Swinson, R. P. (2009). Frequency and severity of the symptoms of irritable bowel syndrome across the anxiety disorders and depression. Journal of Anxiety Disorders, 23, 290-296.

Grös, D. F., Antony, M. M., Simms, L. J., \& McCabe, R. E. (2007). Psychometric properties of the State-Trait Inventory for Cognitive and Somatic Anxiety (STICSA): Comparison to the State-Trait Anxiety Inventory (STAI). Psychological Assessment, 19, 369-381.

Grupe, D. W., \& Nitschke, J. B. (2013). Uncertainty and anticipation in anxiety: An integrated neurobiological and psychological perspective. Nature Reviews Neuroscience, 14, 488501. 
Guest, G., Bunce, A., Johnson, L. (2006). How many interviews are enough? An experiment with data saturation and variability. Field Methods, 18, 59-82.

Haeffel, G. J., Voelz, Z. R., \& Joiner, T. E. (2007). Vulnerability to depressive symptoms: Clarifying the role of excessive reassurance seeking and perceived social support in an interpersonal model of depression. Cognition and Emotion, 21, 681-688.

Halevy, Y. (2007). Ellsberg revisited: An experimental study. Econometrica, 75, 503-536.

Hancock, G. R., \& Klockars, A. J. (1996). The quest for $\alpha$ : Developments in multiple comparison procedures in the quarter century since Games (1971). Review of Educational Research, 66, 269-306.

Hanrahan, F., Field, A. P., Jones, F. W., \& Davey, G. C. L. (2013). A meta-analysis of cognitive therapy for worry in generalized anxiety disorder. Clinical Psychology Review, 33, 120132.

Hazlett-Stevens, H. (2008). Psychological approaches to generalized anxiety disorder: A clinician's guide to assessment and treatment. New York, NY: Springer.

Hazlett-Stevens, H., \& Craske, M. G. (2003). The catastrophizing worry process in generalized anxiety disorder: A preliminary investigation of an analog population. Behavioural and Cognitive Psychotherapy, 31, 387-401.

Hazlett-Stevens, H., Ullman, J. B., \& Craske, M. G. (2004). Factor structure of the Penn State Worry Questionnaire: Examination of a method factor. Assessment, 11, 361-370.

Hebert, E. A., Dugas, M. J., Geninet, I., Turcotte, J., Savard, P., \& Dao, T.-V. (2014, July). Behavioural experiments in the treatment of intolerance of uncertainty. Paper presented at the meeting of the British Association for Behavioural and Cognitive Psychotherapies, Birmingham, UK. 
Heerey, E. A., \& Kring, A. M. (2007). Interpersonal consequences of social anxiety. Journal of Abnormal Psychology, 116, 125-134.

Hendry, D. P. (1969). Reinforcing value of information: Fixed-ratio schedules. Conditioned Reinforcement, 1, 300-341.

Henning, E. R., Turk, C. L., Mennin, D. S., Fresco, D. M., \& Heimberg, R. G. (2007). Impairment and quality of life in individuals with generalized anxiety disorder. Depression and Anxiety, 24, 342-349.

Herry, C., Bach, D. R., Esposito, F., Di Salle, F., Perrig, W. J., Scheffler, K., ... \& Seifritz, E. (2007). Processing of temporal unpredictability in human and animal amygdala. Journal of Neuroscience, 27, 5958-5966.

Heyman, B., Henriksen, M., \& Maughan, K. (1998). Probabilities and health risks: A qualitative approach. Social Science and Medicine, 47, 1295-1306.

Hinds, A. L., Woody, E. Z., Ameringen, M. V., Schmidt, L. A., \& Szechtman, H. (2012). When too much is not enough: Obsessive-compulsive disorder as a pathology of stopping, rather than starting. PLoS One, 7, 1-9.

Hoffart, A., Borge, F. M., Sexton, H., \& Clark, D. M. (2009). Change processes in residential cognitive and interpersonal psychotherapy for social phobia: A process-outcome study. Behavior Therapy, 40, 10-22.

Hoffman, D. L., Dukes, E. M., \& Wittchen, H. (2008). Human and economic burden of generalized anxiety disorder. Depression and Anxiety, 25, 72-90.

Hofmann, S. G. (2008). Cognitive processes during fear acquisition and extinction in animals and humans: Implications for exposure therapy of anxiety disorders. Clinical Psychology Review, 28, 199-210. 
Hofmann, S. G., Moscovitch, D. A., Litz, B. T., Kim, H. J., Davis, L. L., \& Pizzagalli, D. A. (2005). The worried mind: Autonomic and prefrontal activation during worrying. Emotion, 5, 464-475.

Holaway, R. M., Heimberg, R. G., \& Coles, M. E. (2006). A comparison of intolerance of uncertainty in analogue obsessive-compulsive disorder and generalized anxiety disorder. Journal of Anxiety Disorders, 20, 158-174.

Holowka, D. W., Dugas, M. J., Francis, K., \& Laugesen, N. (2000, November). Measuring beliefs about worry: A psychometric evaluation of the Why Worry-II Questionnaire. Poster presented at the meeting of the Association for the Advancement of Behavior Therapy, New Orleans, LA.

Horan, S. M., Chory, R. M., \& Goodboy, A. K. (2010). Understanding students' classroom justice experiences and responses. Communication Education, 59, 453-474.

Howell, W. C. (1971). Uncertainty from internal and external sources: A clear case of overconfidence. Journal of Experimental Psychology, 89, 240-243.

Howell, W. C., \& Burnett, S. A. (1978). Uncertainty measurement: A cognitive taxonomy. Organizational Behavior and Human Performance, 22, 45-68.

Hsieh, H. F., \& Shannon, S. E. (2005). Three approaches to qualitative content analysis. Qualitative Health Research, 15, 1277-1288.

Humphreys, K. L., Marx, B. P., \& Lexington, J. M. (2008). Self-monitoring as a treatment vehicle. Cognitive behavior therapy: Applying empirically supported techniques in your practice (2nd ed.), pp. 478-485, Hoboken, NJ: John Wiley \& Sons. 
Hunt, C., Issakidis, C., \& Andrews, G. (2002). DSM-IV generalized anxiety disorder in the australian national survey of mental health and well-being. Psychological Medicine, 32, 649-659.

Inglis, I. R. (2000). Review: The central role of uncertainty reduction in determining behaviour. Behaviour, 137, 1567-1599.

Joiner, T. E., Jr., Alfano, M. S., \& Metalsky, G. I. (1992). When depression breeds contempt: Reassurance seeking, self-esteem, and rejection of depressed college students by their roommates. Journal of Abnormal Psychology, 101, 165-173.

Joiner, T. E., \& Metalsky, G. I. (2001). Excessive reassurance seeking: Delineating a risk factor involved in the development of depressive symptoms. Psychological Science, 12, 371378.

Joiner, T. E., Metalsky, G. I., Katz, J., \& Beach, S. R. (1999). Depression and excessive reassurance-seeking. Psychological Inquiry, 10, 269-278.

Jones, K. D. (2012). Dimensional and cross-cutting assessment in the DSM-5. Journal of Counseling and Development, 90, 481-487.

Joos, E., Vansteenwegen, D., Brunfaut, E., Bastiaens, T., Demyttenaere, K., Pieters, G., \& Hermans, D. (2012). The Penn State Worry Questionnaire-Past day: Development and validation of a measure assessing daily levels of worry. Journal of Psychopathology and Behavioral Assessment, 34, 35-47

Kahneman, D., \& Tversky, A. (1982). Variants of uncertainty. Cognition, 11, 143-157.

Kalma, A. (1986). Uncertainty reduction: A fundamental concept in understanding a number of psychological theories. Essays in Human Sociobiology, 2, 213-241. 
Kemeny, M. E. (2003). The psychobiology of stress. Current Directions in Psychological Science, 12, 124-129.

Kendall, P. C., \& Ingram, R. (1987). The future for cognitive assessment of anxiety: Let's get specific. In L. Michelson \& M. L. Ascher (Eds.), Anxiety and stress disorders: Cognitivebehavioral assessment and treatment (pp. 89-104). New York, NY: Guilford Press.

Kessler R. C., Berglund P. A., Demler O., Jin R., Merikangas, K. R., Walters E. E. (2005). Lifetime prevalence and age-of-onset distributions of DSM-IV disorders in the National Comorbidity Survey Replication (NCS-R). Archives of General Psychiatry, 62, 593-602.

Khawaja, N. G., McMahon, J., \& Strodl, E. (2011). Intolerance of uncertainty and meta-worry: Relative importance in discriminating GAD. Behaviour Change, 28, 181-194.

Khawaja, N. G., \& Yu, L. N. H. (2010). A comparison of the 27-item and 12-item Intolerance of Uncertainty Scales. Clinical Psychologist, 14, 97-106.

Kim, H., Lundh, L., \& Harvey, A. (2002). The enhancement of video feedback by cognitive preparation in the treatment of social anxiety: A single-session experiment. Journal of Behavior Therapy and Experimental Psychiatry, 33, 19-37.

Knobloch, L. K., \& Solomon, D. H. (1999). Measuring the sources and content of relational uncertainty. Communication Studies, 50, 261-278.

Koerner, N., Antony, M. M., Young, L., \& McCabe, R. E. (2013). Changes in beliefs about the social competence of self and others following group cognitive-behavioral treatment. Cognitive Therapy and Research, 37, 256-265.

Koerner, N., \& Dugas, M. J. (2008). An investigation of appraisals in individuals vulnerable to excessive worry: The role of intolerance of uncertainty. Cognitive Therapy and Research, 32, 619-638. 
Koerner, N., Tallon, K., \& Kusec, A. (2015). Maladaptive core beliefs and their relation to generalized anxiety disorder. Manuscript submitted for publication.

Korotitsch, W. J., \& Nelson-Gray, R. (1999). An overview of self-monitoring research in assessment and treatment. Psychological Assessment, 11, 415-425.

Kramer, M. W. (1999). Motivation to reduce uncertainty. Management Communication Quarterly, 13, 305-316.

Kraus, S. J. (1995). Attitudes and the prediction of behavior: A meta-analysis of the empirical literature. Personality and Social Psychology Bulletin, 21, 58-75.

Krohne. H. W. (1993). Vigilance and cognitive avoidance as concepts in coping research. In H. W. Krohne (Ed.), Attention and avoidance strategies in coping with aversiveness (pp. 1951). Seattle, WA: Hogrefe \& Huber.

LaPiere, R. T. (1934). Attitudes versus actions. Social Forces, 13, 230-237.

Lazarus, R. S., \& Folkman, S. (1987). Transactional theory and research on emotions and coping. European Journal of personality, 1, 141-169.

Lecrubier, Y., Sheehan, D. V., Weiller, E., Amorim, P., Bonora, I., Sheehan, K. H., ... \& Dunbar, G. C. (1997). The Mini International Neuropsychiatric Interview (MINI). A short diagnostic structured interview: Reliability and validity according to the CIDI. European Psychiatry, 12, 224-231.

Lemay, E. P., \& Cannon, K. T. (2012). Dysphoric reassurance seeking breeds contempt: Experimental evidence. Journal of Social and Clinical Psychology, 31, 1023-1050.

Linehan, M. M. (2015). DBT skills training manual, second edition. New York, NY: Guilford Press. 
Llera, S. J., \& Newman, M. G. (2010). Effects of worry on physiological and subjective reactivity to emotional stimuli in generalized anxiety disorder and nonanxious control participants. Emotion, 10, 640-650.

Llera, S. J., \& Newman, M. G. (2014). Rethinking the role of worry in generalized anxiety disorder: Evidence supporting a model of emotional contrast avoidance. Behavior Therapy, 45, 283-299.

Lohr, J. M., Olatunji, B. O., \& Sawchuk, C. N. (2007). A functional analysis of danger and safety signals in anxiety disorders. Clinical Psychology Review, 27, 114-126.

Luhmann, C. C., Ishida, K., \& Hajcak, G. (2011). Intolerance of uncertainty and decisions about delayed, probabilistic rewards. Behavior Therapy, 42, 378-386.

Lynch, T. R., Trost, W. T., Salsman, N., \& Linehan, M. M. (2007). Dialectical behavior therapy for borderline personality disorder. Annual Review of Clinical Psychology, 3, 181-205.

Kujanpää, T., Ylisaukko-oja, T., Jokelainen, J., Linna, M., \& Timonen, M. (2014). Comparative cost analysis of generalized anxiety disorder and major depressive disorder patients in secondary care from a national hospital registry in Finland. Nordic Journal of Psychiatry, 68, 306-310.

Kupor, D. M., Tormala, Z. L., \& Norton, M. I. (2014). The allure of unknown outcomes: Exploring the role of uncertainty in the preference for potential. Journal of Experimental Social Psychology, 55, 210-216.

Kuzel, A. (1992). Sampling in qualitative inquiry. In B. Crabtree \& W. Miller (Eds.), Doing qualitative research (pp. 31-44). Thousand Oaks, CA: Sage.

Ladouceur, R., Blais, F., Freeston, M. H., \& Dugas, M. J. (1998). Problem solving and problem orientation in generalized anxiety disorder. Journal of Anxiety Disorders, 12, 139-152. 
Ladouceur, R., Dugas, M. J., Freeston, M. H., Léger, E., Gagnon, F., \& Thibodeau, N. (2000). Efficacy of a cognitive-behavioral treatment for generalized anxiety disorder: Evaluation in a controlled clinical trial. Journal of Consulting and Clinical Psychology, 68, 957-964.

Ladouceur, R., Dugas, M. J., Freeston, M. H., Rhéaume, J., Blais, F., Boisvert, J., . . Thibodeau, N. (1999). Specificity of generalized anxiety disorder symptoms and processes. Behavior Therapy, 30, 191-207.

Ladouceur, R., Freeston, M. H., Dugas, M. J., Rheaume, J., Gagnon, F., Thibodeau, N., et al. (1995, November). Specific association between generalized anxiety disorder and intolerance of uncertainty among anxiety disorder patients. Paper presented at the meeting of the Association for Advancement of Behavior Therapy, Washington, DC.

Ladouceur, R., Gosselin, P., \& Dugas, M. J. (2000). Experimental manipulation of intolerance of uncertainty: A study of a theoretical model of worry. Behaviour Research and Therapy, 38, 933-941.

Ladouceur, R., Léger, É., Dugas, M., \& Freeston, M. H. (2004). Cognitive-behavioral treatment of generalized anxiety disorder (GAD) for older adults. International Psychogeriatrics, 16, 195-207.

Ladouceur, R., Talbot, F., \& Dugas, M. J. (1997). Behavioral expressions of intolerance of uncertainty in worry. Behavior Modification, 21, 355-371.

Leahy, R. L. (2005). Panic, agoraphobia, and generalized anxiety. In N. Kazantzis, F. P. Deane, K. R. Ronan \& L. L'Abate (Eds.), Using homework assignments in cognitive behavior therapy (pp. 193-218). New York, NY: Routledge/Taylor \& Francis.

Leahy, R. L. (2006). The worry cure: Seven steps to stop worry from stopping you. New York, NY: Crown Publishing Group. 
Lee, J. K., Orsillo, S. M., Roemer, L., \& Allen, L. B. (2010). Distress and avoidance in generalized anxiety disorder: Exploring the relationships with intolerance of uncertainty and worry. Cognitive Behaviour Therapy, 39, 126-136.

Lee, Y. H., \& Qiu, C. (2009). When uncertainty brings pleasure: The role of prospect imageability and mental imagery. Journal of Consumer Research, 36, 624-633.

Lewin, K. (1935). A dynamic theory of personality. New York, NY: McGraw Hill.

Lieberman, D. A., Cathro, J. S., Nichol, K., \& Watson, E. (1997). The role of S- in human observing behavior: Bad news is sometimes better than no news. Learning and Motivation, 28, 20-42.

Lipshitz, R., \& Strauss, O. (1997). Coping with uncertainty: A naturalistic decision-making analysis. Organizational Behavior and Human Decision Processes, 69, 149-163.

Loewenstein, G. F. (1994). The psychology of curiosity: A review and reinterpretation. Psychological Bulletin, 116, 75-98.

Loewenstein, G. F., Weber, E. U., Hsee, C. K., \& Welch, N. (2001). Risk as feelings. Psychological Bulletin, 127, 267-286.

Lyonfields, J. D., Borkovec, T. D., \& Thayer, J. F. (1995). Vagal tone in generalized anxiety disorder and the effects of aversive imagery and worrisome thinking. Behavior Therapy, 26, 457-466.

Maas. J. M., \& Hox, J. J. (2004). The influence of violations of assumptions on multilevel parameter estimates and their standard errors. Computational Statistics \& Data Analysis, 46, 427-440.

MacDonald, B., \& Davey, G. C. L. (2005). Inflated responsibility and perseverative checking: The effect of negative mood. Journal of Abnormal Psychology, 114, 176-182. 
MacLeod, A. K., Williams, J. M., \& Bekerian, D. A. (1991). Worry is reasonable: The role of explanations in pessimism about future personal events. Journal of Abnormal Psychology, 100, 478-486.

Maheswaran, D., \& Chaiken, S. (1991). Promoting systematic processing in low-motivation settings: Effect of incongruent information on processing and judgment. Journal of Personality and Social Psychology, 61, 13-25.

Mahoney, A. E. J., \& McEvoy, P. M. (2012). A transdiagnostic examination of intolerance of uncertainty across anxiety and depressive disorders. Cognitive Behaviour Therapy, 41, 212-222.

Marcus, M., Westra, H., Angus, L., \& Kertes, A. (2011). Client experiences of motivational interviewing for generalized anxiety disorder: A qualitative analysis. Psychotherapy Research, 21, 447-461.

Martens, E. J., de Jonge, P., Na, B., Cohen, B. E., Lett, H., \& Whooley, M. A. (2010). Scared to death? Generalized anxiety disorder and cardiovascular events in patients with stable coronary heart disease: The heart and soul study. Archives of General Psychiatry, 67, 750-758.

Mataix-Cols, D., do Rosario-Campos, M. C., \& Leckman, J. F. (2005). A multidimensional model of obsessive-compulsive disorder. American Journal of Psychiatry, 162, 228-238.

Matt, G. E., Vázquez, C., \& Campbell, W. K. (1992). Mood-congruent recall of affectively toned stimuli: A meta-analytic review. Clinical Psychology Review, 12, 227-255.

Mathews, A. (1990). Why worry? The cognitive function of anxiety. Behaviour Research and Therapy, 28, 455-468. 
McEvoy, P. M., \& Mahoney, A. E. (2011). Achieving certainty about the structure of intolerance of uncertainty in a treatment-seeking sample with anxiety and depression. Journal of Anxiety Disorders, 25, 112-122.

McEvoy, P. M., \& Mahoney, A. E. (2012). To be sure, to be sure: Intolerance of uncertainty mediates symptoms of various anxiety disorders and depression. Behavior Therapy, 43, 533-545.

McManus, F., Clark, D. M., Grey, N., Wild, J., Hirsch, C., Fennell, M., . . Manley, J. (2009). A demonstration of the efficacy of two of the components of cognitive therapy for social phobia. Journal of Anxiety Disorders, 23, 496-503.

McManus, F., Van Doorn, K., \& Yiend, J. (2012). Examining the effects of thought records and behavioral experiments in instigating belief change. Journal of Behavior Therapy and Experimental Psychiatry, 43, 540-547.

McMillan, D., \& Lee, R. (2010). A systematic review of behavioral experiments vs. exposure alone in the treatment of anxiety disorders: A case of exposure while wearing the emperor's new clothes? Clinical Psychology Review, 30, 467-478.

McNaughton, N. (2011). Fear, anxiety and their disorders: past, present and future neural theories. Psychology \& Neuroscience, 4, 173-181.

Meeten, F., Dash, S. R., Scarlet, A. L. S., \& Davey, G. C. L. (2012). Investigating the effect of intolerance of uncertainty on catastrophic worrying and mood. Behaviour Research and Therapy, 50, 690-698.

Mennin, D. S., Holaway, R. M., Fresco, D. M., Moore, M. T., \& Heimberg, R. G. (2007). Delineating components of emotion and its dysregulation in anxiety and mood psychopathology. Behavior Therapy, 38, 284-302. 
Mennin, D. S., Turk, C. L., Heimberg, R. G., \& Carmin, C. (2004). Regulation of emotion in generalized anxiety disorder. In M. A. Reinecke and D. A. Clark (Eds.), Cognitive therapy over the lifespan: Theory, research, and practice (pp. 60-89). Hoboken, NJ: John Wiley and Sons.

Metzger, R. L., Miller, M. L., Cohen, M., Sofka, M., \& Borkovec, T. D. (1990). Worry changes decision making: The effect of negative thoughts on cognitive processing. Journal of Clinical Psychology, 46, 78-88.

Meyer, T. J., Miller, M. L., Metzger, R. L., \& Borkovec, T. D. (1990). Development and validation of the Penn State Worry Questionnaire. Behaviour Research and Therapy, 28, 487-495. doi: 10.1016/0005-7967(90)90135-6

Miceli, M., \& Castelfranchi, C. (2005). Anxiety as an "epistemic" emotion: An uncertainty theory of anxiety. Anxiety, Stress \& Coping, 18, 291-319.

Michl, L. C., McLaughlin, K. A., Shepherd, K., \& Nolen-Hoeksema, S. (2013). Rumination as a mechanism linking stressful life events to symptoms of depression and anxiety: Longitudinal evidence in early adolescents and adults. Journal of Abnormal Psychology, 122, 339-352.

Miller, L. E. (2012). Sources of uncertainty in cancer survivorship. Journal of Cancer Survivorship, 6, 431-440. doi: http://dx.doi.org/10.1007/s11764-012-0229-7

Miller, N. E. (1944). Experimental studies of conflict. In J. M. Hunt (Ed.), Personality and the behaviour disorders. (pp. 431-465). New York, NY: Ronald.

Miller, S. M. (1987). Monitoring and blunting: Validation of a questionnaire to assess styles of information seeking under threat. Journal of Personality and Social Psychology, 52, 345353. 
Mineka, S. (1985). The frightful complexity of the origins of fears. In F. R. Brush \& J. B. Overmier (Eds.), Affect, conditioning, and cognition, (pp. 55-73). Hillsdale, NJ: Erlbaum.

Mineka, S., \& Hendersen, R. W. (1985). Controllability and predictability in acquired motivation. Annual Review of Psychology, 36, 495-529.

Mineka, S., \& Zinbarg, R. (2006). A contemporary learning theory perspective on the etiology of anxiety disorders: It's not what you thought it was. American Psychologist, 61, 10-26.

Miranda, R., \& Mennin, D. S. (2007). Depression, generalized anxiety disorder, and certainty in pessimistic predictions about the future. Cognitive Therapy and Research, 31, 71-82.

Mischel, M. H. (1990). Reconceptualization of the uncertainty in illness theory. Journal of Nursing Scholarship, 22, 256-262.

Mitte, K. (2007). Anxiety and risky decision-making: The role of subjective probability and subjective costs of negative events. Personality and Individual Differences, 43, 243-253.

Montesano, A., \& Giovannoni, F. (1996). Uncertainty aversion and aversion to increasing uncertainty. Theory and Decision, 41, 133-148.

Moore, M. T., Anderson, N. L., Barnes, J. M., Haigh, E. A. P., \& Fresco, D. M. (2014). Using the GAD-Q-IV to identify generalized anxiety disorder in psychiatric treatment seeking and primary care medical samples. Journal of Anxiety Disorders, 28, 25-30.

Moore, R., Brødsgaard, I., \& Rosenberg, N. (2004). The contribution of embarrassment to phobic dental anxiety: A qualitative research study. BMC Psychiatry, 4, 1-11.

Morrison, E. W., \& Robinson, S. L. (1997). When employees feel betrayed: A model of how psychological contract violation develops. Academy of Management Review, 22, 226-256.

Moscovitch, D. A., Antony, M. M., and Swinson, R. P. (2009). Exposure-based treatments for anxiety disorders: Theory and process. In M. M. Antony and M. B. Stein (Eds.), Oxford 
handbook of anxiety and related disorders (pp. 461-475). New York, NY: Oxford University Press.

Muris, P. E. H. M., Van Zuuren, F. J., De Jong, P. J., De Beurs, E., \& Hanewald, G. J. F. P. (1994). Monitoring and blunting coping styles: The Miller Behavioural Style Scale and its correlates, and the development of an alternative questionnaire. Personality and Individual Differences, 17, 9-19.

Narrow, W. E., \& Kuhl, E. A. (2011). Dimensional approaches to psychiatric diagnosis in DSM5. Journal of Mental Health Policy and Economics, 14, 197-200.

Nelson, E. A., Deacon, B. J., Lickel, J. J., \& Sy, J. T. (2010). Targeting the probability versus cost of feared outcomes in public speaking anxiety. Behaviour Research and Therapy, 48, 282-289.

Newman, M. G., \& Fisher, A. J. (2010). Expectancy/credibility change as a mediator of cognitive behavioral therapy for generalized anxiety disorder: Mechanism of action or proxy for symptom change? International Journal of Cognitive Therapy, 3, 245-261.

Newman, M. G., \& Llera, S. J. (2011). A novel theory of experiential avoidance in generalized anxiety disorder: A review and synthesis of research supporting a contrast avoidance model of worry. Clinical Psychology Review, 31, 371-382.

Newman, M. G., Llera, S. J., Erickson, T. M., Przeworski, A., \& Castonguay, L. G. (2013). Worry and generalized anxiety disorder: A review and theoretical synthesis of evidence on nature, etiology, mechanisms, and treatment. Annual Review of Clinical Psychology, 9, 275-297.

Newman, M. G., Przeworski, A., Fisher, A. J., \& Borkovec, T. D. (2010). Diagnostic comorbidity in adults with generalized anxiety disorder: Impact of comorbidity on 
psychotherapy outcome and impact of psychotherapy on comorbid diagnoses. Behavior Therapy, 41, 59-72.

Newman, M. G., Zuellig, A. R., Kachin, K. E., Constantino, M. J., Przeworski, A., Erickson, T., \& Cashman-McGrath, L. (2002). Preliminary reliability and validity of the Generalized Anxiety Disorder Questionnaire-IV: A revised self-report diagnostic measure of generalized anxiety disorder. Behavior Therapy, 33, 215-233.

Norton, P. J., Barrera, T. L., Mathew, A. R., Chamberlain, L. D., Szafranski, D. D., Reddy, R., \& Smith, A. H. (2013). Effect of transdiagnostic CBT for anxiety disorders on comorbid diagnoses. Depression and Anxiety, 30, 168-173.

Norton, M. I., Frost, J. H., \& Ariely, D. (2007). Less is more: the lure of ambiguity, or why familiarity breeds contempt. Journal of Personality and Social Psychology, 92, 97-105.

Olatunji, B. O., Broman-Fulks, J., Bergman, S. M., Green, B. A., \& Zlomke, K. R. (2010). A taxometric investigation of the latent structure of worry: Dimensionality and associations with depression, anxiety, and stress. Behavior Therapy, 41, 212-228.

Olatunji, B. O., Cisler, J. M., Tolin, D. F. (2007). Quality of life in the anxiety disorders: A metaanalytic review. Clinical Psychology Review, 27, 572-581.

Orasanu, J., \& Connolly, T. (1993). The reinvention of decision making. In G. A. Klein, J. Orasanu, R. Calderwood \& C. E. Zsambok (Eds.), Linking expertise and naturalistic decision making (pp. 3-20). Westport, CT: Ablex Publishing.

Otto, M. W., \& Hofmann, S. G. (2009). Avoiding treatment failures in the anxiety disorders. New York, NY: Springer.

Ougrin, D. (2011). Efficacy of exposure versus cognitive therapy in anxiety disorders: Systematic review and meta-analysis. BMC Psychiatry, 11, 1-12. 
Panksepp, J. (1998). Affective neuroscience: The foundations of human and animal emotions. New York, NY: Oxford University Press.

Parrish, C. L., \& Radomsky, A. S. (2010). Why do people seek reassurance and check repeatedly? An investigation of factors involved in compulsive behavior in OCD and depression. Journal of Anxiety Disorders, 24, 211-222.

Pawluk, E. J., \& Koerner, N. (2013). A preliminary investigation of impulsivity in generalized anxiety disorder. Personality and Individual Differences, 54, 732-737.

Peasley-Miklus, C., \& Vrana, S. R. (2000). Effect of worrisome and relaxing thinking on fearful emotional processing. Behaviour Research and Therapy, 38, 129-144.

Perone, M., \& Kaminski, B. J. (1992). Conditioned reinforcement of human observing behavior by descriptive and arbitrary verbal stimuli. Journal of the Experimental Analysis of Behavior, 58, 557-575.

Peters, M. L., Godaert, G. L., Ballieux, R. E., van Vliet, M., Willemsen, J. J., Sweep, F. C., \& Heijnen, C. J. (1998). Cardiovascular and endocrine responses to experimental stress: Effects of mental effort and controllability. Psychoneuroendocrinology, 23, 1-17.

Pieper, S., Brosschot, J. F., van der Leeden, R., \& Thayer, J. F. (2010). Prolonged cardiac effects of momentary assessed stressful events and worry episodes. Psychosomatic Medicine, 72, $570-577$.

Potthoff, J. G., Holahan, C. J., \& Joiner, T. E. (1995). Reassurance seeking, stress generation, and depressive symptoms: an integrative model. Journal of Personality and Social Psychology, 68, 664-670. 
Price, M., \& Anderson, P. L. (2012). Outcome expectancy as a predictor of treatment response in cognitive behavioral therapy for public speaking fears within social anxiety disorder. Psychotherapy, 49, 173-179.

Price, D. D., Finniss, D. G., \& Benedetti, F. (2008). A comprehensive review of the placebo effect: Recent advances and current thought. Annual Review of Psychology, 59, 565-590.

Primakoff, L., Epstein, N., Covi, L. (1986). Homework compliance: An uncontrolled variable in cognitive therapy outcome research. Behavior Therapy, 17, 433-446.

Provencher, M. D., Freeston, M. H., Dugas, M. J., \& Ladouceur, R. (2000). Catastrophizing assessment of worry and threat schemata among worriers. Behavioural and Cognitive Psychotherapy, 28, 211-224.

Rachman, S. (1997). The evolution of cognitive behaviour therapy. In D. M. Clark, \& C. G. Fairburn (Eds.), Science and practice of cognitive behaviour therapy (pp. 3-26). New York, NY: Oxford University Press.

Rachman, S., \& de Silva, P. (1978). Abnormal and normal obsessions. Behaviour research and therapy, 16, 233-248.

Radloff, L. S. (1977). The CES-D scale: A self-report depression scale for research in the general population. Applied Psychological Measurement, 1, 385-401.

Radomsky, A. S., Rachman, S., Teachman, B. A., \& Freeman, W. S. (1998). Why do episodes of panic stop? Journal of Anxiety Disorders, 12, 263-270.

Raffa, S. D., White, K. S., \& Barlow, D. H. (2004). Feared consequences of panic attacks in panic disorder: A qualitative and quantitative analysis. Cognitive Behaviour Therapy, 33, 199-207. 
Raghunathan, R., \& Pham, M. T. (1999). All negative moods are not equal: Motivational influences of anxiety and sadness on decision making. Organizational Behavior and Human Decision Processes, 79, 56-77.

Rapee, R. M., Craske, M. G., Brown, T. A., \& Barlow, D. H. (1996). Measurement of perceived control over anxiety-related events. Behavior Therapy, 27, 279-293.

Raudenbush, S. W., Bryk, A. S., Cheong, Y. F., Congdon, R., \& du Toit, M. (2004). HLM 6: Hierarchical linear and nonlinear modeling. Lincolnwood, IL: SSI Scientific Software International.

Rausch, S. M., Gramling, S. E., \& Auerbach, S. M. (2006). Effects of a single session of largegroup meditation and progressive muscle relaxation training on stress reduction, reactivity, and recovery. International Journal of Stress Management, 13, 273-290.

Ree, M. J., French, D., MacLeod, C., \& Locke, V. (2008). Distinguishing cognitive and somatic dimensions of state and trait anxiety: Development and validation of the State-Trait Inventory for Cognitive and Somatic Anxiety (STICSA). Behavioural and Cognitive Psychotherapy, 36, 313-332.

Rees, C. S., McEvoy, P., \& Nathan, P. R. (2005). Relationship between homework completion and outcome in cognitive behaviour therapy. Cognitive Behaviour Therapy, 34, 242-247.

Regier, D. A. (2010). Diagnostic issues in depression and generalized anxiety disorder: Refining the research agenda for DSM-V. Arlington, VA: American Psychiatric Association.

Regier, D. A., Kuhl, E. A., \& Kupfer, D. J. (2013). The DSM-5: Classification and criteria changes. World Psychiatry, 12, 92-98.

Regier, D. A., Narrow, W. E., Kuhl, E. A., \& Kupfer, D. J. (2009). The conceptual development of DSM-V. American Journal of Psychiatry, 166, 645-650. 
Rescorla, R. A. (2006). Deepened extinction from compound stimulus presentation. Journal of Experimental Psychology: Animal Behavior Processes, 32, 135.

Rescorla, R. A. \& Wagner, A. R. (1972). A theory of Pavlovian conditioning: Variations in the effectiveness of reinforcement and nonreinforcement. In A. H. Black \& W. K. Prokasy (Eds.), Classical conditioning II: Current research and theory (pp. 64 -99). New York, NY: Appleton-Century-Crofts.

Robichaud, M. (2013). Cognitive behavior therapy targeting intolerance of uncertainty: Application to a clinical case of generalized anxiety disorder. Cognitive and Behavioral Practice, 20, 251-263.

Robichaud, M., \& Dugas, M. J. (2005). Negative problem orientation (part I): Psychometric properties of a new measure. Behaviour Research and Therapy, 43, 391-401.

Robinson, M. D., \& Gordon, K. H. (2011). Personality dynamics: Insights from the personality social cognitive literature. Journal of Personality Assessment, 93, 161-176.

Robinson, S. L., \& Rousseau, D. M. (1994). Violating the psychological contract: Not the exception but the norm. Journal of Organizational Behavior, 15, 245-259.

Roemer, L., Molina, S., \& Borkovec, T. D. (1997). An investigation of worry content among generally anxious individuals. Journal of Nervous and Mental Disease, 185, 314-319.

Roemer, L., \& Orsillo, S. M. (2007). An open trial of an acceptance-based behavior therapy for generalized anxiety disorder. Behavior Therapy, 38, 72-85.

Roemer, L., Orsillo, S. M., \& Salters-Pedneault, K. (2008). Efficacy of an acceptance-based behavior therapy for generalized anxiety disorder: Evaluation in a randomized controlled trial. Journal of Consulting and Clinical Psychology, 76, 1083-1089. 
Roemer, L., Salters, K., Raffa, S. D., \& Orsillo, S. M. (2005). Fear and avoidance of internal experiences in GAD: Preliminary tests of a conceptual model. Cognitive Therapy and Research, 29, 71-88.

Rolfe, G. (2006). Validity, trustworthiness and rigour: quality and the idea of qualitative research. Journal of Advanced Nursing, 53, 304-310.

Romera, I., Montejo, Á. L., Caballero, F., Caballero, L., Arbesú, J., Polavieja, P., . . . Gilaberte, I. (2011). Functional impairment related to painful physical symptoms in patients with generalized anxiety disorder with or without comorbid major depressive disorder: Post hoc analysis of a cross-sectional study. BMC Psychiatry, 11, 69-79.

Rosen, N. O., Ivanova, E., \& Knäuper, B. (2014). Differentiating intolerance of uncertainty from three related but distinct constructs. Anxiety, Stress \& Coping, 27, 55-73.

Rosen, N. O., \& Knäuper, B. (2009). A little uncertainty goes a long way: State and trait differences in uncertainty interact to increase information seeking but also increase worry. Health Communication, 24, 228-238.

Rosen, N. O., Knäuper, B., Di Dio, P., Morrison, E., Tabing, R., Feldstain, A., . . Rosberger, Z. (2010). The impact of intolerance of uncertainty on anxiety after receiving an informational intervention about HPV: A randomised controlled study. Psychology \& Health, 25, 651-668.

Rosen, N. O., Knäuper, B., \& Sammut, J. (2007). Do individual differences in intolerance of uncertainty affect health monitoring? Psychology and Health, 22, 413-430.

Ruscio, A. M., Borkovec, T. D., \& Ruscio, J. (2001). A taxometric investigation of the latent structure of worry. Journal of Abnormal Psychology, 110, 413-422. 
Rygh, J. L., \& Sanderson, W. C. (2004). Treating generalized anxiety disorder: Evidence-based strategies, tools, and techniques. New York, NY: Guilford Press.

Salkovskis, P. M. (1985). Obsessional-compulsive problems: A cognitive-behavioural analysis. Behaviour Research and Therapy, 23, 571-583.

Salkovskis, P. M. (1991). The importance of behaviour in the maintenance of anxiety and panic: A cognitive account. Behavioural Psychotherapy, 19, 6-19.

Salkovskis, P. M., Clark, D. M., Hackmann, A., Wells, A., \& Gelder, M. G. (1999). An experimental investigation of the role of safety-seeking behaviours in the maintenance of panic disorder with agoraphobia. Behaviour Research and Therapy, 37, 559-574.

Salkovskis, P. M., Hackmann, A., Wells, A., Gelder, M. G., \& Clark, D. M. (2007). Belief disconfirmation versus habituation approaches to situational exposure in panic disorder with agoraphobia: A pilot study. Behaviour Research and Therapy, 45, 877-885.

Schmidt, R. A., \& Bjork, R. A. (1992). New conceptualizations of practice: Common principles in three paradigms suggest new concepts for training. Psychological Science, 3, 207-217.

Schreier, M. (2012). Qualitative Content Analysis in Practice. New York, NY: Sage.

Schultz, W., Dayan, P., \& Montague, P. R. (1997). A neural substrate of prediction and reward. Science, 275, 1593-1599.

Selye, H. (1976). The stress concept. Canadian Medical Association Journal, 115, 53-56.

Sexton, K. A., \& Dugas, M. J. (2008). The Cognitive Avoidance Questionnaire: Validation of the English translation. Journal of Anxiety Disorders, 22, 355-370.

Sexton, K. A., \& Dugas, M. J. (2009). Defining distinct negative beliefs about uncertainty: Validating the factor structure of the intolerance of uncertainty scale. Psychological Assessment, 21, 176-186. 
Sheehan, D. V., Lecrubier, Y., Sheehan, K. H., Amorim, P., Janavs, J., Weiller, E., Hergueta, T., Baker, R., \& Dunbar, G. C. (1998). The Mini-International Neuropsychiatric Interview (M.I.N.I): The development and validation of a structured diagnostic psychiatric interview for DSM-IV and ICD-10. Journal of Clinical Psychiatry, 59, 22-33.

Shen, L., Fishbach, A., \& Hsee, C. K. (2014). The motivating-uncertainty effect: Uncertainty increases resource investment in the process of reward pursuit. Journal of Consumer Research, 41, 1301-1315.

Shirneshan, E., Bailey, J., Relyea, G., Franklin, B. E., Solomon, D. K., \& Brown, L. M. (2013). Incremental direct medical expenditures associated with anxiety disorders for the U.S. adult population: Evidence from the medical expenditure panel survey. Journal of Anxiety Disorders, 27, 720-727.

Sibrava, N. J., \& Borkovec, T. D. (2006). The cognitive avoidance theory of worry. In G. C. L. Davey \& A. Wells (Eds.), Worry and its psychological disorders: Theory, assessment and treatment (pp. 239-256). Chichester, UK: John Wiley and Sons.

Simmons, A. N., Flagan, T. M., Wittmann, M., Strigo, I. A., Matthews, S. C., Donovan, H., . . Paulus, M. P. (2013). The effects of temporal unpredictability in anticipation of negative events in combat veterans with PTSD. Journal of Affective Disorders, 146, 426-432.

Simmons, A., Strigo, I., Matthews, S. C., Paulus, M. P., \& Stein, M. B. (2006). Anticipation of aversive visual stimuli is associated with increased insula activation in anxiety-prone subjects. Biological Psychiatry, 60, 402-409.

Smith, C. A., \& Ellsworth, P. C. (1985). Patterns of cognitive appraisal in emotion. Journal of Personality and Social Psychology, 48, 813-838. 
Smith, C. A., \& Lazarus, R. S. (1993). Appraisal components, core relational themes, and the emotions. Cognition \& Emotion, 7, 233-269.

Sorrentino, R. M., \& Short, J. C. (1986). Uncertainty orientation, motivation, and cognition. In R. M. Sorrentino \& E.T. Higgins (Eds.), Handbook of motivation and cognition: Foundations of social behaviour (pp. 379-403). New York, NY: Guilford Press.

Stapinski, L. A., Abbott, M. J., \& Rapee, R. M. (2010). Evaluating the cognitive avoidance model of generalised anxiety disorder: Impact of worry on threat appraisal, perceived control and anxious arousal. Behaviour Research and Therapy, 48, 1032-1040.

Starr, L. R., \& Davila, J. (2008). Excessive reassurance seeking, depression, and interpersonal rejection: A meta-analytic review. Journal of Abnormal Psychology, 117, 762-775.

Starcevic, V., \& Berle, D. (2006). Cognitive specificity of anxiety disorders: A review of selected key constructs. Depression and Anxiety, 23, 51-61.

Starcevic, V., \& Portman, M. E. (2013). The status quo as a good outcome: How the DSM-5 diagnostic criteria for generalized anxiety disorder remained unchanged from the DSMIV criteria. Australian and New Zealand Journal of Psychiatry, 47, 995-997.

Startup, H. M., \& Davey, G. C. L. (2003). Inflated responsibility and the use of stop rules for catastrophic worrying. Behaviour Research and Therapy, 41, 495-503.

Startup, H. M., \& Erickson, T. M. (2006). The Penn State Worry Questionnaire (PSWQ). In G. C. L. Davey \& A. Wells (Eds.), Worry and its psychological disorders: Theory, assessment and treatment (pp. 101-119). Chichester, UK: John Wiley \& Sons.

Statistics Canada (2013). Canadian Community Health Survey - Mental Health User Guide. Ottawa: Statistics Canada. (accessed February 12, 2015). 
Steel, P. (2007). The nature of procrastination: A meta-analytic and theoretical review of quintessential self-regulatory failure. Psychological Bulletin, 133, 65-94.

Steketee, G., \& Chambless, D. L. (1992). Methodological issues in prediction of treatment outcome. Clinical Psychology Review, 12, 387-400.

Sternheim, L., Startup, H., \& Schmidt, U. (2011). An experimental exploration of behavioral and cognitive-emotional aspects of intolerance of uncertainty in eating disorder patients. Journal of Anxiety Disorders, 25, 806-812.

Stewart, R. E., \& Chambless, D. L. (2009). Cognitive-behavioral therapy for adult anxiety disorders in clinical practice: A meta-analysis of effectiveness studies. Journal of Consulting and Clinical Psychology, 77, 595-606.

Stöber, J. (1998). Worry, problem elaboration and suppression of imagery: The role of concreteness. Behaviour Research and Therapy, 36, 751-756.

Stöber, J., \& Bittencourt, J. (1998). Weekly assessment of worry: An adaptation of the Penn State Worry Questionnaire for monitoring changes during treatment. Behaviour Research and Therapy, 36, 645-656.

Stöber, J., \& Joormann, J. (2001). Worry, procrastination, and perfectionism: Differentiating amount of worry, pathological worry, anxiety, and depression. Cognitive Therapy and Research, 25, 49-60.

Swales, M. A. (2009). Dialectical behaviour therapy: Description, research and future directions. International Journal of Behavioral Consultation and Therapy, 5, 164-177.

Szechtman, H., \& Woody, E. (2004). Obsessive-compulsive disorder as a disturbance of security motivation. Psychological review, 111, 111-127. 
Tallis, F., Eysenck, M. H., \& Mathews, A. (1991). Elevated evidence requirements and worry. Personality and Individual Differences, 12, 21-27.

Tang, N. K. Y., Salkovskis, P. M., Hodges, A., Soong, E., Hanna, M. H., \& Hester, J. (2009). Chronic pain syndrome associated with health anxiety: A qualitative thematic comparison between pain patients with high and low health anxiety. British Journal of Clinical Psychology, 48, 1-20.

Taylor, C. T., \& Alden, L. E. (2008). Self-related and interpersonal judgment biases in social anxiety disorder: Changes during treatment and relationship to outcome. International Journal of Cognitive Therapy, 1, 125-137.

Teddlie, C., \& Tashakkori, A. (2009). Foundations of mixed methods research: Integrating quantitative and qualitative approaches in the social and behavioral sciences. Thousand Oaks, CA: Sage.

Teigen, K.H. (1994). Variants of subjective probabilities: Concepts, norms and biases. In G. Wright, G. \& P. Ayton (Eds.), Subjective probability (pp. 211 -238). Chichester, UK: John Wiley and Sons.

Thompson, J. D. (2003). Organizations in action: Social science bases of administrative theory. New Brunswick, NJ: Transaction Publishers.

Tice, D. M., \& Baumeister, R. F. (1997). Longitudinal study of procrastination, performance, stress, and health: The costs and benefits of dawdling. Psychological science, 454-458.

Tong, E. M. W., Tan, D. H., \& Tan, Y. L. (2013). Can implicit appraisal concepts produce emotion-specific effects? A focus on unfairness and anger. Consciousness and Cognition: An International Journal, 22, 449-460. 
Tormala, Z. L., Jia, J. S., \& Norton, M. I. (2012). The preference for potential. Journal of Personality and Social Psychology, 103, 567-583.

Turk, C. L., Heimberg, R. G., Luterek, J. A., Mennin, D. S., \& Fresco, D. M. (2005). Emotion dysregulation in generalized anxiety disorder: A comparison with social anxiety disorder. Cognitive Therapy and Research, 29, 89-106.

Van den Bos, K., \& Lind, E. A. (2002). Uncertainty management by means of fairness judgments. Advances in experimental social psychology, 34, 1-60.

Van den Bos, K. \& Lind, E. A. (2013). The social psychology of fairness and the regulation of personal uncertainty. In R. M. Arkin, K. C. Oleson, and P. J. Carroll (Eds.), Handbook of the uncertain self (pp. 122- 141). New York, NY: Psychology Press.

van der Heiden, C., Muris, P., \& van der Molen, H. T. (2012). Randomized controlled trial on the effectiveness of metacognitive therapy and intolerance-of-uncertainty therapy for generalized anxiety disorder. Behaviour Research and Therapy, 50, 100-109.

Van Zuuren, F. J., \& Wolfs, H. M. (1991). Styles of information seeking under threat: personal and situational aspects of monitoring and blunting. Personality and Individual Differences, 12, 141-149.

Vansteenwegen, D., Vervliet, B., Iberico, C., Baeyens, F., Van den Bergh, O., \& Hermans, D. (2007). The repeated confrontation with videotapes of spiders in multiple contexts attenuates renewal of fear in spider-anxious students. Behaviour Research and Therapy, 45, 1169-1179.

Verkuil, B., Brosschot, J. F., \& Thayer, J. F. (2007). Capturing worry in daily life: Are trait questionnaires sufficient? Behaviour Research and Therapy, 45, 1835-1844. 
Vosgerau, J., Wertenbroch, K., \& Carmon, Z. (2006). Indeterminacy and live television. Journal of Consumer Research, 32, 487-495.

Vrana, S. R., \& Lang, P. J. (1990). Fear imagery and the startle-probe reflex. Journal of Abnormal Psychology, 99, 189.

Wahl, K., Salkovskis, P. M., \& Cotter, I. (2008). 'I wash until it feels right': The phenomenology of stopping criteria in obsessive-compulsive washing. Journal of Anxiety Disorders, 22, 143-161.

Waters, A. M., \& Craske, M. G. (2005). Treatment for generalized anxiety disorder. In M. M. Antony, D. R. Ledley and R. G. Heimberg (Eds.), Improving outcomes and preventing relapse in cognitive-behavioral therapy (pp. 77-127). New York, NY: Guilford Press.

Watson, C., Burley, M. C., \& Purdon, C. (2010). Verbal repetition in the reappraisal of contamination-related thoughts. Behavioural and Cognitive Psychotherapy, 38, 337-353.

Wells, A. (2005). The Metacognitive Model of GAD: Assessment of meta-worry and relationship with DSM-IV generalized anxiety disorder. Cognitive Therapy and Research, 29, 107-121.

Wells, A., \& Carter, K. (2001). Further tests of a cognitive model of generalized anxiety disorder: Metacognitions and worry in GAD, panic disorder, social phobia, depression, and nonpatients. Behavior Therapy, 32, 85-102.

Wells, A., Welford, M., King, P., Papageorgiou, C., Wisely, J., \& Mendel, E. (2010). A pilot randomized trial of metacognitive therapy vs applied relaxation in the treatment of adults with generalized anxiety disorder. Behaviour Research and Therapy, 48, 429-434.

Wenzel, A. (2013). Strategic decision making in cognitive behavioral therapy. Washington, DC: American Psychological Association. 
Westra, H. A., Arkowitz, H., \& Dozois, D. J. (2009). Adding a motivational interviewing pretreatment to cognitive behavioral therapy for generalized anxiety disorder: A preliminary randomized controlled trial. Journal of Anxiety Disorders, 23, 1106-1117.

Westra, H. A., \& Dozois, D. J. (2006). Preparing clients for cognitive behavioral therapy: A randomized pilot study of motivational interviewing for anxiety. Cognitive Therapy and Research, 30, 481-498.

Westra, H. A., Dozois, D. J. A., \& Marcus, M. (2007). Expectancy, homework compliance, and initial change in cognitive-behavioral therapy for anxiety. Journal of Consulting and Clinical Psychology, 75, 363-373.

Whelton, W. J. (2004). Emotional processes in psychotherapy: Evidence across therapeutic modalities. Clinical Psychology \& Psychotherapy, 11, 58-71.

White, R. G., \& Gumley, A. (2010). Intolerance of uncertainty and distress associated with the experience of psychosis. Psychology and Psychotherapy: Theory, Research and Practice, 83, 317-324.

Widiger, T. A. (2011). The DSM-5 dimensional model of personality disorder: Rationale and empirical support. Journal of Personality Disorders, 25, 222-234.

Wilson, T. D., Centerbar, D. B., Kermer, D. A., \& Gilbert, D. T. (2005). The pleasures of uncertainty: prolonging positive moods in ways people do not anticipate. Journal of Personality and Social Psychology, 88, 5-21.

Xia, L., Kukar-Kinney, M., \& Monroe, K. B. (2010). Effects of consumers’ efforts on price and promotion fairness perceptions. Journal of Retailing, 86, 1-10. 
Yonkers, K. A., Dyck, I. R., Warshaw, M., \& Keller, M. B. (2000). Factors predicting the clinical course of generalised anxiety disorder. British Journal of Psychiatry, 176, 544549.

Yook, K., Kim, K., Suh, S. Y., \& Lee, K. S. (2010). Intolerance of uncertainty, worry, and rumination in major depressive disorder and generalized anxiety disorder. Journal of Anxiety Disorders, 24, 623-628.

Zinbarg, R. E., Craske, M. G., \& Barlow, D. H. (2006). Mastery of your anxiety and worry (therapist guide), $2^{\text {nd }}$ ed. New York, NY: Oxford University Press.

Zvolensky, M. J., Heffner, M., Eifert, G. H., Spira, A. P., \& Feldner, M. T. (2001). Incremental validity of perceived control dimensions in the differential prediction of interpretive biases for threat. Journal of Psychopathology and Behavioral Assessment, 23, 75-83. 